\title{
The role of intestinal and cardiovascular maturation in the adaptation to extrauterine life : from developmental biology to clinical intervention
}

Citation for published version (APA):

Rouwet, E. V. (2002). The role of intestinal and cardiovascular maturation in the adaptation to extrauterine life : from developmental biology to clinical intervention. [Doctoral Thesis, Maastricht University]. Universiteit Maastricht. https://doi.org/10.26481/dis.20020208er

Document status and date:

Published: 01/01/2002

DOI:

10.26481/dis.20020208er

Document Version:

Publisher's PDF, also known as Version of record

Please check the document version of this publication:

- A submitted manuscript is the version of the article upon submission and before peer-review. There can be important differences between the submitted version and the official published version of record.

People interested in the research are advised to contact the author for the final version of the publication, or visit the DOI to the publisher's website.

- The final author version and the galley proof are versions of the publication after peer review.

- The final published version features the final layout of the paper including the volume, issue and page numbers.

Link to publication

\footnotetext{
General rights rights.

- You may freely distribute the URL identifying the publication in the public portal. please follow below link for the End User Agreement:

www.umlib.nl/taverne-license

Take down policy

If you believe that this document breaches copyright please contact us at:

repository@maastrichtuniversity.nl

providing details and we will investigate your claim.
}

Copyright and moral rights for the publications made accessible in the public portal are retained by the authors and/or other copyright owners and it is a condition of accessing publications that users recognise and abide by the legal requirements associated with these

- Users may download and print one copy of any publication from the public portal for the purpose of private study or research.

- You may not further distribute the material or use it for any profit-making activity or commercial gain

If the publication is distributed under the terms of Article 25fa of the Dutch Copyright Act, indicated by the "Taverne" license above, 


\section{THE ROLE OF INTESTINAL AND CARDIOVASCULAR MATURATION IN THE ADAPTATION TO EXTRAUTERINE LIFE}


C Ellen Valérie Rouwet, Maastricht 2002 ISBN 90-9015566-X

Vormgeving en druk: Stereo+Grafia Maastricht 


\section{THE ROLE OF INTESTINAL AND CARDIOVASCULAR MATURATION IN THE ADAPTATION TO EXTRAUTERINE LIFE}

FROM DEVELOPMENTAL BIOLOGY TO CLINICAL INTERVENTION

\section{PROEFSCHRIFT}

ter verkrijging van de graad van doctor aan de Universiteit Maastricht, op gezag van de Rector Magnificus, Prof. dr. A.C. Nieuwenhuijzen Kruseman, volgens het besluit van het College van Decanen, in het openbaar te verdedigen op vrijdag 8 februari 2002 om 16.00 uur

door

Ellen Valérie Rouwet

geboren op 30 januari 1973 te Margraten 
Promotores

Prof. dr. G. Ramsay

Prof. dr. D.W. Slaaf

Copromotor

Dr. F.A.C. le Noble

Beoordelingscommissie

Prof. dr. P.B. Soeters (voorzitter)

Prof. dr. M.J.A.P. Daemen

Prof. dr. C. Ince (Universiteit van Amsterdam)

Prof. dr. ir. W.H.M. Saris

Prof. dr. G.J. Tangelder (Vrije Universiteit Amsterdam)

Financial support for the publication of this thesis by Nutricia Nederland B.V., Friesland Coberco Dairy Foods B.V., Bristol-Myers Squibb B.V., Byk Nederland B.V., Artesk Bureau voor Architectuur is gratefully acknowledged. 
Verlichting is het zegevieren van de mens over zijn zelfverkozen onmondigheid. Onmondigheid is het gebrek aan vermogen zijn eigen verstand te gebruiken zonder andermans leiding.

Deze onmondigheid is zelfverkozen als de oorzaak niet een gebrek aan verstand is, maar gebrek aan moed om het verstand te gebruiken.

Voor Verlichting is niets anders vereist dan vrijheid, die vrijheid welke inhoudt dat men in elk opzicht openbaarlijk van zijn verstand gebruik maakt.

Want het is de roeping van ieder mens om zelf te denken.

(Immanuel Kant, 1783) 
- 


\section{CONTENTS}

\section{Chapter 1}

General introduction

\section{Chapter 2}

Review of the literature

\section{Chapter 3}

Intestinal permeability and carrier-mediated monosaccharide absorption in preterm neonates during the early postnatal period

\section{Chapter 4}

Early minimal enteral feeding in very low birth weight neonates:

an effective strategy to improve mucosal functions of the immature gut

\section{Chapter 5}

Effect of repetitive asphyxia on leukocyte-vessel wall interactions in the developing intestine

\section{Chapter 6}

Development of vasomotor responses in foetal mesenteric arteries

\section{Chapter 7}

Chronic foetal hypoxia alters haemodynamic control

\section{Chapter 8}

General discussion

Summary

Samenvatting

Dankwoord 


\section{CHAPTER 1}

General Introduction 
Gastrointestinal complications ranging from mild feeding intolerance to fulminant necrotising enterocolitis are major causes of morbidity and mortality in preterm neonates, particularly in those of very low birth weight ( $<1500 \mathrm{~g}$ ). The incidence of necrotising enterocolitis is $1-3$ per 1000 live births, $1-8 \%$ of all admissions to the neonatal intensive care unit, and up to $10 \%$ in very low birth weight neonates. Necrotising enterocolitis is the single most common surgical emergency in preterm neonates. With an overall mortality rate of $29 \%$, it is expected that this disease will even surpass the respiratory distress syndrome as the principal cause of death in preterm neonates. In view of the growing number of preterm births and the improved survival rate of very low birth weight neonates, gastrointestinal complications will be of increasing concern in the management of these patients (1-13).

Necrotising enterocolitis (NEC) is a syndrome which is characterised by mucosal and transmural inflammation and necrosis of a single or multiple areas of the neonatal intestine, most commonly the terminal ileum and proximal colon (4). The clinical characteristics of NEC consist of abdominal distension, rectal bleeding, high gastric residuals, and vomiting. These abdominal signs are accompanied by systemic signs including lethargy, frequent periods of apnea, acidosis, bacteraemia, and shock (14). Although medical treatment is the first choice therapy, surgical resection of the necrotic part of the intestine or peritoneal drainage is indicated in case of clinical deterioration or intestinal perforation $(9,15)$. Infants which survive the acute stage of the disease are threatened by serious long-term sequelae, including gastrointestinal complications, such as intestinal strictures, fistulas, abscesses, and short bowel syndrome, as well as neurodevelopmental impairment (16-19).

Traditionally, three major inciting factors are implicated in the aetiology of NEC: feeding practices, intestinal bacterial flora, and intestinal hypoxia (Figure 1). Although many demographic reports have described these variables associated with NEC, none of these factors in isolation can adequately explain the pathogenesis, since no associated disease states are more common in affected neonates as compared with age-matched controls $(5-9,11,14,20-25)$. More than $90 \%$ of the neonates are born before term. Moreover, prematurity itself is the single consistently identified risk factor for this disease, the risk being highest in the youngest neonates (26). This supports the concept that NEC in preterm infants is a disease of multifactorial origin, being the final outcome of the interaction between an immature gastrointestinal tract and potentially injurious exogenous influences (1).

In case of preterm birth, the gastrointestinal tract is forced to adapt to the transition from the intrauterine to the extrauterine environment ahead of time. In neonates of very low birth weight, the intestine is exposed to the extrauterine environment at $0.6-0.8$ of total foetal gestation, i.e., as much as $15-8$ weeks too early. At that moment the basic morphologic development and the differentiation of the epithelial cells of the gut have been completed. However, the development of various functional aspects of the intestine occurs at a much slower pace (Chapter 2). Consequently, preterm birth exposes a functionally immature intestine to the stresses of extrauterine life, requiring precocious activation of its nutritive and barrier functions. It is hypothesised that failure of the immature intestine to adapt adequately to the transition from the intrauterine to the extrauterine environment predisposes the preterm neonate to intestinal complications. 


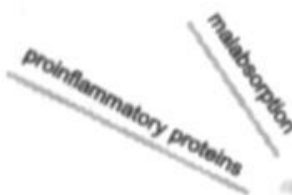

formula lipids

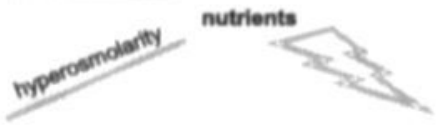

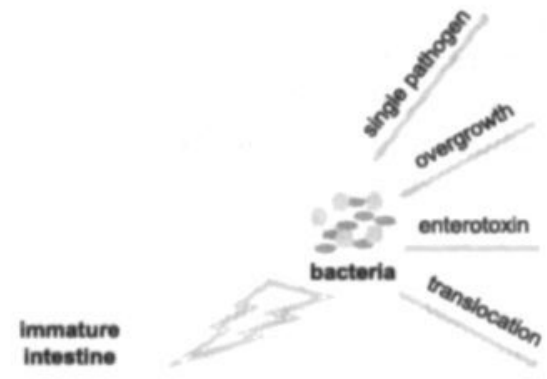

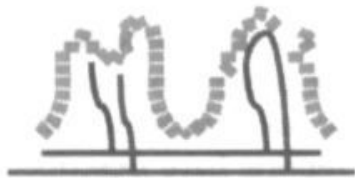

circulatory disturbances

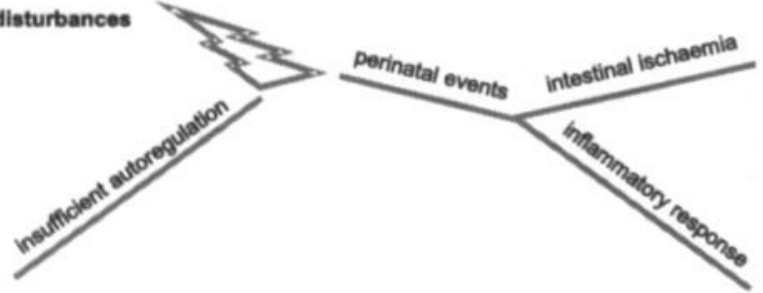

Figure 1. Aetiology of necrotising enterocolitis 


\section{Present thesis}

Insight into the origin of intestinal complications in preterm neonates requires a comprehensive understanding of the physiology and pathophysiology of the immature gastrointestinal tract. At present there is a paucity of information on this subject, since research in human foetuses and neonates is seriously confined by ethical and technical restrictions. Within the framework of the present thesis we sought to enhance the insight into the intrinsic development of the gut, as well as the interaction of the immature gut with its environment from both a gastroenterologic and a cardiovascular point of view, using an integrative clinical and basic experimental approach.

Chapter 2 summarises the current knowledge with regard to the structural and functional development of the human intestine, and presents an overview of the specific nutritional management of the preterm neonate. Growth and differentiation of the intestine, as well as the execution of its postnatal nutritive and barrier functions are ultimately dependent on the adequacy of intestinal blood supply. An overview of the complex regulation of the splanchnic circulation in the intestine is provided in the second part of Chapter 2.

Adaptation of the neonate to extrauterine life depends on the ability of the intestine to absorb nutrients from ingested food, while preventing host invasion by luminal microorganisms. The development of the absorptive capacity and the barrier function of the intestinal epithelium is investigated in preterm neonates born between 25 and 32 weeks gestation (Chapter 3 ). The consequences of early minimal enteral feeding as a nutritional intervention to enhance the maturation of these functions are studied in a randomised controlled trial (Chapter 4). Research concerning the splanchnic circulation in preterm neonates is limited. We therefore designed a novel experimental animal model for studying the microcirculation and resistance vasculature in the developing gut. The development of leukocytevessel wall interactions in the microcirculation of the immature intestinal wall is presented in Chapter 5 . The mechanisms which regulate blood flow to the immature intestine are studied in Chapters 6 and 7. The outcomes of the clinical and experimental studies are discussed in Chapter 8. Interpretation of these data in view of the pathophysiology of intestinal complications in preterm neonates conclude this thesis. 


\section{References}

1. Kliegman R.M., Walker W.A., and Yolken R.H. Necrotizing enterocolitis: research agenda for a disease of unknown etiology and pathogenesis. Pediatr Res 1993; 34: 701-708.

2. Grosfeld J.L., Cheu H., Schlatter M., West K.W., and Rescorla F.J. Changing trends in necrotizing enterocolitis. Ann Sung 1991; 214(3): 300-307.

3. Wilson R., Kanto W.P., MoCarthy B.J., Burton A., Lewin P., and Feldman R.A. Age at onset of necrotizing enterocolitis: an epidemiologic analysis. Pediatr Res 1982; 16: 82-84.

4. Albanese C.T. and Rowe M.I. Necrotizing enterocolitis. Semin Pediatr Surg 1995; 4(4): 200-206.

5. Kosloske A.M. Epidemiology of necrotizing enterocolitis. Acta Paediatr Suppl 1994; 396: 2-7.

6. Stoll B.J. Epidemiology of necrotizing enterocolitis. Clin Perinatol 1994; 21: 205-218.

7. Uauy R.D., Fanaroff A.A., Korones S.B., Phillips E.A., Phillips J.B., and Wright L.L. Necrotizing enterocolitis in very low birth weight infants: biodemographic and clinical correlates. J Pediatr 1991; 119; 630638.

8. Ryder R.W., Shelton J.D., and Guinan M.E. Necrotizing enterocolitis; a prospective multicenter investigation. Am JEpidemiol 1980; 112: 113-123.

9. Chandler J.C. and Hebra A. Necrotizing enterocolitis in infants with very low birth weight. Semin Pediatr Sung 2000; 9: 63-72.

10. Kramer M.S., Platt R., Yang H., Joseph K.S., Wen L.W., Morin L., and Usher R.H. Secular trends in preterm birth; a hospital-based cohort study. JAMA 1998; 280: 1849-1854.

11. Holman R.C., Stoll B.J., Clarke M.J., and Glass R.I. The epidemiology of necrotizing enterocolitis infant mortality in the United States. Am J Public Health 1997; 87: 2026-2031.

12. Dollfus C., Paletta M., Siegel B., and Cross A.W. Infant mortality. Pediatrics 1990; 86: 176-183.

13. Snyder C.L., Gittes G.K., Murphy J.P., Sharp R.J., Ashcraft K.W., and Amoury R.A. Survival after necrotizing enterocolitis in infants weighing less than $1000 \mathrm{~g}: 25$ years' experience at a single institution.

J Pediatr Surg 1997; 32: 434-437.

14. Rowe M.I., Reblock K.K., Kurkchubasche A.G., and Healey P.J. Necrotizing enterocolitis in the extremely low birth weight infant. J Pediatr Surg 1994; 29: 987-991.

15. Azarow K.S., Ein S.H., Shandling B., Wesson D., Superina R., and Filler R.M. Laparotomy or drain for perforated necrotizing enterocolitis: who gets what and why? Pediatr Surg Int 1997; 12: 137-139.

16. Horwitz J.R., Lally K.P., Cheu H.W., Vazquez W.D., Grosfeld J.L., and Ziegler M.M. Complications after surgical intervention for necrotizing enterocolitis: a multicenter review. J Pediatr Surg 1995; 30: 994 999.

17. Sonntag J., Grimmer I., Scholz T., Metze B., Wit J., and Obladen M. Growth and neurodevelopmental outcome of very low birthweight infants with necrotizing enterocolitis. Acta Paediatr 2000; 89: 528 532.

18. Vohr B.R., Wright L.L., Dusick A.M., Mele L., Verter J., Steichen J.J., Simon N.P., Wilson D.C., Broyles S., Bauer C.R., Delaney-Black V., Yolton K.A., Fleisher B.E., Papile L.A., and Kaplan M.D. Neurodevelopmental and functional outcomes of extremely low birth weight infants in the National Institute of Child Health and Human Development Neonatal Research Network, 1993-1994. Pediatrics 2000; 105: 1216-1226.

19. Ladd A.P., Rescorla F., West K.W., Scherer L.R., Engum S.A., and Grosfeld J.L. Long-term follow-up after bowel resection for necrotizing enterocolitis: factors affecting outcome. J Pediatr Surg 1998; 33: $967-972$.

20. Schober P.H. and Nassiri J. Risk factors and severity indices in necrotizing enterocolitis. Acta Paediatr Suppl 1994; 396: 4952. 
21. Lemelle JL, Schmitt M, de Miscault G., Vert P, and Hascoet J.M. Neonatal necrotizing enterocolitis: a retrospective and multicentric review of 331 cases. Acta Paediatr Suppl 1994; 396: 70-73.

22. Kanto W.P., wilson R, Breart G.L., Zierler S, Purohit D.M., Peckham G.J, and Ellison C. Perinatal events and necrotizing enterocolitis in premature infants. Am J Dis Child 1987; 141: 167-169.

23. Stoll B. J, Kanto W. ., Glass R. I, Nahmias A J, and Brann A. W. Epidemiology of necrotizing enterocolitis: a case control study. J Pudiatr 1980, $96: 447-451$.

24. De Curtis M., Paone C, Vetrano G., Romano G., Paludetto R., and Ciccimarra F. A case control study of necrotizing enterocolitis occurring over 8 years in a neonatal intensive care unit. Eur / Pudiatr 1987; 146. 398400 .

25. Kliegman R.M., Hack M, Jones P, and Fanaroff A.A. Epidemiologic study of necrotizing enterocolitis among low birth-weight infants; absence of identifiable risk factor. J Pudiatr 1982; 100:440-444.

26. Beeby PJ. and Jeffery H. Risk factors for necrotizing enterocolitis: the influence of gestational age. Anch Dis Child 1992; 67: 432-434. 


\title{
CHAPTER 2
}

\author{
Review of the Literature
}

\section{Part 1}

Intestinal development

Part 2

Splanchnic circulation

This at least I clearly know: that bread has different effects in the human body according as it is fine or coarse, made of wheat winnowed or unwinnowed, mixed with much or little water, kneed much or not at all, baked thoroughly or underbaked. and a thousand other differences besides. If one fails to consider these points or having considered does not understand them, how can he know anything about human disease? (Hippokrates, se eeuw v. Chr.) 


\section{INTESTINAL DEVELOPMENT}

The pathophysiology of intestinal complications in very low birth weight neonates appears to be closely related to the premature exposure of the intestinal tract to the extrauterine environment. Throughout foetal gestation, the bulk of nutrients is delivered to the foetus intravenously via the placental-umbilical route. The digestive tract is exposed to sterile amniotic fluid, the ingestion of which contributes to foetal gastrointestinal and somatic growth (1-3).

Birth induces an abrupt transition from the intrauterine to the extrauterine environment, which has important consequences for the demands placed on the intestinal tract. First, after delivery of the baby, the continuous intravenous nutrient supply via the placental-umbilical conduit ceases and the neonate becomes solely dependent on its gastrointestinal tract for the acquisition of sufficient nutrients from orally ingested milk feeding. Second, as soon as the neonate leaves the sterile intrauterine environment and passes through the maternal birth canal, the digestive tract comes into contact with a large amount of antigens each day. The intestinal lumen is being colonised with bacteria and other microorganisms, and is exposed to dietary antigens via the oral ingestion of food. The intestinal wall acts as a barrier which prevents that potentially pathogenic microorganisms, their endoand exotoxins, and dietary antigens invade the intestinal wall and gain access to the systemic circulation. Taken together, the switch from the intrauterine to the extrauterine environment requires the presence of the anatomical structures, as well as the activation of the nutritive and barrier functions of the intestine in order to support neonatal growth and survival during extrauterine life (Figure 1).

FOETUS

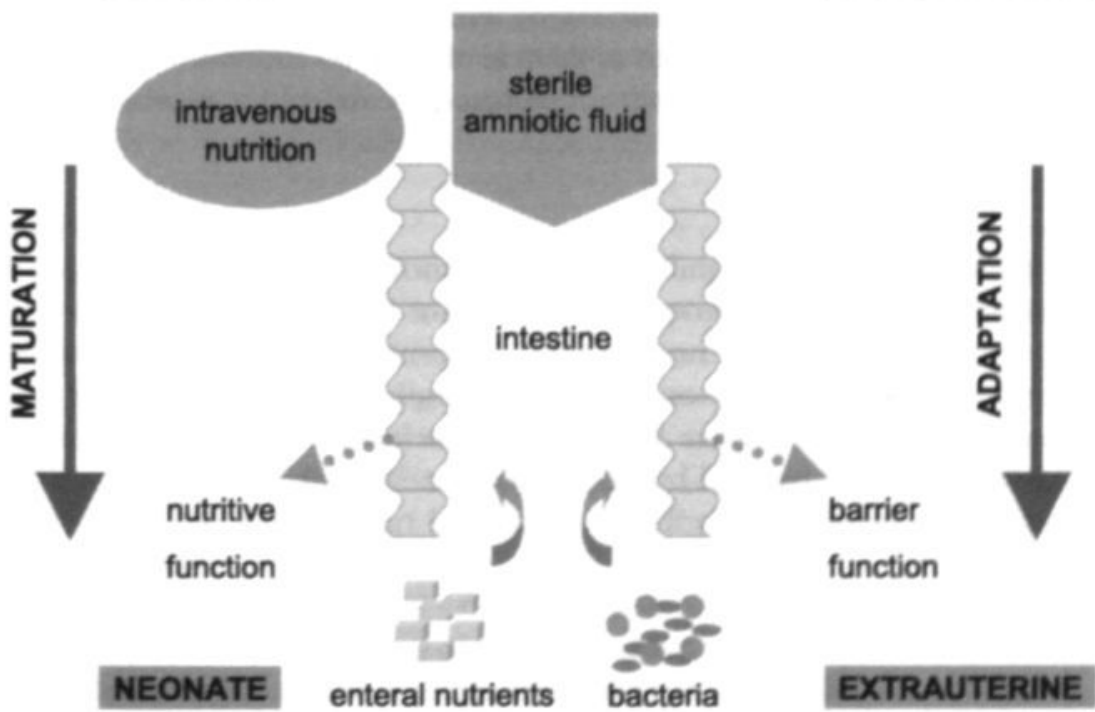

Figure 1. Transition from intrauterine to extrauterine environment 
Preterm birth is defined as birth prior to 37 weeks, i.e., prior to 0.9 of foetal gestation. The preterm neonates which are at highest risk for developing intestinal complications are born at less than 32 weeks, i.e., prior to 0.8 of foetal gestation. As a result of this early birth, the nutritive and barrier functions of the intestine are called upon ahead of time. The adaptation of the intestinal tract to extrauterine life depends on its structural and functional development at the time of birth.

The morphological development of the primitive gut in the human foetus starts during the fourth week of gestation. During subsequent weeks the basic structural components of the gut, consisting of mucosa, submucosa, and muscle layers, are formed. Between 10 and 12 weeks gestation, amplification of the epithelial surface commences by the formation of plicae circulares, crypts, villi, and microvilli. The structural development and the final anatomic position of the intestine are completed by 20 weeks gestation. The remaining period of gestation between 20 and 40 weeks is mainly dedicated to length growth and functional development of the intestine $(4,5)$.

The first part of this chapter provides an overview of the current knowledge on the functional development of the intestine in the human foetus. Insight into the time course of the maturation of the intestinal nutritive function is an essential prerequisite for adequate nutritional management of the preterm neonate, since the administration of oral feeding should obviously be adapted to the ability of the intestine to process and absorb nutrients from the intestinal lumen. In view of this, common feeding practices in the neonatal intensive care unit are discussed. 
At birth, the continuous intravenous nutrient supply from the mother via the placenta to the foetus ceases. The nutrient supply becomes fully dependent on intermittently administered oral milk or formula feeding. Consequently, peristaltic activity, regulated digestive enzyme production, and effective nutrient absorption must be present in the gastrointestinal tract at the time of birth. In this section an overview is given on the development of these nutritive functions of the human foetal intestine.

\section{Motor activity}

Oral feeding after birth requires the development of efficient coordination of sucking, swallowing, and intestinal motility. The first sucking movements in the human foetus already appear by 12 weeks, and foetal swallowing of amniotic fluid starts by 20 weeks gestation. The coordination of sucking and swallowing, how. ever, commences only by 34 weeks gestation (6). Intrauterine swallowing has several important functions. During the second half of gestation the foetus produces $500-1000 \mathrm{ml}$ of amniotic fluid daily, mainly in the form of urine and lung fluid. Since the foetus again ingests $300-1000 \mathrm{ml}$ of this, foetal swallowing is important in the regulation of amniotic fluid volume $(7,8)$. In addition, the absorption of nutrients from the amniotic fluid provides up to $10 \%$ of energy and nutrient requirements of the near term foetus and contributes to normal foetal growth (2,3,9-12).

Studies conducted in preterm infants have revealed the development of intestinal motor activity. Prior to 31 weeks gestation the small intestine displays only random contractions during fasting, with no motor response to food. Between 31 and 34 weeks gestation clustered contractions appear during fasting, and postprandial activity is induced. After 34 weeks gestation prolonged phasic activity with aboral propagation of intestinal contractions occurs during fasting, which is replaced by continuous contractile activity in response to enteral food. Migrating motor complexes, which are responsible for the propulsion of food, appear near term $(13,14)$.

\section{Digestion and absorption}

The acquisition of nutrients from the intestinal lumen requires that orally administered nutrients are digested into smaller components which are subsequently absorbed by the intestinal epithelium. This section summarises the current information on the appearance of digestive enzymes and the development of epithelial absorptive mechanisms for carbohydrates, lipids, and proteins in the human intestine. The major part of this information is derived from studies conducted in stillborn foetuses, and a minor part from studies performed in preterm neonates.

\section{Carbohydrates}

About $40-45 \%$ of the total energy content of human milk and formula is constituted by carbohydrates (15). Carbohydrates are mainly present in the form of lactose. The disaccharide lactose is digested to the monosaccharides glucose and galactose by the enzyme lactase, which is present at the epithelial brush border membrane in 
the small intestine. Lactase appears in the human foetal gut at 9 weeks gestation. The activity of lactase increases by 14 weeks gestation, remains at this level until 25 weeks, after which it rises again. By 34 weeks gestation lactase activity has increased to $30 \%$ of the term level, and by 38 weeks to $70 \%$. A further increase in lactase activity occurs during the first postnatal weeks (15-19). The activity of lactase is highest in the proximal and middle jejunum, and decreases along the distal end of the small intestine. Furthermore, lactase is predominantly located at the tips of the intestinal villi (20-22).

In view of the nutritional importance of lactose, attention is mainly focussed on the development of lactase in the neonatal gut. However, the appearance of other disaccharidases and polysaccharidases is relevant with respect to the preparation of the gut to the acquisition of carbohydrates from a more mixed diet during later postnatal life. Besides lactase, three other disaccharidases are present in the small intestinal brush border membrane: sucrase-isomaltase, maltase-glucoamylase, and trehalase. Sucrase-isomaltase and maltase-glucoamylase activities appear by 8 weeks gestation. The levels of these enzymes steadily increase until 14 weeks gestation, and rise more slowly thereafter. In contrast to lactase, the activity of these enzymes already reaches $70 \%$ of term levels by 34 weeks gestation. Trehalase activity is found in the foetal mucosa by 12 weeks, and reaches the adult level by 23 weeks gestation. Thus, the development of these enzymes precedes that of lactase. Furthermore, these enzymes express highest activity in the middle and lower region of the intestinal villi (20-22). Digestion of polysaccharides is mediated by the enzyme amylase present in pancreatic fluid and saliva. Secretion of amylase by the foetal pancreas is low, even at birth. In contrast, salivary amylase already appears in the human foetus at 16 weeks gestation, and is therefore the most important enzyme in polysaccharide digestion in the neonate $(23,24)$.

The digestion of carbohydrates generates the monosaccharides glucose, galactose, and fructose, which are absorbed by the enterocytes in the small intestine. Glucose and galactose are absorbed by active carrier-mediated transport. Transport of these monosaccharides across the brush border membrane is mediated by the $\mathrm{Na}^{+} /$glucose carrier protein SGLT1. This transport is an energy-dependent process, since it is driven by a sodium gradient across the brush border membrane, which is maintained by the $\mathrm{Na}^{+} / \mathrm{K}^{+}$-ATPase at the basolateral membrane. Efflux of glucose and galactose at the basolateral membrane of the enterocytes into the blood occurs by facilitated diffusion, which is mediated by the transporter protein GLUT2. In contrast to glucose and galactose, fructose is absorbed by carrier-mediated transport along its concentration gradient, which is not an energy-dependent process. Transport of this monosaccharide at the brush border membrane is mediated by the carrier protein GLUT5, and at the basolateral membrane by GLUT2. These carrier proteins are expressed in fully differentiated enterocytes lining the upper third of small intestinal villi $(25,26)$.

Active glucose transport is present in the human jejunum by 10 weeks gestation, and increases between 10 and 19 weeks $(27,28)$. Glucose transport is more pronounced in the jejunum as compared with the ileum of the human foetus, accounting for the proximal-to-distal transport gradient which is also present in the mature intestine $(29,30)$. Information with regard to the further maturation of sugar 
transport during the second half of foetal development is lacking. Consequently, it is not known to what extent the intestine of the preterm neonate is adapted to absorb sugars, and when glucose absorptive capacity reaches the level of the term neonate (31).

\section{Lipids}

Fat constitutes about $50 \%$ of the total energy content of milk and formula. Lipid digestion depends on the presence of bile salts and lipases in the intestinal lumen. Bile salts act as detergents in the jejunum, promoting the subsequent digestion of dietary fats by lipases. Bile salts are synthesised and secreted by the liver, and enter the duodenum via the biliary tract in the form of bile. Most of the bile salts are reabsorbed in the terminal ileum, and return to the liver via the portal venous blood to be secreted again into the bile. Although hepatic synthesis and secretion, intestinal reabsorption, and hepatic uptake of bile salts all appear during gestation, these functions are still immature at birth, resulting in low concentrations of intraluminal bile salts in neonates (26).

Lipids are digested by lipases secreted by the pancreas and stomach. Pancreatic lipase activity is first detected in the human foetus at 21 weeks gestation, and is still very low at birth. In contrast, gastric lipase is already secreted by 10 weeks gestation, and further increases with subsequent foetal development. Therefore, gastric lipase is the most important mediator of lipid digestion in the neonate. The secretion of pancreatic lipase increases toward adult levels within the first 6 months after birth, becoming the major mediator of fat digestion during later postnatal life $(23,32)$.

The free fatty acids and monoglycerides generated by the hydrolysis of fat in the intestinal lumen are subsequently absorbed by the enterocytes. The diffusion of fatty acids and monoglycerides through the cell membrane, their intracellular binding to fatty acid-binding protein, and the formation of chylomicrons, is initiated between 14 and 20 weeks gestation and increases slightly after birth (33). Further insight into the development of the various stages of lipid absorption in the human foetus remains to be established. The retention of fat from food increases after birth and reaches $90 \%$ of the adult level at about one month of age (34).

\section{Proteins}

Proteins contribute 5.5-8\% to the total energy content of human milk and formula, and are necessary to support neonatal growth. After oral ingestion, proteins are hydrolised into smaller peptides and amino acids by proteolytic enzymes, which are subsequently absorbed by small intestinal enterocytes. Digestion of proteins starts in the upper gastrointestinal tract by proteases secreted in the gastric and pancreatic juices. Proteolysis in the stomach depends on two factors: the secretion of pepsin by gastric chief cells, and the production of gastric acid by parietal cells. Pepsin is already present by 16 weeks gestation (32). However, gastric acid output in neonates is still low, and starts to increase in the course of the first year of life (35). As a result, gastric proteolytic activity in the newborn infant is only $2 \%$ of the adult level (23). 
Pancreatic secretion of the proteases trypsin and chymotrypsin starts at 20 weeks gestation and rapidly increases after birth in both preterm and full-term neonates $(36,37)$. The activation of these pancreatic proteases is critically dependent on enterokinase, which is present in the brush border membrane of the proximal small intestine. This enzyme is first detected at 24 weeks, and increases during gestation. At term birth the enterokinase activity is still only $25 \%$ of that in older infants (19).

Further digestion of proteins is mediated by various peptidases present in the brush border membrane of the small intestine. These peptidases appear by 8 weeks gestation, and their activity rises between the early foetal period and childhood (38). Taken together, the proteolytic activity at the time of birth is low at all levels of the digestive tract, resulting in a limited capacity to digest proteins.

The small peptides and amino acids which are generated by the digestion of proteins are subsequently absorbed by the enterocytes in the small intestine. After absorption, dipeptides and tripeptides are further reduced to amino acids by soluble peptidases in the cytoplasm of the enterocytes. Data on the development of peptide and amino acid transport, as well as of intracellular peptidases in the human foetus are limited. Active transport of amino acids across the enterocyte brush border membrane is first detected between 15 and 18 weeks gestation, as is the presence of soluble peptidases $(39,40)$. A special form of protein absorption is the intact uptake of some biologically active proteins via endocytosis (41). This macromolecular uptake of growth factors, hormones, and immunoglobulins from amniotic fluid and maternal milk may contribute to the development of the digestive tract $(42-47)$.

It is clear from the previous paragraphs that the maturation of intestinal motor activity, digestive enzyme secretion, and absorptive capacity mainly occurs in the course of the third trimester of foetal gestation. Consequently, when an infant is born before term the development of various components of the intestinal nutritive function has not proceeded to the extent that the neonate can fully rely upon the gastrointestinal tract to fulfil its nutritional demands. This obviously has important implications for the nutritional management of the preterm neonate. 


\section{DEVELOPMENT OF INTESTINAL BARRIER FUNCTION}

The gastrointestinal tract of the foetus is sterile. However, as soon as the foetus leaves the sterile surroundings of the maternal uterus and is exposed to the extrauterine environment, microorganisms enter the intestinal lumen. A major function of the gut is to protect the neonate from invasion by these potentially pathogenic microorganisms, as well as by their endo- and exotoxins, which is referred to as the intestinal barrier function.

\section{Bacterial colonisation}

The first microorganisms acquired by the neonate are those which are transferred from the maternal cervix, vagina, and anus during vaginal delivery, or from the environment in the case of caesarean section. After birth, the intestinal tract is exposed to cutaneous, oral, and environmental microorganisms from the mother or nursing staff during neonatal care, as well as to those that enter with food. Although the neonatal gastrointestinal tract is rapidly exposed to a variety of bacterial and non-bacterial species, not all these will subsequently colonise the gut. Specific bacteria become established in particular hosts during various phases of development, a process referred to as bacterial succession.

A schematic overview of the bacterial colonisation pattern in the neonatal gut is shown in Figure 2. In full-term infants, the intestinal colonisation pattern is influenced by the type of enteral feeding. Neonates which are fed human milk are predominantly colonised by bifidobacteria and lactobacilli, whereas the intestine of formula-fed neonates contains high numbers of bacteroides and clostridia. These differences disappear after the introduction of solid food in the diet, when the intestinal microflora shifts towards a mature pattern. Bacterial succession in the preterm neonate is different from that in the term neonate. This difference may be related to the relatively aseptic environment of the neonatal intensive care unit, intravenous antibiotic therapy, and delay in the initiation of enteral feeding (48-50). In contrast to full-term infants, the colonisation pattern in preterm neonates is not affected by the type of enteral feeding, nor is it influenced by gestational age, birthweight, mode of delivery, maternal antibiotic or steroid treatment, or duration of rupture of the membranes. The intestine of the preterm neonate contains relatively few species, low numbers of lactobacilli and bifidobacteria, and high numbers of enterococci and staphylococci, including $S$. epidermidis and $S$. haemolyticus. The numbers of lactobacilli and bifidobacteria, as well as the total bacterial counts increase with postnatal age. The intestinal microflora of the preterm neonate starts to resemble that of the full-term neonate by the end of the first month after birth (48,51-59). 


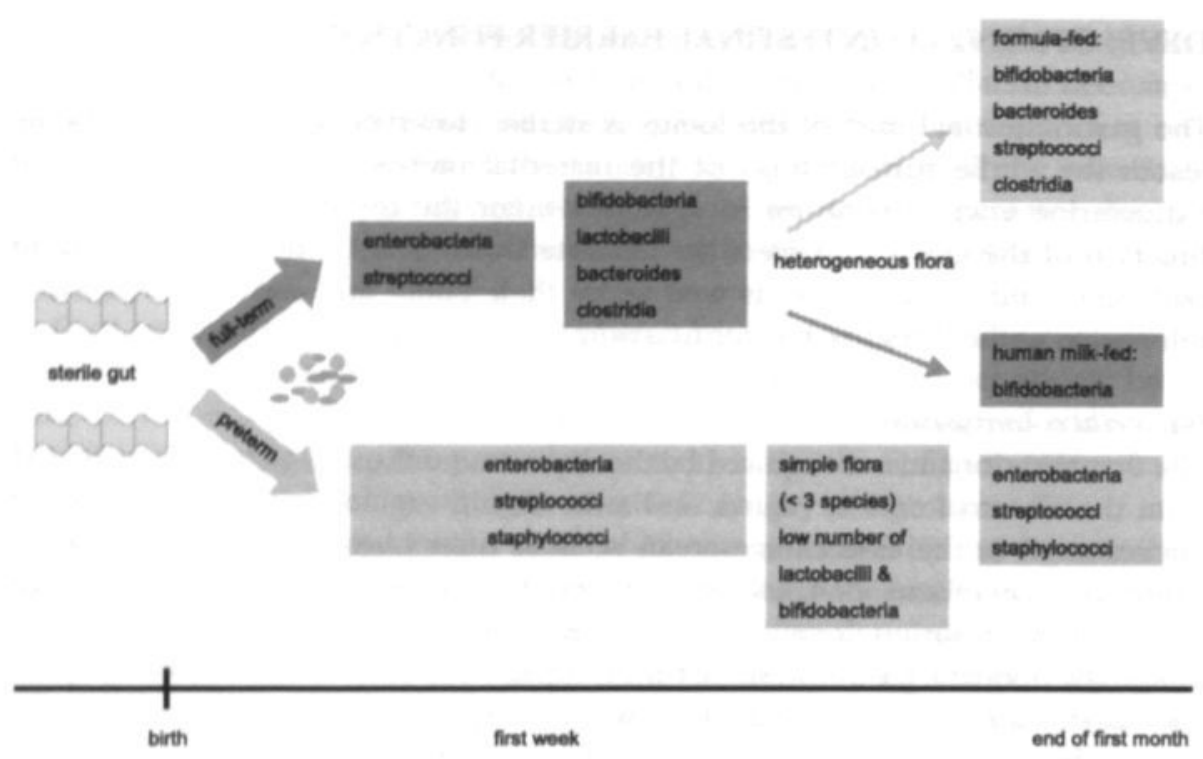

Figure 2. Bacterial colonisation of the neonatal intestine

\section{Intestinal barrier function}

In order to prevent luminal microorganisms and their products from invading the intestinal wall and systemic circulation, the gut is equipped with an elaborate system of non-immunologic and immunologic defence mechanisms, collectively referred to as the intestinal barrier function. In addition, the passage of dietary antigens from the gut lumen to underlying immune cells is limited by proper mucosal barrier functioning, which is essential to controlling antigenic responses and ensuring systemic tolerance.

Structural and functional components of the intestinal barrier include:

- gastric acid and digestive enzyme secretion

- peristalsis

- mucus layer

- intestinal epithelium

- nonspecific host defence (including leukocytes and secretory antibacterial peptides)

- immune system:

gut associated lymphoid tissue (Peyer's patches, lamina propria lymphocytes, intraepithelial lymphocytes)

secretory immunoglobulins (sIgA, sIgM)

Although immaturity of local defences has been proposed to be involved in the pathogenesis of NEC, information on the development of the various aforementioned components of the gut barrier in humans is scarce $(42,60-65)$.

An important part of the first-line defence to the penetration of noxious substances is constituted by the intestinal epithelial lining, acting as a physical barrier 
and actively participating in mucosal immune responses (66). The physical barrier function of the intestinal epithelium is dependent on the close interaction of adjacent epithelial cells. To this end, epithelial cells are joined by tight junctions, which form a circumferential seal at the apical pole of adjacent cells, and delineate the distinct apical and basolateral regions of the enterocyte cell membrane. The tight junction complex consists of various proteins, such as zonula occludens protein-1/2/3, occludin, and claudins. The tight junctions do not form a rigid barrier in the intercellular space, but are influenced by intracellular and external factors, including cytokines, growth factors, synthetic peptides, and toxins. Attenuation of the tight junctions is associated with increased intestinal paracellular permeability, resulting in the penetration of luminal antigens (67-72).

\section{Role of bacterial colonisation in intestinal complications}

A role for intestinal microorganisms in the aetiology of NEC is suggested by several clinical and epidemiologic observations. NEC does not occur in utero, usually occurs at a time when the intestine is fully colonised, and is accompanied by bacteraemia in $30 \%$ of cases. Moreover, cases of NEC are often clustered in time and place, are associated with an increased frequency of gastrointestinal symptoms among the nursery staff, and may be interrupted by infection control measures (73-78).

The damage in NEC may be due to direct infection of the intestinal tissue by a single pathogen. Enteropathogenic organisms which have been implicated in cases of NEC include Salmonella, rotavirus, coronavirus, and enterovirus. Furthermore, the similarities between the pathologic findings in NEC and in enteric infections caused by Clostridial species, including pigbel and pseudomembranous colitis, have suggested that NEC is elicited by a bacterial enterotoxin, including toxins released by Clostridium difficile and Clostridium perfringens type $\mathrm{C}$, or delta-toxin produced by coagulase-negative staphylococci $(79,80)$.

Although these observations indicate the involvement of intestinal microorganisms in the pathogenesis of NEC, the absence of a single common pathogen suggests that NEC is not a primary infectious disease, but rather results from secondary invasion of the intestine after mucosal disruption. The aberrant colonisation pattern in preterm neonates combined with an immature gut barrier may allow overgrowth and subsequent translocation by strains of common enteric microorganisms, including Escherichia, Enterobacter, Klebsiella, Clostridium, Pseudomonas, and Staphylococcus species $(51,58,81-83)$. This hypothesis is supported by the demonstration of NEC-like lesions in gnotobiotic animal models inoculated with a single nonpathogenic Clostridium strain $(84,85)$. 
In the preterm infant, many aspects of the motor activity, digestive enzyme secretion, and nutrient absorption in the gastrointestinal tract are still immature. In view of this immaturity the normal principles of enteral feeding for healthy full-term infants cannot be applied to preterm neonates. With the emergence of necrotising enterocolitis as one of the most common causes of early postnatal mortality in preterm neonates, dietary management of these neonates has become a main focus of attention in neonatal intensive care.

According to the American Academy of Pediatrics, the goal of nutritional management of the preterm neonate is to achieve a postnatal growth rate that approximates the intrauterine growth rate of the normal foetus at the same postconceptional age, without imposing stress on the infant's immature intestinal, metabolic, and excretory functions (86). In order to accomplish this goal, it is common practice in most neonatal intensive care units to initiate intravenous nutrition shortly after birth, and to replace this with enteral nutrition as the neonate matures and its medical condition stabilises. The shift from complete parenteral to complete enteral nutrition (total caloric intake $>100 \mathrm{kcal} / \mathrm{kg} /$ day) is a gradual process that may take several weeks, especially in very low birth weight neonates (87-94).

\section{Parenteral nutrition}

Intravenous nutrition is the mainstay of the nutritional management during the early postnatal period in preterm neonates, especially those under 32 weeks gestation. Intravenous nutrition consists directly after birth of dextrose solutions, in order to maintain glucose homeostasis as well as fluid and electrolyte balance. Since nutrient stores are only limited, nutritional support by means of total parenteral nutrition is usually initiated at the first or second day of life, and consists of amino acids, glucose, and lipid emulsion with added electrolytes and vitamins. Analogous to the intrauterine situation, these nutrients are directly infused into the circulation. The composition and volume of the infusate are adjusted in response to clinical and chemical monitoring of the neonate. In many neonatal centers preterm neonates are exclusively parenterally fed during the first one or two weeks after birth in an attempt to spare the immature gastrointestinal tract, and prevent the development of enteral feeding-related intestinal complications $(91,93,95)$.

Frequent complications of total parenteral nutritition are related to the insertion of intravenous catheters, including thrombophlebitis and sepsis. Furthermore, total parenteral nutrition is associated with metabolic complications, such as cholestatic jaundice, osteopenia, and disturbances in plasma levels of glucose, amino acids, fatty acids, and electrolytes (91).

\section{Enteral nutrition}

After a period of exclusive parenteral feeding, enteral nutrition is introduced and advanced according to the clinical condition of the neonate until all nutrients can be delivered via the gastrointestinal route. The absence of coordinated sucking and swallowing (8) requires that enteral feeding is administered directly into the stomach via a nasogastric tube in neonates born prior to 34 weeks gestation. Even 
then, the further propulsion of food from the stomach to the large intestine is slow, because migrating motor complexes during fasting and intestinal contractions in response to luminal food are largely absent. Because of slow intestinal transit, enteral feeding is initiated cautiously in these neonates $(96,97)$. The optimal timing, volume, rate of increment, type, and mode of enteral nutrition for the preterm neonate are still subject of debate (98).

\section{Type of enteral feeding}

There is universal agreement that human milk is the first choice enteral feeding for the term infant. No such standard has been set for the precise nutritional needs of the preterm neonate $(98,99)$. Feeding human milk during the early postnatal period in preterm infants is associated with improvements in gastrointestinal functioning, including nutrient digestion and absorption, gastrointestinal motility, neonatal defence against infection, and reduced incidence of necrotising enterocolitis (99-102). Additional beneficial effects of human milk include enhanced brain stem maturation, better long-term neurodevelopmental outcome and lower blood pressure (103-105). The beneficial effects of human milk may be related to the presence of a variety of bioactive factors, including digestive enzymes, growth factors, hormones, and neuropeptides, as well as antimicrobial, immunomodulatory, and antiinflammatory agents $(64,106-110)$.

Nonetheless, in addition to variability in composition and availability, as well as losses during storage procedures, human milk does not entirely meet the estimated nutritional requirements of the preterm neonate (109). Human milk beyond the first few weeks after birth has a low energy content and provides insufficient quantities of protein, calcium, phosphorus, and sodium. Unfortified human milk feeding to preterm neonates may fail to support intrauterine growth rates, and may result in poor bone mineralisation and hyponatraemia (110). Supplementation of expressed breast milk with commercial fortifiers to augment its energy and nutritional contents improves nutritional status, weight gain, and growth in preterm infants $(94,111,112)$.

For preterm neonates, the most appropriate substitute for human milk is preterm formula, which is designed to meet the calculated nutritional needs of these patients (92). As compared with regular formula, preterm formula contains increased amounts of energy, whey protein, medium chain triglycerides, minerals, vitamins, and trace elements, as well as a reduced lactose load. These supplemented nutrients may account for the better neurodevelopmental outcome in neonates fed preterm formula during the early postnatal period as compared with those fed standard term formula (113). Furthermore, preterm formula feeding has been associated with even higher rates of weight and length gain as compared with fortified human milk feeding (102).

\section{Early minimal enteral feeding}

The common feeding policy in preterm neonates discussed in the previous sections has been designed to follow the natural course of events which would have taken place during this period if the neonate had not been born prematurely. Intravenous nutrition is the sole mode of nutrient supply during the first few weeks 
after birth, as would be the case at this time during intrauterine life.

The nutritional management of preterm neonates is adapted to the maturational process of the digestive tract. However, one of the major differences between intravenous nutrient supply to the foetus and parenteral nutrition of the preterm infant is the absence of amniotic fluid in the lumen of the digestive tract in the latter. During intrauterine development, the foetal gut is continuously exposed to amniotic fluid. Experimental studies suggest that the ingestion of amniotic fluid by the foetus is necessary for normal development of the gastrointestinal tract $(1,114-117)$. This may be related to the presence of growth factors and hormones in the amniotic fluid, such as epidermal growth factor, insulin and glucocorticoids (33,118-120). Therefore, the absence of amniotic fluid in the gut lumen may interfere with the development of the gastrointestinal tract in the preterm neonate.

In addition, in case of exclusive parenteral feeding, the gut of the preterm neonate is deprived of enteral nutrients. It has been shown both in neonatal animals and in adult patients that enteral starvation is associated with inhibition of intestinal growth, mucosal atrophy, a reduction of lactase and sucrase levels, decreased absorption, and increased bacterial translocation (121-123). Hence, withholding enteral feeding from preterm neonates, which was originally intended to protect the immature gut, may actually be counterproductive to aiding the normal maturation of the intestinal tract that started in utero with the ingestion of amniotic fluid.

In an attempt to avoid these negative effects of enteral starvation without the risks of full-volume enteral nutrition, the concept of early minimal enteral feeding in preterm neonates has evolved. Early minimal enteral feeding is defined as the administration of nutritionally insignificant quantities of enteral feeding (12-24 $\mathrm{ml} / \mathrm{kg} /$ day) soon after birth, while delivering the majority of nutrients parenterally. Various terminologies are used for these small feedings, including minimal enteral nutrition, gut priming, trophic feeding, and hypocaloric feeding (124). The effects of early enteral feeding on short-term clinical outcome have been the subject of investigation in several trials (Table 1). The mechanisms underlying the reported beneficial influences of early enteral feeding on gastrointestinal functioning have not been elucidated yet. Postulated mechanisms include augmented release of intestinal hormones (125), reduction of intestinal permeability (126), and stimulation of lactase activity (127) and gut motility (128-130). 
Increased weight gain and head growth

Less metabolic bone disease

Reduction of physiologic and cholestatic jaundice

Reduction in the days to full enteral feeding*

Reduction in the total number of days that enteral feeding was withheld*

Reduction in total hospital stay*

Less episodes of sepsis

Fewer days of supplemental axygen

No increase in the incidence of necrotising enterocolitis ${ }^{*}$

"consistently demonstrated in a systematic meta-analysis of these studies (135)

\section{Role of enteral feeding in intestinal complications}

A relationship between enteral feeding and necrotising enterocolitis is suggested by the epidemiologic observation that the majority of affected neonates has received enteral feeding prior to the onset of clinical symptoms (77). However, the disease also occurs in neonates which have never been fed, and delayed introduction of oral feeding does not lower the incidence of NEC $(136,137)$.

Several mechanisms have been postulated to explain this relationship. Enteral administration of hyperosmolar feeding or hypertonic drug solutions may cause direct mucosal damage. Moreover, specific components of human milk or formula, such as casein, whey protein, and lipids, may contribute to the induction of intestinal tissue injury either directly or via the release of inflammatory mediators (138-142). It has also been postulated that the combination of enteral feeding and bacteria in the gut lumen leads to mucosal damage, as a result of bacterial fermentation of unabsorbed carbohydrates and/or promotion of bacterial proliferation $(81,143)$. 


\section{AIMS OF THE CLINICAL STUDIES}

Adequate adaptation of the preterm neonate to the stresses of extrauterine life is in part dependent on the ability of the intestine to absorb nutrients from ingested food while preventing invasion by potentially harmful agents from the intestinal lumen. To date, information on human functional intestinal development and the regulation thereof is scarce and are largely deduced from in vitro studies in intestinal tissue of stillborn human foetuses. The first aim of the clinical studies presented in this thesis was to elucidate the early postnatal development of the nutritive and barrier functions of the intestinal epithelium in preterm neonates (Chapter $3)$.

Preterm birth requires a precocious activation of intestinal nutritive and barrier functions. Experimental evidence indicates that nutrients present in the intestinal lumen are important regulators of enterocyte function. The second aim of the clinical studies was to assess whether the administration of small amounts of enteral feeding soon after birth is a useful nutritional intervention to enhance the maturation of these intestinal epithelial functions in preterm neonates. In a randomised controlled trial we investigated the effect of early minimal enteral feeding on the development of intestinal absorptive capacity and epithelial integrity in neonates born between 25 and 32 weeks gestation (Chapter 4 ). 


\section{SPLANCHNIC CIRCULATION}

The structural and functional differentiation of the gut, as well as the maintenance of its motor, digestive, absorptive and barrier activities are dependent on adequate intestinal blood supply. In order to deliver oxygen and substrates for the energydependent processes in the intestinal tissue, and to remove absorbed nutrients, electrolytes, fluids, and metabolic waste products, an extensive vascular network is established in the intestinal tract. The perfusion of the splanchnic circulation is controlled by systemic as well as local factors. These factors have been well characterised in the adult gut, and most of this information is derived from experimental animal studies. However, information regarding the control of the splanchnic circulation in the foetal and neonatal gut is limited.

Adequate adaptation of the preterm gut to extrauterine life is obviously dependent on the capacity of the splanchnic circulation to support its metabolism. Insight into the maturation of the regulation of intestinal blood supply during foetal development is prerequisite to understanding the physiology of the intestinal circulation in preterm neonates. This section presents a general overview of the regulation of blood supply in the adult and neonatal gut.

\section{Vascular anatomy of the human intestine}

The arterial supply to the small intestine is derived from branches of the superior mesenteric artery, which pass between the two layers of the mesentery toward the mesenteric margin of the bowel. After coursing over the serosal surface of the intestine, the mesenteric arteries give rise to smaller branches which penetrate the longitudinal and circular muscle layers of the gut to enter the submucosal arterial plexus. From there, blood is distributed to the muscle layers, submucosa, and mucosa. Mucosal arterioles originate from the submucosal arterial plexus, ascend each villus, and branch into capillaries in a fountain pattern at the villus tip. The villus shaft and intestinal crypts are supplied by a separate pericryptal capillary network, arising from the same submucosal arteriole. The subepithelial capillary plexus drains into a single venule that descends the villus to join the submucosal venous plexus. From the submucosal veins further venous drainage of the bowel parallels its arterial supply, and ends in the portal vein (144).

\section{Regulation of intestinal blood flow}

Intestinal blood flow, blood oxygen content, and tissue oxygen extraction determine the oxygen supply at the tissue level. Blood flow through the intestinal circulation is dependent on the arteriovenous blood pressure gradient, and on resistance to flow of the intestinal vascular tree. Resistance to flow, in turn, is determined by vascular topology and diameter. Arterial diameter depends on arterial smooth muscle tone, the contractile state of the smooth muscle cells, which is controlled by both extrinsic (systemic) and intrinsic (local) mechanisms. An overview of the complex regulation of the intestinal blood flow is shown in Figure 3. 


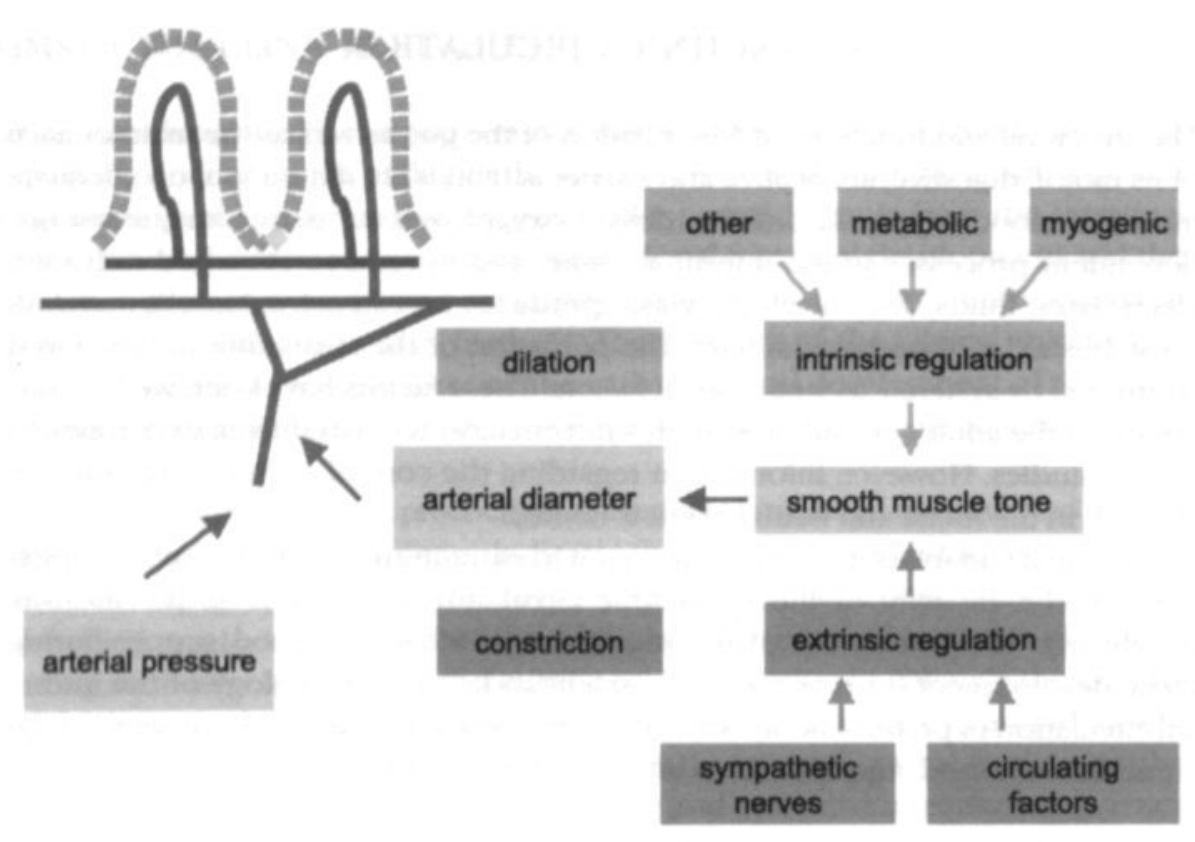

Figure 3. Regulation of intestinal blood flow

\section{Extrinsic regulation}

Vasoconstrictor control of the intestinal circulation is designed to restore cardiovascular homeostasis under conditions of haemodynamic instability, such as hypotension or hypoxia. It serves to protect the whole organism, even at the expense of the intestinal perfusion. Regulation of the splanchnic perfusion as one of the major peripheral vascular beds contributes to the control of the systemic blood pressure. To this end, the intestinal arterial diameter is regulated by a number of neural and humoral mechanisms which originate extrinsic to the gut.

Neural control is exerted by postganglionic sympathetic nerve fibers which innervate the mesenteric vessels and the submucosal vascular plexus of the gut, and which predominantly release norepinephrine at the terminal nerve endings. These sympathetic nerve fibers arise from neurons in the intermediolateral area of the lumbosacral segments of the spinal cord. The preganglionic nerve fibers join to form the greater, lesser, and least splanchnic nerves as well as the lumbar splanchnic nerves, which supply the coeliac, superior mesenteric, and inferior mesenteric ganglia. Neurons in these prevertebral ganglia give rise to postganglionic axons which form the perivascular sympathetic nerves. The sympathetic neurons in the spinal cord are controlled by descending nerve pathways from the vasomotor centre in the brain stem, which in turn receives afferent input from the baroreceptors and chemoreceptors (145).

Humoral extrinsic control of the intestinal circulation is mainly exerted by circulating epinephrine and norepinephrine released from the adrenal medulla. Catecholamine release from the adrenal gland is controlled by preganglionic sym- 
pathetic nerves, and is also directly influenced by the arterial oxygen concentration (146-148). Furthermore, other systemic vasoactive substances, such as vasopressin and angiotensin II contribute to the vasoconstrictor control of the intestinal circulation (149).

Information regarding onset and maturation of the extrinsic regulation of intestinal perfusion has been deducted from data on the cardiovascular response to acute hypoxia in foetal animals at different stages of development. In foetal sheep at 0.9 gestation, an acute reduction in blood oxygen content was associated with a decrease in intestinal blood flow $(150,151)$. This decrease in blood flow could be prevented by the systemic administration of an $\alpha$-adrenergic antagonist, as well as by chemical sympathectomy, indicating the involvement of $\alpha$-adrenoceptors and sympathetic nerves in the control of intestinal blood flow at this stage $(152,153)$. Since no reduction in intestinal blood flow was observed in foetuses of younger gestation, it was suggested that extrinsic control of intestinal arterial diameter commenced at the end of foetal development (154). Comparable responses were found in the developing chick foetus $(155,156)$.

In these studies, changes in intestinal blood flow were determined, by means of a microsphere technique or flow probe, not alterations in arterial diameter. Although both methods can adequately measure absolute or relative changes in total organ blood flow, the mechanisms underlying these changes cannot be identified by either of these techniques. As was indicated in a previous paragraph, blood flow is determined by a large number of local and systemic variables. A reduction in intestinal blood flow may be secondary to active constriction of the mesenteric resistance arteries (or the more distal intestinal vasculature), shunting of blood towards other (dilated) vascular beds, or a reduction in cardiac output. Therefore, although the aforementioned observational studies provide a first indication of the regulation of intestinal perfusion, they do not provide insight into the site and mechanisms of extrinsic control of intestinal vascular tone, as well as the developmental changes herein.

\section{Intrinsic regulation}

Intrinsic regulation of the blood flow to the intestinal tissue serves to adapt oxygen delivery to metabolic demand of the intestinal tissue, and to maintain intestinal perfusion under conditions of altered systemic haemodynamics. Two major mechanisms are involved in the intrinsic control of diameters of intestinal resistance arteries and arterioles in the gut wall, i.e., metabolic and myogenic feedback.

Metabolic feedback regulates intestinal blood flow proportionally to tissue oxidative demand. Tissue $\mathrm{PO}_{2}$ is the principal variable controlled by this mechanism. Increased metabolism is associated with tissue hypoxia and accumulation of vasodilator metabolites, such as adenosine, $\mathrm{CO}_{2}, \mathrm{H}^{+}$, lactate, and histamine (157). Dilation of precapillary arterioles by metabolic feedback increases blood flow and oxygen diffusion area in the intestinal microcirculation, thus enhancing oxygen extraction in the gut $(158,159)$. In the adult, arterioles are more sensitive to metabolic feedback than resistance arteries, suggesting that metabolic regulation of resistance arterial diameter comes into play only when the capacity of exchange vessels to maintain tissue oxygenation is insufficient (160). 
Myogenic feedback modulates arterial smooth muscle tone in response to changes in perfusion pressure, and is based on the capacity of vascular smooth muscle cells to contract in response to a stretch stimulus. Circumferential vascular wall tension is the principal variable controlled by this mechanism. A reduction in perfusion pressure is associated with an increase in intestinal arterial diameter, while the opposite occurs in response to a rise in arterial pressure (161-163).

In addition, local vascular control is exerted by a large number of other vasoactive substances which are released within the intestinal parenchyma, including gastrointestinal hormones (e.g., cholecystokinin, enteroglucagon, gastrin) neurotransmitters of the enteric nervous system (e.g., vasoactive intestinal polypeptide, calcitonin gene-related peptide, substance $\mathrm{P}$ ), paracrine and autocrine factors (e.g., prostaglandins, nitric oxide, endothelin, angiotensin II, serotonin), as well as absorption of specific nutrients (164-166).

These mechanisms, either alone or in combination, reduce intestinal arterial resistance in an effort to preserve intestinal oxygenation during conditions which either compromise intestinal oxygen delivery or enhance tissue oxygen consumption. Intrinsic regulation of blood flow limits the reduction in intestinal perfusion in the face of moderately severe hypotension (pressure-flow autoregulation), during continuous adrenergic stimulation (escape phenomenon), and during moderate hypoxaemia (167-169). In addition, the metabolic mechanism and other locally released mediators are involved in initiating the increase in intestinal blood flow in response to the ingestion of a meal (postprandial hyperaemia) $(165,170,171)$.

Information regarding the development of the intrinsic regulation of intestinal perfusion is based on observations made in neonatal swine. It was proposed that intrinsic regulation in the neonatal gut is predominantly exerted by metabolic feedback mechanisms. Escape from prolonged sympathetic nerve stimulation $(172,173)$, and postprandial hyperaemia were present in the gut of 3-day old swine (174-176). The neonatal gut also demonstrated an increase in arteriovenous oxygen content difference as well as an increase in capillary exchange capacity during periods of hypotension or hypoxia $(176,177)$. In contrast, myogenic control of arterial tone and pressure-flow autoregulation were absent in the neonatal intestinal circulation (175,178-180). As a result, moderate hypoxaemia or hypotension were accompanied by a considerably smaller increase in intestinal blood flow in newborn swine than in older swine $(158,178,181,182)$. These data suggest that the neonatal intestine is prone to hypoxic injury during conditions which reduce intestinal perfusion pressure and/or arterial oxygen content, since oxygen delivery to the tissue can only be maintained by increasing tissue oxygen extraction by opening capillary exchange vessels $(183,184)$.

Based on the aforementioned studies it was proposed that the maturation of the intrinsic regulation of intestinal arterial tone depends on postnatal age. However, several limitations preclude the interpretation of these studies with regard to the maturation of intrinsic vascular control. These studies were conducted in neonatal animals born at term, and may therefore not be applicable to the developing gut. In addition, many of the observations were done in isolated intestinal loops, which may not correctly represent the in vivo situation. Moreover, since total intestinal blood flow was determined, these studies do not provide information on the 
exact site and mechanisms of intrinsic control of intestinal vascular tone, as well as the developmental changes herein.

\section{Role of the circulation in intestinal complications}

From the previous sections it is clear that the intestinal blood supply is regulated by two systems which affect the intestinal arterial diameter in opposite directions. Extrinsic mechanisms decrease the intestinal arterial diameter, whereas intrinsic mechanisms increase the arterial diameter. It follows that the blood supply to the intestinal tissue is ultimately determined by the balance between constriction and dilation of intestinal arteries and arterioles. A disturbance in this balance may lead to insufficient intestinal blood supply and, consequently, intestinal hypoxia.

Intestinal hypoxia causes structural and functional changes in the adult gut, such as mucosal atrophy, decreased motor activity, and increased intestinal permeability, leading to loss of intestinal nutritive and barrier functions. Such a sequence of events may also contribute to the initiation and/or progression of intestinal complications in preterm neonates, including NEC, as indicated by the presence of coagulation necrosis in surgical specimens of affected bowel $(185,186)$. Although the intestinal necrosis is sometimes clearly related to insults which obviously affect the oxygenation of the gut, such as mesenteric thromboembolism, antenatal cocaine abuse, polycythaemia, and exchange transfusion, in most cases of NEC no such trigger events have occurred.

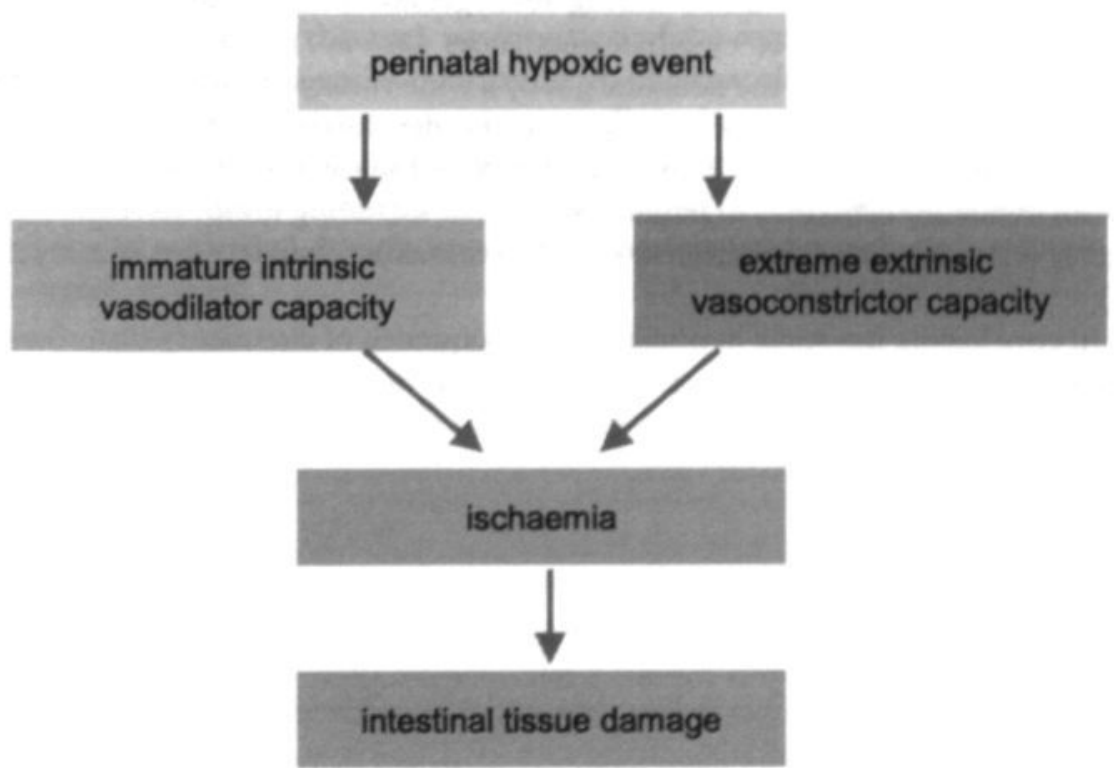

Figure 4. Common hypotheses on the role of circulatory disturbances in necrotising enterocolitis 
Two major theories have been postulated regarding the origin of circulatory disturbances in the pathophysiology of NEC (Figure 4). According to the classic theory, perinatal events induce an intense constriction of the splanchnic vasculature, resulting in a severe reduction of intestinal blood flow or even complete ischaemia, eventually causing hypoxic tissue damage $(77,187,188)$. Potential perinatal events implied in the pathogenesis of NEC include birth asphyxia, respiratory distress syndrome, persistent ductus arteriosus, and congenital heart disease (189-194).

An alternative theory poses that immaturity of intrinsic vasodilator regulation in the splanchnic circulation leads to insufficient perfusion of the developing gut $(183,184)$. As reviewed in the previous section, metabolic and myogenic vasodilator mechanisms are only present to a limited extent in the newborn gut. In contrast to older neonates and adults, which attempt to maintain gut oxygenation by increasing both blood flow and tissue oxygen extraction, the newborn gut can only increase oxygen extraction during periods of decreased oxygen delivery. Therefore, the immature gut is prone to compromised oxygenation under circumstances such as hypoxaemia and/or hypotension.

In addition to hypoxia itself, it has been proposed that subsequent restoration of oxygen supply to the intestine may produce ongoing injury to the intestinal tissue by initiating an inflammatory response (195-198). Release of reactive oxygen metabolites and inflammatory mediators, such as platelet-activating factor and tumor necrosis factor-alpha, during hypoxia-reoxygenation has been implied in the pathogenesis of intestinal damage in experimental animal models of NEC (199-204). Moreover, it has been shown in adult animals that accumulation of activated leukocytes into the tissue during reoxygenation may aggravate intestinal damage, via the release of cytotoxic mediators (205-207). The initiation of an inflammatory response in the neonatal gut by a wide variety of precipitating events may represent a final common pathway for the development of intestinal injury (208). This is supported by the finding of elevated plasma levels of a number of pro-inflammatory cytokines in patients with NEC, including tumor necrosis factor, platelet activating factor, interferon- $\gamma$, and interleukin-6 and $-8(200,202,204,209$ 211).

In conclusion, intestinal hypoxia as a consequence of circulatory disturbances appears to play a role in the pathophysiology of intestinal complications in preterm neonates. However, the exact nature of such disturbances remains to be elucidated. 
Data regarding the circulatory physiology in the developing intestine are virtually nonexisting. Progression in this field is hampered by the lack of an adequate animal model. We therefore designed a model for studying the circulation in the developing intestine of the chick foetus in vivo at consecutive stages during the second half of foetal development by means of intravital videomicroscopy (Chapter 5).

The chick foetus was selected as the experimental animal for several reasons. First, the chick foetus has been demonstrated to be a valid model for studying circulatory physiology (156). With regard to the intestine, development of the human gut is more closely related to that of precocial species (pig, chick, guinea pig) as compared with that of altricial species (rat, mouse). Second, the intestine of the chick foetus is partly located outside the abdomen in its natural omphalocele until two days before hatching. This renders the intestinal circulation readily accessible without elaborate invasive surgery and anaesthesia, thereby avoiding any influence of anaesthetics on arterial tone. Third, foetal haemodynamic parameters are not influenced by maternal factors, since the chick foetus develops independently of its mother within the eggshell. Nutrients are provided to the foetus by the albumen and yolk-sac compartments, whereas gas exchange is provided by the chorioallantoic membrane, a highly vascularised membrane which lines the inside of the eggshell and is regarded as the avian homologue of the mammalian placenta.

The main objective of the experimental studies was to elucidate the development of the intrinsic and extrinsic mechanisms controlling blood flow to the immature intestine. To this end, we investigated the regulation of vascular tone in mesenteric resistance arteries in response to pharmacological stimuli. In addition, we determined vasomotor responses as well as changes in heart rate and blood pressure during acute hypoxia (Chapter 6). Subsequently, we studied the role of oxygen in the establishment of local vasomotor and central haemodynamic control (Chapter 7). A detailed description of the experimental procedures is given in the respective chapters. 
1. Trahair J.T. Is fetal enteral nutrition important for normal gastrointestinal growth?: a discussion. JPanenter Enter Nutr 1993; 17: 82-85.

2. Surana R. and Puri P. Small intestinal atresia: effect on fetal nutrition. J Pediatr Surg 1994; 29. $1250-1252$.

3. Blakelock R., Upadhyay V., Kimble R., Pease P., Kolbe A., and Harding J. Is a normally functioning gastrointestinal tract necessary for normal growth in late gestation? Pediatr Sung Int 1998; 13: 17-20.

4. Gluckman P.D. and Heyman M.A., editors. Pediatrics and Perinatology, The Scientific Basis. London: Arnold, 1996.

5. Epstein M.L and Rudolph C.D. Embryology and Anatomy of the Gastrointestinal Tract. In: Gluckman P.D. and Heymann M.A., editors. Pediatrics and Perinatology, the Scientific Basis. London: Arnold, 1996 . $597-601$.

6. Herbst J.J. Development of suck and swallow. J Pediatr Gastroenterol Nutr 1981; 2: 5131-5135.

7. Lebenthal E. Human Gastrointestinal Development. New York: Raven Press, 1989.

8. Ross M.G. and Nijland M.J.M. Development of ingestive behavior. Am J Physiol 1998; 274: R879-R893.

9. Jarocka-Cyrta E., Perin N., Keelan M., Wierzbicki E., Wierzbicki T., Clandinin M.T., and Thomson A.B.R. Early dietary experience influences ontogeny of intestine in response to dietary lipid changes in later life.Am J Physiol 1998; 275: G250-G258.

10. Pitkin R.M. and Reynolds W.A. Fetal ingestion and metabolism of amniotic fluid protein. Am J Obstet Gynecol 1975; 123: 356-363.

11. Ritkin R.M. and Reynolds W.A. Fetal ingestion and metabolism of amniotic fluid protein. Am J Obstet Gynecol 1975; 123: 356-363.

12. Gitlin D., Kumate J., Morales C., Noriega L., and Arevalo N. The turnover of amniotic fluid protein in the human conceptus. Am J Obstet Gynecol 1972; 113: 632-645.

13. Berseth C.L. Gestational evolution of small intestinal motility in preterm and term infants. J Pediatr 1989; 115: 1523-1528.

14. Berseth C.L. and Nordyke C.K. Manometry can predict feeding readiness in preterm infants. Gastroenterology 1992; 103: 1523-1528.

15. Kien C.L. Digestion, absorption, and fermentation of carbohydrates in the newborn. Clin Perinatol 1996; 23: 211-228.

16. Mayne A., Hughes C.A., Sule D., Brown G.A., and McNeish A.S. Development of intestinal disaccharidases in preterm infants. Lancet 1983; 2: 622-623.

17. Auriochio S., Rubino A., and Murset G. Intestinal glycosidase activities in the human embryo, fetus, and newborn. Pediatrics 1965; 35:944-954.

18. Antonowicz I., Chang S.K., and Grand R.J. Development and distribution of lysosomal enzymes and disaccharidases in human fetal intestine. Gastroenterology 1974; 67:51-58.

19. Antonowicz I. and Lebenthal $\mathrm{E}$. Developmental pattern of small intestinal enterokinase and disaccharidase activities in the human fetus. Gastroenterology 1977; 72: 12991303.

20. Neu J. and Koldovsky 0. Nutrient absorption in the preterm neonate. Clin Perinatol 1996; 23: 229243.

21. Raul F., Lacroix B., and Aprahamian M. Longitudinal distribution of brush border hydrolases and morphological maturation in the intestine of the preterm infant. Early Human Dev 1986; 13: 225-234.

22. Auricchio S. and Sebastio G. Development of Disaccharidases. In: Lebenthal E, editor. Human Gastrointestinal Development. New York: Raven Press, 1989: 451-470.

23. Hamosh M. Digestion in the newborn. Clin Perinatol 1996; 23: 191-209. 
24. Lebenthal E. and Lee P.C. Development of functional responses in human exocrine pancreas. Audiatrics 1980; $166: 556-560$.

25. Wright E.M., Hirayama B.A., Loo D.D.F., Turk E, and Hager K. Intestinal Sugar Transport. In: Johnson LR, editor. Physiology of the Gastrointestinal Tract. 3rd ed. New York: Raven Press, 1994: 1751-1772.

26. Schmitz J. Digestive and Absorptive Function. In: Walker W.A., Durie P.R., Hamilton J.R., WalkerSmith JA., and Watkins J.B., editors. Pediatric Gastrointestinal Disease. Philadelphia: B.C. Decker Inc., 1991: 266-280.

27. Koldovsky 0. Development of Absorption of Monosaccharides. In: Lebenthal E, editor. Human Gastrointestinal Development. New York: Raven Press, 1989: 437-449.

28. Malo $\mathrm{C}$. Separation of two distinct $\mathrm{Na}^{+} / \mathrm{D}$-glucose cotransport systems in the human fetal jejunum by means of their differential specificity for 3-0-methylglucose. Biochim Biophys Acta 1990; 1022: 8-16.

29. McNeish A.S., Mayne A, and Ducker D.A. Development of D-glucose absorption in the perinatal period. J Pediatr Gastmenterol Nutr 1983; 2 (suppl): S222-S226.

30. Malo $\mathrm{C}$. and Berteloot A. Proximo-distal gradient of $\mathrm{Na}^{+}$-dependent D-glucose transport activity in the brush border membrane vesicles from the human fetal small intestine. FEBS Lett 1987; 220: 201-205.

31. Borgstrom B., Lindquist B., and Lundh G. Enzyme concentration and absorption of protein and glucose in duodenum of premature infants. Am J Dis Child 1960;99. 338-343.

32. Menard D., Monfils S., and Tremblay E. Ontogeny of human gastric lipase and pepsin activities. Gastroenterology 1995; 108: 1650-1656.

33. Levy E., Thibault $\mathrm{L}_{\text {, }}$, and Menard D. Intestinal lipids and lipoproteins in the human fetus: modulation by epidermal growth factor. J Lipid Res 1992; 33: 1607-1617.

34. Jarvenpaa A.L. Feeding the low-birth-weight infant. IV. Fat absorption as a function of diet and duodenal bile acids. Pediatrics 1983; 72:684689.

35. Agunod M., Yamaguchi N., Lopez R., Luhby A.L., and Glass G.B. Correlative study of hydrochloric acid, pepsin, and intrinsic factor secretion in newborns and infants. Am J Dig Dis 1969, 14: 400-414.

36. Kolacek S., Puntis J.W., Lloyd D.R., Brown G.A., and Booth I.W. Ontogeny of pancreatic exocrine function. Anch Dis Child 1990; 65: 178-181.

37. Boehm G., Bierbach U., DelSanto A., Moro G., and Minoli L. Activities of trypsin and lipase in duodenal aspirates of healthy preterm infants: effects of gestational and postnatal age. Biol Neonate 1995; 67: 248-253.

38. Auricchio S., Stellato A., and De Vizia B. Development of brush border peptidases in human and rat small intestine during fetal and neonatal life. Pediatr Res 1981; 15:991-995.

39. Malo C. Multiple pathways for amino acid transport in brush border membrane vesicles isolated from the human fetal small intestine. Gastroenterology 1991; 100: 1644-1652.

40. Sagawa N., Nishimura T., Ogawa M., and Inouye A. Electrogenic absorption of sugars and amino acids in the small intestine of the human fetus. Membr Biochem 1979, 2:393-404.

41. Walker W.A. Gastrointestinal defence: importance of gut closure in control of macromolecular transport. Ciba Found Symp 1979, 70: 201-216.

42. Sanderson I.R. and Walker W.A. Uptake and transport of macromolecules by the intestine: possible role in clinical disorders (an update). Gastroenterology 1993; 104:622639.

43. Weaver LT. and Walker W.A. Epidermal growth factor and the developing human gut. Gastroenterology 1988, 94: 845-847.

44. Menard D. Growth-promoting Factors and the Development of the Human Gut. In: Lebenthal E, editor. Human Gastrointestinal Development. New York: Raven Press, 1989. 123-150.

45. Jakobsson L, Axelsson L, Juvonen P., Lindberg T., and Lothe L. Human alpha-lactalbumin as a marker of macromolecular absorption in early infancy. Acta Paediatr Scand Suppl 1989, 351: $42-47$. 
46. Roberton D.M., Paganelli R., Dinwiddie R., and Levinsky R.J. Milk antigen absorption in the preterm and term neonate. Arch Dis Child 1982; 57: 369372.

47. Weaver L.T. and Walker W.A. Uptake of Macromolecules in the Neonate. In: Lebenthal E, editor. Human Gastrointestinal Development. New York: Raven Press, 1989. 731-748.

48. Blakey J.L., Lubitz L., Barnes G.L., Bishop R.F., Campbell N.T., and Gillam G.L. Development of gut colonisation in pre-term neonates. J Med Microbiol 1982; 15: 519529.

49. Millar M.R., MacKay P., Levene M., Langdale V., and Martin C. Enterobacteriaceae and neonatal necrotsing enterocolitis. Anch Dis Child 1992; 67: 53-56.

50. Fryklund B., Tullus K., Berglund B., and Burman L.G. Importance of the environment and the faecal flora of infants, nursing staff and parents as sources of gram-negative bacteria colonizing newborns in three neonatal wards. Infection 1992; 20: 253-257.

51. Dai D.W. and Walker W.A. Role of bacterial colonization in neonatal necrotizing enterocolitis and is prevention. Acta Paed Sin 1998; 39: 357-365.

52. Gewolb I.H., Schwalbe R.S., Taciak V.L., Harrison T.S., and Panigrahi P. Stool microflora in extremely lov birthweight infants. Anch Dis Child Fetal Neonatal Ed 1999; 80: F167-F173.

53. Mackie R.I., Sghir A., and Gaskins H.R. Developmental microbial ecology of the neonatal gastrointestinal tract. Am J Clin Nutr 1999; 69 (suppl): 1035S-1045S.

54. Stark P.L. and Lee A. The bacterial colonization of the large bowel of pre-term low birth weight neonates JHyg Camb 1982; 89: 5967.

55. Fryklund B, Tullus K, and Burman L. E. Epidemiology of enteric bacteria in neonatal units - influenoc of procedures and patient variables. J Hosp Inf 1991; 18: 15-21.

56. Savey A., Fleurette J., and Salle B.L. An analysis of the microbial flora of premature neonates. J Hosp Inf 1992; 21: 275-289.

57. Perman J.A. Gastrointestinal Flora: Developmental Aspects and Effects on Nutrients. In: Lebenthal E, editor. Human Gastrointestinal Development. New York: Raven Press, 1989: 777-786.

58. Dai D.W. and Walker W.A. Protective nutrients and bacterial colonization in the immature human gut. Adv Pediatr 1999; 46: 353-382.

59. Sakata H., Yoshioka H., and Fujita K. Development of the intestinal flora in very low birth weight onfants compared to normal full-term newborns. Eur J Pediatr 1985; 144: 186-190.

60. Israel E.J. Neonatal necrotizing enterocolitis, a disease of the immature intestinal mucosal barrier. Acta Paediatr Suppl 1994; 396: 27-32.

61. Kleinman R.E. and Walker W.A. The development of barrier function of the gastrointestinal tract. Acta Paediatr Scand Suppl 1989; 351: 3437.

62. Clancy J. and Klein R. Development of Gastrointestinal Tract Cellular Immunity. In: Lebenthal E, editor. Human Gastrointestinal Development. New York: Raven Press, 1989: 699708.

63. Insoft R.M., Sanderson I.R., and Walker W.A. Development of immune function in the intestine and its role in neonatal diseases. Pediatr Clin North Am 1996; 43:551-571.

64. Goldman A.S. Modulation of the gastrointestinal tract of infants by human milk. Interfaces and interactions. An evolutionary perspective. J Nutr 2000; 130: 426S-431S.

65. Sherman P.M., Forstner J.F., and Forstner G.G. Mucosal Barrier and its Defense During the Perinatal Period. In: Lebenthal E, editor. Human Gastrointestinal Development. New York: Raven Press, 1989. 687-698.

66. Bloom P.D. and Boedecker E.C. Mucosal immune responses to intestinal bacterial pathogens. Semin Gastrointest Dis 1996; 7: 151-166.

67. Madara J.L. Loosening tight junctions, lessons from the intestine. J Clin Invest 1989; 83: 10891094.

68. Madara J.L. Tight junctions dynamics: is paracellular transport regulated? Cell 1988; 53: 497-498. 
69. McKay D.M. and Baird A.W. Cytokine regulation of epithelial permeability and ion transport. Gut 1999; 44: 283-289.

70. Stevenson B.R. Understanding tight junction clinical physiology at the molecular level. J Clin Imvest $1999,104: 3-4$.

71. Madara J.L. Regulation of the movement of solutes across tight junctions. Annu Rev Physiol 1998; 60: 143-159.

72. Nusrat A., Turner J.R., and Madara J.L. Molecular physiology and pathophysiology of tight junctions. IV. Regulation of tight junctions by extracellular stimuli: nutrients, cytokines, and immune cells. Am J Physiol 2000; 279: G851-G857.

73. Boccia D., Stolfi L., Lana S., and Moro M.L. Nosocomial necrotising enterocolitis outbreaks: epidemiology and control measures. Eur J Pediatr 2001; 160: 385-391.

74. Hentschel J., De Veer L., Gastmeier P., Ruden H., and Obladen M. Neonatal nosocomial infection surveillance: incidences by site and a cluster of necrotizing enterocolitis. Infection 1999, 27: $234-238$.

75. Gregersen N., Van Nierop W., Von Gottberg A., Duse A., Davies V., and Cooper P. Klebsiella pneumoniae with extended spectrum beta-lactamase activity associated with a necrotizing enterocolitis outbreak. Pediatr Infect Dis J 1999, 18: $963-967$.

76. Faix R.G. and Adams J.T. Neonatal necrotizing enterocolitis: current concepts and controversies. Adv Pediatr Inf Dis 1994; 9: 1-36.

77. Kliegman R.M. and Fanaroff A.A. Necrotizing enterocolitis. N Engl J Med 1984; 310: 1093-1103.

78. Albanese C.T. and Rowe M.I. Necrotizing enterocolitis. Semin Pediatr Surg 1995; 4: 200-206.

79. Ahtonen P., Lehtonen 0.P., Kero P., Eerola E., and Hartiala K. Clostridium perfringens in stool, intrapartum antibiotics and gastrointestinal signs in a neonatal intensive care unit. Acta Paediatr 1994; 83: 389390.

80. Scheifele D.W. Role of bacterial toxins in neonatal necrotizing enterocolitis. / Pediatr 1990; 117: S44S51.

81. Lawrence G., Bates J., and Gaul A. Pathogenesis of neonatal necrotising enterocolitis. Lancel 1982; 1: 137-139.

82. Van Acker J., De Smet F., Muyldermans G., Bougatef A., Naessens A., and Lauwers S. Outbreak of necrotizing enterocolitis associated with enterobacter sakazakii in powdered milk formula.J Clin Microbiol 2001; 39: 293-297.

83. Peter C.S., Feuerhahn M., Bohnhorst B., Schlaud M., Ziesing S., von der Hardt H., and Poets C.F. Necrotising enterocolitis: is there a relationship to specific pathogens? Eur J Pediatr 1999, 158: 67-70.

84. Szylit 0., Butel M.J., and Rimbault A. An experimental model of necrotising enterocolitis. Lancet 1997; 350: 33-34.

85. Butel M.J., Roland N., Hibert A., Popot F., Favre A., Tessedre A.C., Bensaada M., Rimbault A., and Szylit 0. Clostridial pathogenicity in experimental necrotising enterocolitis in gnotobiotic quails and protective role of bifidobacteria. JMed Micmbiol 1998; 47: $391-399$.

86. Committee on Nutrition A.A. Nutritional needs for low-birth-weight infants. Pediatrics 1985; 75: 976-986.

87. La Gamma E.F. and Browne L.E. Feeding practices for infants weighing less than $1500 \mathrm{~g}$ at birth and the pathogenesis of necrotizing enterocolitis. Clin Perinatol 1994; 21: 271-305.

88. Gross S.J. and Slagle TA. Feeding the low birth weight infant. Clin Perinatol 1993; 20: 193-209.

89. Adan D., La Gamma E.F., and Browne L.E. Nutritional management and the multisystem organ failure / systemic inflammatory response syndrome in critically ill preterm neonates. Crit Care Clin 1995; 11: 751-784.

90. Pereira G.R. Nutritional care of the extremely premature infant. Clin Perinatol 1995; 22:61-75. 
91. Heird W.C. and Gomez M.R. Parenteral nutrition. In: Tsang RC, Lucas A, Uauy R, Zlotkin S, editors. Nutritional Needs of the Preterm Infant; Scientific Basis and Practical Guidelines. New York: Caduceus Medical Publishers, 1993: 225-242.

92. Newell S.J. Enteral feeding of the micropremie. Clin Perinatol 2000; 27: 221-234.

93. Schanler R.J. The Low-Birth-Weight Infant. In: Walker WA, Watkins JB, editors. Nutrition in Pediatrics; Basic Science and Clinical Applications. 2nd ed. Hamilton: B.C. Decker Inc., 1997: $392-412$.

94. Hay W.W., Lucas A., Heird W.C., Ziegler E., Levin E., Grave G.D., Catz C.S., and Yaffe S.J. Workshop sum mary: nutrition of the extremely low birth weight infant. Pediatrics 1999; 104: 1360-1368.

95. Pinchasik D. From TPN to breast feeding - feeding the premature infant - 2000: Part I. Parenteral nutrition. Am J Perinatol 2001; 18: $59-72$.

96. Berseth C.L. Neonatal small intestinal motility: motor responses to feeding in term and preterm infants. JPediatr 1990; 117: 777-782.

97. Berseth C.L. Gastrointestinal motility in the neonate. Clin Perinatol 1996; 23: 179190.

98. Greer F.R. Feeding the premature infant in the 20th century. J Nutr 2001; 131: 426S-430S.

99. Atkinson S.A. Human milk feeding of the micropremie. Clin Perinatol 2000; 27: 235-247.

100. Lucas A. and Cole T.J. Breast milk and neonatal necrotising enterocolitis. Lancet 1990; 336: 1519-1523.

101. Schanler R.J., Hurst N.M., and Lau C. The use of human milk and breastfeeding in premature infants. Clin Perinatol 1999; 26: 379398.

102. Schanler R.J., Shulman R.J., and Lau C. Feeding strategies for premature infants: beneficial outcomes of feeding fortified human milk versus preterm formula. Pediatrics 1999; 103: 1150-1157.

103. Amin S.B., Merle K.S., Orlando M.S., Dalzell L.E., and Guillet R. Brainstem maturation in premature infants as a function of enteral feeding type. Pediatrics 2000; 106: 318-322.

104. Lucas A., Morley R., Cole T.J., Lister G., and Lesson-Payne C. Breast milk and subsequent intelligence quotient in children born preterm. Lancet 1992; 339: 261-264.

105. Singhal A., Cole T., and Lucas A. Early nutrition in preterm infants and later blood pressure: two cohorts after randomised trials. Lancet 2001; 357: 413-419.

106. Buescher E.S. Host defense mechanisms of human milk and their relations to enteric infections and necrotizing enterocolitis. Clin Perinatol 1994; 21: 247-261.

107. Rodriguez-Palmero M., Koletzko B., Kunz C., and Jensen R. Nutritional and biochemical properties of human milk (part II): Lipids, micronutrients, and bioactive factors. Clin Perinatol 1999; 26: 335-360.

108. Garofalo R.P. and Goldman A.S. Expression of functional immunomodulatory and anti-inflammatory factors in human milk. Clin Perinatol 1999; 26: 361-377.

109. Kunz C., Rodriguez-Palmero M., Koletzko B., and Jensen R. Nutritional and biochemical properties of human milk (part I): General aspects, proteins, and carbohydrates. Clin Perinatol 1999; 26: 307-333.

110. Schanler RJ. Suitability of human milk for the low-birthweight infant. Clin Perinatol 1995; 22: 207-222.

111. Barrett Reis B., Hall R.T., Schanler R.J., Berseth C.L., Chan G., Ernst J.A., Lemons J., Adamkin D., Baggs G., and $O^{\prime}$ Connor D. Enhanced growth of preterm infants fed a new powdered human milk fortifier: a randomized, controlled trial. Pediatrics 2000; 106: 581-588.

112. Moody G.J., Schanler R.J., Lau C., and Shulman R.J. Feeding tolerance in premature infants fed fortified human milk. J Panenter Enter Nutr 2000; 30: 408-412.

113. Lucas A., Morley R., and Cole TJ. Randomised trial of early diet in preterm babies and later intelligence quotient. BMJ 1998; 317: 1481-1487.

114. Trahair J.F. and Harding R. Restitution of swallowing in the fetal sheep restores intestinal growth after midgestation esophageal obstruction. J Pediatr Gastroenterol Nutr 1995; 20: 156-161. 
115. Trahair J.F. and Sangild P.T. Systemic and luminal influences on the perinatal development of the gut. Equine Vet J 1997; 24: 40-50.

116. Buchmiller T.L., Gregg J., Rivera F.A., Diamond J.M., and Fonkalsrud E.W. Effect of esophageal ligation on the development of fetal rabbit intestinal lactase. J Pediatr Sung 1993; 28: 1473-1477.

117. Mulvihill S.J., Stone M.M., Debas H.T, and Fonkalsrud E.W. The role of amniotic fluid in fetal nutrition. JPediatr Sung 1985; 20: 668672.

118. Trahair J.F., Harding R., Bocking A.D., Silver M., and Robinson P.M. The role of ingestion in the development of the small intestine in fetal sheep. Q J Exp Physiol 1986; 71:99-104.

119. Buchmiller T.L., Fonkalsrud E.W., Kim C.S., Chopourian H.L., Shaw K.S., Lam M.M., and Diamond J.M. Upregulation of nutrient transport in fetal rabbit intestine by transamniotic substrate administration. J Sung Res 1992; 52: 443-447.

120. Chartton-Char V. and Rudolph A.M. Digestion and absorption of carbohydrates by the fetal lamb in utero. Pediatr Res 1979, 13: 1018-1023.

121. Castillo R.O., Pittler A., and Costa F. Intestinal maturation in the rat: the role of enteral nutrients. JPanenter Enter Nutr 1988; 12: 490-495.

122. Buchman A.L., Moukarzel A.A., Bhuta S., Belle M., Ament M.E., Eckhert C.D., Hollander D., Gornbein J., Kopple J.D., and Vijayaroghavan S.R. Parenteral nutrition is associated with intestinal morphologic and functional changes in humans. J Panenter Enter Nutr 1995; 19: 453-460.

123. MacFie J. Enteral versus parenteral nutrition: the significance of bacterial translocation and gut-barrier function. Nutrition 2000; 16:606-611.

124. Berseth C.L. Minimal enteral feedings. Clin Perinatol 1995; 22: 195-205.

125. Lucas A., Bloom S.R., and Aynsley-Green A. Gut hormones and minimal enteral feeding Acta Paediatr Scand 1986; $75: 719723$.

126. Shulman R.J., Schanler R.J., Lau C., Heitkemper M., Ou C.-N., and O'Brian Smith E. Early feeding, antenatal glucocorticoids, and human milk decrease intestinal permeability in preterm infants. Pediatr Res 1998; $44: 519-523$.

127. Shulman R.J., Schanler R.J., Lau C., Heitkemper M., Ou C.-N., and O'Brian Smith E. Early feeding feeding intolerance, and lactase activity in preterm infants. J Pediatr 1998; 133:645-649.

128. Berseth C.L. Effect of early feeding on maturation of the preterm infant's small intestine.J Pediatr 1992; 120:947-953.

129. Berseth C.L and Nordyke C. Enteral nutrients promote postnatal maturation of intestinal motor activity in preterm infants. Am J Physiol 1993; 264: G1046-G1051.

130. McClure R.J. and Newell S.J. Randomised controlled trial of trophic feeding and gut motility. Anch Dis Child Fetal Neonatal Ed 1999, 80: F54-F58.

131. Dunn L., Hulman S., Weiner J., and Kliegman R. Beneficial effects of early hypocaloric enteral feeding on neonatal gastrointestinal function: preliminary report of a randomized trial. J Pediatr 1988; 112 : 622629 .

132. Troche B., Harvey-Wilkes K., Engle W.D., Nielsen H.C., Frantz L.D., Mitchell M.L., and Hermos R.J. Early minimal enteral feedings promote growth in critically ill premature infants. Biol Neonate 1995; 67: 172-181.

133. Meetze W.H., Valentine C., MoGuigan J.E., Conlon M., Sacks N., and Neu J. Gastrointestinal priming prior to full enteral nutrition in very low birth weight infants $J$ Pediatr Gastroenterol Nutr 1992; 15: $163-170$.

134. McClure R.J. and Newell S.J. Randomised controlled study of clinical outcome following trophic feeding Anch Dis Child Fetal Neonatal Ed 2000, 82: F29F33. 
135. Tyson J.E. and Kennedy K.A. Minimal enteral nutrition in parenterally fed neonates. In: Sinclair JC, Bracken MB, Soll RF, Horbar JD, editors. Neonatal module of The Cochrane Database of Systematic Reviews. Oxford, 1997.

136. Rowe M.I., Reblock K.K., Kurkchubasche A.G., and Healey P.J. Necrotizing enterocolitis in the extremely low birth weight infant. J Pediatr Surg 1994; 29: 987-991.

137. La Gamma E.F., Ostertag S.G., and Birenbaum H. Failure of delayed oral feedings to prevent necrotizing enterocolitis; results of study in very-low-birth-weight neonates. Am J Dis Child 1985; 139: 385-389.

138. Willis D.M., Chabot J., Radde I.C., and Chance G.W. Unsuspected hyperosmolality of oral solutions contributing to necrotizing enterocolitis in very-low-birth-weight infants. Pediatrics 1977; 60: 535-538.

139. Clark D.A. and Miller M.J.S. Intraluminal pathogenesis of necrotizing enterocolitis. J Pediatr 1990; 117: S64-S67.

140. Clark D.A., Thompson J.E., Weiner L.B., McMillan J.A., Schneider A.J., and Rokahr J.E. Necrotizing enterocolitis: intraluminal biochemistry in human neonates and a rabbit model. Pediatr Res 1985; 19 : 919921.

141. Crissinger K.D. and Tso P. The role of lipids in ischemia/reperfusion-induced changes in mucosal permeability in developing piglets. Gastroenterology 1992; 102: 1693-1699.

142. Kliegman R.M., Walker W.A., and Yolken R.H. Necrotizing enterocolitis: research agenda for a disease of unknown etiology and pathogenesis. Pediatr Res 1993; 34: 701-708.

143. Murray R.D. Effects of bacterial fermentation end products on intestinal function: implications for intestinal dysfunction. J Pediatr 1990; 117: S59-S63.

144. Casley-Smith J.R. and Gannon B.J. Intestinal Microcirculation: Spatial Organization and Fine Structure. In: Shepherd AP, Granger DN, editors. Physiology of the Intestinal Circulation. New York: Raven Press, 1984: 931 .

145. Kreulen D.L. and Keef K.D. Electrophysiological and Neuromuscular Relationships in Extramural Blood Vessels. In: Schultz S.G., Wood J.D., Rauner B.B., editors. Handbook of Physiology; Section 6: The Gastrointestinal System, Volume 1, Part 2. Bethesda: American Physiological Society, 1989: 1605-1634.

146. Thompson R.J., Jackson A., and Nurse C.A. Developmental loss of hypoxic chemosensitivity in rat adrenomedullary chromaffin cells. J Physiol 1997; 489: 503-510.

147. Rychkov G.Y., Adams M.B., McMillen I.C., and Roberts M.L. Oxygen-sensing mechanisms are present in the chromaffin cells of the sheep adrenal medulla before birth. J Physiol 1998; 509: 887-893.

148. Inoue M., Fujishiro N., and Imanaga I. Hypoxia and cyanide induce depolarization and catecholamine release in dispersed guinea-pig chromaffin cells. J Physiol 1998; 507: 807-818.

149. Jacobson E.D. The Splanchnic Circulation. In: Johnson L.R., editor. Gastrointestinal Physiology. 4th ed. St.Louis: Mosby-Year Book, 1991: 142.

150. Jensen A., Hohmann M., and Kuenzel W. Dynamic changes in organ blood flow and oxygen consumption during acute asphyxia in fetal sheep. J Dev Physiol 1987; 9: 543-559.

151. Iwamoto H.S. Cardiovascular Effects of Acute Fetal Hypoxia and Asphyxia. In: Hanson M.A., Spencer J.A.D., Rodeck C.H., editors. Fetus and Neonate: Physiology and Clinical Applications. Volume 1: The Circulation. Cambridge: Cambridge University Press, 1993: 197-214.

152. Reuss M.L., Parer J.T., Harris J.L., and Krueger T.R. Hemodynamic effects of alpha-adrenergic blockade during hypoxia in fetal sheep. Am J Obstet Gynecol 1982; 142: 410-415.

153. Jensen $\mathrm{A}$. and Lang $\mathrm{U}$. Foetal circulatory responses to arrest of uterine blood flow in sheep: effects of chemical sympathectomy. J Dev Physiol 1992; 17: 75-86.

154. Iwamoto H.S., Kaufman T., Keil L.C., and Rudolph A.M. Responses to acute hypoxemia in fetal sheep at 0.6-0.7 gestation. Am J Physiol 1989; 256: H613-H620. 
155. Mulder A.L.M., Van Golde J.C., Prinzen F.W., and Blanco C.E. Cardiac output distribution in response to hypoxia in the chick embryo in the second half of the incubation time.JPhysiol 1998; 508: 281-287.

156. Van Golde J.C. Chick embryo as a model in fetal physiology. Thesis, University of Maastricht, 1999.

157. Buckley N.M., Diamant S., Frasier L.D., and Owusu K. Histamine or adenosine blockade alters intestinal blood flow autoregulation in swine. Am J Physiol 1988; 254: G156-G161.

158. Nowicki P.T., Miller C.E., and Haun S.E. Effects of arterial hypoxia and isoproterenol on in vitro postnatal intestinal circulation. Am J Physiol 1988; 255: H1144-H1148.

159. Granger H.J. and Nyoff R.A. Dynamics of intestinal axygenation: interactions between oxygen supply and uptake. Am J Physiol 1982; 243: G91-G96.

160. Granger D.N., Kvietys P.R., and Perry M.A. Role of exchange vessels in the regulation of intestinal axyge nation. Am J Physiol 1982; 242: 6570-6574.

161. Davis M.J. and Hill M.A. Signaling mechanisms underlying the vascular myogenic response. Physiol Rev 1999, 79. 387-423.

162. Johnson P.C. Autoregulation of blood flow. Cinc Res 1986; 59: 483-495.

163. Meininger G.A. and Davis M.J. Cellular mechanisms involved in the vascular myogenic response. Am JPhysiol 1992; 263: H647-H659.

164. Hansen M.B., Dresner L.S., and Wait R.B. Profile of neurohumoral agents on mesenteric and intestinal blood flow in health and disease. Physiol Res 1998; 47: 307-327.

165. Matheson P.J., Wilson M.A., and Garrison R.N. Regulation of intestinal blood flow. J Sung Res 2000; 93: 182-196.

166. Nowicki P.T. Postnatal changes in gut hemodynamics: a possible role for substance P.Am / Physiol 1998; 274: G1142-G1150.

167. Chen L.Q. and Shepherd A.P. Role of $\mathrm{H}^{+}$and alpha2-receptors in escape from sympathetic vasoconstriction. Am J Physiol 1991; 261: H868-H873.

168. Chen L.Q., Riedel G.L., and Shepherd A.P. Norepinephrine release during autoregulatory escape: effects of alpha2-receptor blockade. Am J Physiol 1991; 260: H400-H408.

169. Shepherd A.P. Local control of intestinal oxygenation and blood flow. Annu Rev Physiol 1982; 44: 13-27.

170. Sawmiller D.R. and Chou C.C. Role of adenosine in postprandial and reactive hyperemia in canine jejunum. Am J Physiol 1992; 263: G487-G493.

171. Bohlen H.G. Integration of intestinal structure, function, and microvascular regulation. Microcincula tion 1998; 5: 27-37.

172. Nowicki P.T., Miller C.E., and Hayes J.R. Effect of sustained mesenteric nerve stimulation on intestinal axygenation in developing swine. Am J Physiol 1991; 260: G333-G339.

173. Crissinger K.D., Kvietys P.R., and Granger D.N. Autoregulatory escape from norepinephrine infusion: roles of adenosine and histamine. Am J Physiol 1988; 254: G560-G565.

174. Crissinger K.D. and Burney D.L. Postprandial hemodynamics and oxygenation in developing pigjet intestine. Am J Physiol 1991; 260: G951-G957.

175. Nowicki P.T. and Miller C.E. Effect of increased tissue oxygen uptake on autoregulation in postnatal intestine. Am J Physiol 1992; 263: G690-G694.

176. Crissinger K.D. and Burney D.L. Influence of luminal nutrient composition on hemodynamics and axygenation in developing intestine. Am J Physiol 1992; 263: G254G260.

177. Nowicki P.T. and Miller C.E. Regulation of capillary exchange capacity in postnatal swine intestine. Am JPhysiol 1993; 265: G1090-G1097.

178. Nowicki P.T., Miller C.E., and Edwards R.C. Effects of hypoxia and ischemia on autoregulation in postnatal intestine. Am JPhysiol 1991; 261: G152-G157. 
179. Crissinger K.D., Kvietys P.R., and Granger D.N. Developmental intestinal vascular responses to venous pressure elevation. Am JPhysiol 1988; 254: G658-G663.

180. Nowicki P.T. and Miller C.E. Autoregulation in the developing postnatal intestinal circulation. Am JPhysiol 1988; 254: G189G193.

181. Szabo J.S., Stonestreet B.S., and Oh W. Effects of hypoxemia on gastrointestinal blood flow and gastric emptying in the newborn piglet. Pediatr Res 1985; 19: 466-471.

182. Nowicki P.T., Hansen N.B., Hayes J.R., Menke J.A., and Miller R.R. Intestinal blood flow and $\mathrm{O}_{2}$ uptake during hypoxemia in the newborn piglet. Am J Physiol 1986; 251: G19G24.

183. Nowicki P.T. and Nankervis C.A. The role of the circulation in the pathogenesis of necrotizing enterocolitis. Clin Perinatol 1994; 21: 219-233.

184. Crissinger K.D. Regulation of hemodynamics and oxygenation in developing intestine: insight into the pathogenesis of necrotizing enterocolitis. Acta Paediatr Suppl 1994; 396: 8-10.

185. Ballance W.A., Dahms B.B., Shenker N., and Kliegman R.M. Pathology of neonatal necrotizing enterocolitis: a ten-year experience. J Pediatr 1990; 117: S6-S13.

186. Santulli T.V., Schullinger J.N., Heird W.C., Gongaware R.D., Wigger J., Barlow B., Blanc W.A., and Berdon W.E. Acute necrotizing enterocolitis in infancy: a review of 64 cases. Pediatrics 1975; 55: 376-387.

187. Lloyd J.R. The etiology of gastrointestinal perforations in the newborn.J Pediatr Surg 1969; 4: 77-84.

188. Touloukian R.J., Posch J.N., and Spencer R. The pathogenesis of ischemic gastroenterocolitis of the neonate: selective gut mucosal ischemia in asphyxiated neonatal piglets. J Pediatr Surg 1972; 7 : $194-204$.

189. Kosloske A.M. A unifying hypothesis for pathogenesis and prevention of necrotizing enterocolitis. JPediatr 1990; 117: S68-S74.

190. Leung M.P., Chau K.T., Hui P.W., Tam A.Y.C., Chan F.L., Lai C.L., and Yeung C.Y. Necrotizing enterocolitis in neonates with symptomatic congenital heart disease.J Pediatr 1988; 113: 1044-1046.

191. Touloukian R.J., Kadar A., and Spencer R.P. The gastrointestinal complications of neonatal umbilical venous exchange transfusion: a clinical and experimental study. Pediatrics 1973; 51:36-43.

192. Leake R.D., Thanopoulos B., and Nieberg R. Hyperviscosity syndrome associated with necrotizing enterocolitis. Am J Dis Child 1975; 129; 1192-1194.

193. Bunton G.L., Durbin G.M., McIntosh N., Shaw D.G., Taghizadeh A., Reynolds E.O.R., Rivers R.P.A., and Urman G. Necrotizing enterocolitis; controlled study of 3 years' experience in a neonatal intensive care unit. Am J Dis Child 1977; 52: 772-777.

194. McElhinney D.B., Hedrick H.L., Bush D.M., Pereira G.R., Stafford P.W., Gaynor J.W., Spray T.L., and Wernovsky G. Necrotizing enterocolitis in neonates with congenital heart disease: risk factors and outcomes. Pediatrics 2000; 106: 1080-1087.

195. Crissinger K.D. and Granger D.N. Mucosal injury induced by ischemia and reperfusion in the piglet intestine: influences of age and feeding, Gastroenterology 1989; 97: 920-926.

196. Crissinger K.D., Grisham M.B., and Granger D.N. Developmental biology of oxidant-producing enzymes and antioxidants in the piglet intestine. Pediatr Res 1989; 25:612-616.

197. MacKendrick W. and Caplan M. Necrotizing enterocolitis; new thoughts about pathogenesis and potential treatments. Pediatr Clin North Am 1993; 40: 1047-1059.

198. Clark E.S., Crissinger K.D., and Granger D.N. Oxidant-induced increases in mucosal permeability in developing piglets. Pediatr Res 1990; 28: 28-37.

199. Caplan M.S., Sun X.-M., and Hsueh W. Hypoxia causes ischemic bowel necrosis in rats: the role of plateletactivating factor (PAF-acether). Gastmenterology 1990; 99: 979986.

200. Caplan M.S., Sun X.-M., Hsueh W., and Hageman J.R. Role of platelet activating factor and tumor necrosis factor-alpha in neonatal necrotizing enterocolitis. J Pediatr 1990; 116: $960-964$. 
201. Hsueh W., Caplan M.S., Sun X., Tan X., MacKendrick W., and Gonzalez-Crussi F. Platelet-activating factor, tumor necrosis factor, hypoxia and necrotizing enterocolitis. Acta Paediatr Suppl 1994; 396: 11-17.

202. Ford H., Reblock K., and Rowe M. Role of inflammatory cytokines and nitric oxide in the pathogenesis of necrotizing enterocolitis. J Pediatr Sung 1997; 32: 275-282.

203. Muguruma K., Gray P.W., Tjoelker LW., and Johnston J.M. The central role of PAF in necrotizing enterocolitis development. Eicos 1997; 3:379-382.

204. Rabinowitz S.S., Dzakpasu P., Piecuch S., Leblanc P., Valencia G., and Kornecki E. Platelet-activating factor in infants at risk for necrotizing enterocolitis. J Pediatr 2001; 138: 81-86.

205. Park P.O., Haglund U., Bulkley G.B., and Falt K. The sequence of development of intestinal tissue injury after strangulation ischemia and reperfusion. Surgery 1990; 107: 574-580.

206. Simpson R., Alon R., Kobzik L., Valeri C.R., Shepro D., and Hechtman H.B. Neutrophil and nonneutrophil-mediated injury in intestinal ischemia-reperfusion. Ann Surg 1993; 218: 444-454.

207. Kurtel H., Tso P., and Granger D.N. Granulocyte accumulation in postischemic intestine: role of leukocyte adhesion glycoprotein CD11/CD18. Am J Physiol 1992; 262: G878-G882.

208. Caplan M.S. and MacKendrick W. Inflammatory mediators and intestinal injury. Clin Porinatol 1994; 21: 235-245.

209. Morecroft J.A., Spitz L., Hamilton P.A., and Holmes S.J.K. Plasma cytokine levels in necrotizing enterocolitis. Acta Paediatr Suppl 1994; 396: 18-20.

210. Edelson M.B., Bagwell C.E., and Rozycki H.J. Circulating pro- and counterinflammatory cytokine levels and severity in necrotizing enterocolitis. Pediatrics 1999, 103: 766-771.

211. Harris M.C., Costarino A.T., Sullivan J.S., Dulkerian S., MoCawley L, Corcoran L., Butler S., and Kilpatrick L. Cytokine elevations in critically ill infants with sepsis and necrotizing enterocolitis. J Pediatr 1994; 124: $105-111$. 


\section{CHAPTER 3}

Intestinal Permeability and Carrier-mediated Monosaccharide Absorption in Preterm Neonates during the Early Postnatal Period

De beste methode om de dingen met succes te onderzoeken, is hun verloop te vervolgen vanaf het begin.

(Aristoteles, $4^{e}$ eeuw v. Chr.) 


\section{Abstract}

Objective. Immaturity of intestinal epithelial barrier function and absorptive capacity may play a role in the pathophysiology of intestinal complications in preterm neonates during the early postnatal period. We determined the intestinal permeability and carrier-mediated absorption of monosaccharides in preterm neonates during the first two weeks after birth.

Study design. Fifty-nine preterm neonates born between 25 and 32 weeks gestation were included within 24 hours of birth. Neonates received exclusively parenteral nutrition during the first 7 days after birth; enteral feeding was initiated at day 8 . An intestinal permeability-absorption test was performed at 1, 4, 7, and 14 days after birth. The lactulose/rhamnose ratio was determined as a marker of intestinal permeability. Urinary excretion percentages of D-xylose and 3-0-methyl-D-glucose were determined as markers of passive and active carrier-mediated monosaccharide absorption, respectively.

Results. Intestinal permeability transiently increased between day 1 and 7 in all neonates $(p<0.05)$. Carriermediated monosaccharide absorption increased between day 1 and 14 in neonates of $28-30 \mathrm{wk}(p<0.05)$ to the level observed in the neonates of 30-32 wk gestation. In neonates $<28 \mathrm{wk}$, intestinal permeability at day 7 was higher $(p<0.05)$ and carrier-mediated monosaccharide absorption at day 14 was lower $(p<0.01)$ as compared with the older neonates.

Conclusions. The barrier function of the intestinal epithelium transiently decreases during the first week after birth in preterm neonates which are not enterally fed. Diminished barrier function and low monosaccharide absorptive capacity, particularly in neonates $<28 \mathrm{wk}$, may predispose these patients to the development of intestinal complications during the early postnatal period. 


\section{INTRODUCTION}

Gastrointestinal complications, such as feeding intolerance, necrotising enterocolitis, and gut-associated sepsis, pose a considerable problem in the care of preterm neonates. At present, necrotising enterocolitis is the most common surgical emergency and a major cause of death in this patient population (1-3). These intestinal complications occur mostly in the first weeks after birth, suggesting that they result from failure of the immature intestine to adapt adequately to the transition from intrauterine to extrauterine life.

Birth induces two major changes in the luminal environment of the intestine. First, after delivery the neonatal gut is exposed to microorganisms and their products. To prevent host invasion by potentially pathogenic microorganisms, a full barrier function of the intestinal wall is required. Second, the intravenous nutrient supply via the placental-umbilical circulation is interrupted after birth. Consequently, the neonate depends on the gastrointestinal tract for the acquisition of nutrients through the processes of propulsion, digestion, and absorption of ingested food.

The anatomical differentiation of the human foetal gut is already completed by 20 weeks gestation (4). However, mature levels of digestive enzyme secretion are not reached until the end of foetal gestation $(5,6)$. Lactase activity at 34 weeks gestation is only $30 \%$ of the level in the full-term newborn (7). Furthermore, intestinal motor activity in response to luminal nutrients is not present until 31 weeks, and is still immature at 40 weeks gestation (8). Thus, the development of various functions of the intestine lag behind its structural development.

Little is known about the development of the barrier function as well as the absorptive function of the human gut. Intestinal barrier function is in part dependent on the close interaction of intact adjacent epithelial cells, which impedes the diffusion of luminal substances. This property of the epithelium can be assessed indirectly by determining the intestinal permeability to orally administered watersoluble molecules, such as lactulose and $\mathrm{L}$-rhamnose $(9,10)$. It has previously been shown in preterm neonates that the permeability changes during the first month after birth, and that it may be affected by enteral nutrition (11-13). However, these studies did not investigate the permeability during the immediate postnatal period, and/or did not discern between preterm neonates of different gestational ages. The aim of the current study was therefore to investigate the intestinal permeability in preterm neonates born at 25 to 32 weeks gestation during the first 14 days after birth. Next, we studied the capacity of the intestinal epithelium to absorb monosaccharides by both passive and active carrier-mediated transport mechanisms, by measuring the urinary excretion of orally administered D-xylose and 3-O-methyl-Dglucose in these neonates. In order to investigate the influence of enteral nutrients on intestinal permeability and monosaccharide absorption, these parameters were studied during a one-week period of exclusive parenteral nutrition as well as after the initiation of enteral feeding. 


\section{METHODS}

\section{Patients and study design}

The study was conducted in the neonatal intensive care unit of the University Hospital Maastricht from July 1997 to September 1999, and was approved by the local medical ethics committee. Parents of eligible neonates were informed in detail about the study, and written informed consent was obtained before enrollment.

Preterm neonates admitted to the intensive care unit were eligible for the study if they were born at a gestational age of 25-32 weeks and if postnatal age was less than 24 hours. Criteria for exclusion included: major congenital anomalies, severe asphyxia (defined as 5-minute Apgar score $\leq 3$ ), persistent hypoxaemia or respiratory acidosis, and severe hypotension. For each neonate we recorded clinical characteristics at study entry, including gestational age, birth weight, sex, Apgar score at 1 and 5 minutes, CRIB score (Clinical Risk Index for Babies) (14), presence of asphyxia, mode of delivery, prolonged rupture of the membranes (>24 h), chorioamnionitis, (pre)eclampsia/HELLP syndrome, antenatal administration of corticosteroids, tocolysis, and multiple pregnancy. We furthermore recorded the occurrence of sepsis, defined as clinical signs of sepsis and positive blood culture.

In order to investigate the influence of enteral nutrients on intestinal permeability and monosaccharide absorption, the study was implemented in two phases. Between day 1 and 7 after birth, neonates received only intravenous fluids. Intravenous fluids consisted of $10 \%$ glucose/calcium solution for the first day, and of total parenteral nutrition thereafter. In general, initial fluid intake was $80 \mathrm{ml} / \mathrm{kg}$ / day and was increased by $20 \mathrm{ml} / \mathrm{kg} /$ day up to approximately $150 \mathrm{ml} / \mathrm{kg} /$ day. This volume was adapted on the basis of clinical evaluation, urine production, weight measurement, and serum electrolyte values. From day 8 after birth onwards, neonates received enteral feeding in addition to parenteral nutrition. Enteral nutrition consisted of either mothers' own milk (obtained and used within 48 hours) or full strength $(24 \mathrm{kcal} / 30 \mathrm{ml})$ preterm formula (Nenatal, Nutricia, Zoetermeer, the Netherlands), according to the choice of the parents. Enteral feeding was administered as bolus feedings via a nasogastric tube at an initial volume of $12 \mathrm{ml} /$ day, and increased until complete enteral nutrition (approximately $150 \mathrm{ml} / \mathrm{kg} /$ day) was achieved. The rate of enteral feeding increment was determined by the attending physician and adapted as required by the infants' clinical condition. In case of feeding intolerance, as defined by emesis, large gastric residuals, abdominal distension, and/or ileus, enteral feedings were reduced or withheld until the problem resolved. Non-bloody gastric residuals of less than $3 \mathrm{ml}$ per 2 hours were refed.

\section{Assessment of intestinal permeability and monosaccharide absorption}

To allow the simultaneous evaluation of intestinal permeability and monosaccharide absorption, we conducted a sugar permeability-absorption test at day 1, 4, 7, and 14 after birth. The sugar solution consisted of $8.6 \mathrm{~g}$ lactulose (Centrafarm, Etten-Leur, the Netherlands), $140 \mathrm{mg}$ L-rhamnose (Acros Organics, Pittsburgh, PA), $70 \mathrm{mg}$ D-xylose (Genfarma, Maarssen, the Netherlands), and $140 \mathrm{mg}$ 3-O-methylD-glucose (Sigma, St. Louis, MO) dissolved in $100 \mathrm{ml}$ demineralised water (425 mOsm/l). Each test day, $2 \mathrm{ml}$ of the sugar solution was administered to the neonate 
via the nasogastric tube. All urine passed in the next 4 hours was collected in an adhesive urine bag (Urinocol Premature, Braun Biotrol, Paris, France). At day 14, no enteral feeding was given in the $\mathbf{2}$ hours preceding and following ingestion of the test solution These time points were chosen to minimise interference with routine nursery protocols of the NICU. The complete 4-hour urine volume was measured and a $2 \mathrm{ml}$ aliquot was stored at $-80^{\circ} \mathrm{C}$ until analysis. The sugar absorption test critically relies on a complete urine collection. Therefore, in case urine collection failed in an infant, the sugar absorption test was repeated the next day. If urine sampling failed again, then measurements for the infant at this particular test day were regarded as missing values. Urinary concentrations of lactulose, L-rhamnose, D-xylose, and 3-O-methyl-D-glucose were determined by gas-liquid chromatography as previously described (15). This test is noninvasive, requires only minimal handling, and can be used safely in preterm neonates (16).

The four saccharides used in this test cross the intestinal epithelium via different pathways and are cleared by the kidneys. The percentage of the orally administered dose of each saccharide which is excreted in the urine thus reflects the functional state of the particular intestinal permeation pathway. Lactulose is a disaccharide which crosses the intestinal epithelium by passive diffusion through the paracellular tight junctions. L-rhamnose is a monosaccharide which crosses the intestinal epithelium mainly by transcellular passive diffusion through aqueous pores. The urinary excretion percentages of lactulose and rhamnose are markers for paracellular and transcellular diffusion, respectively. To correct for nonmucosal factors which may affect the intestinal uptake of these saccharides, including the rate of gastric emptying, intestinal transit time, and renal clearance, the urinary excretion percentages of lactulose and rhamnose were expressed as the lactulose/ rhamnose excretion ratio ( $L / R$ ratio). Since nonmucosal factors will affect urinary excretion of both saccharides to a similar extent, the $L / R$ ratio provides a reliable index of the permeability of the intestinal epithelium $(9,10)$. D-xylose and $3-0-$ methyl-D-glucose are monosaccharides which are absorbed by the intestinal epithelial cells via passive and active carrier-mediated transport mechanisms, respectively. The urinary recovery of orally administered xylose and methylglucose are markers for passive and active carrier-mediated monosaccharide absorption, respectively $(17,18)$.

\section{Measurement of urinary D-lactate concentration}

To investigate whether an increase in intestinal permeability to sugar probes was associated with an increase in the permeation of other substances present in the intestinal lumen, we measured the urinary excretion of D-lactate. This stereoisomer of L-lactate is derived from fermentation of unabsorbed carbohydrates by bacteria within the gut lumen, is neither produced nor metabolised by mammalian cells, and is excreted by the kidneys. Therefore, urinary D-lactate excretion is considered to reflect bacterial production and intestinal uptake (19).

Urinary D-lactate was determined at day 1, 4, 7, and 14 after birth. Urine samples were stored in $1 \mathrm{ml}$ aliquots at $-80^{\circ} \mathrm{C}$ until measurement. D-lactate was measured by means of an enzymatic assay according to a method previously described (20). Briefly, thawed urine was deproteinised with perchloric acid and centrifuged. 
The supernatant was added to a $\mathrm{NAD}^{+}$-glycine-hydrazine solution to form pyruvatehydrazon coupled with the reduction of $\mathrm{NAD}^{+}$to $\mathrm{NADH}$ at a $\mathrm{pH}<9.0$. This reaction was catalysed by the enzyme D-lactate dehydrogenase. NADH was measured spectrophotometrically at $340 \mathrm{~nm}$. The D-lactate concentration (in $\mathrm{mmol} / \mathrm{l}$ ) was expressed relative to the creatinine concentration (in $\mathrm{mmol} / \mathrm{l}$ ) in the urine sample to yield the D-lactate/creatinine ratio.

\section{Data analysis}

Data are presented either as median and interquartile range (clinical parameters) or as mean \pm SEM (biochemical parameters). The overall effect in time was determined by a repeated measurements analysis using the GLM procedure in SPSS version 10. However, missing data at several points in time precluded the use of this procedure for the assessment of differences between gestational age groups. Therefore, subsequent comparisons within groups were made using the Wilcoxon signed ranks test. Comparisons between groups were made using the MannWhitney U test. Statistical significance was defined as p $<0.05$ (two-sided).

Table 1. Clinical characteristics of the patients

\begin{tabular}{|c|c|c|c|}
\hline Variable & $\begin{array}{c}\text { Group A } \\
\left(25-27^{+6} \mathrm{wk}\right) \\
n=18\end{array}$ & $\begin{array}{c}\text { Group B } \\
\left(28-29^{+6} \text { wk }\right) \\
n=24\end{array}$ & $\begin{array}{c}\text { Group C } \\
\text { (30-32 wk) } \\
n=17\end{array}$ \\
\hline & & & \\
\hline Birth weight (g) & $880(808-1006)$ & $1050(795-1240)$ & $1340(1105-1488)$ \\
\hline Female:male ( $\%$ female) & $12: 6(66.7)$ & $11: 13(45.8)$ & $8: 9(47.1)$ \\
\hline \multicolumn{4}{|l|}{ Apgar score } \\
\hline $1 \mathrm{~min}$ & $6(3.5-8)$ & $9(7-9)$ & $7(5-9)$ \\
\hline $5 \mathrm{~min}$ & $9(8-9)$ & $5(4-8)$ & $9(7.5-10)$ \\
\hline CRIB score & $1.5(1-7)$ & $1.5(1-5)$ & $1(0.5-1.5)$ \\
\hline Asphyxia (\%) & 16.7 & 16.7 & 17.6 \\
\hline Caesarean delivery (\%) & 38.9 & 62.5 & 58.8 \\
\hline Rupture of membranes $>24 \mathrm{~h}(\%)$ & 22.2 & 29.2 & 35.3 \\
\hline Chorioamnionitis (\%) & 16.7 & 4.2 & 0 \\
\hline (Pre)eclampsia/HELLP(\%) & 5.6 & 16.7 & 5.9 \\
\hline Antenatal corticosteroids (\%) & 83.3 & 54.2 & 47.1 \\
\hline Tocolysis (\%) & 72.2 & 37.5 & 52.9 \\
\hline Multiple pregnancy (\%) & 27.8 & 25 & 35.3 \\
\hline \multicolumn{4}{|l|}{ Sepsis (\%) } \\
\hline day 1 & 5.6 & 0 & 0 \\
\hline day 4 & 5.6 & 8.3 & 0 \\
\hline day 7 & 16.7 & 25 & 6.7 \\
\hline day 14 & 27.8 & 28.6 & 27.3 \\
\hline
\end{tabular}

- Values are percentage of patients except for birth weight, Apgar score, and CRIB score, which are median (interquartile range). 
Fifty-nine preterm neonates born between 25 and 32 weeks gestation were enrolled. Neonates were stratified for gestational age: $25-27^{+6}$ (group A, $n-18$ ), 28-29+6 (group B, $n-24$ ), and 30-32 weeks gestation (group C, $n-17$ ). Clinical characteristics of the infants are summarised in Table 1. The sugar permeability-absorption test was not successful in all neonates at all four time points, due to one of the following reasons: failure of complete urine collection, failure of chemical analysis, unstable clinical condition, or transfer to another hospital.

Repeated measurements analysis for the group of neonates as a whole demonstrated an increase in the excretion of lactulose over time $(\mathrm{p}-0.014)$. Subgroup analysis showed that lactulose excretion did not change significantly during the first 14 days after birth in group $A$, whereas it significantly increased as compared with day 1 at day $4(\mathrm{p}-0.033)$ and $7(\mathrm{p}-0.006)$ in group $\mathrm{B}$, and at day 7 $(\mathrm{p}-0.028)$ in group C (Fig. 1A).

Repeated measurements analysis of L-rhamnose excretion demonstrated a decrease over time for the whole group $(\mathrm{p}-0.011)$. The reduction in urinary $\mathrm{L}$ rhamnose excretion was statistically significant in group $\mathrm{A}$ at day $7(\mathrm{p}-0.038)$ and 14 $(\mathrm{p}-0.050)$, and in group $\mathrm{C}$ at day $4(\mathrm{p}-0.046)$. Rhamnose excretion at day 7 and 14 was significantly lower in group $\mathbf{A}$ as compared with the other two groups ( $\mathrm{p}-0.009$ and $\mathrm{p}=0.008$, respectively; Fig. 1B).

As a result of these changes in lactulose and rhamnose excretion, the $L / R$ ratio increased over time for the group as a whole ( $\mathrm{p}-0.001)$. Subgroup analysis demonstrated that the increase in $\mathrm{L} / \mathrm{R}$ ratio between day 1 and 7 was significant in all three groups ( $\mathrm{p}=0.018, \mathrm{p}=0.006, \mathrm{p}=0.046$ in group $\mathrm{A}, \mathrm{B}, \mathrm{C}$, respectively). This peak in $\mathrm{L} / \mathrm{R}$ ratio at day 7 was significantly higher in group $A$ as compared with the other two groups ( $\mathrm{p}-0.025$ ). The $\mathrm{L} / \mathrm{R}$ ratio declined between day 7 and 14 (Fig. 1C).

The pattern of urinary D-lactate excretion closely resembled that of the $L / R$ ratio (Fig. 2). Since no significant differences in the D-lactate/creatinine ratio were noted between the groups, data obtained in the three gestational age groups were pooled at each time point. The D-lactate/creatinine ratio was significantly higher at day 7 as compared with day $1(102 \pm 21.5$ vs. $48 \pm 10.3$, p -0.001$)$, and subsequently decreased at day 14 after birth $(60 \pm 13.8)$.

Repeated measurements analysis demonstrated an increase in the urinary excretion percentages of xylose and 3-O-methyl-D-glucose for the group of neonates as a whole ( $\mathrm{p}-0.007$ and $\mathrm{p}-0.028$, respectively). However, subgroup analysis showed that xylose excretion in group $\mathbf{A}$ did not change during the first two weeks, and was significantly lower as compared with the other two groups at day $4(\mathrm{p}-0.024)$ and $14(\mathrm{p}-0.002)$ after birth (Fig. 3A). In contrast, in group B, xylose excretion increased significantly at day $4(\mathrm{p}-0.011), 7$ ( $\mathrm{p}-0.005)$, and 14 ( $\mathrm{p}-0.036)$ as compared with day 1 . Xylose excretion was highest in group $\mathrm{C}$, and remained at a constant level during the study period.

The changes in the urinary excretion percentages of methylglucose parallelled those of xylose. In group A, methylglucose excretion did not significantly increase during the two weeks, and was significantly lower as compared with the other two groups at day $4(\mathrm{p}-0.043)$ and $14(\mathrm{p}-0.003)$ after birth (Fig. 3B). In group B, a 


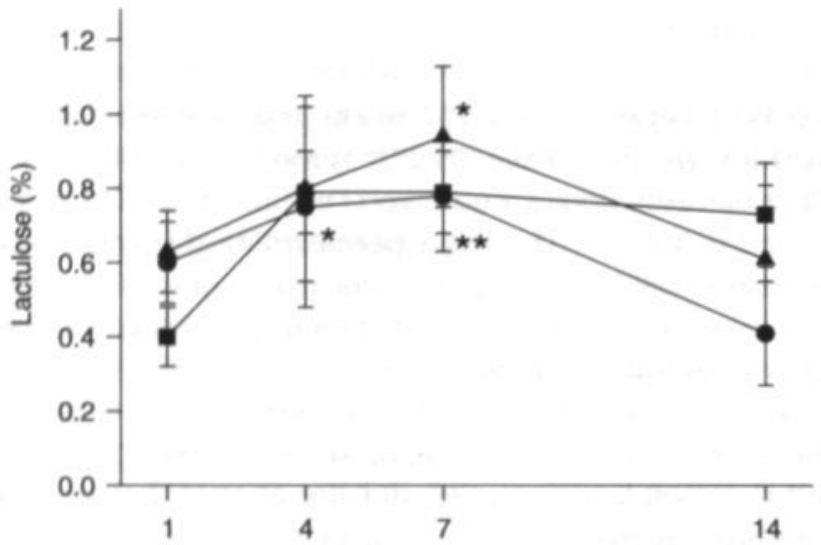

- $25-27^{46} \mathrm{wk}$

- $28-29^{+6} \mathrm{wk}$

- $30-32 \mathrm{wk}$

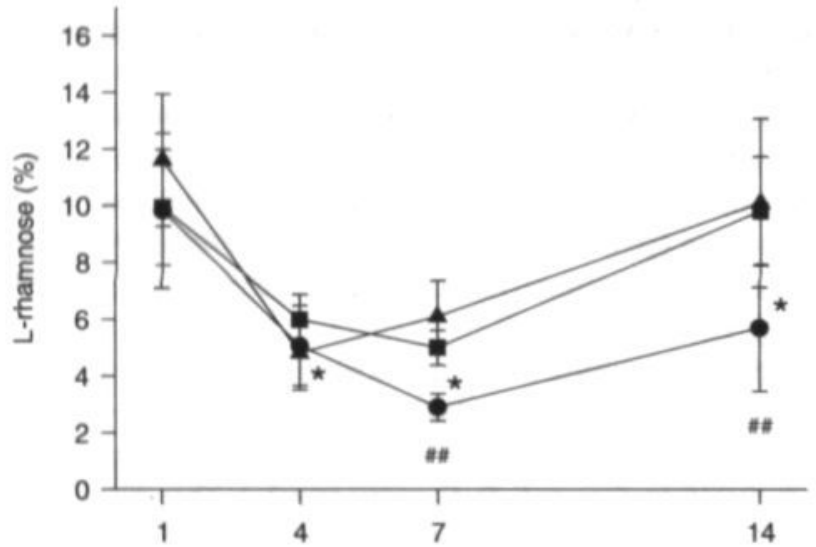

Figure 1A

Figure 1B

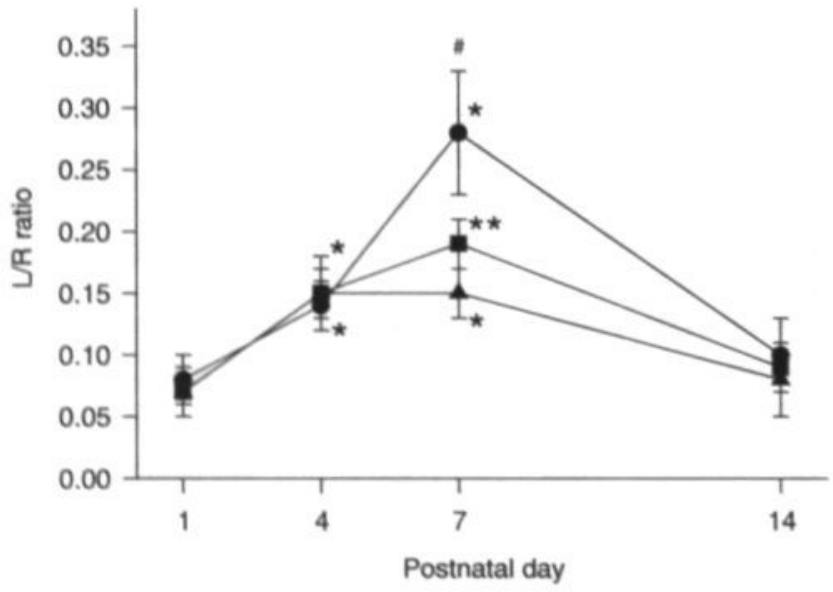

Figure 1C

\section{Figure 1}

Intestinal paracellular diffusion (A), transcellular diffusion (B), and permeability (C) as determined by the urinary excretion percentages of orally administered lactulose and L-rhamnose, and the lactulose / rhamnose ratio (L/R ratio), respectively, during the first two weeks after birth. Data are mean $\pm S E M ; n-8,12$, and 11 at day $1 ; n-9,13$, and 8 at day $4 ; n=10,15$, and 7 at day $7 ; n-11,10$, and 5 at day 14 .

"* p<0.05, p<0.01, vs. day 1; *, ** p<0.05, p<0.01, 28 wk vs. $\geq 28$ wk gestation 
significant increase in methylglucose excretion was observed at day $4(p-0.041)$, $7(p-0.016)$, and $14(p-0.017)$ as compared with day 1 . Urinary excretion of methylglucose at day 1 after birth was highest in group $C$, and remained at this level during the first 14 days after birth.

We also evaluated the influence of the clinical variables mentioned in Table 1 on the urinary excretion percentages of each of the four saccharides and on the lactulose/rhamnose ratio. We compared the neonates exposed to the particular variable with those which were not exposed. Comparisons were made for the group of neonates as a whole, since the numbers in the individual gestational age groups were too low to allow reliable statistical analysis. Only the L/R ratio at day 1 after birth was significantly higher in neonates whose mothers received steroids antenatally with those who did not $(0.079 \pm 0.01 \mathrm{vs}$. $0.039 \pm 0.01, \mathrm{p}-0.023)$. This difference was not continued at later time points. No statistically significant differences were detected with regard to the other factors or the type of enteral feeding.

\section{DISCUSSION}

The neonate depends on its intestine for protection against microorganisms and for absorption of ingested nutrients. To date, little is known about the development of the barrier and nutritive functions of the intestine in preterm neonates. In the present study, we investigated the changes in intestinal epithelial permeability and carrier-mediated absorption of monosaccharides in preterm neonates born between 25 and 32 weeks gestation. The data showed that intestinal permeability increased during the first week after birth, most markedly in neonates $<28$ weeks, and subsequently decreased during the second postnatal week after the initiation of enteral feeding. Both passive and active carrier-mediated monosaccharide absorption at day 14 after birth were higher in neonates born between 28 and 32 weeks as compared with those $<28$ weeks gestation.

Previous studies provide conflicting data with regard to neonatal intestinal permeability. Both decreases and increases in permeability during the first month after birth have been reported (11-13). Interpretation of the findings of these studies is further hampered by discrepancies in gestational age, clinical condition, feeding regimen, and postnatal age at study. We assessed the intestinal permeability at 1,4, 7 , and 14 days after birth in preterm neonates by determining the urinary lactulose/ L-rhamnose excretion ratio ( $/ / R$ ratio). The $L / R$ ratio at day 1 was similar in all neonates born between 25 and 32 weeks gestation. Although there are unfortunately no data available on intestinal permeability in older neonates using a similar lactulose/rhamnose test, the $L / R$ ratio in the current study was within the normal range previously reported for healthy infants and adults $(21,18)$. Together, this may suggest that a mature level of intestinal epithelial barrier function is already attained by 25 weeks gestation.

The increase in $\mathrm{L} / \mathrm{R}$ ratio during the first week after birth was in part due to a decrease in rhamnose excretion in all neonates, most markedly in those born between 25 and 28 weeks gestation. Since rhamnose crosses the intestinal epithelium predominantly via passive diffusion through small aqueous pores in the cell mem- 


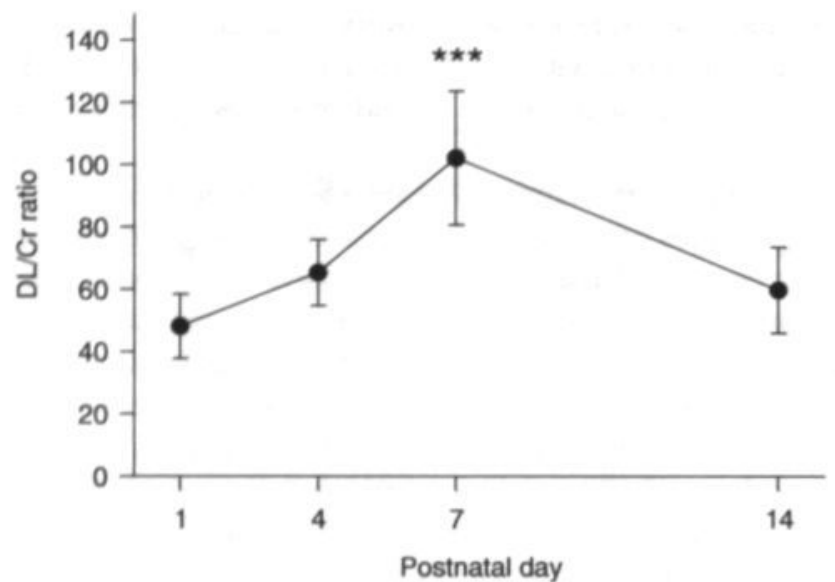

Figure 2

Urinary D-lactate excretion during the first two weeks after birth in neonates of 25-32 weeks gestation; D-lactate concentration is expressed relative to the creatinine concentration in the urine samples. Data are mean \pm SEM; $n-45,46$, 47 , and 38 at day $1,4,7$, and 14 , respectively.

$\cdots p<0.001$, vs. day 1

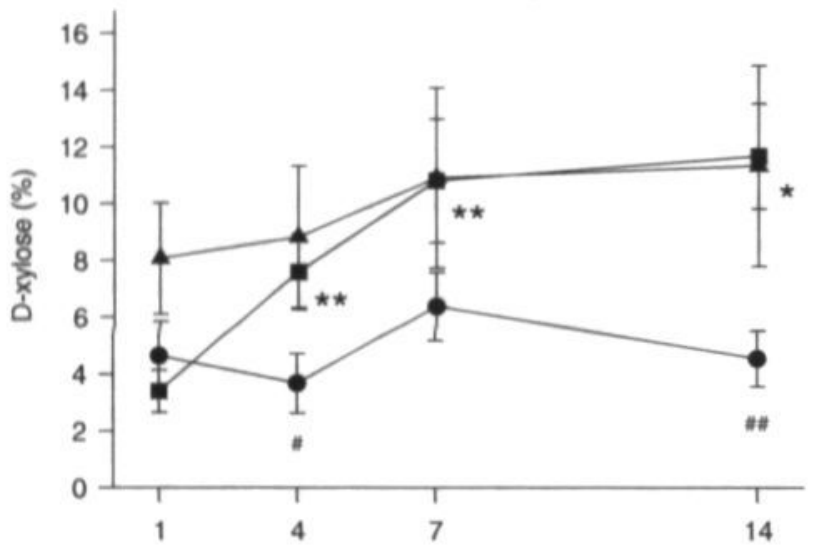

- $25-27^{+6} w k$
- $28-29^{+6} w k$
A $30-32 w k$

Figure 3A

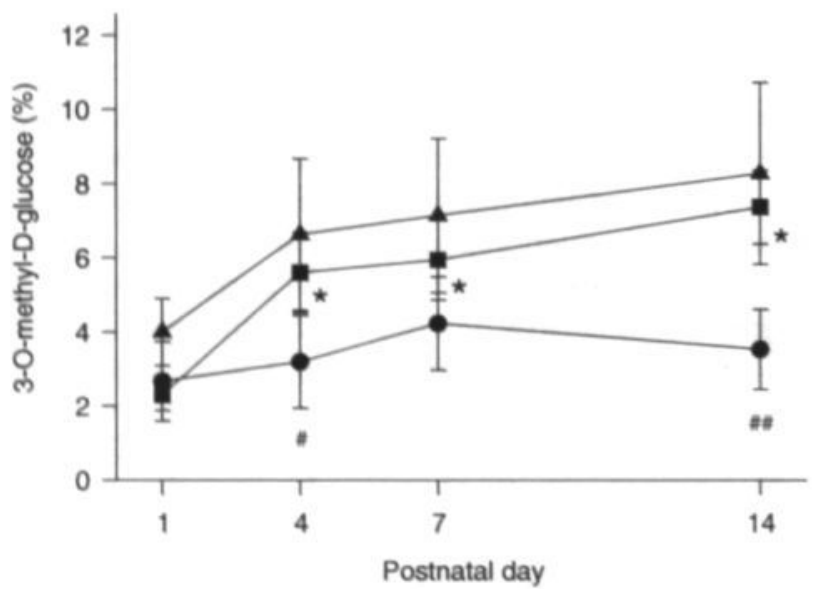

Figure 3B

Figure 3

Intestinal passive (A) and active (B) carrier-mediated monosaccharide absorption as determined by the urinary excretion percentages of orally administered $\mathrm{D}$-xylose and 3-0-methyl-D-glucose during the first two weeks after birth. Data are mean $\pm \mathrm{SEM} ; n=10,13$, and 11 at day $1 ; n-11,13$, and 8 at day $4 ; n-10,15$, and 8 at day $7 ; n-11,10$, and 5 at day 14 .

$\because, \cdots p<0.05, p<0.01$, vs. day $1 ; *$, * p $<0.05, p<0.01,<28$ wk vs. $\geq 28$ wk gestation 
branes of villus epithelial cells $(9,10)$, these data indicate that transcellular diffusion across the intestinal epithelium decreased during the first week after birth. In neonates $>28$ weeks gestation, the increase in $L / R$ ratio also resulted from an increase in lactulose excretion. Since lactulose permeates the intestinal mucosa via paracellular diffusion through the tight junction complexes between adjacent epithelial cells $(9,10)$, these data indicate that the leakiness of the tight junctions had increased at day 7 after birth in these neonates.

Interestingly, the $\mathrm{L} / \mathrm{R}$ ratio declined after the initiation of enteral feeding during the second week of life. This suggests that the increase in intestinal permeability during the first postnatal week was at least partly related to the absence of nutrients in the intestinal lumen. Similar increases in permeability have been found in adult patients for whom total parenteral nutrition is the sole nutritional source (22). Furthermore, it has been demonstrated that enteral starvation of a previously normal gut is accompanied by jejunal mucosal hypoplasia (23). A reduction of intestinal villous height may lead to a diminished mucosal surface area for the diffusion of rhamnose, while at the same time facilitating the diffusion of lactulose in the crypt regions (24). In addition, other factors, such as the release of cytokines and intestinal hypoxia during periods of intestinal hypoperfusion, may contribute to the increase in intestinal permeability by attenuating epithelial tight junction functioning $(25,26)$.

The clinical significance of an increase in intestinal permeability for sugar probes is still a subject of investigation. Although the assumption has been made that alterations in gut barrier function as assessed by changes in intestinal permeability predispose to bacterial translocation and septic complications, there is yet no evidence in humans to support this view $(27,28)$. A direct relation between increased permeability and enhanced passage of luminal factors has thus far only been demonstrated for IgA immune complexes, ovalbumin, and bacterial chemotactic peptides (29-31).

The rise in $L / R$ ratio between day 1 and 7 was accompanied by an increase in urinary D-lactate excretion. D-lactate is derived from the fermentation of unabsorbed carbohydrates by bacteria present in the gut lumen (19). Therefore, urinary D-lactate excretion may increase as a consequence of enhanced production and/or increased permeation of the substance across the intestinal epithelium. Since total bacterial counts in the neonatal gut continue to increase during the first month after birth (32), it is unlikely that the transient peak at day 7 was due to increased bacterial production. The increase in D-lactate excretion therefore most likely resulted from increased intestinal uptake, thereby supporting the hypothesis that increased intestinal permeability enhances the transmucosal passage of substances from the gut lumen.

In addition to the permeability of the intestinal epithelium, we also assessed the early postnatal development of passive and active carrier-mediated monosaccharide absorption. To this end, we measured the urinary excretion percentages of D-xylose and 3-O-methyl-D-glucose, respectively, the absorption of which mainly occurs in the proximal small intestine $(33,34)$. Interestingly, in neonates born between 28 and 30 weeks, absorption of these monosaccharides rapidly increased to the levels of the older neonates at the end of the first postnatal week, even in 
the absence of enteral nutrients. In contrast, in neonates born between 25 and 28 weeks, the absorption of xylose and methylglucose remained considerably lower throughout the first two weeks after birth. These changes in intestinal uptake of xylose and methylglucose over time could not be explained by alterations in preor postmucosal factors, including gastric emptying, intestinal transit, or renal clearance, as this would have affected the excretion of these two monosaccharides and rhamnose equally. Taken together, our data indicate that the capacity of the gut to absorb monosaccharides by passive as well as active carrier-mediated transport mechanisms is very limited in neonates born at less than 28 weeks gestation throughout the early postnatal period.

To our knowledge, this is the first study which provides insight into the early postnatal development of the barrier function of the intestinal epithelium as well as of the sugar absorptive capacity in preterm neonates. Our data indicate that premature exposure of the immature intestine to the extrauterine environment in the absence of enteral nutrients is associated with a reduction in epithelial integrity. Both a diminished barrier function and a low absorptive capacity during the early postnatal period, particularly in neonates born at less than 28 weeks gestation, may underlie the high vulnerability of these patients to intestinal complications. Given the finding that epithelial integrity was restored upon the initiation of enteral feeding, early administration of enteral nutrition may offer an effective strategy to support the intestinal adaptation to extrauterine life in preterm neonates.

\section{ACKNOWLEDGMENTS}

We thank all the families for their participation in this study, and the staff of the neonatal intensive care unit, Mr. B. Panis, Mrs. A. Gerver, and the department of Clinical Chemistry for their assistance. 


\section{References}

1. Kosloske A.M. Epidemiology of necrotizing enterocolitis. Acta Paediatr Suppl 1994; 396: $2-7$.

2. Uauy R.D., Fanaroff A.A., Korones S.B., Phillips E.A., Phillips J.B., and Wright L.L. Necrotizing enterocolitis in very low birth weight infants: biodemographic and clinical correlates. J Pediatr 1991; 119. 630-638.

3. Albanese C.T. and Rowe M.I. Necrotizing enterocolitis. Semin Pediatr Sung 1995; 4: 200-206.

4. Lebenthal E., Heitlinger L.A., and Lee P.C. Development of the gastrointestinal tract.J Pediatr 1983; 102: $1-9$.

5. Hamosh M. Digestion in the newborn. Clin Perinatol 1996; 23: 191-209.

6. Lebenthal E. Human Gastrointestinal Development. New York: Raven Press, 1989.

7. Lebenthal A. and Lebenthal E. The ontogeny of the small intestinal epithelium. J Panenter Enter Nutr 1999; 23: S3-S6.

8. Berseth C.L. Gastrointestinal motility in the neonate. Clin Perinatol 1996; 23: 179-190.

9. Bjarnason L., MacPherson A., and Hollander D. Intestinal permeability: an overview. Gastroentemlogy 1995; 108: 1566-1581.

10. Travis S. and Menzies L. Intestinal permeability: functional assessment and significance. Clin Sci 1992; 82: $471-488$.

11. Weaver L.T., Laker M.F., and Nelson R. Intestinal permeability in the newborn. Anch Dis Child 1984; 59: 236-241.

12. Beach R.C., Menzies LS., Clayden G.S., and Scopes J.W. Gastrointestinal permeability changes in the preterm neonate. Anch Dis Child 1982; 57: 141-145.

13. Shulman R.J., Schanler R.J., Lau C., Heitkemper M., Ou C.N., and O'Brian Smith E. Early feeding, antenatal glucocorticoids, and human milk decrease intestinal permeability in preterm infants. Pediatr Res 1998; $44: 519523$.

14. The International Neonatal Network. The CRIB (clinical risk index for babies) score: a tool for assessing initial neonatal risk and comparing performance of neonatal intensive care units. Lancet 1993; 342: 193-198.

15. Shippee R.L., Johnson A.A., Cioffi W.G., Lasko J., LeVoyer T.E., and Jordan B.S. Simultaneous determination of lactulose and mannitol in urine of burn patients by gas-liquid chromatography. Clin Chem 1992; 38: 343-345.

16. Piena M., Albers M.J.I.J., Van Haard P.M.M., Gischler S., and Tibboel D. Introduction of enteral feeding in neonates on extracorporeal membrane oxygenation after evaluation of intestinal permeability changes. J Pediatr Sung 1998; 33: 30-34.

17. Ohri S.K., Somasundaram S., Koak Y., MacPherson A., Keogh B.E., Taylor K.M., Menzies I.S., and Bjarnason $\mathrm{L}$. The effect of intestinal hypoperfusion on intestinal absorption and permeability during cardiopulmonary bypass Gastroenterology 1994; 106: 318-323.

18. Johnston J.D., Harvey C.J., Menzies LS., and Treacher D.F. Gastrointestinal permeability and absorptive capacity in sepsis. Crit Cane Med 1996, 24: 1144-1149.

19. Murray M.J., Gonze M.D., Nowak L.R., and Cobb C.F. Serum D(-) -lactate levels as an aid to diagnosing acute intestinal ischemia. Am J Surg 1994; 167: 575-578.

20. Brandt R.B., Siegel S.A., Waters M.G., and Bloch M.F. Spectrophotometric assay for D-(-)lactate in plasma. Anal Biochem 1980, 102: 3946.

21. Ford R.P.K., Menzies L.S., Phillips A.D., Walker-Smith J.A., and Turner M.W. Intestinal sugar permeability: relationship to diarrhoeal disease and small bowel morphology. J Pediatr Gastroenterol Nutr $1985 ; 4: 568-574$. 
22. Van der Hulst R.R., van Kreel B.K., von Meyenfeldt M.F., Brummer R.J., Arends J.W., Deutz N.E., and Soeters P.B. Glutamine and the preservation of gut integrity. Lancet 1993; 334: 1363-1365.

23. Buchman A.L., Moukarzel A.A., Bhuta S., Belle M., Ament M.E., Eckhert C.D., Hollander D., Gornbein J., Kopple J.D., and Vijayaroghavan S.R. Parenteral nutrition is associated with intestinal morphologic and functional changes in humans. J Parenter Enter Nutr 1995; 19: 453-460.

24. Hollander D. The intestinal permeability barrier. A hypothesis as to its regulation and involvement in Crohn's disease. Scand J Gastmenterol 1992; 27: 721-726.

25. Madara J.L. Loosening tight junctions; lessons from the intestine. J Clin Invest 1989; 83: 10891094.

26. McKay D.M. and Baird A.W. Cytokine regulation of epithelial permeability and ion transport. Gut 1999; 44: 283-289.

27. O'Boyle C.J., MacFie J., Dave K., Sagar P.S., Poon P., and Mitchell C.J. Alterations in intestinal barrier function do not predispose to translocation of enteric bacteria in gastroenterological patients. Nutrition $1998 ; 14: 358-362$.

28. Kanwar S., Windsor A.C., Welsh F., Barclay G.R., Guillou P.J., and Reynolds J.V. Lack of correlation between failure of gut barrier function and septic complications after major upper gastrointestinal surgery. Ann Sung 2000; 231: 88-95.

29. Davin J.C., Forget P., and Mahieu P.R. Increased intestinal permeability to ${ }^{51} \mathrm{Cr}$-EDTA is correlated with IgA immune complex-plasma levels in children with IgA-associated nephropathies. Acta Paediatr Scand 1988; 77: 118-124.

30. Ramage J.K., Stanisz A., Scicchitano R., Hunt R.H., and Perdue M.H. Effects of immunologic reactions on rat intestinal epithelium. Correlation of increased intestinal permeability to ${ }^{51} \mathrm{Cr}$-EDTA and ovalbumin during acute inflammation and anaphylaxis. Gastroenterology 1988; 94: 1368-1375.

31. Ferry D.M., Butt T.J., Broom M.F., Hunter J., and Chadwick V.S. Bacterial chemotactic oligopeptides and the intestinal mucosal barrier. Gastroenterology 1989; 97:61-67.

32. Gewolb I.H., Schwalbe R.S., Taciak V.L., Harrison T.S., and Panigrahi P. Stool microflora in extremely low birthweight infants. Anch Dis Child Fetal Neonatal Ed 1999; 80: F167-F173.

33. Craig R.M. and Atkinson A.J. D-xylose testing: a review. Gastroenterology 1988; 95: 223-231.

34. Fordtran J.S., Clodi P.H., Soergel K.H., and Ingelfinger F.J. Sugar absorption tests, with special reference to 3-0-methyl-D-glucose and D-xylose. Ann Intern Med 1962; 57: 883-891. 


\section{CHAPTER 4}

Early Minimal Enteral Feeding

in Very Low Birth Weight Neonates:

an Effective Strategy to Improve Mucosal Functions of the Immature Gut 


\section{Abstract}

Objective. Functional immaturity of the gut poses a considerable problem in the nutritional management of preterm neonates, and plays a role in the pathophysiology of gastrointestinal complications in these patients. Previous reports have shown that early introduction of enteral feeding, in conjunction with parenteral nutrition, may be beneficial to these patients. We investigated to what extent the initiation of minimal enteral feeding shortly after birth, in comparison with exclusive parenteral feeding, provides an effective intervention to promote the maturation of intestinal barrier function and sugar absorptive capacity in preterm neonates during the early postnatal period.

Study design. Preterm neonates ( $25-32$ weeks gestation, $n$-120) stratified by gestational age were randomly assigned to start minimal enteral feeding ( $12 \mathrm{ml} /$ day) either at day 1 (intervention group, $n-61$ ) or at day 8 (control group, $n$-59) after birth. A sugar permeability-absorption test was performed at 1, 4, 7, and 14 days after birth. The lactulose/L-rhamnose ratio was determined as a marker of intestinal permeability. Urinary excretion percentages of $\mathrm{D}$-xylose and 3-0-methyl-D-glucose were determined as markers of passive and active carrier-mediated monosaccharide absorption, respectively.

Results. Intestinal permeability during the first week after birth was significantly lower in the intervention as compared with the control group. This effect was due to a preservation of epithelial integrity in the intervention group. Active carrier-mediated monosaccharide absorption at the end of the second postnatal week was significantly higher in the intervention as compared with the control group.

Conclusions. The introduction of low volumes of enteral feeding shortly after birth is an effective method to improve the barrier function as well as to enhance the maturation of the sugar absorptive capacity of the gut in very low birth weight neonates. 


\section{INTRODUCTION}

Functional immaturity of the intestinal tract predisposes preterm neonates to intestinal complications, such as feeding intolerance and necrotising enterocolitis, and poses a considerable problem in the nutritional management of these patients. Due to potential complications caused by the introduction of enteral feeding, it is common practice to provide total parenteral nutrition as the sole mode of nutritional support during the initial postnatal period. However, studies in adults have shown that withholding enteral feeding may rapidly lead to structural and functional deterioration of the gut, including a decrease in mucosal barrier function (1-4). Moreover, we recently reported that the barrier function and the sugar absorptive capacity of the intestinal epithelium are low during the early postnatal period in parenterally fed preterm neonates (5).

Early administration of small volumes of enteral feeding in addition to parenteral nutrition has been shown to exert beneficial effects on the premature gastrointestinal tract, including enhanced intestinal motility, increased lactase activity, and greater mineral absorption (6-9). Furthermore, early minimal enteral feeding has been reported to improve feeding tolerance and shorten the duration of hospital stay (10-16).

The primary objective of this study was to identify to what extent enteral nutrients can be used to enhance the maturation of the epithelial barrier function and absorptive capacity of the immature intestine. In a randomised controlled trial we evaluated intestinal permeability and carrier-mediated monosaccharide absorption in preterm neonates receiving early minimal enteral feeding compared with those receiving exclusively parenteral nutrition during the initial postnatal period.

\section{METHODS}

\section{Patients and study design}

The randomised controlled trial was conducted in the neonatal intensive care unit of the University Hospital Maastricht, and was approved by the local medical ethics committee. Parents of eligible neonates were informed in detail about the study, and written informed consent was obtained before enrollment.

Preterm neonates admitted to the intensive care unit were eligible for the study if they were born at a gestational age of 25-32 weeks and if postnatal age was less than 24 hours. Criteria for exclusion included: major congenital anomalies, severe asphyxia (defined as 5-minute Apgar score $\leq 3$ ), persistent hypoxaemia or respiratory acidosis, and severe hypotension. At study entry, for each neonate we recorded gestational age, birth weight, sex, Apgar score at 1 and 5 minutes, CRIB score (Clinical Risk Index for Babies), presence of asphyxia, mode of delivery, prolonged rupture of the membranes (>24 h), (pre)eclampsia/HELLP syndrome, chorioamnionitis, antenatal administration of corticosteroids, tocolysis, and multiple pregnancy.

Neonates enrolled in the study were stratified by gestational age $\left(25-27^{\circ}, 28\right.$ $29^{* 6}, 30-32$ weeks). A randomisation list was generated by a computer program for 
each gestational age category, ensuring balance of group size by employing block sizes of 4. At the day of birth, infants were assigned to either the early minimal enteral feeding (intervention) group or the late enteral feeding (control) group. From day 1 through 7 after birth, the intervention group received $12 \mathrm{ml} /$ day of enteral feeding, and the remaining fluids as standard parenteral nutrition solutions, whereas the control group received only parenteral nutrition. Enteral feeding was administered as 2-ml bolus feedings via a nasogastric tube at 4-hour intervals (9), and consisted of either human milk (obtained and used within $\mathbf{4 8}$ hours) or full strength preterm formula (Nenatal, Nutricia, Zoetermeer, the Netherlands), according to the choice of the parents. The regimen of parenteral nutrition, as well as the rate of increase and total fluid intake were the same for both groups. Total fluid intake was initially $80 \mathrm{ml} / \mathrm{kg} /$ day and was increased by $20 \mathrm{ml} / \mathrm{kg}$ /day up to approximately $150 \mathrm{ml} / \mathrm{kg} /$ day. Care was taken to adjust the volume of parenteral nutrition for enteral fluid intake, in order to provide comparable fluid, energy, and nutrient intakes among intervention and control groups.

At day 8 after birth, enteral feeding was also initiated in the control group. In both groups the enteral feeding volume was gradually increased, while the volume of parenteral nutrition was decreased, until complete enteral nutrition (approximately $150 \mathrm{ml} / \mathrm{kg} /$ day) was achieved. The rate of enteral feeding increment was determined by the attending neonatologist, and adapted as required by the infants' clinical condition. In case of feeding intolerance, as defined by emesis, frequent gastric residuals of more than $3 \mathrm{ml}$, abdominal distension, and/or ileus, enteral feedings were reduced or withheld until the problem resolved. Nonbloody gastric residuals of less than $3 \mathrm{ml}$ per 2 hours were refed.

\section{Assessment of intestinal permeability and monosaccharide absorption}

To allow the simultaneous evaluation of intestinal permeability and monosaccharide absorption, we conducted a sugar permeability-absorption test at day 1, 4, 7, and 14 after birth. This test is noninvasive, requires only minimal handling, and can be used safely in preterm neonates (17). The sugar solution was produced in the hospital pharmacy and consisted of $8.6 \mathrm{~g}$ lactulose (Centrafarm, Etten-Leur, the Netherlands), $140 \mathrm{mg}$ L-rhamnose (Acros Organics, Pittsburgh, PA), $70 \mathrm{mg}$ D-xylose (Genfarma, Maarssen, the Netherlands), and $140 \mathrm{mg}$ 3-O-methyl-D-glucose (Sigma, St. Louis, MO) dissolved in $100 \mathrm{ml}$ demineralised water ( $425 \mathrm{mOsm} / \mathrm{l})$. Each test day, $2 \mathrm{ml}$ of the sugar solution was administered to the neonate via the nasogastric tube. All urine voided over the next 4 hours was collected in an adhesive urine bag (Urinocol Premature, Braun Biotrol, Paris, France). In the intervention group, no enteral feeding was given in the 4 hours preceding and following ingestion of the test solution at day 1,4 , and 7 . Thus, these infants received $5 \times 2 \mathrm{ml}$ of minimal enteral feeding and $2 \mathrm{ml}$ of the sugar solution at the test days. At day 14, oral feeding was withheld for 2 hours prior to and after the administration of the sugar solution in both the intervention and the control groups. These time points were chosen to minimise interference with routine nursery protocols. The complete 4-hour urine volume was measured, and a $2 \mathrm{ml}$ aliquot was stored at $-80{ }^{\circ} \mathrm{C}$ until analysis. The sugar absorption test critically relies on a complete urine collection. Therefore, in case urine collection failed in an infant, the test was repeated the next day. If 
urine sampling failed again, then measurements for the infant at this particular test day were regarded as missing values. Urinary concentrations of lactulose, L-rhamnose, D-xylose, and 3-O-methyl-D-glucose were determined by gas-liquid chromatography as previously described (18).

The four saccharides used in this test cross the intestinal epithelium via different pathways and are cleared by the kidneys. The percentage of the orally administered dose of each saccharide which is excreted in the urine thus reflects the functional state of the particular intestinal permeation pathway. Lactulose is a disaccharide which crosses the intestinal epithelium by passive diffusion through paracellular tight junctions. L-rhamnose is a monosaccharide which crosses the epithelium mainly by transcellular passive diffusion through aqueous pores. The urinary excretion percentages of lactulose and rhamnose are markers for paracellular and transcellular diffusion, respectively. To correct for nonmucosal factors which may affect intestinal uptake of these saccharides, including the rate of gastric emptying, intestinal transit time, and renal clearance, the urinary excretion percentages of lactulose and rhamnose were expressed as the lactulose/rhamnose excretion ratio ( $\mathrm{L} / \mathrm{R}$ ratio). Since nonmucosal factors will affect urinary excretion of both saccharides to a similar extent, the $\mathrm{L} / \mathrm{R}$ ratio provides a reliable index of the permeability of the intestinal epithelium $(19,20)$. D-xylose and 3-O-methyl-Dglucose are monosaccharides which are absorbed by intestinal epithelial cells via passive and active carrier-mediated transport mechanisms, respectively. The urinary recovery of orally administered xylose and methylglucose are markers for passive and active carrier-mediated monosaccharide absorption, respectively $(21,22)$.

\section{Clinical outcome variables}

Clinical outcome variables were evaluated at 14 and 30 days of age, or at hospital discharge, whichever occurred first. These include the number of days required to reach full enteral nutrition $(150 \mathrm{ml} / \mathrm{kg} /$ day), the net enteral balance (enteral intake - gastric residual) at 14 and 30 days, the number of days that oral feedings were withheld, body weight gain, the periods of sepsis (defined as clinical signs and positive blood culture), and necrotising enterocolitis (NEC). Diagnosis of NEC was based on the presence of clinical signs, such as abdominal distension and rectal blood loss, and the presence of intestinal pneumatosis on abdominal radiographs. In addition, in the course of the trial we determined short-term growth in a subset of infants ( $n-20$ in both intervention and control groups), by measuring frontooccipital circumference, crown-rump length, and lower leg length, using methods published previously (23).

Several measures were taken to ensure unbiased evaluation of trial outcome. The principal investigator (ER) was by no means involved in the clinical management of the neonates. The staff of the neonatal intensive care unit, who were responsible for daily care of the infants, were not involved in the trial. Urine samples were coded, and chemical analysis was performed by the department of clinical chemistry. 


\section{Statistical methods}

Data of the sugar absorption test are presented as $95 \%$ confidence intervals (CI) for mean differences between intervention and control groups in the text, and as mean \pm SEM in the graphs. Clinical variables are presented as median and interquartile range. Data analysis was by intention to treat, i.e., all randomised patients were included. The overall effects in time of the indices of intestinal permeability and absorptive capacity were determined by a repeated measurements analysis, using the GLM procedure in SPSS version 10.

The effects of early minimal enteral feeding were analysed with generalised least squares (GLS) random effects models, using Stata Statistical Software version 7. These models take into account the fact that each neonate had up to three follow-up moments (repeated measures). In addition, they handle missing values flexibly and provide an overall significance test of the treatment effect, thus avoiding the multiple testing problems that occur with multiple endpoints (potential effects on day 4, 7, and 14). In all these (longitudinal) analyses, the corresponding values at randomisation were used as a co-variable. The time points were modelled as indicator variables (day 4 as reference). In each longitudinal analysis we attempted to answer the question whether the effects of early minimal enteral feeding differed between time point and gestational age (in three categories). When no such interactions emerged, the model was simplified to answer the question whether the effects of early minimal enteral feeding differed between the time points (treatment-time interactions). If this was not the case, a simple model estimating the overall treatment effect was adopted. The question to what extent the effects of early minimal enteral feeding differed between human milk and preterm formula was analysed using conventional least squares regression methods controlling for gestational age category, CRIB score, and baseline value. Clinical (dichotomous) variables were compared using the chi-squared test or MannWhitney $\mathrm{U}$ test. To assess interactions, a cut-off level of $\mathrm{p}=0.10$ was used. In all other cases statistical significance was defined as $\mathrm{p}<0.05$. 


\section{RESULTS}

Recruitment of patients started in July 1997 and ended in September 1999. We recruited 120 preterm neonates born between 25 and 32 weeks gestation. Neonates were stratified for gestational age: $25-27^{+6}(n-37), 28-29^{* 6}(n-48)$, and $30-32$ weeks gestation $(n-35)$. Neonates in each of these categories were randomly allocated to the early minimal enteral feeding (intervention) group or the late enteral feeding (control) group within 24 hours of birth. Clinical characteristics of the patients are summarised in Table 1. The sugar permeability-absorption test was not successful in all neonates at all four time points, due to one of the following reasons: failure of complete urine collection, failure of chemical analysis, unstable clinical condition, or transfer to another hospital.

Table 1. Clinical characteristic of the patients at study entry ${ }^{*}$

\begin{tabular}{|c|c|c|c|c|c|c|}
\hline \multirow[b]{2}{*}{ Variable } & \multicolumn{2}{|c|}{ Neonates $25 \cdot 27^{+6} \mathrm{wk}$} & \multicolumn{2}{|c|}{ Neonates $28 \cdot 29^{+6} \mathrm{wk}$} & \multicolumn{2}{|c|}{ Neonates $30-32 \mathrm{wk}$} \\
\hline & $\begin{array}{l}\text { Intervention } \\
n-19\end{array}$ & $\begin{array}{l}\text { Control } \\
n-18\end{array}$ & $\begin{array}{l}\text { Intervention } \\
n-24\end{array}$ & $\begin{array}{l}\text { Control } \\
n=24\end{array}$ & $\begin{array}{l}\text { Intervention } \\
n-18\end{array}$ & $\begin{array}{l}\text { Control } \\
n=17\end{array}$ \\
\hline Birth weight (g) & 840 & 880 & 1140 & 1050 & 1368 & 1340 \\
\hline Femalemale ( $\mathbf{S}$ female) & $415(21.1)$ & $126(66.7)$ & $8.16(333)$ & $11: 13(458)$ & $5: 13(27.8)$ & $8.9(4.1)$ \\
\hline \multicolumn{7}{|l|}{ Apgar score } \\
\hline $1 \mathrm{~min}$ & $5(48)$ & $6(3.58)$ & $7(48)$ & $7(5-9)$ & $7.5(4.75-9)$ & $5(48)$ \\
\hline $5 \mathrm{~min}$ & $8(7-9)$ & $9(7-9)$ & $9(89)$ & $9(89)$ & $8(8.9 .25)$ & $9(75-10)$ \\
\hline CRIB score & $4(1-6)$ & $1.5(1-7)$ & $1(1-3)$ & $1.5(1.5)$ & $1(0-2)$ & $1(0.5-1.5)$ \\
\hline Asphyxia (X) & 15.8 & 16.7 & 20.8 & 16.7 & 16.7 & 176 \\
\hline Caesarean delivery $(\mathbf{(})$ & 263 & 38.9 & 41.7 & 625 & 66.7 & 58.8 \\
\hline Ruptured membranes $>24 \mathrm{~h}(\%)$ & 36.8 & 22.2 & 50 & 29.2 & 278 & 35.3 \\
\hline Chorioamnionitis (\$) & 31.6 & 16.7 & 12.5 & 4.2 & 0 & 0 \\
\hline (Pre)eclampsia/HELP (\$) & 5.3 & 5.6 & 83 & 16.7 & 11.1. & 5.9 \\
\hline Antenatal corticosteroids (\%) & 68.4 & 833 & 66.7 & 54.2 & 61.1 & 47.1 \\
\hline Tocolysis (\$) & 68.4 & 72.2 & 62.5 & 37.5 & 44.4 & 52.9 \\
\hline Multiple prognancy (\$) & 36.8 & 278 & 37.5 & 25 & 44.4 & 353 \\
\hline
\end{tabular}

"Values are percentage of patients except for birth weight, Apgar score, and CRIB score, which are median (interquartile range) 


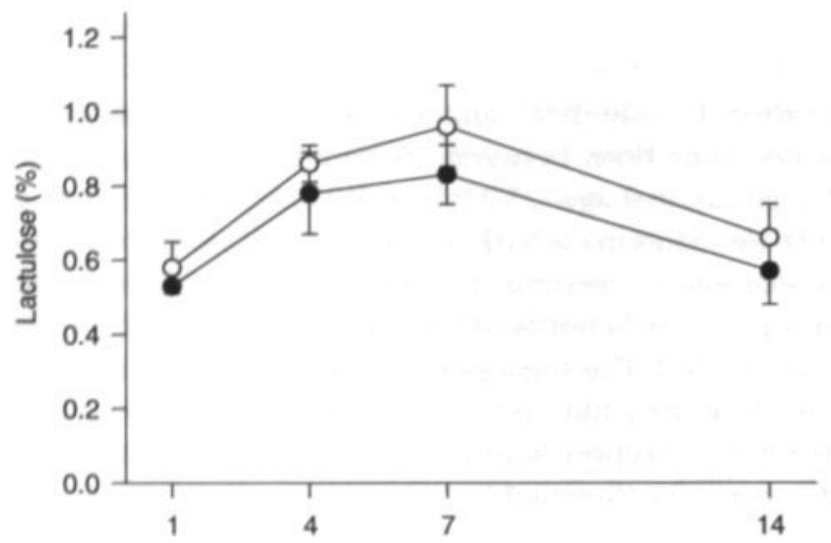

- control

intervention

\section{Figure 1A}

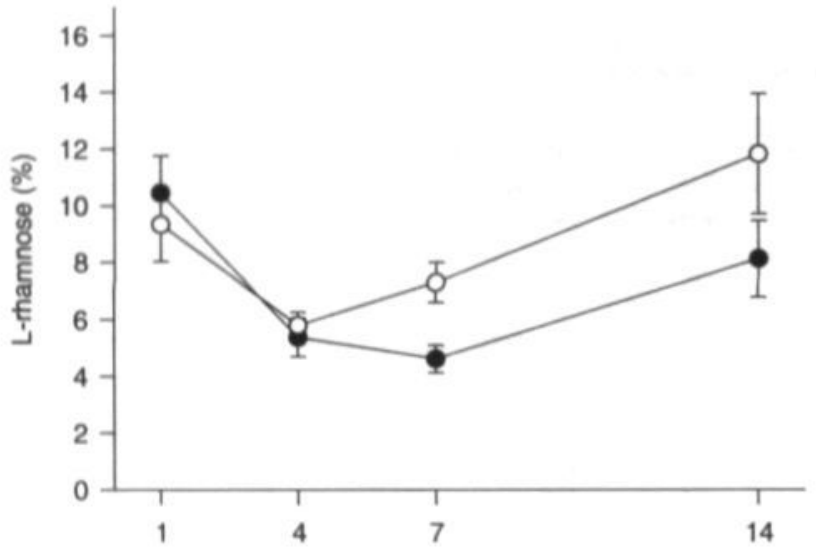

Figure 1B

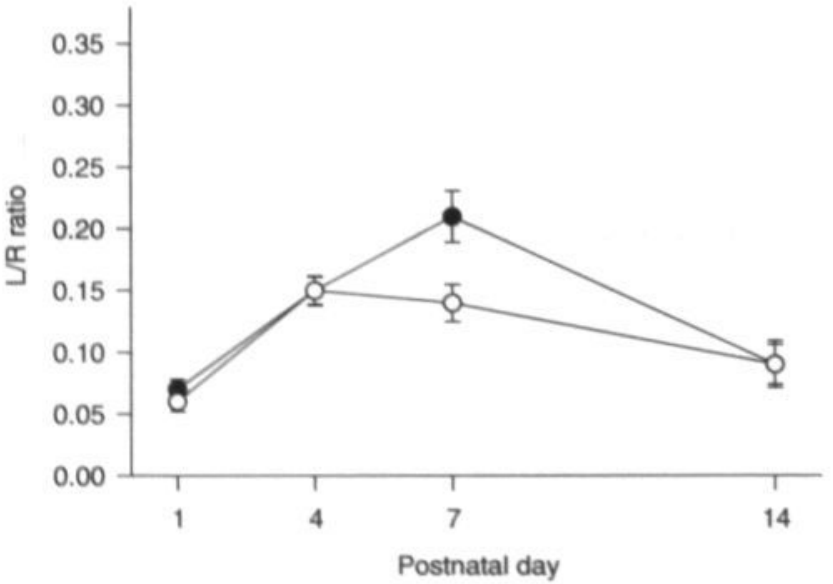

Figure 1C

\section{Figure 1}

Effect of early minimal enteral feeding on intestinal paracellular diffusion (A), transcellular diffusion (B), and permeability $(\mathrm{C})$, as represented by the urinary excretion percentages of orally administered lactulose, L-rhamnose, and the lactulose/rhamnose excretion ratio, during the first two weeks after birth in preterm neonates of 25-32 weeks gestation. Data are mean \pm SEM. 
Effect of early minimal enteral feeding on intestinal permeability

Lactulose excretion increased during the first week after birth in all neonates (quadratic association, $\mathrm{p}=0.001$ ). The difference in lactulose excretion between the intervention and the control group during the period of observation was $-0.024 \%$ (95\% CI -0.18 to $0.13 \%, \mathrm{p}=0.767$; Fig. 1A).

Repeated measurements analysis of L-thamnose excretion showed a significant decrease during the first week after birth in all neonates (quadratic association, $\mathrm{p}-0.01$ ). However, overall rhamnose excretion during the period of observation was higher $(2.1 \%, \mathrm{Cl} 0.5$ to $3.6 \%)$ in the intervention group compared with the control group ( $\mathrm{p}-0.009$, Fig. 1B). We found no indications that this effect depended on gestational age.

As a result of these changes in lactulose and mamnose recovery, the $\mathrm{L} / \mathrm{R}$ ratio for all neonates (intervention and control groups) significantly increased during the first week after birth (quadratic association, $\mathrm{p}=0.000$ ). The $\mathrm{L} / \mathrm{R}$ ratio at day 7 after birth was lower $(-8.3 \%, \mathrm{CI}-13.1$ to $-3.5 \%)$ in the intervention group as compared with the control group ( $p=0.001$, Fig. 1C). We found no indication that this effect depended on gestational age.

\section{Effect of early minimal enteral feeding on monosaccharide absorption}

Repeated measurements analysis demonstrated significant increases in the excretion percentages of D-xylose and 3-O-methyl-D-glucose over time for the group of neonates as a whole (linear associations, p -0.000 and p -0.000 , respectively). During the period of observation, xylose recovery was not significantly different between the intervention and control groups (1.5\%, $\mathrm{CI}-0.7$ to $3.7 \%$; Fig. 2A). Methylglucose excretion at day 14 after birth was higher $(4.0 \%$, CI 1.2 to $6.9 \%)$ in the intervention as compared with the control group ( $\mathrm{p}-0.007$, Fig. 2B). We found no indications that this effect depended on gestational age.

\section{Effect of early minimal enteral feeding with human milk versus preterm formula}

Within the intervention group 19 infants received human milk and 42 infants received preterm formula feeding during the first week after birth. The difference in $L / R$ ratio at day 7 between human milk and formula fed neonates, adjusted for gestational age category, CRIB score, and baseline value, was $4.1 \%$ (CI -0.2 to $9.8 \%, \mathrm{p}=0.15)$. Similarly, the adjusted difference in urinary excretion percentages of methylglucose at day 14 between the two groups was $-2.3 \%$ ( $\mathrm{CI}-2.8$ to $-7.4 \%$, $\mathrm{p}-0.367)$. In addition, there were no differences between human milk $(n-39)$ and formula fed ( $n=41)$ neonates (intervention and control groups) in intestinal permeability and monosaccharide absorption at day 14 , or in clinical outcome. 


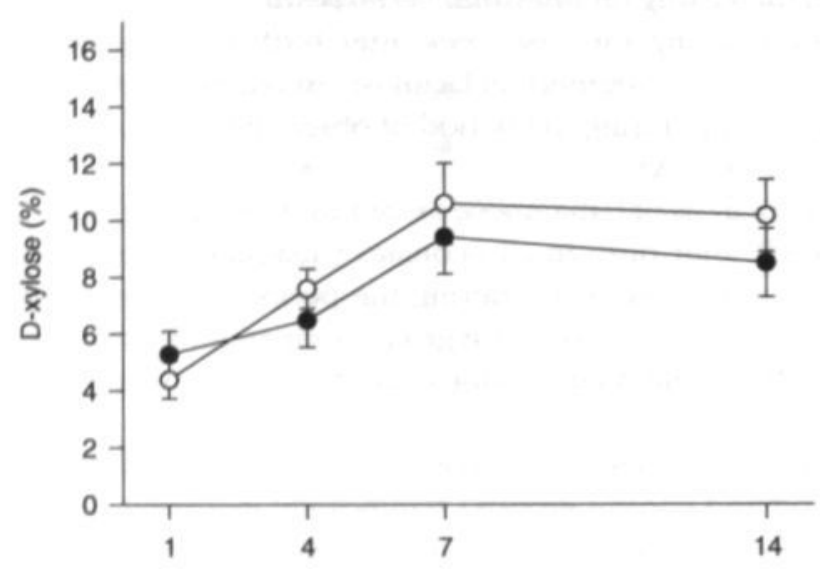

- control

intervention

Figure 2A

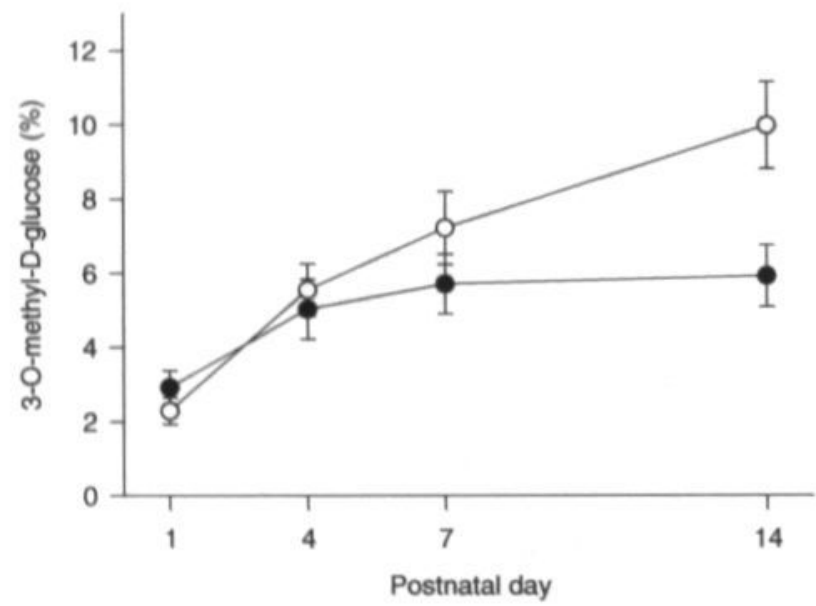

Figure 2B

\section{Figure 2}

Effect of early minimal enteral feeding on intestinal passive (A) and active (B) carrier-mediated monosaccharide absorption, as determined by the urinary excretion percentages of orally administered D-xylose and 3-0-methyl-D-glucose, during the first two weeks after birth in preterm neonates of 25-32 weeks gestation. Data are mean \pm SEM. 
Clinical outcome variables are summarised in Table 2 . In neonates younger than 28 weeks gestation, the net enteral balance at day 14 was significantly higher in the intervention as compared with the control group $(\mathrm{p}-0.020)$. A similar tendency was found in neonates born at $28.29^{\circ} 6$ weeks $(\mathrm{p}-0.063)$. For the study population as a whole, no significant differences were noted between intervention and control neonates with regard to the number of days to full enteral feeding, the number of days that enteral feeding was withheld, and the incidence of sepsis. Eight cases of necrotising enterocolitis were diagnosed in the course of the trial, which were evenly distributed between the intervention and the control group. None of the neonates with necrotising enterocolitis died.

Five bables died during the study period. Two infants suffered from moderate respiratory distress syndrome, and died at day 4 after birth because of extensive cerebral intraparenchymal haemorrhage. In three cases mortality was related to infectious complications. One of these neonates was colonised with Eschericha coli from birth onward and died following the development of systemic infection at day 11 of life. The other two infants died at day 10 and 16 after birth as a consequence of Candida albicans and Enterobacter cloacae sepsis, respectively. All babies who died were born at less than 29 weeks gestation, after multiple preg: nancy, had a CRIB score $>2$, and received early minimal enteral feeding with human milk or preterm formula. The difference in mortality rate between the intervention and control group was $8.2 \%$ (CI 1.3 to $15.1 \%, \mathrm{p}=0.058$ ).

Lower leg length gain at day 14 and day 30 was significantly greater in the intervention as compared with the control group as a whole $(4.6 \pm 0.39$ vs. $3.5 \pm 0.37 \mathrm{~mm}$, $\mathrm{p}=0.046$ and $12.2 \pm 1.05$ vs. $9.1 \pm 0.93 \mathrm{~mm}, \mathrm{p}=0.023$ ). A similar difference was found in the increase in crown-rump length at 30 days of age $(36.1 \pm 2.20 \mathrm{vs}$. $25.3 \pm 3.23$ $\mathrm{mm}, \mathrm{p}=0.022)$.

Table 2. Clinical outcome ${ }^{2}$

\begin{tabular}{|c|c|c|c|c|c|c|}
\hline Variable & $\begin{array}{l}\text { Neonates } 25-2 \\
\text { Intervention } \\
n=19\end{array}$ & $\begin{array}{l}27^{+6} \text { wk } \\
\text { Control } \\
n=18\end{array}$ & $\begin{array}{l}\text { Neonates } 28-2 \\
\text { Intervention } \\
n-24\end{array}$ & $\begin{array}{l}29^{+6} \text { wk } \\
\text { Control } \\
n-24\end{array}$ & $\begin{array}{l}\text { Neonates } 30-3 \\
\text { Intervention } \\
n=18\end{array}$ & $\begin{array}{l}32 \text { wk } \\
\text { Control } \\
n=17\end{array}$ \\
\hline Age full enteral feeding (days) & $20(17-22)$ & $20(1826)$ & $16(15-26)$ & $20(16-23)$ & $19(15-27)$ & $14(13-17)$ \\
\hline \multicolumn{7}{|l|}{ Net enteral balance (ml) } \\
\hline 14 days & $82(35-101)^{*}$ & $30(-1-17)$ & $101(29137)$ & $26(583)$ & $83(4-151)$ & $137(101-173)$ \\
\hline 30 days & $195(149216)$ & $149(105-199)$ & $193(-1-271)$ & $203(124-242)$ & $259(67-321)$ & $238(167.266)$ \\
\hline Enteral feeding withheld (days) & $0(0-1)$ & $0(0-2)$ & $0(0-1)$ & $0(0-2)$ & $0(0-1)$ & $0(0-0)$ \\
\hline \multicolumn{7}{|l|}{ Weight gain $(g)$} \\
\hline 14 days & $73(13-108)$ & $35(-894)$ & $\cos (25-143)$ & $100(50-190)$ & $155(66-248)$ & $102(76-158)$ \\
\hline 30 days & $310(250-420)$ & $275(159371)$ & $433(370675)$ & $550(340555)$ & $580(365-750)$ & $375(283-498)$ \\
\hline Sepsis, no. of neonates ( $(\mathbf{S})$ & $6(31.6)$ & $10(55.6)$ & $12(50)$ & $11(45.8)$ & $9(50)$ & $4(23.5)$ \\
\hline Necrotising enterocolitis, na ( $(\mathbf{X})$ & $2(10.5)$ & $1(5.6)$ & $2(8.3)$ & $2(8.3)$ & $1(56)$ & $0(0)$ \\
\hline Deaths, no. (\$) & $3(15.8)$ & $0(0)$ & $2(8.3)$ & $0(0)$ & $0(0)$ & $0(0)$ \\
\hline
\end{tabular}

a Values are either median (interquartile range) or number (\%) of patients

- Differs significantly from control group, p<0.05 


\section{DISCUSSION}

The aim of this randomised controlled trial was to investigate the effect of early minimal enteral feeding on the postnatal development of gut mucosal functions in preterm neonates during the initial postnatal period. The data show that the introduction of low volumes of enteral feeding starting shortly after birth in conjunction with parenteral nutrition, in comparison with exclusive parenteral feeding, improved the barrier function and enhanced the sugar absorptive capacity of the intestinal epithelium in very low birth weight neonates.

Our data confirm the role of enteral feeding in the maintenance of the structural integrity of the intestinal epithelial barrier. The common practice to provide exclusively parenteral nutrition initially after birth was associated with a rise in intestinal permeability, indicating that the barrier function of the intestinal epithelium deteriorated in the absence of luminal nutrients $(3,24)$. This increase was the combined result of loosening of intercellular tight junctions, as indicated by the increase in lactulose recovery, as well as loss of functional mucosal surface area, as indicated by the reduction in rhamnose absorption. By comparison, neonates which received small amounts of enteral feeding in addition to the parenteral nutrition from the first day after birth onward had a significantly better absorption of rhamnose. This indicates that the initiation of minimal enteral feeding shortly after birth reduced the loss of functional intestinal epithelial cells, thereby preserving mucosal integrity of the immature gut in the early postnatal period.

Although early enteral feeding improved the barrier function of the intestinal epithelium, it did not provide evidence for a lower incidence of septic complications. However, the integrity of the epithelium is only one part of the intestinal barrier. Other factors, including mucus secretion, peristalsis, gut immune function, and splanchnic blood flow are also involved in the maintenance of gut barrier function.

Early minimal enteral feeding also stimulated the maturation of active carriermediated monosaccharide absorption in the intestine of preterm neonates, as indicated by the increase in the recovery of 3-O-methyl-D-glucose. Transport of this monosaccharide is similar to that of glucose and galactose, i.e., depends on an active $\mathrm{Na}^{+} / \mathrm{K}^{+}$-ATPase at the basolateral membrane of the enterocyte, and is mediated via the same carrier proteins, $\mathrm{Na}^{+} /$glucose cotransporter (SGLT1) at the brush border membrane, and facilitated glucose transporter (GLUT2) at the basolateral membrane (25).

The enhancement of methylglucose absorption in the early minimal feeding group was not accompanied by an increase in the absorption of other test saccharides. This strongly suggests that the effect of early minimal enteral feeding was specific for the active carrier-mediated transport mechanisms of the intestinal epithelium, and was not solely due to an increase in mucosal surface area, increased paracellular diffusion, or extraintestinal factors. A potential mechanism for the enhanced uptake of methylglucose may be increased expression and activity of the epithelial carrier proteins as a result of the presence of sugars in the gut lumen $(25,26)$. This idea is supported by findings in neonatal animal studies which showed that the expression of the brush-border membrane transporter proteins for 
glucose (SGLT1) and fructose (GLUTS) is enhanced by the supply of glucose and fructose, respectively (27-29). Irrespective of the underlying mechanisms, improvement in active monosaccharide transport may lead to better uptake of orally administered milk or formula feeding, and support the nutritional status and growth of the preterm infant during the early postnatal period.

In contrast to previous studies $(9,30,31)$, the current study did not show additional benefit of human milk over cow's milk-based preterm formula with regard to either intestinal epithelial barrier function and absorptive capacity or clinical outcome. This suggests that the beneficial effects of early enteral feeding on preservation of epithelial integrity and on stimulation of carrier-mediated monsaccharide absorption are related to the presence of nutrients in the intestinal lumen (32), rather than on the presence of growth factors or other bioactive agents found in human milk. Alternatively, the period of human milk administration may have been too brief to identify measurable benefits of breast milk feeding over formula feeding on gut mucosal functions.

Short-term growth rates were significantly higher in neonates receiving early minimal enteral feeding compared with those receiving only parenteral feeding. These data are consistent with previous observations showing that minimal enteral feeding is associated with greater absorption and retention of minerals such as calcium and phosphorus, as well as elevated levels of serum calcium and alkaline phosphatase activity, as indicators of postnatal growth (9).

Contrary to a number of previous reports, there were no differences among groups with regard to nutritional milestones, including the time to attain complete tube-feeding, and thus duration of parenteral nutrition, or the number of days that feedings were withheld. However, both in the intervention and the control group full enteral nutrition was already established by approximately 20 days of age, which is considerably earlier than in previous studies in neonates of similar gestational age $(6,9-11,13-16)$. Furthermore, the overall incidence of NEC was lower $(6.7 \%)$ than that reported by others (about $12 \%)(9,16)$. These improvements in clinical outcome in the current trial may be due to the overall earlier introduction and rapid progression of enteral feeding. Our feeding strategy differed from previous reports in that enteral feeding was considered early when initiated by 24 hours after birth, and late when administered after the first week of life. In contrast, in most other reports the introduction of 'early' feeding was delayed until 3 to 8 days after birth, whereas late feeding was associated with a period of enteral starvation as long as two weeks.

The mortality rate was higher in the intervention as compared with the control group. At first sight, it may be suggested that this was related to the intervention itself. However, the causes of death did not appear to be of intestinal origin. Furthermore, the rate of intestinal complications, such as necrotising enterocolitis, was similar in both groups. Therefore, it is not very likely that the higher mortality rate in the intervention groups can be attributed to the early enteral feeding.

In conclusion, these data confirm and expand on previous observations on the beneficial effect of early minimal enteral feeding on the maturation of intestinal functioning (6-9). The practice of initiating low volume enteral feedings directly after birth offers an effective strategy to support the intestinal adaptation to 
extrauterine life in preterm neonates by preventing the loss of mucosal integrity and inducing a precocious maturation of intestinal nutrient transport.

\section{ACKNOWLEDGMENTS}

We thank all the families for their participation in this study, and the staff of the neonatal intensive care unit, Mr. B. Panis, Mrs. A. Gerver, and the department of Clinical Chemistry for their assistance. 


\section{References}

1. Van der Hulst R.R.W.J., von Meyenfeldt M.F., van Kreel B., Brummer R.J.M., Arends J.W., Deutz N.E.P., and Soeters P.B. Glutamine and the preservation of gut integrity. Lancet 1993; 334: 1363-1365.

2. Maxton D.G., Menzies L.S., Slavin B., and Thompson R.P.H. Small-intestinal function during enteral feeding and starvation in man. Clin Sci 1989, 77: 401-406.

3. Buchman A.L., Moukarzel A.A., Bhuta S., Belle M., Ament M.E., Eckhert C.D., Hollander D., Gornbein J., Kopple J.D., and Vijayaroghavan S.R. Parenteral nutrition is associated with intestinal morphologic and functional changes in humans. J Panenter Enter Nutr 1995; 19:453-460.

4. Van der Hulst R.R.W.J., von Meyenfeldt M.F., van Kreel B.K., Thunnissen F.B.J.M., Brummer R.J.M., Arends J.W., and Soeters P.B. Gut permeability, intestinal morphology, and nutritional depletion. Nutrition 1998; 14: 16.

5. Rouwet E.V., Heineman E., Buurman W.A., Ter Riet G., Ramsay G., and Blanco C.E. Early postnatal development of intestinal barrier function and absorptive capacity in preterm neonates. Pediatric Research, in press.

6. Berseth C.L. and Nordyke C. Enteral nutrients promote postnatal maturation of intestinal motor activity in preterm infants. Am J Physiol 1993; 264: G1046-G1051.

7. MoClure R.J. and Newell S.J. Randomised controlled trial of trophic feeding and gut motility. Anch Dis Child Fetal Neonatal Ed 1999, 80: F54-F58.

8. Shulman R.J., Schanler R.J., Lau C., Heitkemper M., Ou C.-N., and O'Brian Smith E. Early feeding, feeding intolerance, and lactase activity in preterm infants. J Pediatr 1998; 133: 645-649.

9. Schanler R.J., Shulman R.J., Lau C., O'Brian Smith E., and Heitkemper M.M. Feeding strategies for premature infants: randomized trial of gastrointestinal priming and tube-feeding method. Pediatrics 1999, 103: 434-439.

10. Troche B., Harvey-Wilkes K., Engle W.D., Nielsen H.C., Frantz I.D., Mitchell M.L., and Hermos R.J. Early minimal enteral feedings promote growth in critically ill premature infants. Biol Neonate 1995; 67: $172-181$.

11. Dunn L., Hulman S., Weiner J., and Kliegman R. Beneficial effects of early hypocaloric enteral feeding on neonatal gastrointestinal function: preliminary report of a randomized trial. J Pediatr 1988; 112: $622-629$.

12. Meetze W.H., Valentine C., McGuigan J.E., Conlon M., Sacks N., and Neu J. Gastrointestinal priming prior to full enteral nutrition in very low birth weight infants. J Pediatr Gastroenterol Nutr 1992; 15: 163-170.

13. Berseth C.L. Effect of early feeding on maturation of the preterm infant's small intestine.J Pediatr 1992; 120: 947-953.

14. Slagle T.A. and Gross S.J. Effect of early low-volume enteral substrate on subsequent feeding tolerance in very low birth weight infants. J Pediatr 1988; 113: 526-531.

15. McClure R.J. and Newell S.J. Randomised controlled study of clinical outcome following trophic feeding Arch Dis Child Fetal Neonatal Ed 2000; 82: F29F33.

16. Tyson J.E. and Kennedy K.A. Minimal Enteral Nutrition in Parenterally Fed Neonates. In: Sindair J.C, Bracken M.B., Soll R.F., Horbar J.D., editors. Neonatal Module of The Cochrane Database of Systematic Reviews. Oxford: 1997.

17. Piena M., Albers M.J.I.J., Van Haard P.M.M., Gischler S., and Tibboel D. Introduction of enteral feeding in neonates on extracorporeal membrane axygenation after evaluation of intestinal permeability changes. J Pediatr Surg 1998, 33: 30-34. 
18. Shippee R.L., Johnson A.A., Cioffi W.G., Lasko J., LeVoyer T.E., and Jordan B.S. Simultaneous determination of lactulose and mannitol in urine of burn patients by gas-liquid chromatography. Clin Chem 1992; 38: 343-345.

19. Bjarnason L., MacPherson A., and Hollander D. Intestinal permeability: an overview. Gastroenterology 1995; 108: 1566-1581.

20. Travis S. and Menzies I. Intestinal permeability: functional assessment and significance. Clin Sci 1992; 82: 471-488.

21. Ohri S.K., Somasundaram S., Koak Y., MacPherson A., Keogh B.E., Taylor K.M., Menzies I.S., and Bjarnason $\mathrm{I}$. The effect of intestinal hypoperfusion on intestinal absorption and permeability during cardiopulmonary bypass. Gastroenterology 1994; 106: 318-323.

22. Johnston J.D., Harvey C.J., Menzies I.S., and Treacher D.F. Gastrointestinal permeability and absorptive capacity in sepsis. Crit Care Med 1996; 24: 1144-1149.

23. Gerver W.J.M., Bruin de R., and Blanco C.E. Body proportions of the infant. Biom J 1994; 6: $759-766$.

24. Nusrat A., Turner J.R., and Madara J.L. Molecular physiology and pathophysiology of tight junctions. IV. Regulation of tight junctions by extracellular stimuli: nutrients, cytokines, and immune cells. Am J Physiol 2000; 279: G851-G857.

25. Ferraris R.P. and Diamond J. Regulation of intestinal sugar transport. Physiol Rev 1997; 77: 257-302.

26. Dyer J., Hosie K.B., and Shirazi-Beechy S.P. Nutrient regulation of human intestinal sugar transporter (SGLT1) expression. Gut 1997; 41: 56-59.

27. Thomson A.B.R. and Wild G. Adaptation of intestinal nutrient transport in health and disease (part I). Dig Dis Sci 1997; 42: 453-469.

28. Thomson A.B.R. and Wild G. Adaptation of intestinal nutrient transport in health and disease (part II). Dig Dis Sci 1997; 42: 470-488.

29. Monteiro I.M., Jiang L., and Ferraris R.P. Dietary modulation of intestinal fructose transport and GLUTS mRNA expression in hypothyroid rat pups. J Pediatr Gastroenterol Nutr 1999; 29: 563-570.

30. Catassi C., Bonucci A., Coppa G.V., Carlucci A., and Giorgi P.L. Intestinal permeability changes during the first month: effect of natural versus artificial feeding. J Pediatr Gastroenterol Nutr 1995; 21: 383-386.

31. Shulman R.J., Schanler R.J., Lau C., Heitkemper M., Ou C.-N., and O'Brian Smith E. Early feeding, antenatal glucocorticoids, and human milk decrease intestinal permeability in preterm infants. Pediatr Res 1998; 44: 519-523.

32. Ferraris R.P. and Carey H.V. Intestinal transport during fasting and malnutrition. Annu Rev Nutr 2000; 20: $195-219$. 


\section{CHAPTER 5}

Effect of Repetitive Asphyxia on Leukocyte-Vessel Wall Interactions in the Developing Intestine 


\begin{abstract}
Information on leukocyte-vessel wall interactions (LVWI) during development of the immature intestine is scarce. We therefore designed a new experimental model for studying the microcirculation in the developing intestine of chick foetuses at days $13(n-12), 15(n-17)$, and $17(n-19)$ of incubation $(0.6,0.7$, and 0.8 of the incubation time, respectively) using intravital microscopy. In addition, we investigated whether episodes of asphyxia increase LVWI, and induce tissue damage in the developing intestine. Asphyxia was induced by clamping of the chorioallantoic vein for 6 periods of 5 minutes each, with 5-minute intervals, while in sham groups a sham procedure was performed. Video recordings were made before as well as 10,20 , and 30 minutes after the end of the asphyxia or sham protocol. Baseline number of rolling leukocytes per minute significantly increased $(p<0.001$ ) from 0 at 0.6 incubation to 1.5 and to 4 at 0.7 and 0.8 incubation time, respectively. At 0.6 and 0.7 incubation no adherent leukocytes were observed under baseline conditions, whereas at 0.8 incubation single leukocytes adhered to the venular wall. LVWI variably increased during the course of the experiments. Asphyxia neither enhanced LVWI nor induced histological damage in the intestine. These findings indicate that 1) leukocyte-vessel wall interactions mature during foetal development, and 2) repetitive episodes of asphyxia induce neither an inflammatory response nor histological tissue injury in the developing intestine from 0.6 to 0.8 incubation. We hypothesise that immaturity of leukocyte-vessel wall interactions, as part of the nonspecific host defence to invading bacteria, might play a role in the development of necrotising enterocolitis in preterm neonates.
\end{abstract}




\section{INTRODUCTION}

Hypoxia/reoxygenation injury of the immature intestine after birth has been suggested to play a role in the aetiology of neonatal necrotising enterocolitis (1-3). It has been demonstrated that impairment of intestinal oxygen supply, as a result of interruption of intestinal perfusion, followed by restoration of oxygenation during reperfusion results in necrosis of the intestinal mucosa (4). This tissue injury is partially mediated by activated leukocytes which accumulate into the intestinal interstitium during the reperfusion period $(5,6)$. Intravital microscopic studies have shown that the extravasation of leukocytes is preceded by rolling along and adhesion to the microvascular endothelium $(7,8)$. Previous studies, however, were either conducted in adult or in full-term neonatal animals (9). Since necrotising enterocolitis is predominantly encountered in prematurely born infants, it may be more appropriate in this respect to investigate the effects of intestinal hypoxia/ reoxygenation on induction of leukocyte-vessel wall interactions and tissue damage in a developing intestine.

Research with regard to the effects of hypoxia/reoxygenation on the microvasculature in a developing intestine has been hampered by lack of an adequate animal model. Therefore, the first aim of the present study was to design an experimental model for studying the microcirculation in a developing intestine using intravital microscopy. The chick foetus is especially suited for this purpose, because its intestine is partly located outside the abdominal wall in an omphalocele, thereby enabling noninvase investigation of the intestinal microcirculation at successive stages of foetal development. Furthermore, the chick foetus is commonly used for perinatal studies on cardiovascular (10) and immunological development (11). In the present study we evaluated the development of leukocyte-vessel wall interactions in the intestinal microcirculation of chick foetuses at days 13, 15, and 17 of incubation, which corresponds to $0.6,0.7$, and 0.8 of the incubation time.

A major cause of hypoxia/reoxygenation of a developing intestine may be related to episodes of perinatal asphyxia. It has been demonstrated that asphyxia elicits a cardiovascular response, which is characterised by a redistribution of the cardiac output in favor of the heart and brain, at the expense of peripheral organs, including the intestine (12). A reduction in intestinal blood flow in combination with a diminished arterial oxygen content decreases the oxygen supply to both endothelial and intestinal parenchymal cells. After a period of asphyxia has ended, intestinal perfusion is restored and reoxygenation occurs. In our model we investigated whether repetitive episodes of asphyxia increase leukocyte-vessel wall interactions, and induce histologically detectable damage in the intestinal mucosa at $0.6,0.7$, and 0.8 of chick foetal incubation time. 


\section{Surgical preparation}

Experimental procedures were in accordance with the Dutch law on the use of laboratory animals. Fertile Lohman selected White Leghorn eggs were incubated at $37^{\circ} \mathrm{C}$ and a relative air humidity of $60 \%$, and rotated once every hour (Polyhatch incubator; Brinsea product Ltd., Sandford, England). Incubation time until hatching is 21 days. In this study we used chick foetuses at days 13,15 , and $17(0.6,0.7$, and 0.8 of the incubation time, respectively), corresponding to stages $39-43$ according to Hamilton and Hamburger (13).

Manipulations were performed in a clinical neonatal intensive care incubator (type 7510, Drägerwerk AG, Lübeck, Germany) equipped with a dissecting microscope (Leica MS5, Rijswijk, the Netherlands). During preparation temperature and relative air humidity in the incubator were maintained at $37{ }^{\circ} \mathrm{C}$ and $60 \%$, respectively. The eggs were opened at the blunt side, containing the air cell. After opening the egg by removing part of the eggshell and outer shell membrane, the inner shell membrane was moistened with $0.9 \% \mathrm{NaCl}$ to visualise the vessels of the underlying chorioallantoic membrane (CAM). After penetrating the CAM in an area with sparse vascularisation the omphalocele was localised and centered at the level of the opening by means of two sutures (Ethicon, Prolene 6-0). The omphalocele was opened by careful blunt dissection and the intestine was exposed. One segment of the umbilical loop of the ileum was placed on a $1 \mathrm{~cm}$ piece of cotton tape which was attached to the egg shell. To prevent dehydration a drop of paraffin oil was applied on the exposed intestinal loop after which it was covered with a thin transparant plastic wrapping. Only preparations which required minimal manipulation were included. For further microscopic evaluation in vivo, the egg was transferred into a single-egg chamber, in which temperature $\left(37{ }^{\circ} \mathrm{C}\right)$ and air flow $\left(21 \% \mathrm{O}_{2}\right.$, $4 \mathrm{l} / \mathrm{min}$ ) were controlled, and placed in an intravital microscope setup (Fig. 1).
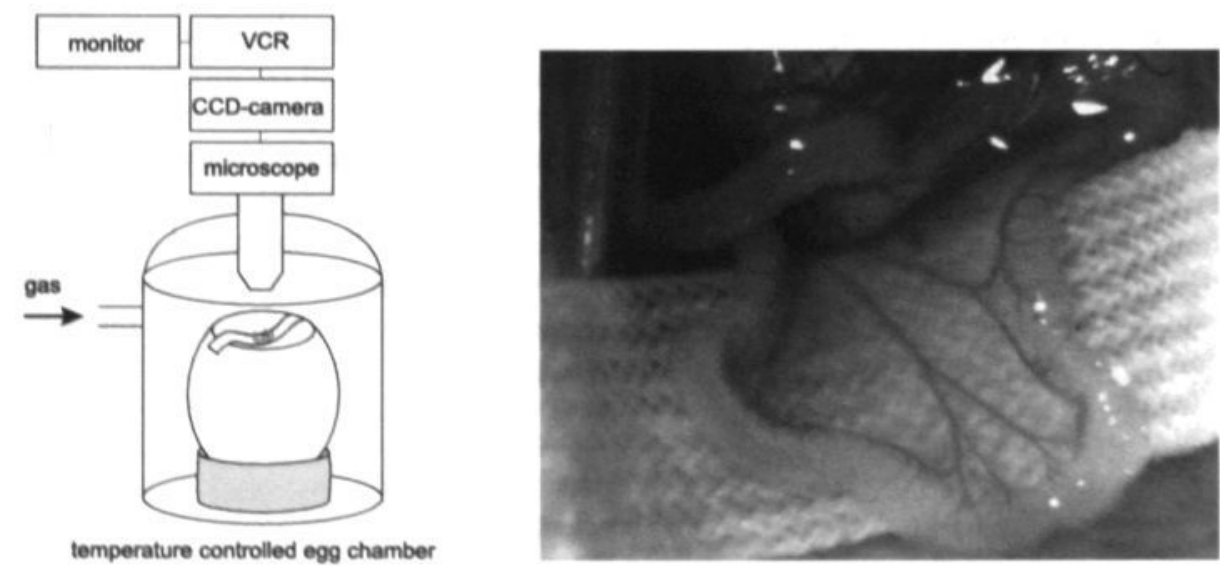

temperature controlled egg chamber

Figure 1. Schematic representation of the intravital microscope setup 


\section{Intravital videomicroscopy}

All in vivo observations were made with an intravital microscope (Leitz Orthoplan 946627). The intestinal microvasculature was visualised with a Leitz $20 x$ long working distance objective (numerical aperture 0.32) via a Leitz Ploemopak illuminator 2.1 (1.25x), equipped with a Leitz POL-cube (14). The intestine was epi-illuminated using a $75 \mathrm{~W}$ Xenon lamp with neutral density filters to reduce the light intensity to the lowest level that yielded good quality of the video image. Images were projected onto a CCD camera with an effective scanned area of $8.3 \times 6.3 \mathrm{~mm}$ (Hamamatsu Photonics, Hamamatsu, Japan), connected to a Super-VHS videorecorder (Panasonic, model NV-FS100HQ), and stored on videotape (Sony, Super VHS VXSE-180Vf). Images were displayed on a monitor screen (Sony, model PVM-122CE). Final resolution was $1 \mu \mathrm{m}$.

\section{Experimental protocol}

Foetuses at predetermined incubation times were randomly divided in 2 groups: asphyxia and sham groups. After an equilibration period of 15 minutes, 2-minute baseline video recordings were made of 2 separate regions of the exposed intestinal loop. In each region one arteriole (diameter $9-34 \mu \mathrm{m}$ ) and a parallel venule $(17-49 \mu \mathrm{m})$ were selected, and these same vessels were observed throughout the experiment. A schematic drawing of the observed region was made to facilitate localisation of the vessels during the course of the experiment. The microvessels were located in the outer layer of the intestinal wall. Selection of vessels was based on optical clarity and, hence, the ability to visualise leukocyte-vessel wall interactions.

Subsequently, the egg was placed in the incubator and repetitive asphyxia was induced in the experimental groups. To this end, the chorioallantoic vein was occluded with a small vessel clamp during 6 periods of 5 minutes each, with intervals of 5 minutes during which the clamp was released. Total duration of this experimental protocol was 60 minutes. In the sham groups, the chorioallantoic vein was manipulated but not occluded, using the same time protocol. Ten, 20, and 30 minutes after the end of the asphyxia or sham protocol, microscopic images of the selected vessels were again recorded on videotape during 2 -minute periods (Fig. 2).

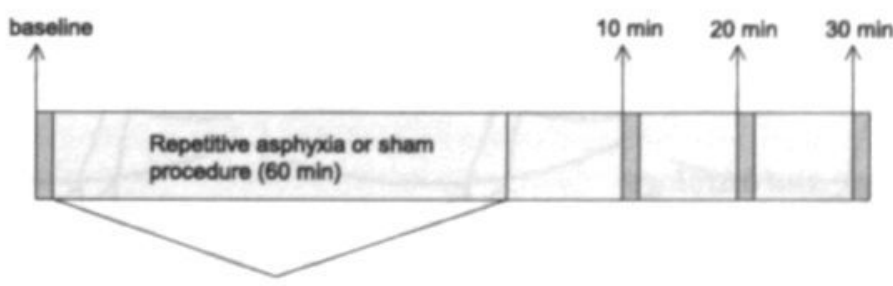

Asphydia groups: $6 \times 5 \mathrm{~min}$ occlusion of CAM vein with intervals of $5 \mathrm{~min}$ Sham groups: sham procedure

D video recording $(202 \mathrm{~min})$

Figure 2. Experimental protocol 
To assess whether clamping of the chorioallantoic vein for 5 minutes indeed induced the biochemical characteristics of asphyxia, we collected blood samples at the end of a 5-minute period of asphyxia in a separate set of experiments at 0.7 foetal incubation time $(n=4)$ and compared the blood gas values with those obtained from a sham group $(n=4)$. Blood samples $(0.2 \mathrm{ml})$ were obtained from the omphalomesenteric artery using a heparinised syringe with a $30 \mathrm{G}$ needle and analysed at $37^{\circ} \mathrm{C}$ (Radiometer ABL510, Copenhagen, Denmark).

In a separate series of foetuses at 0.8 incubation time $(n=4)$, we investigated the effect of topical application of increasing concentrations of $\mathrm{N}$-formyl-methionylleucyl-phenylalanine (FMLP) on leukocyte-vessel wall interactions in intestinal and mesenteric microvessels. FMLP is a synthetic chemoattractant which induces leukocyte adhesion in adult animal models (15). FMLP was dissolved in dimethylsulfoxide (1\% solution) and further diluted in $0.9 \% \mathrm{NaCl}$ to final concentrations of $10^{-7}-10^{-4} \mathrm{M}$. Video recordings of the vessels were made before as well as during 3 minutes after topical application of $20 \mu \mathrm{l}$ aliquots of increasing concentrations $\left(10^{7}-10^{-4} \mathrm{M}\right)$ of FMLP. Solutions were prepared freshly on the day of the experiment.

\section{Experimental parameters}

All experiments were analysed off-line from videotape. The degree of leukocyte rolling was quantified by counting the number of rolling leukocytes passing an imaginary line perpendicular to the venular axis per minute (nRL). Leukocytes were considered to be rolling if they could be seen moving slowly along the venular wall. Rolling leukocytes move at least an order of magnitude slower than free flowing blood cells. Since peristaltic contractions of the intestine occurred with a frequency of approximately 3-4 waves per minute, the microvessels were not always in focus. Rolling leukocytes were only counted between the peristaltic waves; the total counting period lasted 60 seconds per vessel. The average number of adherent leukocytes (nAL) was determined in a $100-\mu \mathrm{m}$ segment of the venule. Leukocytes were considered to be adherent (sticking) when they remained stationary for more than 30 seconds. Arteriolar (Da) and venular (Dv) luminal diameters were measured using an image-shearing device (IPM, model 908, San Diego, CA). Within the selected vessel segment, the diameter was measured three times at the same site; the average was taken as the actual diameter. The data of all experimental parameters obtained from the two observed regions of the intestinal loop in each foetus were averaged.

\section{Survival rate and histology}

In a separate series of experiments the extent of histological damage in the intestine was assessed after repetitive asphyxia (same protocol as for intravital microscopy experiments) at $0.6,0.7$, and 0.8 foetal incubation time ( $n=10$ in each group) and compared with sham foetuses of similar ages ( $n=10$ in each group). Following completion of the asphyxia or sham protocol, eggs were sealed with tape and further incubated for 24 hours. After 24 hours survival rate was determined, and surviving foetuses were sacrificed by means of decapitation. The intestine was removed and placed in buffered $4 \%$ formalin for at least 24 hours. Biopsy speci- 
mens were later dehydrated and embedded in paraffin. Deparaffinised $4 \mu \mathrm{m}$ sections were stained with hematoxylin-eosin stain. The degree of intestinal tissue injury was evaluated on a grading scale from 0 to 8 according to Park et al (4).

\section{Statistical analysis}

Data are presented as median and interquartile range (i.e., spread from 25 th to 75 th percentile). The term $n$ refers to the number of foetuses in which observations were made. In order to evaluate the influence of developmental stage on the experimental parameters, baseline values of sham and asphyxia groups were taken together and compared between the three incubation times. Differences from baseline values were determined by the Wilcoxon signed ranks test, while differences between groups at each time point were determined by the Mann-Whitney $U$ test. Statistical significance was defined as $\mathrm{p}<0.05$.

\section{RESULTS}

Figure 3 shows a schematic picture of the intestinal microcirculation in the chick foetus at $0.6,0.7$, and 0.8 incubation time under baseline conditions. The number of vascular branches in the microcirculation of the outer layer of the intestinal wall increased from 0.6 to 0.8 foetal incubation time. Microscopic observations always were performed in vessels with a diameter of on average $20-30 \mu \mathrm{m}$. Since luminal diameters of both arterioles and venules increased from 0.6 to 0.8 foetal incubation time, we selected vessels of a higher branch order at successive stages of development.

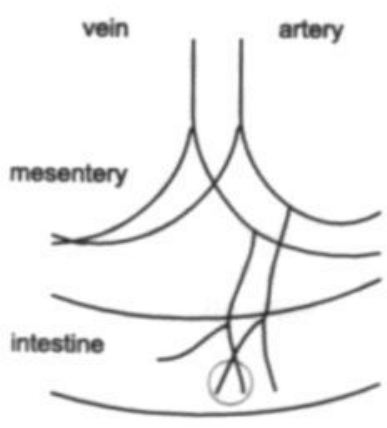

A

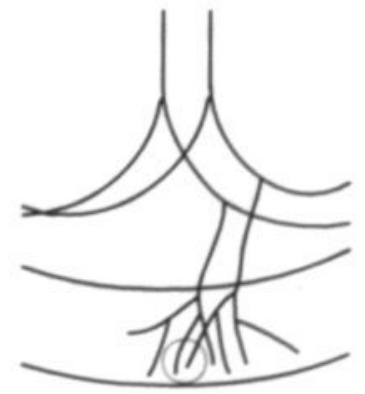

B

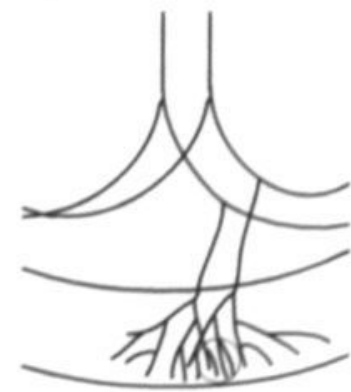

C

Figure 3

Schematic representation of the microcirculation in the outer layer of the chick foetal intestine at $0.6(\mathrm{~A}), 0.7$ (B), and 0.8 (C) foetal incubation time, the site of microscopical observation is indicated by the circle. 


\section{Changes during foetal development}

Leukocyte-vessel wall interactions were only observed in venules, not in arterioles. Baseline values of the numbers of rolling and adherent leukocytes in venules of chick foetuses at $0.6(n=12), 0.7(n=18)$, and $0.8(n=18)$ incubation time are presented in Figure 4. At 0.6 foetal incubation time a single rolling leukocyte was observed in venules of only 2 of the 12 foetuses. In contrast, leukocyte rolling was observed in all venules at 0.7 and 0.8 foetal incubation time. Leukocyte adhesion was never observed under baseline conditions at 0.6 and 0.7 foetal incubation time, whereas in venules of 4 of the 18 foetuses a single adherent leukocyte was observed at 0.8 foetal incubation time.
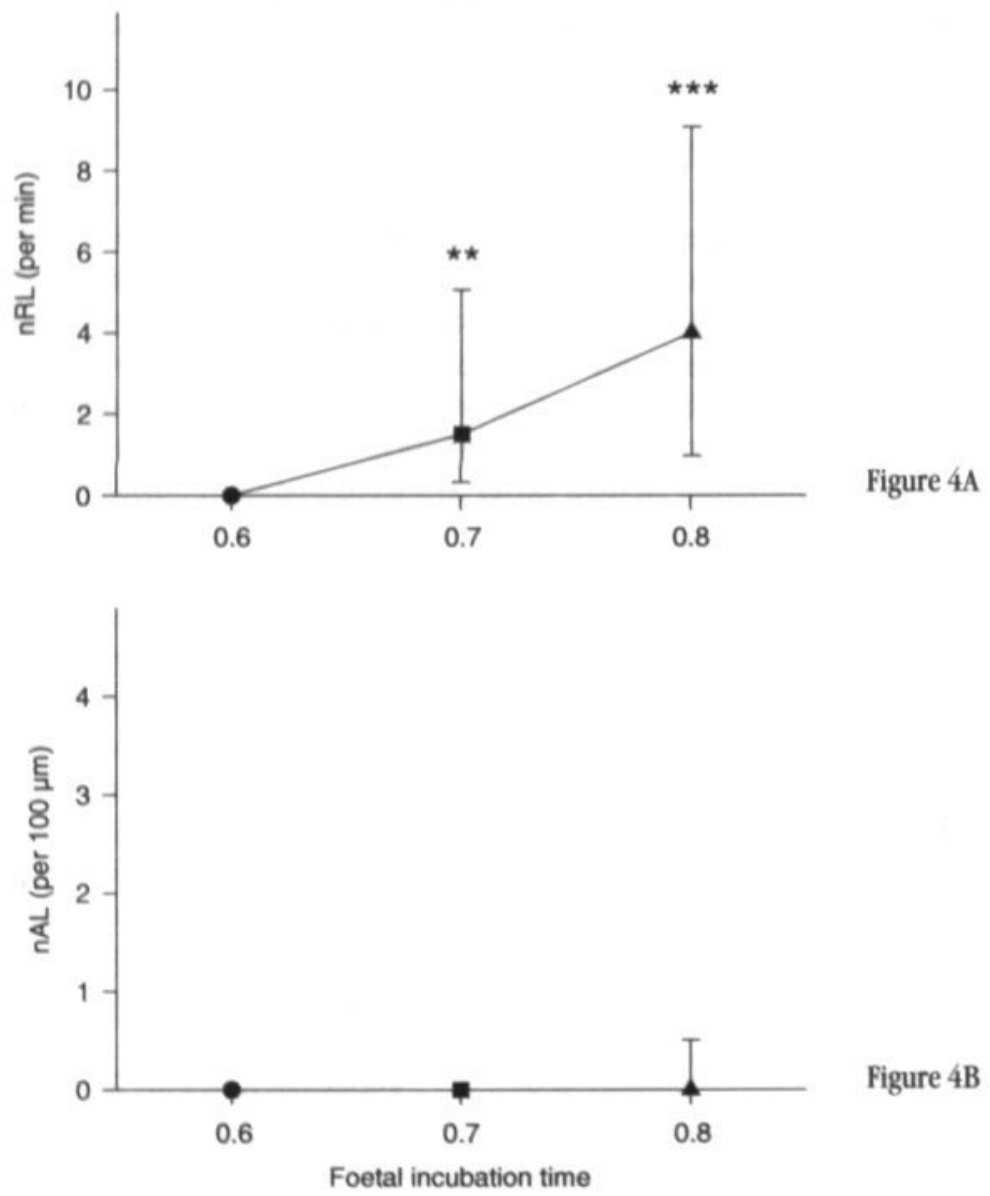

Figure 4

Leukocyte-vessel wall interactions in foetal intestinal venules under baseline conditions. Median number of rolling leukocytes (nRL) per minute (A) and number of adherent leukocytes (nAL) per 100- $\mu$ m venule segment (B) in chick foetuses at $0.6,0.7$, and 0.8 foetal incubation time. Bars denote interquartile ranges. ${ }^{* *} p<0.01,0.7 \mathrm{vs}, 0.6$ foetal incubation time; ${ }^{* * *} p<0.001,0.8 \mathrm{vs}$. 0.6 foetal incubation time 


\section{Effect of asphyxia}

Blood gas analysis in a sham group at 0.7 incubation showed a $\mathrm{PO}_{2}$ of $5.84 \pm 0.50$ $\mathrm{kPa}, \mathrm{PCO}_{2}$ of $4.66 \pm 0.29 \mathrm{kPa}$, and $\mathrm{pH}$ of $7.47 \pm 0.01$. Clamping of the chorioallantoic vein for 5 minutes resulted in a decrease of $\mathrm{Po}_{2}(1.57 \pm 0.44 \mathrm{kPa})$, an increase in $\mathrm{PcO}_{2}$ $(11.25 \pm 0.98 \mathrm{kPa})$, and a decrease in $\mathrm{pH}(7.07 \pm 0.03)$; these blood gas values were significantly different from those of the sham group ( $p<0.05)$.

The effect of repetitive asphyxia on leukocyte rolling in the three age groups is shown in Figure 5. No significant differences in nRL. between sham and asphyxia groups were observed at any incubation time. At 0.6 foetal incubation time the number of rolling leukocytes did not significantly change during the course of the experimental period in either the sham $(n-7)$ or the asphyxia group $(n-5)$. In contrast, at 0.7 foetal incubation time there was a significant time-dependent increase in $\mathrm{nRL}$ in the sham group $(n-8, \mathrm{p}<0.05)$. In the asphyxia group $(n-10)$ at this stage we also observed an increase in $\mathrm{nRL}$, that was only significantly different from baseline at 10 minutes ( $p<0.05$ ) after the end of the asphyxia protocol. At 0.8 foetal incubation time $\mathrm{nRL}$ also increased in both the sham $(n-9)$ and the asphyxia group $(n-9)$, although statistical significance was not reached. At all time-points nRL was significantly higher in the 0.7 and 0.8 incubation groups compared with the 0.6 incubation group $(\mathrm{p}<0.01)$.

Figure 6 demonstrates the effect of repetitive asphyxia on leukocyte adherence. No effect of asphyxia on nAL was observed at any incubation time. Except for the 0.6 incubation groups, the number of adherent leukocytes ( $\mathrm{nAL}$ ) increased during the course of an experiment in the sham as well as the asphyxia groups. This increase reached statistical significance at 10,20, and 30 minutes after the end of the asphyxia or sham protocol at 0.7 incubation, and at 30 minutes in the asphyxia group at 0.8 foetal incubation time. The nAL after 10,20 , and 30 minutes in both the sham and the asphyxia group was significantly higher at 0.7 and 0.8 incubation as compared with 0.6 foetal incubation time ( $p<0.05$ ).

Arteriolar diameters remained constant throughout the experimental period in the sham as well as the asphyxia groups at all three incubation times. Venular diameters also remained fairly constant during the experimental period, although there was a slight decrease in venular diameter at 20 and 30 minutes in the sham group at 0.8 foetal incubation time (from 31 to 24 and $21 \mu \mathrm{m}$, respectively; p<0.05).

\section{Effect of FMLP}

During 3 minutes following topical application of increasing concentrations of FMLP onto the intestine at 0.8 foetal incubation time neither rolling nor adhesion of leukocytes was observed in any of the intestinal or mesenteric venules $(n-4)$

\section{Survival rate and histology}

Survival rates $\mathbf{2 4}$ hours after the asphyxia or sham protocol were approximately $80 \%$ in both groups at all incubation times. None of the surviving foetuses at 0.6 , 0.7 , and 0.8 foetal incubation time from either the sham or the asphyxia group demonstrated any sign of histological damage in the intestine. 


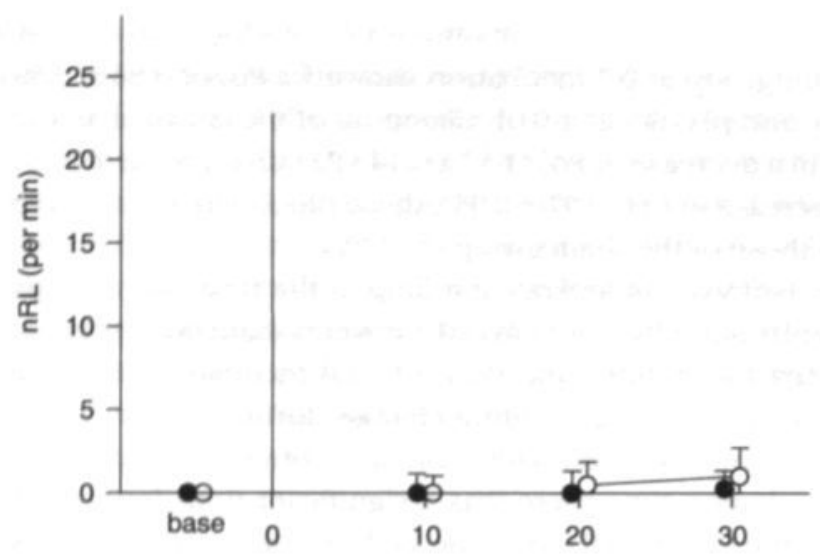

- sham
- asphyxia

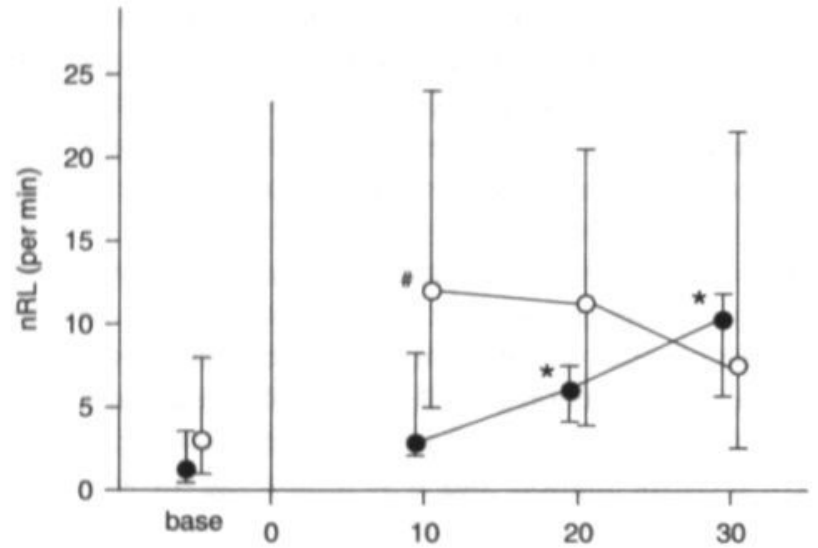

Figure 5A

Figure 5B

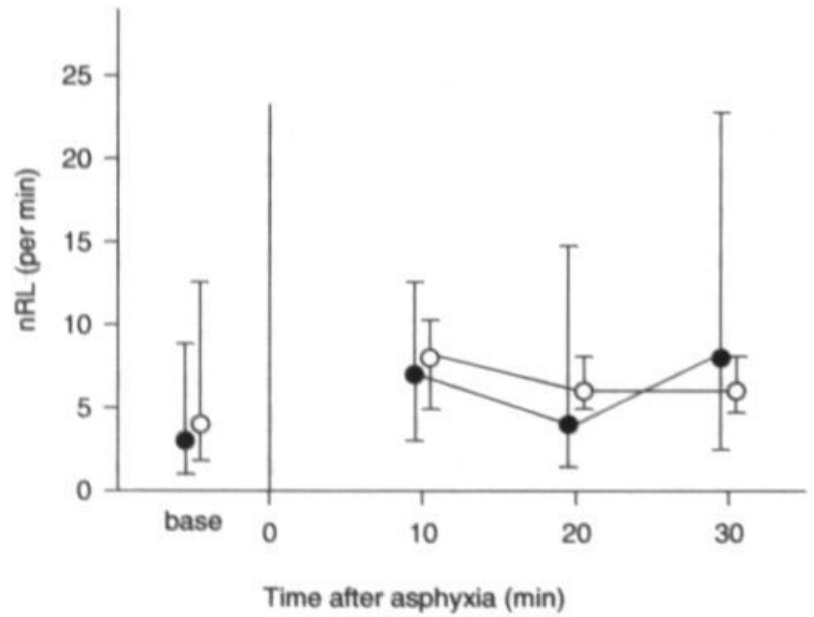

Figure 5C

Figure 5

Effect of repetitive asphyxia on leukocyte rolling in intestinal venules in chick foetuses at $0.6(\mathrm{~A}), 0.7$ (B), and 0.8 (C) foetal incubation time. Median number of rolling leukocytes ( $\mathrm{nRL}$ ) per minute in asphyxia groups and sham groups. Bars denote interquartile ranges.

* $p<0.05$, vs. baseline in sham group; * $p<0.05$, vs. baseline in asphyxia group 


\section{DISCUSSION}

Our study is the first to present a model for in vivo evaluation of leukocyte-vessel wall interactions in the intestinal microcirculation during foetal development. In this model we demonstrated that the level of leukocyte-vessel wall interactions between 0.6 and 0.8 of chick foetal incubation time is low. At 0.6 foetal incubation time rolling or adherent leukocytes were rarely observed. Baseline numbers of rolling leukocytes increased at 0.7 and 0.8 foetal incubation time, which is in contrast to leukocyte adhesion. At these incubation times we also observed an increase in both leukocyte rolling and adhesion during the course of an experiment. Topical application of the chemoattractant FMLP at 0.8 foetal incubation time did not induce leukocyte adhesion. Repetitive episodes of asphyxia did not enhance leukocytevessel wall interactions. Furthermore, no histological damage of the intestinal mucosa was detected 24 hours after the asphyxia protocol.

The baseline number of rolling leukocytes was low, but increased during foetal development. This increment in leukocyte rolling may be attributed to a rising level of circulating leukocytes, or to an increase in the expression and/or function of adhesion molecules on the leukocytes or on the endothelial cells. Total leukocyte count in the chick foetus has been demonstrated to increase with incubation time, from $0.1 \times 10^{9} / \mathrm{A}$ at day 15 ( 0.7 foetal incubation time) to $9 \times 10^{9} / \mathrm{A}$ just before hatching (16). The low circulating leukocyte count at 0.7 foetal incubation time may thus contribute to the rare observation of leukocyte-vessel wall interactions in these young foetuses. In our experimental setup we were not able to determine the type of leukocytes interacting with the venular wall. In adult animals, however, it has been shown that the majority of interacting leukocytes in the mesentery are neutrophils (17). Because the heterophil, which is the avian equivalent of the mammalian neutrophil, is the predominant leukocyte type throughout chick foetal development (16), this seems to be the most likely candidate in our experiments.

Firm adhesion of leukocytes to the venular endothelium under baseline conditions was almost always absent at all three incubational stages. However, at 0.7 and 0.8 foetal incubation time there was an increase to an average of 2 adherent leukocytes per $100 \mu \mathrm{m}$ during the course of an experiment (thus not under baseline conditions), indicating that all attributes necessary for leukocyte adhesion are present at these stages. The discrepancy between the levels of leukocyte rolling and adhesion may be related to the sequence of adhesion molecule expression during foetal development. Only a few studies have addressed the expression and/or function of adhesion molecules involved in leukocyte-vessel wall interactions in the foetus. L-selectin, one of the adhesion molecules that mediates leukocyte rolling, is expressed at adult levels on neutrophils and eosinophils in human foetuses as early as 23 weeks of gestation (18). In contrast, Anderson et al. demonstrated that induction of the surface expression of Mac-1, an adhesion molecule that mediates firm leukocyte adhesion, is significantly lower in neonatal leukocytes compared with adult leukocytes $(19,20)$.

The present study shows that leukocytes of the chick foetus do not adhere to the venular endothelium in response to topical application of FMLP. Leukocyte adherence to the endothelium in response to FMLP is dependent on the presence 


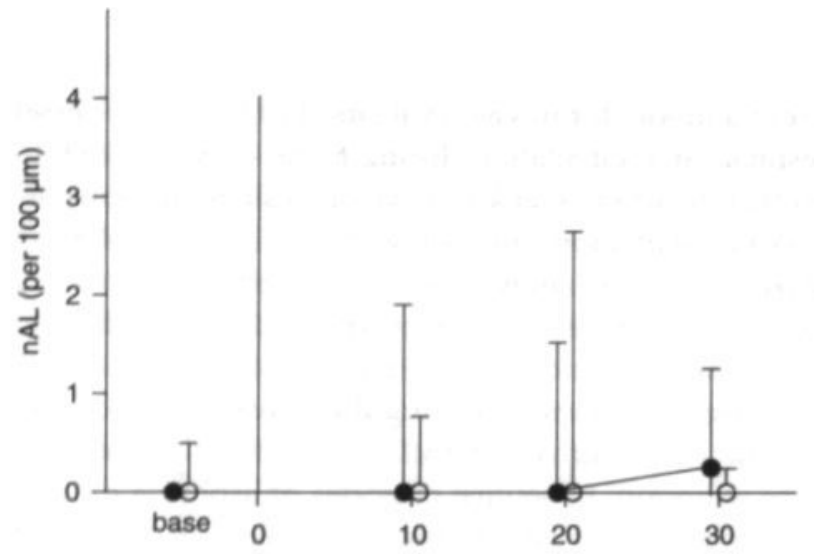

$\begin{array}{ll}- & \text { sham } \\ 0 & \text { asphyxia }\end{array}$

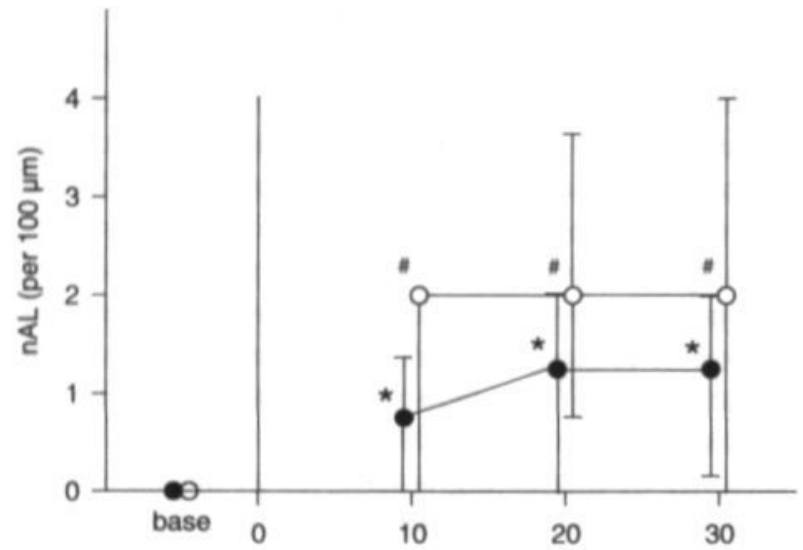

Figure $6 \mathrm{~A}$

Figure 6B

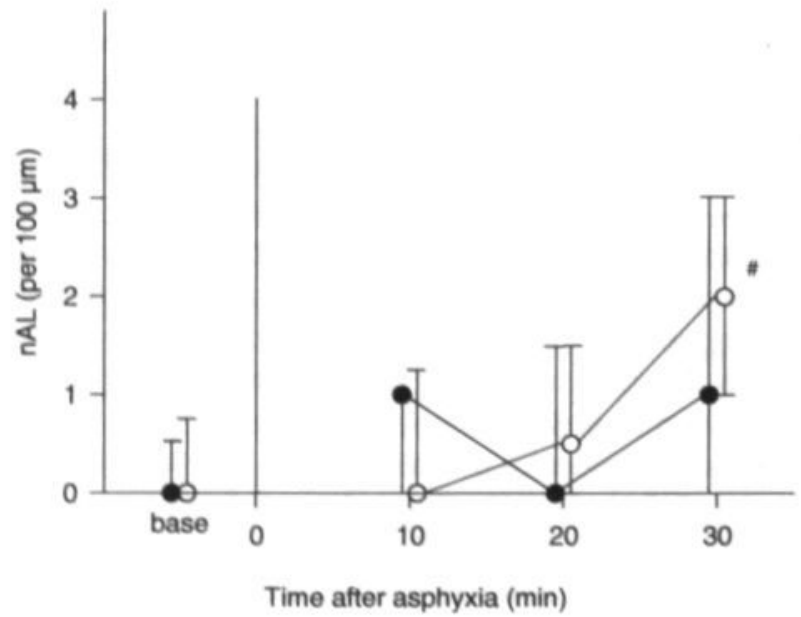

Figure 6C

Figure 6

Effect of repetitive asphyxia on leukocyte adhesion in intestinal venules in chick foetuses at 0.6 (A), 0.7 (B), and $0.8(\mathrm{C})$ foetal incubation time. Median number of adherent leukocytes (nAL) per $100-\mu m$ venule segment in asphyxia groups and sham groups. Bars denote interquartile ranges.

* $\mathrm{p}<0.05, \mathrm{vs}$, baseline in sham group; * $\mathrm{p}<0.05$, vs. baseline in asphyxia group 
of the receptor for this peptide on leukocytes, as well as subsequent expression of leukocyte adhesion molecules. Results of in vitro experiments have shown that FMLP induces chemotaxis of mononuclear leukocytes of adult chickens, which suggests that the receptor for FMLP is present in this avian species, at least in adult animals (21). It is not known whether this receptor is already present in foetal chick leukocytes. Because results of the current study show that leukocyte adhesion can occur, we suggest that failure of FMLP-induced adhesion of leukocytes at 0.8 foetal incubation time is related to a lack of FMLP-receptor expression. Future studies addressing the development of adhesion molecule expression on leukocytes and endothelial cells in the chick foetus may define the underlying mechanisms of these observations.

Repetitive asphyxia enhanced neither leukocyte rolling nor leukocyte adhesion in the foetal intestinal microcirculation at $0.6,0.7$, and 0.8 foetal incubation time compared with sham groups at the respective ages. Although the effect of hypoxia on adhesion molecule expression has been investigated in adult leukocytes and endothelial cells, no studies have addressed this subject in the foetal period. Results of in vitro studies have demonstrated that hypoxia induces the expression of adhesion molecules, such as P-selectin (22), E-selectin (23) and intercellular adhesion molecule-1 (24) on endothelial cells, as well as the expression of the integrin Mac-1 on adult leukocytes (25). Our finding of the absence of enhanced leukocytevessel wall interactions after asphyxia suggests that foetal leukocytes and/or endothelial cells at 0.6 to 0.8 foetal incubation time are not yet able to express adhesion molecules in response to hypoxia. In this respect, the absence of intestinal tissue injury after asphyxia in our experiments is not surprising, because leukocytes generally are believed to be major mediators of tissue injury (6).

An alternative explanation for the absence of enhanced leukocyte-vessel wall interactions after asphyxia may be an increase in circulating levels of various hormones during asphyxia. It has been demonstrated in foetal sheep that acute hypoxia is associated with an increase in circulating glucocorticoids (26). Results of several studies in adult animal models have shown that glucocorticoids suppress leukocyte adhesion and emigration $(27,28)$. It has been shown that glucocorticoids diminish the expression of E-selectin and intercellular adhesion molecule-1 on endothelial cells as well as the expression of integrins on neutrophils (29).

The present study may be of relevance with respect to the aetiology of necrotising enterocolitis in preterm neonates. Our findings that asphyxia did not enhance leukocyte-vessel wall interactions, and failed to induce tissue damage in the developing intestine does not support the hypothesis that injury in an immature intestine, such as in preterm neonates, can be caused by activation and infiltration of leukocytes after episodes of asphyxia. However, in contrast to the sterile developing intestine in our experiments, the immature intestine of preterm neonates is exposed to luminal bacteria and their toxins. Under these circumstances, a limited ability of leukocytes to adhere to the vessel wall and migrate into the intestinal tissue may increase the vulnerability to bacterial invasion. This is even more important considering the fact that it has been demonstrated that the gastrointestinal barrier function is immature in preterm neonates, and that asphyxia may reduce 
clearance of luminal bacteria, thus facilitating bacterial translocation $(30,31)$. Since leukocyte migration into sites of bacterial invasion is fundamental to the host defence response, we hypothesise that immaturity of leukocyte-vessel wall interactions in preterm neonates might play a role in the development of necrotising enterocolitis. 


\section{References}

1. Caplan M.S. and MacKendrick W. Necrotizing enterocolitis: a review of pathogenetic mechanisms and implications for prevention. Pediatr Pathol 1993; 13:357-369.

2. Crissinger K.D. and Granger D.N. Mucosal injury induced by ischaemia and reperfusion in the piglet intestine: influences of age and feeding. Gastmenterology 1989; 97: 920-926.

3. Nowicki P.T. and Nankervis C.A. The role of the circulation in the pathogenesis of necrotizing enterocolitis. Clin Perinatol 1994; 21: 2192233.

4. Park P.O., Haglund U., Bulkley G.B., and Falt K. The sequence of development of intestinal tissue injury after strangulation ischaemia and reperfusion. Surgery 1990; 107: 574-580.

5. Kurtel H., Tso P., and Granger D.N. Granulocyte accumulation in postischemic intestine: role of leukocyte adhesion glycoprotein CD11/CD18. Am J Physiol 1992; 262: G878-6882.

6. Simpson R., Alon R., Kobzik L., Valeri C.R., Shepro D., and Hechtman H.B. Neutrophil and nonneutrophil-mediated injury in intestinal ischaemia-reperfusion. Ann Sung 1993; 218: 444-454.

7. Beuk R.J., Heineman E., Tangelder G.J., Kurvers H.A.J.M., Bonke H.J., and oude Egbrink M.G.A. Effects of different durations of total warm ischaemia of the gut on rat mesenteric microcirculation. J Surg Res 1997; 73:14-23.

8. Granger D.N., Benoit J.N., Suzuki M., and Grisham M.B. Leukocyte adherence to venular endothelium during ischaemia-reperfusion. Am J Physiol 1989, 257: G683-G688.

9. Papparella A.F., DeLuca G., Oyer C.E., Pinar H., and Stonestreet B.S. Ischaemia-reperfusion injury in the intestines of newborn pigs. Pediatr Res 1997; 42: 180-188.

10. Golde van J., Mulder T., and Blanco C.E. Changes in mean chorioallantoic artery blood flow and heart rate produced by hypoxia in the developing chick embryo. Pediatr Res 1997; 42: 293-298.

11. Vainio 0., and Imhof B.A. The immunology and developmental biology of the chicken. Immunology Today 1995; 16: 365-370.

12. Jensen A., Hohmann M., and Kuenzel W. Dynamic changes in organ blood flow and oxygen consumption during acute asphyxia in foetal sheep.J Dev Physiol 1987; 9: 543-559.

13. Hamburger $\mathrm{V}$. and Hamilton H.L. A series of normal stages in the development of the chick embryo. JMorphol 1951; 88: 4998.

14. Slaaf D.W., Tangelder G.J., Reneman R.S., Jager K., and Bollinger A. A versatile incident illuminator for intravital microscopy. Int J Microcinc Clin Exp 1987; 6: 391-397.

15. House S.D. and Lipowsky H.H. In vivo determination of the force of leukocyte endothelium adhesion in the mesenteric microvasculature of the cat. Cin Res 1988; 63: 658668.

16. Romanoff A.L. Handbook: The avian embryo; structural and functional development. New York: The Macmillan Company, 1960.

17. Tangelder G.J., Janssens C.J., Slaaf D.W., oude Egbrink M.G.A., and Reneman R.S. In vivo differentiation of leukocytes rolling in mesenteric postcapillary venules. Am J Physiol 1995; 268: H909H915.

18. Smith J.B. and Tabsh K.M.A. Foetal neutrophils and eosinophils express normal levels of L-selectin. Pediatr Res 1993; 34: 253-257.

19. Anderson D.C., Becker Freeman K.L., Heerdt B., et al. Abnormal stimulated adherence of neonatal granulocytes: impaired induction of surface Mac-1 by chemotactic factors or secretagogues. Blood 1987; 70: $740-750$.

20. Anderson D.C., Rothlein R., Marlin S.D., Krater S.S., and Smith C.W. Impaired transendothelial migration by neonatal neutrophils: abnormalities of Mac-1 (CD11b/CD18)-dependent adherence reactions. Blood 1990; 76: 2613-2621. 
21. Golemboski K.A., Bloom S.E., and Dietert R.R. Dynamics of avian inflammatory response to cross-linked dextran; changes in avian blood leukocyte populations. Inflammation 1990; 14:31-40.

22. Pinsky D.J., Naka Y., Liao H., Oz M.C., Wagner D.D., Mayadas T.N., Johnson R.C., Hynes R.O., Heath M., Lawson C.A., and Stern D.M. Hypoxia-induced exocytosis of endothelial cell Weibel-Palade bodies; a mechanism for rapid neutrophil recruitment after cardiac preservation J Clin Invest 1996; 97: 493-500.

23. Zuend G., Neldon D.P., Neufeld E.J., Dzus A.L., Bischoff J., Mayer J.E., and Colgan S.P. Hypoxia enhances stimulus-dependent induction of E-selectin on aortic endothelial cells. Proc Natl Acad Sci USA 1996; 93: 7075-7080.

24. Shreeniwas R., Koga S., Karakurum M., Pinsky D., Kaiser E., Brett J., Wolitzky B.A., Norton C., Plocinski J., Benjamin W., Burns D.K., Goldstein A., and Stern D. Hypoxia-mediated induction of endothelial cell interleukin-1a J Clin Invest 1992; 90: 2333-2339.

25. Montoya M.C., Luscinskas F.W., del Pozo M.A., Aragones J., and de Landazuri M.0. Reduced intracellular oxidative metabolism promotes firm adhesion of human polymorphonuclear leukocytes to vascular endothelium under flow conditions. Eur J Immunol 1997; 27: 1942-1951.

26. Jackson B.T., Morrison S.H., Cohn H.E., and Piasecki G.J. Adrenal secretion of glucocorticoids during hypoxemia in foetal sheep. Endocrinology 1989; 125: 2751-2757.

27. Mizobe K., Kishihara K., El-Naggar R.E.Z., Madkour G.A., Kubo C., and Nomoto K. Restraint stressinduced elevation of endogenous glucocorticoid suppresses migration of granulocytes and macrophages to an inflammatory locus. J Neuroimmunol 1997; 73: 81-89.

28. Tailor A., Flower R.J., and Perretti M. Dexamethasone inhibits leukocyte emigration in rat mesenteric post-capillary venules: an intravital microscopy study. J Leukoc Biol 1997; 62: 301-308.

29. Filep J.G., Delalandre A., Payette Y., and Foldes-Filep E. Glucocorticoid receptor regulates expression of L-selectin and CD11/CD18 on human neutrophils. Circulation 1997; 96: 295-301.

30. Steinwender G., Schimpl G., Sixl B., Kerbler S., Ratschek M., Kilzer S., Hollwarth M.E., and Wenzl H.H. Effect of early nutritional deprivation and diet on translocation of bacteria from the gastrointestinal tract in the newborn rat. Pediatr Res 1996; 39: 415-420.

31. Van Camp J.M., Tomaselli V., and Drongowski R. Bacterial translocation in the newborn rabbit: effect of age on frequency of translocation. Pediatr Surg Int 1995; 10: 134137. 


\title{
CHAPTER 6
}

\author{
Development of Vasomotor Responses \\ in Foetal Mesenteric Arteries
}

Alles stroomt, en niets staat stil. (Herakleitos van Ephese, Ge eeuw v. Chr.) 


\begin{abstract}
Changes in mesenteric arterial diameters were studied using intravital microscopy in chick foetuses at days 13 and 17 of incubation, corresponding to 0.6 and 0.8 foetal incubation time, both during 5 minutes of hypoxia followed by 5 minutes of reoxygenation, and after topical administration of increasing concentrations $\left(10^{6}-10^{-2} \mathrm{M}\right)$ of norepinephrine and acetylcholine. Baseline diameters of second order mesenteric arteries increased from $56 \mu \mathrm{m}$ at 0.6 incubation to $75 \mu \mathrm{m}$ at 0.8 incubation. Acute hypoxia induced a reduction in arterial diameter to $87 \pm 4.4 \%$ of baseline at 0.6 incubation and to $44 \pm 6.7 \%$ at 0.8 incubation $(p<0.01)$. During reoxygenation, mesenteric arteries dilated to $118 \pm 6.5 \%$ and $121 \pm 7.5 \%$ of baseline at 0.6 and 0.8 foetal incubation time, respectively. Phentolamine did not affect the vasoconstriction during hypoxia at 0.6 incubation, whereas the $\alpha$-adrenergic antagonist significantly attenuated the vasoconstrictor response at 0.8 incubation (to $93 \pm 2.7 \%$ of baseline, $p<0.01$ ). Topical norepinephrine induced maximal vasoconstriction to $71 \pm 3.0 \%$ of baseline at 0.6 incubation and to $35 \pm 3.8 \%$ at 0.8 incubation $(p<0.01)$. Maximal vasodilation to topical acetylcholine was $113 \pm 4.4 \%$ and $122 \pm 4.8 \%$ of baseline at 0.6 and 0.8 incubation, respectively. These in vivo findings show that foetal mesenteric arteries constrict in response to acute hypoxia, and that the increase in magnitude of this vasoconstrictor response from 0.6 to 0.8 of foetal development results from an increase in adrenergic constrictor capacity.
\end{abstract}




\section{INTRODUCTION}

Neonatal necrotising enterocolitis is a clinical condition which is characterised by necrosis of the neonatal intestine. An imbalance between oxygen consumption of and arterial oxygen supply to the intestine has been implicated in the pathophysiology of this disease $(1,2)$. Previous studies with regard to circulatory physiology of the neonatal intestine were conducted in piglets during the first month after birth. However, necrotising enterocolitis is predominantly observed in preterm neonates, with an increased incidence with decreasing gestational age at birth. Since arterial oxygen supply depends in part on the capability to regulate arterial diameter, insight into the regulation of mesenteric arterial tone in the foetal developing intestine may contribute to our understanding of the pathophysiology of this disease.

Information regarding onset and nature of the regulation of arterial tone during foetal development may be derived from experimental studies conducted in foetal sheep and chick foetuses, which addressed the redistribution of the cardiac output during an acute reduction of the arterial oxygen content at consecutive stages of foetal gestation $(3,4)$. It was demonstrated that acute hypoxia induced a decrease in intestinal blood flow at 0.9 of foetal gestation in both species $(4,5)$. Since both administration of the $\alpha$-adrenergic antagonist phenoxybenzamine (6) and chemical sympathectomy using 6-hydroxydopamine (5) blunted this reduction in intestinal blood flow, it was postulated that circulating catecholamines or sympathetic nerves may be involved in the control of intestinal arterial tone at this stage of foetal gestation. However, interpretation of these observations with respect to the regulation of intestinal arterial tone is hampered by the fact that in these studies intestinal blood flow was measured, by means of a microsphere technique or flow probe, instead of actual arterial diameter. According to Poiseuille's law, blood flow is determined by vascular resistance and blood pressure. Hence, a reduction in intestinal blood flow during hypoxia may be caused by an increase in intestinal arterial resistance, and/or by a decrease in arterial pressure. The latter may be due to a reduction of cardiac output or a resistance decrease in cerebral or myocardial vascular beds (shunting). Additionally, changes in blood pressure may also directly influence arterial diameter through alteration of the myogenic tone of the vessel. In order to discern between these mechanisms responsible for a reduction in intestinal blood flow, it is necessary to measure both intestinal arterial diameter and blood pressure during acute hypoxia.

We designed an experimental setup using intravital microscopy to investigate changes in mesenteric arterial diameters in vivo in response to physiological and pharmacological stimuli in the developing chick foetus. The intestine of the chick foetus is partly located outside the abdomen in the naturally occurring omphalocele, hence the mesenteric arteries can be studied without extensive invasive surgery and general anaesthesia, thereby avoiding any influence of anaesthetics on arterial tone (7). The study was conducted at days 13 and 17 of incubation, which corresponds to 0.6 and 0.8 foetal incubation time. It has been suggested that regulation of arterial tone may be initiated in this period of foetal development (8). First, we determined the changes in mesenteric arterial diameter during an acute 
reduction in foetal oxygen supply (hypoxia), and the subsequent response after restoring oxygen delivery (reoxygenation). In order to elucidate the possible role of $\alpha$-adrenoceptors in this response, experiments were also performed during $\alpha$-adrenergic blockade. Since acute hypoxia in the chick foetus is associated with a rise in plasma levels of norepinephrine (9), we subsequently investigated the effect of exogenously applied norepinephrine on mesenteric arterial diameter. The vasodilator capacity of the mesenteric arteries at 0.6 and 0.8 foetal incubation time was evaluated by measuring changes in vascular diameter in response to acetylcholine.

\section{MATERIALS AND METHODS}

\section{Surgical preparation}

Experimental procedures were in accordance with the Dutch law on the use of laboratory animals. Fertile Lohman selected White Leghorn eggs were incubated at $37^{\circ} \mathrm{C}$, a relative air humidity of $60 \%$, and rotated once every hour (Polyhatch incubator; Brinsea product Ltd., Sandford, UK). Incubation time until hatching for these eggs is 21 days. In this study we used chick foetuses at days 13 and 17 of incubation ( 0.6 and 0.8 of foetal incubation time, respectively), corresponding to stages 39 and 42 according to Hamilton and Hamburger (10).

Surgical preparations were performed in a clinical intensive care incubator (type 7510, Drägerwerk AG, Lübeck, Germany) equipped with a dissecting microscope (Leica MS5, Rijswijk, the Netherlands), while temperature and relative air humidity were maintained at $37^{\circ} \mathrm{C}$ and $60 \%$, respectively. The eggs were opened at the blunt end containing the air cell. After removing part of the egg shell and outer shell membrane, the inner shell membrane was moistened with $0.9 \% \mathrm{NaCl}$ to visualise the vessels of the underlying chorioallantoic membrane (CAM). After penetrating the CAM in an area with sparse vascularisation, avoiding bleeding, the
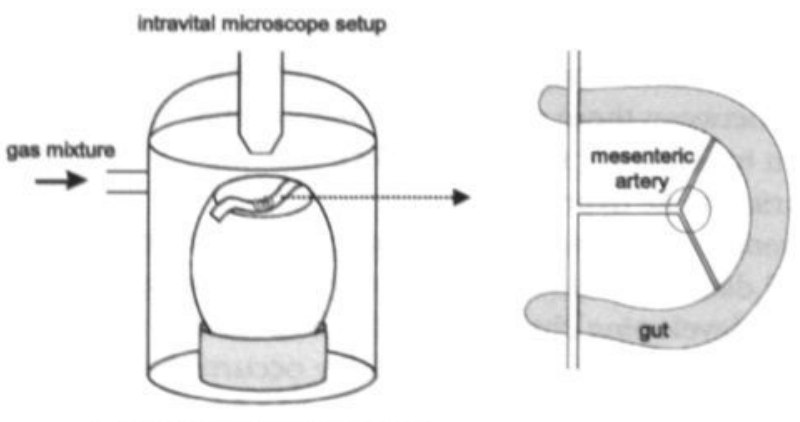

temperature controlled egg chamber

\section{Figure 1}

Schematic representation of the intravital microscopy setup (left); top view of foetal mesenteric preparation with site of observation in circle (right). 
omphalocele was localised and centered at the level of the CAM by means of two sutures through the connective tissue (Ethicon, Prolene 6-0). The omphalocele was opened by careful blunt dissection and the intestine was exposed. One segment of the umbilical loop of the ileum with its mesentery was placed on a $1 \mathrm{~cm}$ piece of cotton tape which was attached to the egg shell. Only preparations which were completed within 15 minutes after opening the egg and which required minimal manipulation during positioning were included in the experimental protocol. For further microscopic evaluation in vivo, the egg was transferred to a singleegg chamber where temperature $\left(37^{\circ} \mathrm{C}\right)$ and air flow $(4 \mathrm{~V} / \mathrm{min})$ were controlled, and subsequently placed in an intravital microscope setup (Fig. 1).

\section{Intravital videomicroscopy}

All in vivo observations were made with an intravital microscope (Leitz Orthoplan 946627). Visualisation of the vasculature was performed with a Leitz 20x long working distance objective (numerical aperture 0.32) via a Leitz Ploemopak illuminator 2.1 (1.25x), equipped with a Leitz POL-cube (11). The intestine was epiilluminated using a $75 \mathrm{~W}$ Xenon lamp. Images were projected onto a CCD camera (Hamamatsu Photonics, Hamamatsu, Japan), connected to a Super-VHS videorecorder (Panasonic, model NV-FS100HQ), and stored on videotape (Sony, Super VHS VXSE-180Vf). Images were displayed on a monitor screen (Sony, model PVM$122 \mathrm{CE})$. Final resolution was $1 \mu \mathrm{m}$. Arterial diameters were analysed off-line using an image-shearing device (IPM, model 908, San Diego, CA). All experiments were performed on anatomically similar locations of second order branches of the omphalomesenteric artery.

\section{Drugs and solutions}

Norepinephrine (l-artenolol-bitrate, NE), acetylcholine (ACh), sodium nitroprusside, adenosine, papaverine, and phentolamine (PHENT) were obtained from Sigma Chemical Co. All drugs were dissolved in HEPES buffered Krebs with the following composition (in mM): $\mathrm{NaCl} 143.3, \mathrm{KCl} 4.7, \mathrm{MgSO}_{4} 1.2, \mathrm{KH}_{2} \mathrm{PO}_{4} 1.2, \mathrm{CaCl}_{2}$ 2.5 , glucose 5.6, HEPES 15. The pH of the buffer was adjusted to 7.4. Final concentrations of the solutions ranged from $10^{-6}$ to $10^{-2} \mathrm{M}$. All drugs were freshly prepared on the day of the experiment; temperature of the solutions at the time of administration was $37^{\circ} \mathrm{C}$.

\section{Experimental protocol}

\section{Effect of acute hypoxia}

Evaluation of the effect of acute hypoxia on second order mesenteric arterial diameters was performed at $0.6(n-8)$ and $0.8(n-7)$ foetal incubation time. After an equilibration period of 15 minutes following surgical preparation, a 2-minute baseline recording was made. Subsequently, hypoxia was induced by replacing the ambient air $\left(21 \% \mathrm{O}_{2}\right)$ in the egg chamber by $100 \% \mathrm{~N}_{2}(4 \mathrm{l} / \mathrm{min})$ for 5 minutes, according to a method described previously (4). After completion of the hypoxic period, reoxygenation was achieved by replacing the $\mathrm{N}_{2}$ with ambient air. During this period one mesenteric artery was continuously kept in focus and recorded. 
To verify whether this protocol actually reduced blood oxygen content, i.e., resulted in hypoxaemia, blood samples were collected at the end of the 5-minute period of hypoxia in a separate series of chick foetuses at $0.6(n=6)$ and $0.8(n=7)$ foetal incubation time and blood gas values were compared with control groups under normoxic conditions. Samples $(0.2 \mathrm{ml})$ were obtained by withdrawing blood from the chorioallantoic vein using a $1 \mathrm{ml}$ heparinised syringe attached to a $21 \mathrm{G}$ needle and analysed at $37^{\circ} \mathrm{C}$ using a blood gas analyser (Radiometer ABL510, Copenhagen, Denmark). The chorioallantoic vein, being the avian equivalent of the mammalian umbilical vein, transports blood from the chorioallantoic membrane (where gas exchange takes place) to the foetus. Therefore, changes in chorioallantoic vein blood gas values reflect changes in foetal arterial blood gas values.

\section{Blood pressure measurements}

In an additional series of experiments at $0.6(n-5)$ and $0.8(n-6)$ foetal incubation time, mean arterial pressure and heart rate were determined under normoxic conditions as well as during a 5-minute period of hypoxia. The technique for measuring blood pressure in the chick foetus was adapted from a method previously described by Tazawa et al., and has been demonstrated to provide reliable blood pressure measurements while maintaining foetal gas exchange (12). Briefly, after opening of the egg in the clinical incubator, one of the two branches of the chorioallantoic artery was cannulated with a $10-\mathrm{cm}$-long nylon catheter (internal diameter $0.5 \mathrm{~mm}$ ) which was filled with $0.9 \% \mathrm{NaCl}$. The catheter was inserted into the artery with its tip pointing upstream. The free end of the catheter was connected to a pressure transducer (Baxter Uniflow, Baxter B.V., Uden, the Netherlands) which was placed at the same height as the egg. Pressure signals were recorded on a computer using a data acquisition system and the heart rate was calculated.

\section{Effect of topically applied phentolamine}

The effect of $\alpha$-adrenergic blockade on vasomotor responses to acute hypoxia was assessed in chick foetuses at $0.6(n=8)$ and $0.8(n=8)$ foetal incubation time. After equilibration and baseline recording a $20 \mu \mathrm{l}$ aliquot of a $10^{-3} \mathrm{M}$ phentolamine solution (PHENT), corresponding to a single dose of $6.35 \mu \mathrm{g}$, was applied to the mesenteric arteries. During the 5 minutes after application of phentolamine, a recording was made to assess the effect of $\alpha$-adrenergic blockade on baseline arterial diameter. Subsequently, hypoxia was induced for 5 minutes followed by 5 minutes of reoxygenation. During this period the same mesenteric artery was continuously kept in focus and recorded.

\section{Effect of topically applied norepinephrine}

In a separate series of chick foetuses at $0.6(n=8)$ and $0.8(n-10)$ foetal incubation time, we assessed constrictor responses of the mesenteric arteries to norepinephrine (NE). After equilibration and baseline recording a cumulative log molar concentration-response curve was constructed by applying $20 \mu \mathrm{l}$ aliquots of increasing concentrations $\left(10^{-6}-10^{-2} \mathrm{M}\right)$ of $\mathrm{NE}$ to the mesenteric arteries, corresponding to single doses of $6.38 \mathrm{ng}-63.8 \mu \mathrm{g}$. After application of each dose a 2-minute recording was made. 
To verify the efficacy of the $\alpha$-adrenergic blockade we also constructed a concentration-response curve for NE 5 minutes after topical administration of a $20 \mu \mathrm{l}$ aliquot of a $10^{-3} \mathrm{M}$ phentolamine solution to the mesenteric arteries in a separate series of chick foetuses at $0.6(n-6)$ and $0.8(n-6)$ foetal incubation time.

\section{Effect of topically applied acetylcholine}

To assess the vasodilator capacity of second order mesenteric arteries both under baseline conditions and after induction of arterial tone using $10^{-2} \mathrm{M}$ topically applied NE, we measured changes in mesenteric arterial diameters in response to topically applied acetylcholine (ACh). Dilator responses under baseline conditions were assessed in chick foetuses at $0.6(n-6)$ and $0.8(n-7)$ foetal incubation time. After equilibration and baseline recording a cumulative log molar concentrationresponse curve was constructed by applying $20 \mu$ aliquots of increasing concentrations $\left(10^{6}-10^{-2} \mathrm{M}\right)$ of $\mathrm{ACh}$ to the mesenteric arteries, corresponding to single doses of $3.63 \mathrm{ng}-36.3 \mu \mathrm{g}$. After each dose a 2-minute recording was made. Dilator responses in NE-constricted arteries were assessed in a similar way by topical application of $20 \mu \mathrm{l}$ aliquots of $\mathrm{ACh} 10^{6}-10^{-2} \mathrm{M}$ after administration of $10^{-2} \mathrm{M} \mathrm{NE}$ to the mesenteric arteries.

In a separate series of chick foetuses at 0.8 foetal incubation time $(n-5)$, we investigated whether topical application of a $20 \mu \mathrm{l}$ aliquot of $10^{-2} \mathrm{M} \mathrm{ACh}$ induced maximal vasodilation. To this end, the vasodilator effect of $10^{-2} \mathrm{M} \mathrm{ACh}$ was compared to a cocktail of $10^{-2} \mathrm{M}$ each of sodium nitroprusside, adenosine, and papaverine. No additional increment in arterial diameter was observed after application of this cocktail on top of ACh (data not shown), indicating that a $20 \mu \mathrm{l}$ aliquot of $10^{-2} \mathrm{M}$ ACh induced maximal vasodilation.

The effect of repetitive application of the buffer solution was assessed in a separate series of chick foetuses at 0.8 foetal incubation time $(n-5)$. To this end, $20 \mu \mathrm{l}$ aliquots of HEPES buffered Krebs were applied to the mesenteric arteries 10 times, with 2-minute intervals. No significant changes in arterial diameters were observed during 10 successive applications of $20 \mu \mathrm{l}$ of HEPES buffered Krebs within a 30-minute period (data not shown).

\section{Quantification of arterial responses}

Luminal diameter of a second order mesenteric artery was measured at about 50 $\mu \mathrm{m}$ from the bifurcation of the first order mesenteric artery, a site with clear distinction of the inner margins of the vessel wall (13). In the course of an experiment, all measurements were performed at this site of the second order mesenteric artery. Changes in diameter are presented as percentage of baseline diameter, with baseline being $100 \%$.

\section{Data analysis}

Data are expressed as mean $\pm \mathrm{SEM}$. The term $n$ refers to the number of foetuses; one artery per foetus was selected. Statistical comparisons between groups were made using the Mann-Whitney U test. Statistical comparisons within groups were made using the Wilcoxon signed ranks test. Statistical significance was defined as a $p<0.05$. 

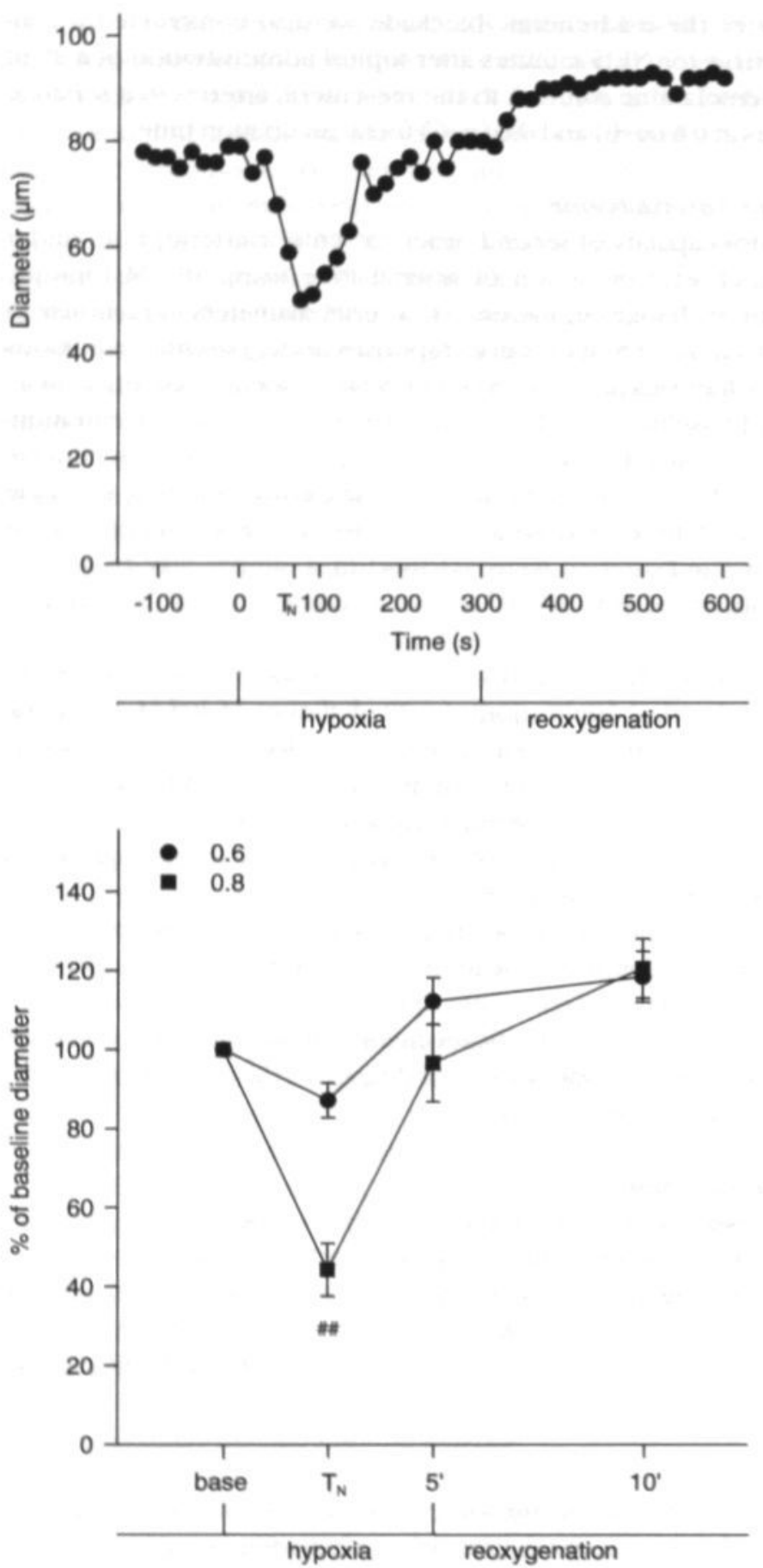

Figure 2

Diameter tracing of the effect of hypoxia and reoxygenation on a second order mesenteric artery at 0.8 foetal incubation time. Arterial diameter was measured every 15 seconds, under baseline conditions $(-120-0 \mathrm{~s})$, during hypoxia (0-300 s), and during reoxygenation $(300-600$ s); $\mathrm{T}_{\mathrm{N}}$ indicates timepoint of maximal vasoconstriction.

Figure 3

Effect of 5 minutes of hypoxia followed by $5 \mathrm{~min}$ utes of reoxygenation on mesenteric arterial diameter at 0.6 and 0.8 foetal incubation time. Acute hypoxia induced a significant reduction in arterial diameter at both 0.6 and 0.8 foetal incubation time. The magnitude of the vasoconstriction was significantly larger in the older foetuses. During reoxygenation a similar level of vasodilation above baseline was observed at both stages of foetal incubation. *t $\mathrm{p}<0.01,0.6$ vs. 0.8 foetal incubation time 


\section{RESULTS}

\section{Effect of acute hypoxia}

Figure 2 illustrates a diameter tracing of the effect of acute hypoxia on a second order mesenteric artery at 0.8 foetal incubation time. The artery was recorded continuously and arterial diameter was measured every 15 seconds. The response to hypoxia was characterised by an initial transient constriction with a peak after about 100 seconds, followed by a relaxation to baseline level that continued until the end of the 5-minute hypoxic period. Reoxygenation resulted in a vasodilation above baseline, which was maintained until the end of the 5-minute period of reoxygenation.

In subsequent experiments, mesenteric arterial diameters were measured at four time points: before hypoxia (base), at maximal vasoconstriction during hypoxia $\left(\mathrm{T}_{\mathrm{N}}\right)$, at the end of the hypoxic period $\left(5^{\prime}\right)$, and at the end of reoxygenation $\left(10^{\prime}\right)$. Baseline diameters increased from $56 \pm 2.9 \mu \mathrm{m}$ at 0.6 incubation to $75 \pm 2.4$ $\mu \mathrm{m}$ at 0.8 foetal incubation time. During hypoxia arterial diameters significantly decreased to $87 \pm 4.4 \%$ of baseline (from 56 to $49 \mu \mathrm{m}$, p<0.05) at 0.6 foetal incubation time and to $44 \pm 6.7 \%$ (from 64 to $28 \mu \mathrm{m}, \mathrm{p}<0.05$ ) at 0.8 foetal incubation time (Fig. 3). The magnitude of this vasoconstrictor response was fourfold larger at 0.8 as compared with 0.6 foetal incubation time $(p<0.01)$. Maximal vasoconstriction during the 5-minute period of hypoxia was observed at 106 (51-207) seconds and 105 (79-137) seconds from the start of hypoxia at 0.6 and 0.8 foetal incubation time, respectively, and lasted for approximately 30 seconds. During reoxygenation a significant vasodilation above baseline was observed in both groups (to $118 \pm 6.5 \%$ and $121 \pm 7.5 \%$ of baseline, $\mathrm{p}<0.05$, at 0.6 and 0.8 foetal incubation time, respectively).

Blood gas analysis demonstrated that 5 minutes of hypoxia induced a significant decrease of arterial $\mathrm{Po}_{2}$ from 11.7 to $3.0 \mathrm{kPa}$ at 0.6 foetal incubation time, and a reduction of arterial $\mathrm{Po}_{2}$ from 7.2 to $1.6 \mathrm{kPa}$ at 0.8 foetal incubation time. The absolute level of the arterial $\mathrm{Po}_{2}$ at the end of the 5-minute period of hypoxia was higher at 0.6 as compared with 0.8 foetal incubation time. However, when expressed as a percentage of control (normoxic) oxygen tension, arterial $\mathrm{PO}_{2}$ was reduced to a similar extent at 0.6 and 0.8 foetal incubation time ( $74 \%$ and $78 \%$, respectively, Table 1 ).

Table 1. Blood gas values at 0.6 and 0.8 foetal incubation time at the end of the 5 - minute period of hypoxia $\left(100 \% \mathrm{~N}_{2}\right)$ as compared with control conditions $\left(21 \% \mathrm{O}_{2}\right)$

\begin{tabular}{lllll}
\hline & \multicolumn{2}{l}{0.6 foetal incubation time } & \multicolumn{2}{l}{$\mathbf{0 . 8 \text { foetal incubation time }}$} \\
& control $(n-5)$ & hypoxia $(n-5)$ & control $(n-7)$ & hypoxia $(n-7)$ \\
\hline $\mathrm{PO}_{2}(\mathrm{kPa})$ & $11.65 \pm 0.63$ & $2.98 \pm 0.26^{*}$ & $7.18 \pm 0.59$ & $1.57 \pm 0.18^{* *}$ \\
$\mathrm{PcO}_{2}(\mathrm{kPa})$ & $1.98 \pm 0.07$ & $1.43 \pm 0.04^{*}$ & $4.57 \pm 0.41$ & $4.45 \pm 0.25$ \\
$\mathrm{pH}$ & $7.57 \pm 0.02$ & $7.63 \pm 0.03$ & $7.56 \pm 0.03$ & $7.53 \pm 0.03$ \\
\hline
\end{tabular}

$\because \cdots p<0.05, p<0.01$, at the end of 5 minutes of hypoxia vs. control 


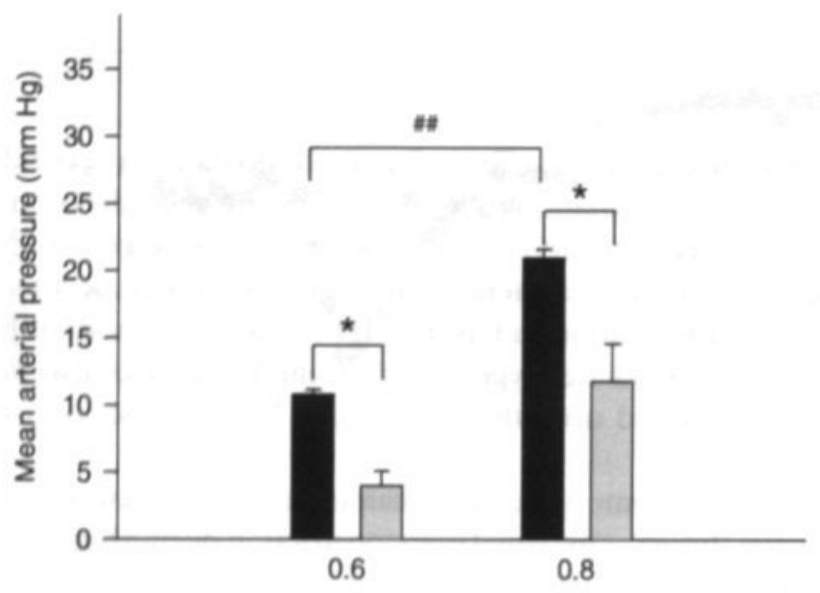

Figure $4 \mathrm{~A}$

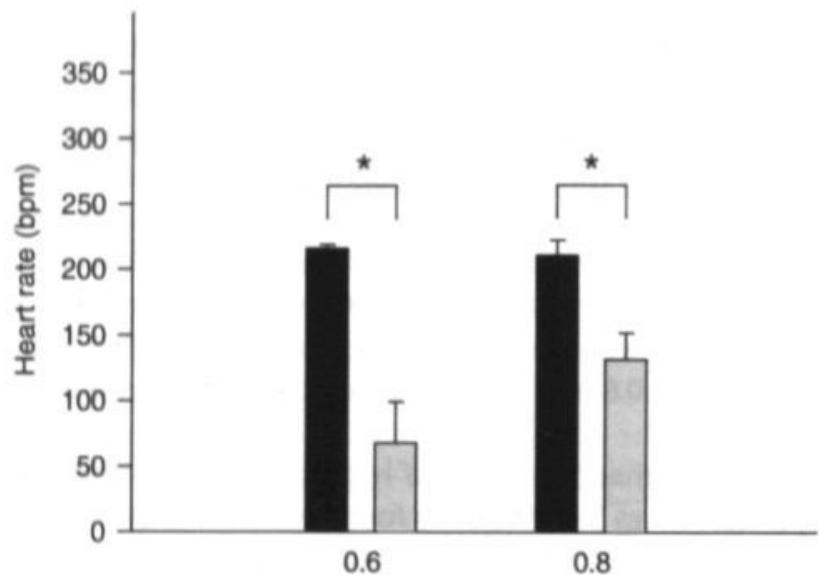

Figure 4B

Figure 4

Effect of 5 minutes of hypoxia on mean arterial pressure (A) and heart rate (B) at 0.6 and 0.8 foetal incubation time.

" $p<0.05$, vs. baseline; "\# $p<0.01,0.6$ vs. 0.8 foetal incubation time

Mean arterial pressure under baseline conditions significantly increased from 11 $\pm 0.4 \mathrm{~mm} \mathrm{Hg}$ at 0.6 foetal incubation time to $21 \pm 0.6 \mathrm{~mm} \mathrm{Hg}$ at 0.8 foetal incubation time $(p<0.01)$. During acute hypoxia mean arterial pressure significantly decreased to $4.0 \pm 1.1 \mathrm{~mm} \mathrm{Hg}(p<0.05)$ at 0.6 and $11.8 \pm 2.8 \mathrm{~mm} \mathrm{Hg}(\mathrm{p}<0.05)$ at 0.8 foetal incubation time (Fig. $4 \mathrm{~A}$ ). Heart rate under baseline conditions was not significantly different between 0.6 and 0.8 foetal incubation time ( $216 \pm 3$ beats/ min and $210 \pm 14$ beats/min, respectively). During hypoxia heart rate significantly decreased to $68 \pm 31$ beats $/ \mathrm{min}(\mathrm{p}<0.05)$ at 0.6 and $132 \pm 20$ beats $/ \mathrm{min}(\mathrm{p}<0.05)$ at 0.8 foetal incubation time (Fig. 4B). 


\section{Effect of topically applied phentolamine}

Topical application of PHENT alone did not significantly alter baseline mesenteric arterial diameters at 0.6 and 0.8 foetal incubation time $(97 \pm 2.5 \%$ and $98 \pm 3.0 \%$, respectively). At 0.6 foetal incubation time, neither the hypoxia-associated vasoconstriction (to $89 \pm 4.0 \%$ of baseline) nor the vasodilation during reoxygenation (to $116 \pm 3.2 \%$ of baseline) were significantly affected by $\alpha$-adrenergic blockade. In contrast, at 0.8 foetal incubation time the vasoconstrictor response during hypoxia was significantly attenuated by $\alpha$-adrenergic blockade ( $p<0.01$, Fig. 5$)$. In the presence of PHENT acute hypoxia induced only a slight decrease in mesenteric arterial diameter (to $93 \pm 2.7 \%$ of baseline, $\mathrm{p}-0.05$ ). Subsequently, diameters increased to $117 \pm 3.3 \%$ above baseline ( $p<0.05$ ), and further increased to $122 \pm 2.7 \%$ ( $p<0.05)$ during reoxygenation.

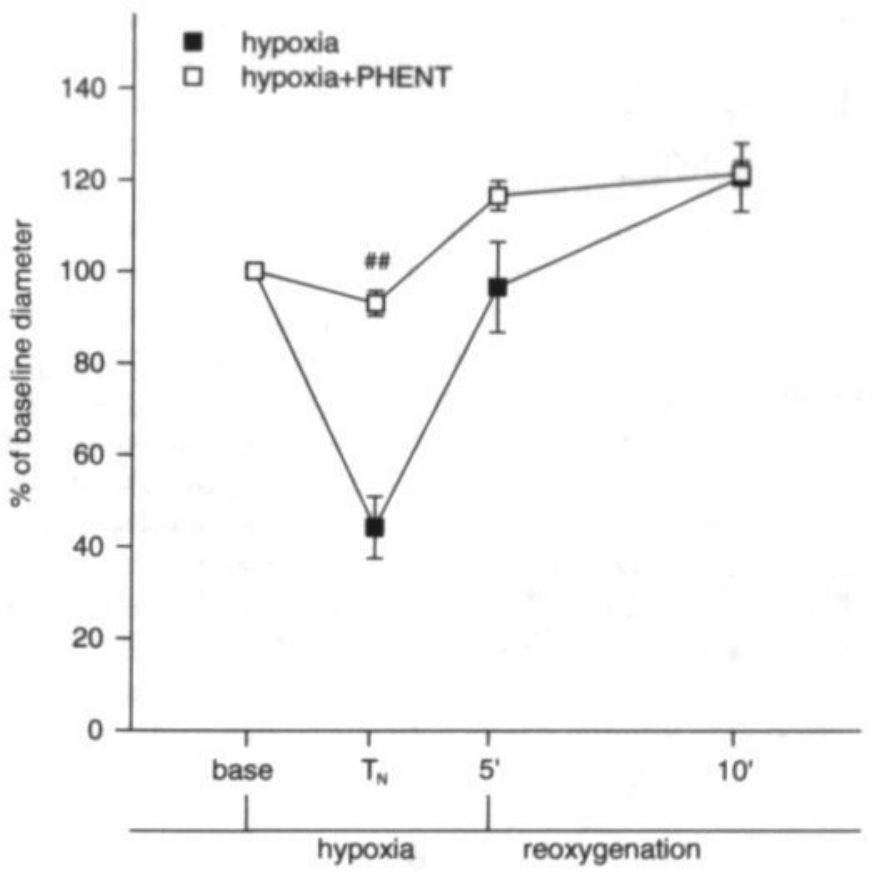

Figure 5

Effect of topically applied phentolamine on vasomotor responses during acute hypoxia and reoxygenation at 0.8 foetal incubation time. Alpha-adrenergic blockade significantly reduced the hypoxia-associated vasoconstriction.

es $p<0.01$, hypoxia in the presence of phentolamine vs. hypoxia alone 


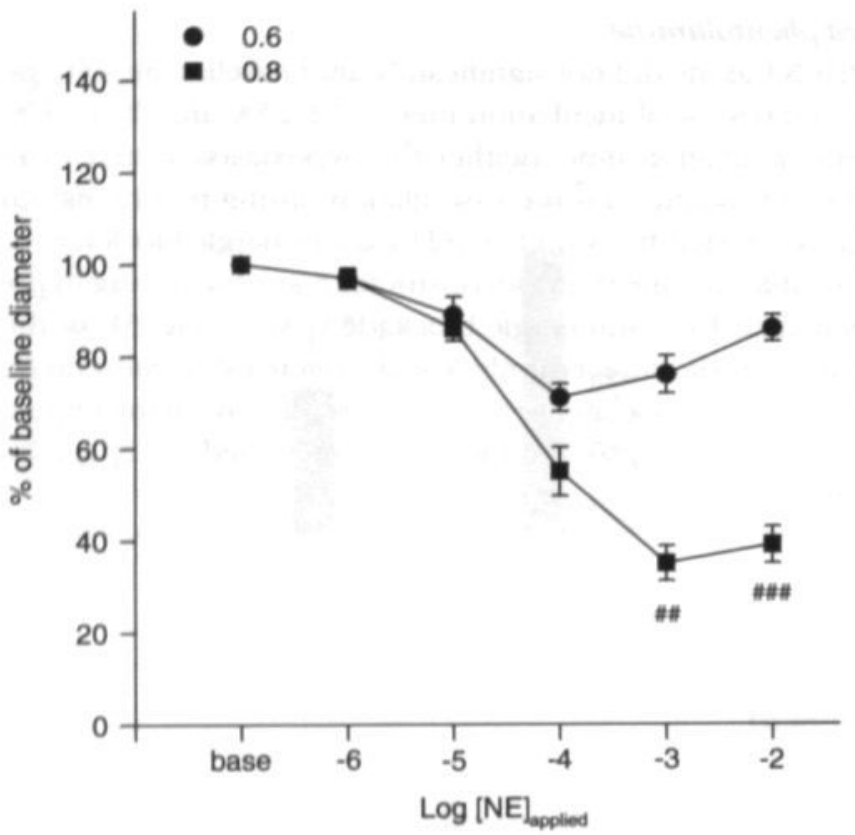

Figure 6

Effect of topically applied norepinephrine on mesenteric arterial diameter at 0.6 and 0.8 foetal incubation time. Maximal constriction to $\mathrm{NE}$ was significantly greater at 0.8 as compared with 0.6 incubation.

\#A, "\#\# $\mathrm{p}<0.01, \mathrm{p}<0.001,0.6$ vs. 0.8 foetal incubation time

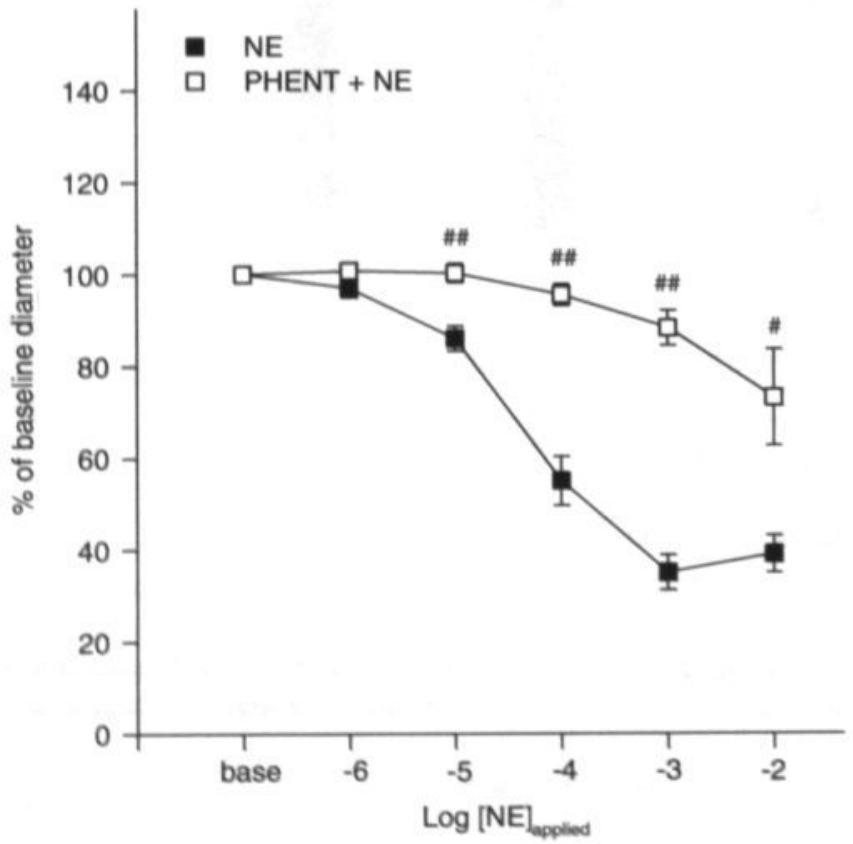

Figure 7

Efficacy of $\alpha$-adrenergic blockade with phentolamine. The vasoconstrictor response to $\mathrm{NE}$ was significantly reduced by phentolamine at 0.8 foetal incubation time.

\#, \#\# $\mathrm{p}<0.05, \mathrm{p}<0.01, \mathrm{NE}$ in the presence of phentolamine vs. NE alone 


\section{Effect of topically applied norepinephrine}

Cumulative application of increasing concentrations of NE caused a decrease in arterial diameter in both age groups (Fig. 6). Maximal vasoconstriction was observed after application of $10^{-4} \mathrm{M}\left(0.6\right.$ incubation) or $10^{-3} \mathrm{M}(0.8$ incubation $) \mathrm{NE}$. At these applied concentrations, arterial diameters significantly decreased to $71 \pm$ $3.0 \%(p<0.05)$ and $35 \pm 3.8 \%(p<0.01)$ of baseline at 0.6 and 0.8 foetal incubation time, respectively. The magnitude of maximal constriction to NE was approximately twofold larger at 0.8 as compared with 0.6 foetal incubation time ( $p<0.01$ ).

Topical administration of a $20 \mu \mathrm{l}$ aliquot of $10^{-3} \mathrm{M}$ phentolamine to the mesenteric arteries completely prevented the reduction in arterial diameter by norepinephrine at 0.6 foetal incubation time. At 0.8 foetal incubation time the concentration-response curve for norepinephrine shifted to the right in the presence of phentolamine, indicating a reduction in norepinephrine sensitivity (Fig. 7).

\section{Effect of topically applied acetylcholine}

Subsequent application of increasing concentrations of ACh to the same arteries resulted in a gradual vasodilation at both 0.6 and 0.8 foetal incubation time. Maximal arterial diameters were observed after application of $10^{-2} \mathrm{M} \mathrm{ACh}$, and were significantly different between 0.6 and 0.8 foetal incubation time $(63 \pm 3.5 \mu \mathrm{m}$ vs. 91 $\pm 2.3 \mu \mathrm{m}, \mathrm{p}<0.001$ ). However, expressed as percentage change from baseline, maximal levels of vasodilation were not significantly different between the two stages of foetal development (to $113 \pm 4.4 \%$ and $122 \pm 4.8 \%$ of baseline at 0.6 and 0.8 foetal incubation time, respectively; Fig. $8 \mathrm{~A}$ ).

Topical application of increasing doses of $\mathrm{ACh}$ under baseline conditions, i.e., without prior constriction with $\mathrm{NE}$, also caused a concentration-dependent dilation of the mesenteric arteries at 0.6 and 0.8 foetal incubation time. Maximal levels of vasodilation in both groups were not significantly different (to $125 \pm 2.6 \%$ and $133 \pm 5.4 \%$ of baseline at 0.6 and 0.8 foetal incubation time, respectively; Fig. $8 \mathrm{~B}$ ).

At both stages of foetal development, maximal levels of vasodilation after application of $10^{-2} \mathrm{M}$ ACh were similar in NE-constricted arteries and in arteries under baseline conditions. Furthermore, maximal diameters in response to topical application of ACh were not significantly different from maximal diameters observed during reoxygenation after 5 minutes of hypoxia. 


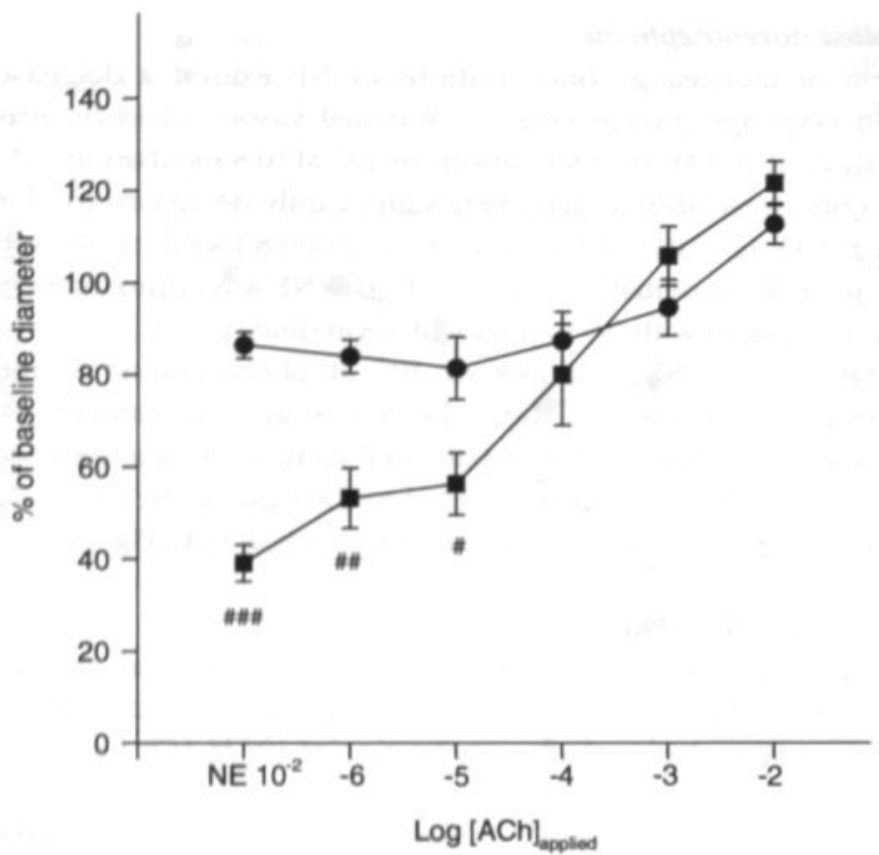

$\begin{array}{ll}- & 0.6 \\ \text { - } & 0.8\end{array}$

Figure 8A

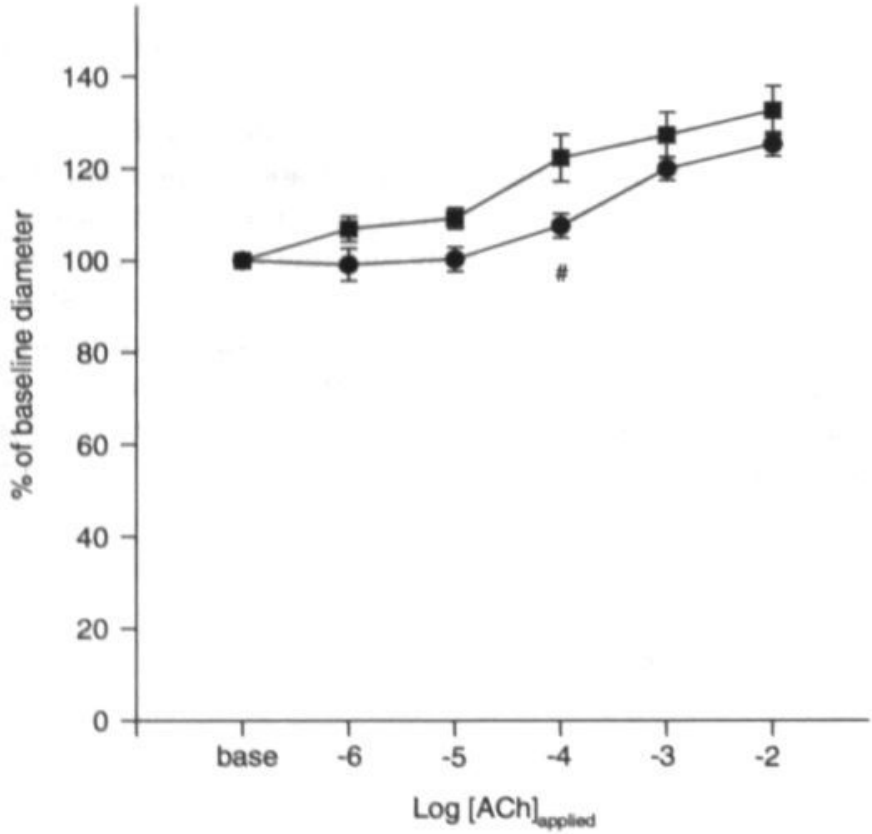

Figure 8B

Figure 8

Effect of topically applied acetylcholine on mesenteric arterial diameter at 0.6 and 0.8 foetal incubation time. ACh induced a significant increase in diameter above baseline level both in arteries subjected to NE-induced constriction (A) and in arteries under baseline conditions (B). Note that maximal vasodilation to ACh was similar at 0.6 and 0.8 foetal incubation time.

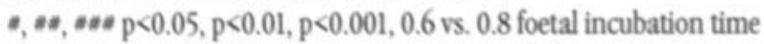




\section{DISCUSSION}

In order to obtain information with regard to the regulation of vascular tone in mesenteric arteries during foetal development, we designed a novel experimental setup for measurements of mesenteric arterial diameter in the intact chick foetus at different stages of foetal development. Between 0.6 and 0.8 of chick foetal incubation time, baseline luminal diameters of second order mesenteric arteries increased from $56 \mu \mathrm{m}$ to $75 \mu \mathrm{m}$. Since topical administration of the vasodilator substance acetylcholine induced an increase in arterial diameter of approximately $20 \%$ above baseline at both 0.6 and 0.8 foetal incubation time, it may be concluded that the mesenteric arteries already exhibit a degree of vascular tone under baseline conditions. Topical administration of the non-selective $\alpha$-adrenergic antagonist phentolamine did not affect baseline arterial diameter. This suggests that during the observed period of foetal development $\alpha$-adrenergic mechanisms are not involved in the establishment of baseline arterial tone at this level of the arterial tree.

The mesenteric arteries constricted in response to topically applied norepinephrine at both 0.6 and 0.8 foetal incubation time. Furthermore, this constriction was significantly attenuated in the presence of phentolamine. This indicates that functional $\alpha$-adrenergic pharmacomechanical coupling is present in the mesenteric arteries as early as 0.6 foetal incubation time. The maximal constrictor response to norepinephrine increased about twofold between 0.6 and 0.8 foetal incubation time. This may be due to developmental changes in the efficacy of the $\alpha$-adrenergic signal transduction pathway, amount of adrenoceptors as well as maturation of the vascular smooth muscle contractile apparatus. Interestingly, the mean arterial pressure increased about twofold during this period of development, from $11 \mathrm{~mm} \mathrm{Hg}$ at 0.6 to $21 \mathrm{~mm} \mathrm{Hg}$ at 0.8 foetal incubation time. Assuming that the perfusion pressure at the level of the second order mesenteric arteries also increased, the arteries at 0.8 foetal incubation time were subjected to a higher transmural pressure as compared with 0.6 foetal incubation time. Hence, in order to obtain the observed decreases in luminal diameter, the contractile force generated by the mesenteric arteries at 0.8 foetal incubation time must be substantially larger as compared with 0.6 foetal incubation time.

A 5-minute period of hypoxia induced a transient decrease in mesenteric arterial diameter at both stages of foetal development. The magnitude of the hypoxiaassociated vasoconstrictor response increased from a $13 \%$ reduction of arterial diameter at 0.6 incubation to a reduction of $56 \%$ at 0.8 foetal incubation time. At 0.6 foetal incubation time application of phentolamine did not significantly affect the vasoconstriction. This suggests that the constrictor response is not mediated by $\alpha$-adrenergic receptors at this stage of foetal development. In contrast, at 0.8 foetal incubation time the vasoconstriction during hypoxia was substantially attenuated in the presence of phentolamine. This suggests that it is at least in part mediated by $\alpha$-adrenoceptors at this stage of foetal development.

At 0.8 foetal incubation time the concentration-response curve for norepinephrine shifted to the right in the presence of phentolamine. This indicates a reduction in norepinephrine sensitivity, which is typical of a competitive antagonist such as phentolamine. Based on these results it cannot be ruled out that the 


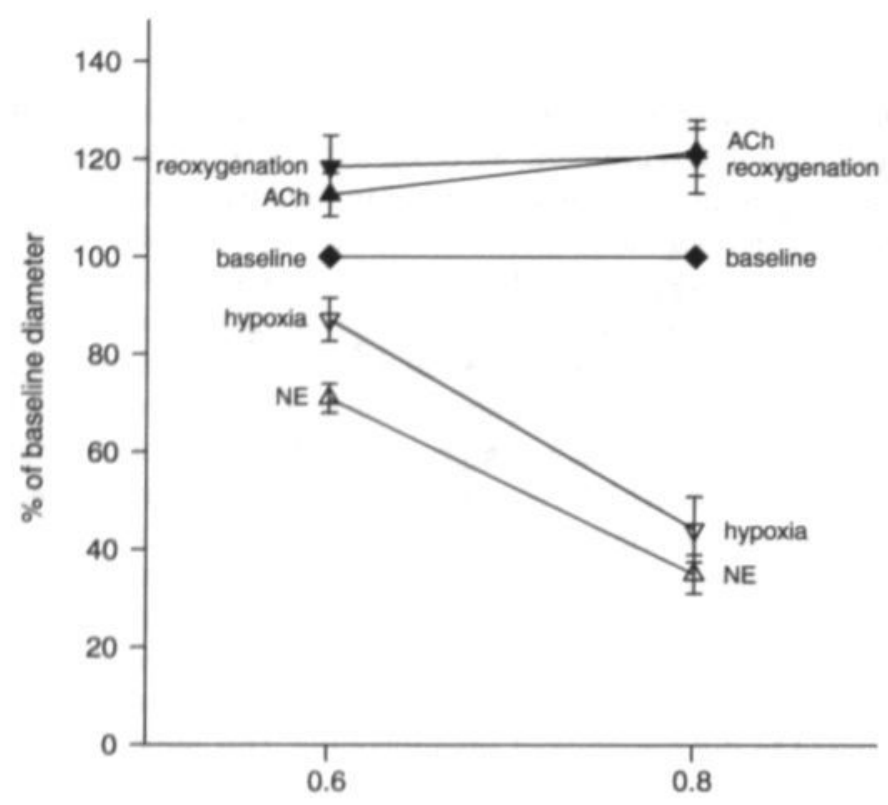

Foetal incubation time
Figure 9

Vasomotor responses of foetal mesenteric arteries at 0.6 and 0.8 foetal incubation time. Vasoconstrictor responses to acute hypoxia and to topically applied NE increased during foetal development, while vasodilator responses during reoxygenation and to topically applied ACh were similar at 0.6 and 0.8 foetal incubation time.

$7 \%$ constriction which remained during acute hypoxia in the presence of phentolamine is related to incomplete $\alpha$-adrenergic blockade at 0.8 foetal incubation time.

Central haemodynamic measurements showed that during acute hypoxia both heart rate and blood pressure decreased considerably at 0.6 as well as at 0.8 foetal incubation time. Assuming a reduction of perfusion pressure at the level of the mesenteric arteries, luminal diameter may passively decrease due to elastic recoil of the arteries subjected to a lower perfusion pressure. This may alternatively explain the observed small diameter reduction in the presence of phentolamine.

Finally, it may be argued that the difference in the vasoconstrictor response during acute hypoxia between 0.6 and 0.8 foetal incubation time is due to a difference in the level of hypoxia. Indeed, arterial $\mathrm{Po}_{2}$ during acute hypoxia is lower at 0.8 as compared with 0.6 foetal incubation time. However, arterial $\mathrm{Po}_{2}$ under normoxic conditions is also proportionally lower at 0.8 as compared with 0.6 foetal incubation time, thus the relative decrease in arterial $\mathrm{Po}_{2}$ is comparable. The mechanisms underlying the developmental change in arterial $\mathrm{PO}_{2}$ under normoxic conditions are beyond the scope of this article but are discussed in detail by Tazawa et al (14). Briefly, it has been demonstrated that during the third trimester of chick foetal incubation under normoxic conditions there is a gradual decrease in arterial $\mathrm{PO}_{2}$ and an increase in $\mathrm{PCO}_{2}$ which is in part metabolically compensated. According to these authors the changes in blood gas levels during late foetal development are related to a rise in metabolic rate of the foetus during this period of incubation. Oxygen concentration of the arterial blood, however, is maintained by concomitant increases in haematocrit and oxygen affinity of haemoglobin. Since it has been postulated that oxygen sensitive cells sense the oxygen concentration of the blood (9), it seems unlikely that the difference in the vasoconstrictor response 
during acute hypoxia between 0.6 and 0.8 foetal incubation time is due to the difference in arterial $\mathrm{Po}_{2}$.

Thus, the current study shows that foetal mesenteric arteries constrict in response to acute hypoxia, and indicates that the increase in magnitude of this vasoconstrictor response during $0.6-0.8$ of foetal development results from an increase in adrenergic constrictor capacity.

Interestingly, during the reoxygenation period the mesenteric arterial diameters increased above baseline levels, suggesting a hyperaemic response at both 0.6 and 0.8 foetal incubation time. The maximal arterial diameters obtained during the reoxygenation period were comparable to the levels of vasodilation obtained with topically applied $10^{-2} \mathrm{M}$ acetylcholine, a concentration sufficient to obtain maximal vasodilation. The vasodilator response actually started during the hypoxic phase and progressed during the reoxygenation phase. Maximal vasoconstriction during the 5-minute period of hypoxia was observed at approximately 100 seconds from the start of hypoxia, and lasted for about 30 seconds. By the end of the 5-minute hypoxic period arterial diameters returned almost to baseline level. In contrast, the vasoconstriction observed after topical application of norepinephrine under normoxic conditions lasted as long as 30 minutes (data not shown). Since acute hypoxia in the chick foetus is associated with a rise in plasma levels of norepinephrine (15), it is interesting to observe a vasodilator response already during the hypoxic phase. This suggests that secondary to the initial constriction in response to hypoxia a potent vasodilator substance is released locally.

In conclusion, the chick foetal mesenteric vasculature is a readily accessible vascular bed for studying the development of vasomotor control in small resistance-type arteries. In the current study we showed that $\alpha$-adrenergic pharmacomechanical coupling is already present in chick foetal mesenteric arteries as early as 0.6 foetal incubation time. The ability of mesenteric arteries to constrict in response to both physiological and pharmacological stimuli increases between 0.6 and 0.8 foetal incubation time. In contrast, vasodilator responses of the mesenteric arteries remain constant during this period of foetal development. This may suggest that the maturation of vasodilator mechanisms precedes that of vasoconstrictor mechanisms during foetal development. 


\section{References}

1. Crissinger K.D. Regulation of hemodynamics and oxygenation in developing intestine: insight into the pathogenesis of necrotizing enterocolitis. Acta Paediatr Suppl 1994; 396: 8-10.

2. Nowicki P.T. and Nankervis C.A. The role of the circulation in the pathogenesis of necrotizing enterocolitis. Clin Perinatol 1994; 21: 219-233.

3. Jensen A. and Berger R. Foetal circulatory responses to oxygen lack.J Dev Physiol 1991; 16: 181-207.

4. Mulder A.L.M., van Golde J.C., Prinzen F.W., and Blanco C.E. Cardiac output distribution in response to hypoxia in the chick embryo in the second half of the incubation time. JPhysiol 1998; 508: 281-287.

5. Jensen $\mathbf{A}$. and Lang $\mathrm{U}$. Foetal circulatory responses to arrest of uterine blood flow in sheep: effects of chemical sympathectomy. J Dev Physiol 1992; 17: 75-86.

6. Reuss M.L., Parer J.T., Harris J.L., and Krueger T.R. Hemodynamic effects of alpha-adrenergic blockade during hypoxia in foetal sheep. Am J Obstet Gynecol 1982; 142: 410-415.

7. Le Noble J.L., Struyker-Boudier H.A., and Smits J.F. Differential effects of general anesthetics on regional vasoconstrictor responses in the rat. Anch Int Pharmacodyn Ther 1987; 289: 82-92.

8. Iwamoto H.S., Kaufman T., Keil L.C., and Rudolph A.M. Responses to acute hypoxemia in foetal sheep at 0.6-0.7 gestation. Am J Physiol 1989; 256: H613-H620.

9. Semenza G.L. Perspectives on oxygen sensing Cell 1999; 98: 281-284.

10. Hamburger $\mathrm{V}$. and Hamilton H.L. A series of normal stages in the development of the chick embryo. JMorphol 1951; 88: 4998.

11. Slaaf D.W., Tangelder G.J., Reneman R.S., Jager K., and Bollinger A. A versatile incident illuminator for intravital microscopy. Int J Microcinc Clin Exp 1987; 6: 391-397.

12. Höchel J., Akiyama R., Masuko T., Pearson J.T., Nichelmann M., and Tazawa H. Development of heart rate irregularities in chick embryos. Am J Physiol 1999; 275: H527-H533.

13. Le Noble J.L., Tangelder G.J., Slaaf D.W., van Essen H., Reneman R.S., and Struyker-Boudier H.A.A functional morphometric study of the cremaster muscle microcirculation in young spontaneously hypertensive rats. J Hypertens 1990; 8: $741-748$.

14. Tazawa H., Visschedijk A.H.J., Wittmann J., and Piiper J. Gas exchange, blood gases and acid-base status in the chick before, during and after hatching. Respir Physiol 1983; 53: 173-185.

15. Wittmann J. and Prechtl J. Respiratory function of catecholamines during the late period of avian development. Respir Physiol 1991; 83: 375-386. 


\section{CHAPTER 7}

Chronic Foetal Hypoxia Alters Haemodynamic Control

Truly, birth marks not a beginning but a stage in life's journey. (J.W. Ballantyne, Manual of Antenatal Pathology and Hygiene, 1902) 


\section{INTRODUCTION}

During embryonic development, blood vessels arise through the processes of vasculogenesis, angiogenesis, and arteriogenesis. The primitive vessels develop into arteries and veins after recruitment of vascular smooth muscle cells and commencement of perfusion. Molecular mechanisms controlling the morphogenic transition of primitive blood vessels into a mature vascular network are rapidly being unraveled (1). However, little is known on the acquisition of vasomotor properties. It is still unknown when these newly formed arteries attain the ability to regulate local vascular tone in response to the oxygen demand of the individual organs, when central nervous coordination over the different vascular beds is instituted in order to regulate blood pressure, and which signals guide the maturation of this complex regulation.

According to the foetal programming hypothesis, aberrant development of the mechanisms which regulate vascular tone lies at the origin of cardiovascular diseases in later life, for example essential hypertension. It is speculated that deprivation of oxygen availability during foetal development, which is associated with intrauterine growth retardation, induces permanent alterations in the functional behaviour of arteries, thereby increasing peripheral vascular resistance and programming for hypertension. This hypothesis is based on epidemiologic relationships between low birth weight and development of cardiovascular disease in adulthood (2).

It is well established that hypoxia triggers the production and release of vascular endothelial growth factor (VEGF) and adenosine, which stimulate angiogenesis and arteriogenesis, respectively $(1,3,4)$. Both factors also exert vasodilator effects (5). These observations support the idea that chronic hypoxia may alter arterial network design and local regulation favouring a reduction in peripheral vascular resistance, rather than the hypothesised rise in resistance. Such an uncompensated decrease in vascular resistance might result in a drop in central blood pressure. In order to retain blood pressure, it may be postulated that foetal hypoxia interferes with central autonomic nervous control of the vasculature. Recent experimental evidence indeed suggests that hypoxia or angiogenic factors related to hypoxia may exert neurotrophic effects or modulate nervous function $(6-8)$.

It is clear that oxygen availability is a critical determinant of the long-term design of the vascular system. The objective of this study was to investigate in vivo the role of oxygen on the long-term establishment of local vascular tone as well as on the regulation of central haemodynamics. To this end, we studied the effects of prolonged exposure to a physiologically relevant level of hypoxia on $\alpha$-adrenoceptor mediated vasoconstriction and nitric oxide-dependent dilation, as well as the functional maturation of the sympathetic nervous system, in combination with measurements of arterial pressure and heart rate in near-term chick foetuses. 


\section{MATERIALS AND METHODS}

Experimental procedures were in accordance with the Dutch law on the use of laboratory animals. Fertile Lohman selected White Leghorn eggs were incubated at $37{ }^{\circ} \mathrm{C}$ and a relative air humidity of $60 \%$. Experiments were conducted in chick foetuses at day 19 of the 21-day incubation period, corresponding to 0.9 of total foetal incubation time. From the first day of incubation onward, foetuses in the normoxic $(\mathrm{N})$ group were incubated at $21 \% \mathrm{O}_{2}$, whereas foetuses in the chronic hypoxic $(\mathrm{CH})$ group were incubated at $15 \% \mathrm{O}_{2}$. Survival rate over the 19-day incubation period in the $\mathrm{CH}$ group was lower as compared with the $\mathrm{N}$ group $(51.4 \%$ vs. 72.5\%).

To assess the effect of chronic hypoxia on foetal arterial oxygen levels and erythropoiesis, blood gas values and haematocrit were determined under baseline $\left(21 \% \mathrm{O}_{2}\right)$ conditions in a separate series of normoxic and chronic hypoxic foetuses, as previously described (9). Body weights were measured to determine the effect of chronic hypoxia on foetal growth.

\section{Surgical preparation}

All surgical preparations were performed in a clinical infant incubator $\left(21 \% \mathrm{O}_{2}\right)$, as described in detail elsewhere (9). In short, the egg was opened at the blunt end containing the air cell, and after penetrating the chorioallantoic membrane (CAM), the foetus was stabilised by means of two sutures through connective tissue of the abdominal wall. For assessment of vasomotor responses, the omphalocele was centered at the level of the CAM, opened by careful blunt dissection, and the intestine was exposed. A segment of the ileum with its mesentery was placed on a $1 \mathrm{~cm}$ piece of cotton tape which was attached to the egg shell. Subsequently, the egg was transferred to a single-gg chamber where temperature $\left(37^{\circ} \mathrm{C}\right)$ and air flow $(21 \%$ $\mathrm{O}_{2}, 4 \mathrm{l} / \mathrm{min}$ ) were controlled, and placed in an intravital microscope setup (9).

\section{Intravital videomicroscopy}

The intestine was epi-illuminated in vivo with a $75 \mathrm{~W}$ Xenon lamp. Second order mesenteric resistance arteries were visualised using an intravital microscope, equipped with a $20 x$ long working distance objective, and a Ploemopak illuminator $2.1(1.25 \mathrm{x})$ with a polarizer-analyzer cube (Leitz), resulting in a $25 \mathrm{x}$ magnification at the camera plane. Images were projected onto a CCD camera (Hamamatsu Photonics, Hamamatsu, Japan), displayed on a monitor screen, and stored on videotape. Final resolution was $1 \mu \mathrm{m}$.

Luminal arterial diameters were analysed off-line using an image-shearing device (IPM, model 908, San Diego, CA). In the course of an experiment, all measurements were performed at the same site of the mesenteric resistance artery. Changes in diameter are presented as percentage of baseline diameter, with baseline being $100 \%$.

\section{Experimental protocol}

To investigate the effect of chronic hypoxia on the establishment of $\alpha$-adrenoceptor mediated vasoconstriction and dilation, we measured the changes in mesenteric arte- 
rial diameter in response to topically applied norepinephrine (NE) and additional acetylcholine (ACh) in normoxic and chronic hypoxic foetuses. After an equilibration period of 15 minutes following surgical preparation, a 2-minute baseline recording was made. A cumulative log molar concentration-response curve was constructed by sequential application of $20 \mu \mathrm{l}$ aliquots of increasing concentrations $\left(10^{6}-10^{-2} \mathrm{M}\right)$ of NE to the mesentery, and recording of the mesenteric artery image for 2 minutes following each application. The vasodilator capacity of the NE-constricted arteries was subsequently determined in a similar way by measuring the changes in arterial diameters in response to application of $20 \mu \mathrm{l}$ aliquots of $10^{-6}-10^{-2} \mathrm{M} \mathrm{ACh}$.

The effect of chronic hypoxia on the functional maturation of perivascular sympathetic nerves was assessed by measuring changes in mesenteric arterial diameter in response to topically applied tyramine (Tyr, $10^{-6}-10^{-2} \mathrm{M}$ ). Tyramine releases norepinephrine from perivascular sympathetic nerve endings, and does not affect presynaptic reuptake $(10,11)$.

In addition, changes in mesenteric arterial diameter as well as in central haemodynamic parameters were determined in response to a short period of severe hypoxia. After equilibration and baseline recording, acute hypoxia was induced by replacing the ambient air $\left(21 \% \mathrm{O}_{2}\right)$ in the egg chamber by $100 \% \mathrm{~N}_{2}$ (4 $\mathrm{l} / \mathrm{min}$ ) for 5 minutes. Reoxygenation was achieved by replacing the $\mathrm{N}_{2}$ with ambient air. During this period one mesenteric artery was continuously kept in focus and recorded.

Mean arterial pressure and heart rate under baseline conditions as well as during acute hypoxia were measured according to a previously described method (9). In short, a $10-\mathrm{cm}$-long nylon catheter (internal diameter $0.5 \mathrm{~mm}$ ) which was filled with $0.9 \% \mathrm{NaCl}$ was inserted into one of the two branches of the chorioallantoic artery with its tip pointing upstream. The free end of the catheter was connected to a pressure transducer (Baxter Uniflow, Baxter B.V., Uden, the Netherlands) which was placed at the same height as the egg. Pressure signals were recorded on a computer using a data acquisition system and the heart rate was calculated.

\section{Drugs and solutions}

Norepinephrine (1-artenolol-bitrate, NE), acetylcholine (ACh) and tyramine (Tyr) were obtained from Sigma Chemical Co (St. Louis, MO). Drugs were dissolved in HEPES buffered Krebs with the following composition (in $\mathrm{mM}$ ): $\mathrm{NaCl} 143.3, \mathrm{KCl} 4.7$, $\mathrm{MgSO}_{4} 1.2, \mathrm{KH}_{2} \mathrm{PO}_{4} 1.2, \mathrm{CaCl}_{2} 2.5$, glucose 5.6, HEPES 15. The pH of the buffer was adjusted to 7.4. Temperature at the time of administration was $37^{\circ} \mathrm{C}$.

\section{Data analysis}

Data are expressed as mean \pm SEM. The term $n$ refers to the number of foetuses; observations were made in one artery per foetus. Statistical comparisons between groups were made using the Mann-Whitney U test. Statistical comparisons within groups were made using the Wilcoxon signed ranks test. Statistical significance was defined as $\mathrm{p}<0.05$. 


\section{RESULTS}

\section{Baseline characteristics}

The effects of chronic hypoxia $\left(15 \% \mathrm{O}_{2}\right)$ on foetal characteristics at 0.9 incubation time, as measured under baseline $\left(21 \% \mathrm{O}_{2}\right)$ conditions, are shown in Table 1 . Chronic hypoxic foetuses had a significantly lower body weight ( $21.9 \pm 0.49$ vs. 24.7 $\pm 0.40 \mathrm{~g}, \mathrm{p}<0.001)$, and higher haematocrit $(0.34 \pm 0.014$ vs. $0.30 \pm 0.010$, p<0.05) as compared with normoxic foetuses. The reduced survival rate, lower body weight, and higher haematocrit in chronic hypoxic foetuses are indicative of prolonged reduction in oxygen availability to the growing foetus, which justifies the experimental protocol used. Arterial $\mathrm{Po}_{2}$ levels under baseline (normoxic) conditions were similar in both groups (6.3 \pm 0.96 and $5.8 \pm 0.46 \mathrm{kPa})$, whereas $\mathrm{PcO}_{2}$ was lower in the $\mathrm{CH}$ group $(3.9 \pm 0.26$ vs. $4.8 \pm 0.34 \mathrm{kPa}$, $\mathrm{p}<0.05)$.

Table 1. Effects of chronic hypoxia on baseline foetal characteristics at 0.9 foetal incubation time

\begin{tabular}{lll}
\hline Variable & Normoxic $(n)$ & Chronic hypoxic $(n)$ \\
\hline Body weight $(\mathrm{g})$ & $24.7 \pm 0.40(21)$ & $21.9 \pm 0.49^{* * *}(22)$ \\
Haematocrit & $0.30 \pm 0.01(17)$ & $0.34 \pm 0.014^{*}(7)$ \\
Blood gas values & $(7)$ & $(7)$ \\
$\quad \mathrm{PO}_{2}(\mathrm{kPa})$ & $5.78 \pm 0.46$ & $6.30 \pm 0.96$ \\
$\mathrm{PCO}_{2}(\mathrm{kPa})$ & $4.83 \pm 0.34$ & $3.93 \pm 0.26^{*}$ \\
$\mathrm{pH}$ & $7.48 \pm 0.04$ & $7.47 \pm 0.03$ \\
\hline
\end{tabular}

$\because * * 0<0.05, p<0.001$, chronic hypoxic vs. normoxic foetuses

\section{Effect of topically applied norepinephrine and acetylcholine}

Absolute baseline diameters were similar in the $\mathrm{CH}$ and $\mathrm{N}$ group ( $89 \pm 5.1$ and 98 $\pm 5.0 \mu \mathrm{m}, n=7$ and $n=9$ ). Cumulative application of increasing concentrations of norepinephrine (NE) caused a decrease in arterial diameter in the $\mathrm{CH}$ and $\mathrm{N}$ group (Fig. 1A). Maximal constrictor response, obtained at a concentration of $10^{-3} \mathrm{M} \mathrm{NE}$, was similar in both groups (to $34 \pm 3.6 \%$ and $29 \pm 2.0 \%$ of baseline).

Subsequent application of increasing concentrations of acetylcholine (ACh) to the NE-constricted arteries resulted in a gradual dilation in the $\mathrm{CH}$ and $\mathrm{N}$ group. Mesenteric arteries in the $\mathrm{CH}$ group dilated to a significantly larger extent in response to $10^{-4} \mathrm{M} \mathrm{ACh}$ as compared with the $\mathrm{N}$ group (to $88 \pm 12.0 \%$ vs. $67 \pm 9.1 \%$ of baseline, p<0.05), indicating a shift to the left of the dose-response curve for ACh (Fig. 1B). Maximal dilator response, attained at a concentration of $10^{-2} \mathrm{M} \mathrm{ACh}$, was also significantly greater in the $\mathrm{CH}$ group as compared with the $\mathrm{N}$ group (to $118 \pm 4.9 \%$ vs. $105 \pm 2.4 \%$ of baseline, p<0.05; Fig. 2 ). However, absolute maximal diameters were similar in both groups $(104 \pm 5.0$ and $104 \pm 4.9 \mu \mathrm{m})$. 

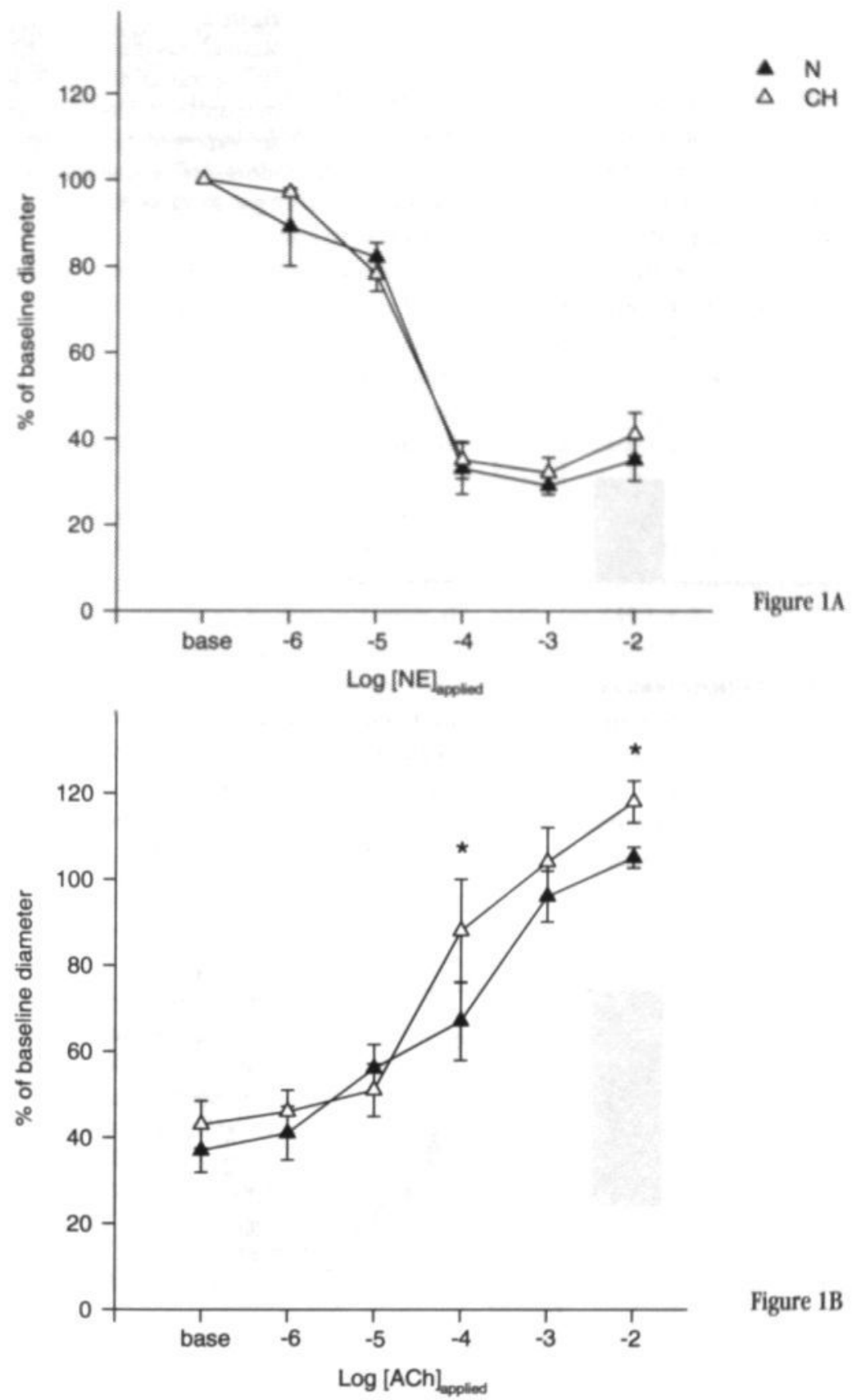

\section{Figure 1}

Effect of topically applied norepinephrine (A) and acetylcholine (B) on mesenteric arterial diameter in normoxic $(\mathrm{N})$ and chronic hypoxic $(\mathrm{CH})$ foetuses at 0.9 foetal incubation time. Maximal constriction to $\mathrm{NE}$ was similar in both groups. Subsequently, acetylcholine was applied on the NE-preconstricted mesenteric arteries. Sensitivity to $\mathrm{ACh}$ was higher in $\mathrm{CH}$ as compared with $\mathrm{N}$ foetuses, as indicated by the left-shift of the dose-response curve. Data are expressed relative to baseline diameter (100\%).

${ }^{*} \mathrm{P}<0.05$, CH vs. N group 


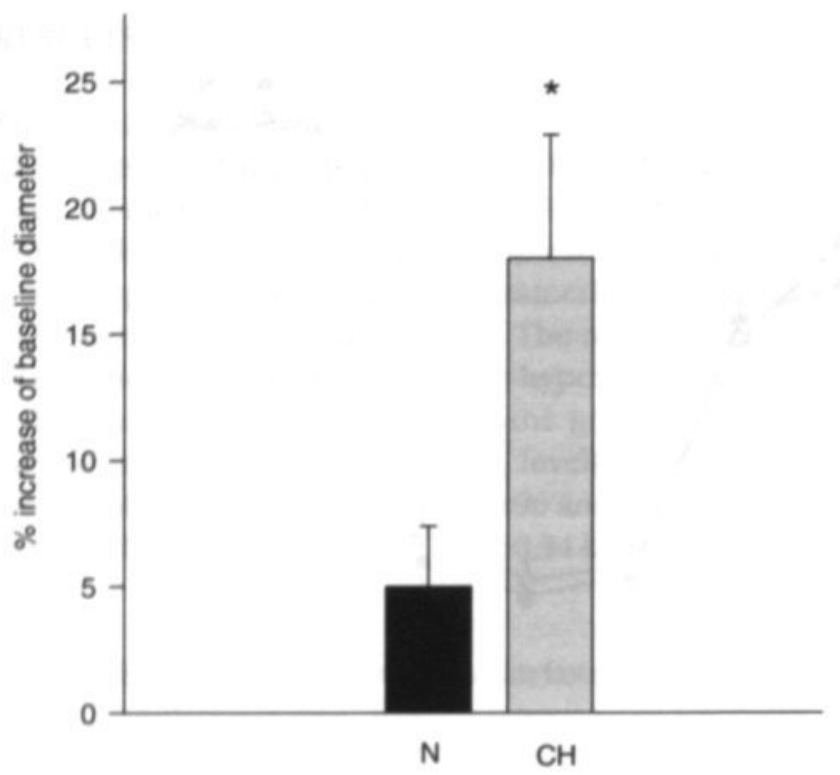

Figure 2

Maximal vasodilation to ACh $10^{-2} \mathrm{M}$ was greater in $\mathrm{CH}$ as compared with $\mathrm{N}$ foetuses. Data are expressed as percentage increase of baseline diameter. * $\mathrm{p}<0.05, \mathrm{CH}$ vs. N group

\section{Perivascular sympathetic nerves}

Topical administration of $20 \mu \mathrm{l}$ aliquots of increasing concentrations of tyramine (Tyr) induced a reduction in mesenteric arterial diameter in both the $\mathrm{CH}$ and $\mathrm{N}$ (both $n=8$, data not shown). Maximal constrictor response, attained at a concentration of $10^{-3} \mathrm{M} \mathrm{Tyr}$, was significantly greater in the $\mathrm{CH}$ compared with the $\mathrm{N}$ group ( $37 \pm 3.6 \%$ vs. $19 \pm 5.2 \%$ decrease of baseline, p<0.01; Fig. 3 ).

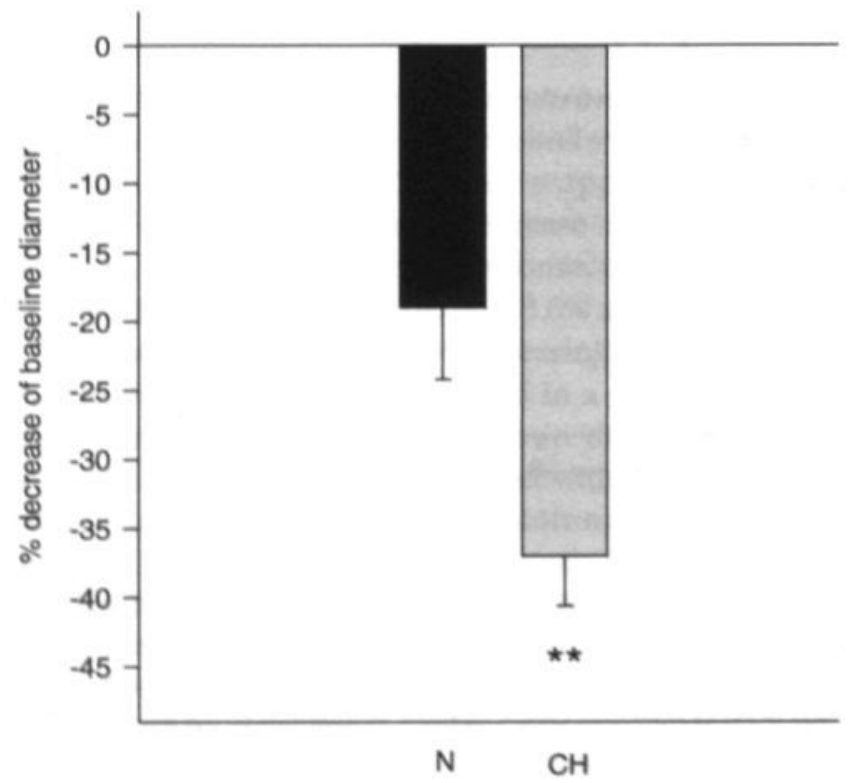

Figure 3

Effect of topically applied tyramine on mesenteric arterial diameter at 0.9 foetal incubation time. Maximal constriction to Tyr $10^{-3} \mathrm{M}$ was larger in $\mathrm{CH}$ as compared with $\mathrm{N}$ foetuses. Data are expressed as percentage decrease of baseline diameter.

** p<0.01, CH vs. N group 


\section{Effect of acute hypoxia}

Absolute baseline diameters were similar in the $\mathrm{CH}$ and $\mathrm{N}$ group $(94 \pm 4.8$ and 95 $\pm 4.5 \mu \mathrm{m}, n=9$ and $n-10$ ). The vasomotor responses of the mesenteric resistance arteries to acute hypoxia $\left(0 \% \mathrm{O}_{2}\right)$ and subsequent reoxygenation $\left(21 \% \mathrm{O}_{2}\right)$ were similar in the $\mathrm{CH}$ and $\mathrm{N}$ group, in magnitude as well as in time course (Fig. 4). In both groups, a significant and sustained reduction in arterial diameter was observed during 5 minutes of acute hypoxia (to $42 \pm 2.5 \%$ and $46 \pm 5.3 \%$ of baseline, p<0.01), which returned to baseline during reoxygenation.

Mean arterial pressure under baseline conditions was similar in the $\mathrm{CH}$ and $\mathrm{N}$ group ( $27 \pm 2.2$ and $25 \pm 1.4 \mathrm{~mm} \mathrm{Hg} ; n=8$ and $n=9$, Fig. 5A). However, during acute hypoxia, arterial pressure significantly decreased in the $\mathrm{CH}$ group (to $22 \pm 1.8 \mathrm{~mm}$ $\mathrm{Hg}$, p $<0.05)$, whereas it remained constant in the $\mathrm{N}$ group $(25 \pm 0.9 \mathrm{~mm} \mathrm{Hg})$. During reoxygenation arterial pressure significantly increased above baseline in the $\mathrm{CH}$ and $\mathrm{N}$ group (to $31 \pm 2.2$ and $35 \pm 1.7 \mathrm{~mm} \mathrm{Hg}, \mathrm{p}<0.05$ ). This increase was greater in the $\mathrm{N}$ group ( $<<0.01$ ). Baseline heart rate was also similar in the $\mathrm{CH}$ and $\mathrm{N}$ group ( $205 \pm 6$ and $199 \pm 9$ beats/min; Fig. 5B). Although acute hypoxia induced a significant fall in heart rate in both groups ( $<<0.01$ ), heart remained at a higher level in the $\mathrm{CH}$ group as compared with the $\mathrm{N}$ group $(132 \pm 3$ vs. $117 \pm 6$ beats $/ \mathrm{min}$, p 0.05 ). During reoxygenation heart rate returned to baseline in the $\mathrm{N}$ group (185 \pm 8 beats/min), but only partially recovered in the $\mathrm{CH}$ group ( $172 \pm 3$ beats $/ \mathrm{min}$, p<0.05).

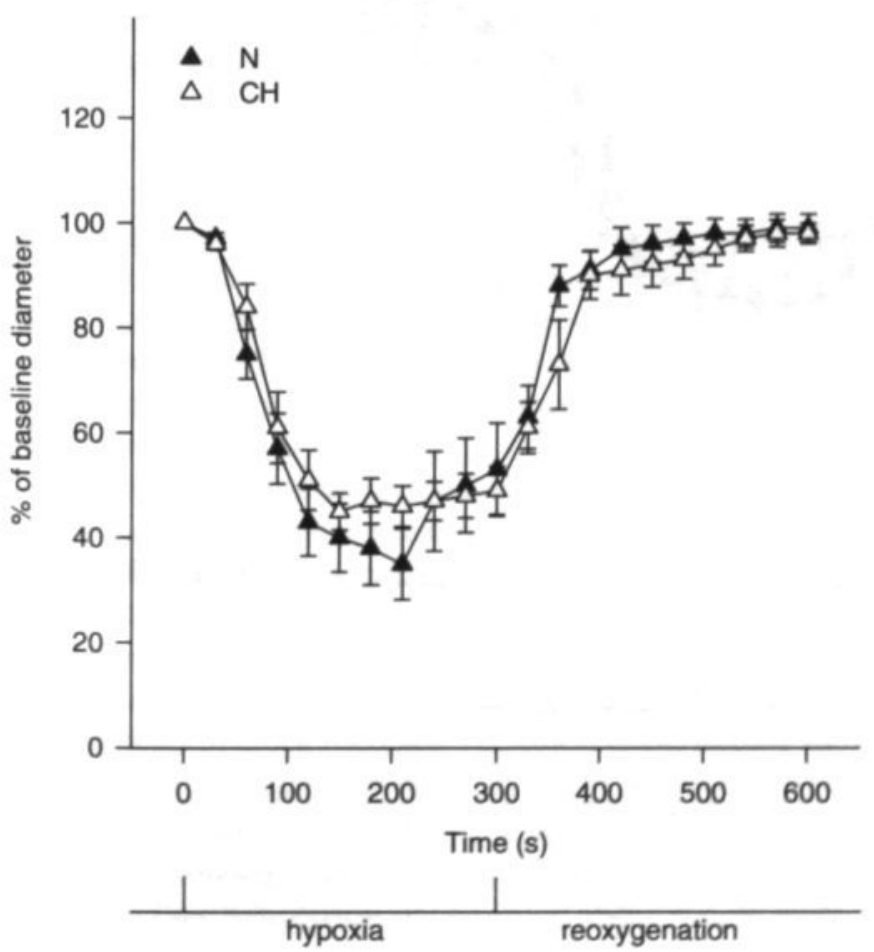

Figure 4

Effect of 5 minutes of severe hypoxia $\left(0 \% \mathrm{O}_{2}\right)$ followed by 5 minutes of reoxygenation $\left(21 \% \mathrm{O}_{2}\right)$ on mesenteric arterial diameter at 0.9 foetal incubation time. Time course and magnitude of the arterial response (sustained constriction during acute hypoxia, dilation to baseline during reoxygenation) was similar in $\mathrm{CH}$ and $\mathrm{N}$ foetuses. Data are expressed relative to baseline diameter $(100 \%)$. 


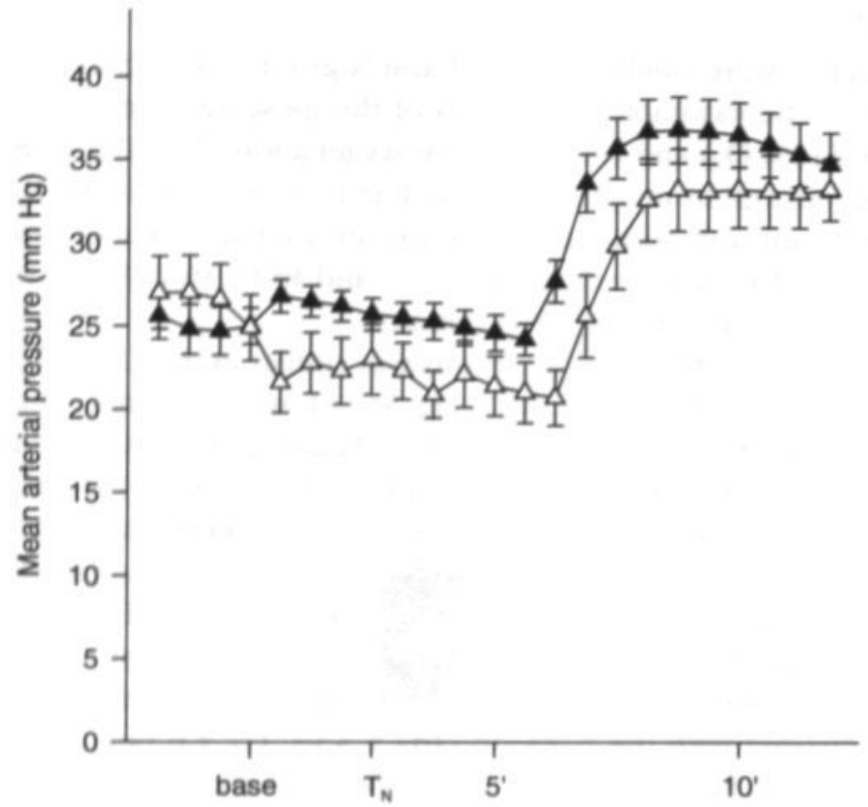

$\Delta \mathrm{N}$

$\triangle \mathrm{CH}$

Figure 5A

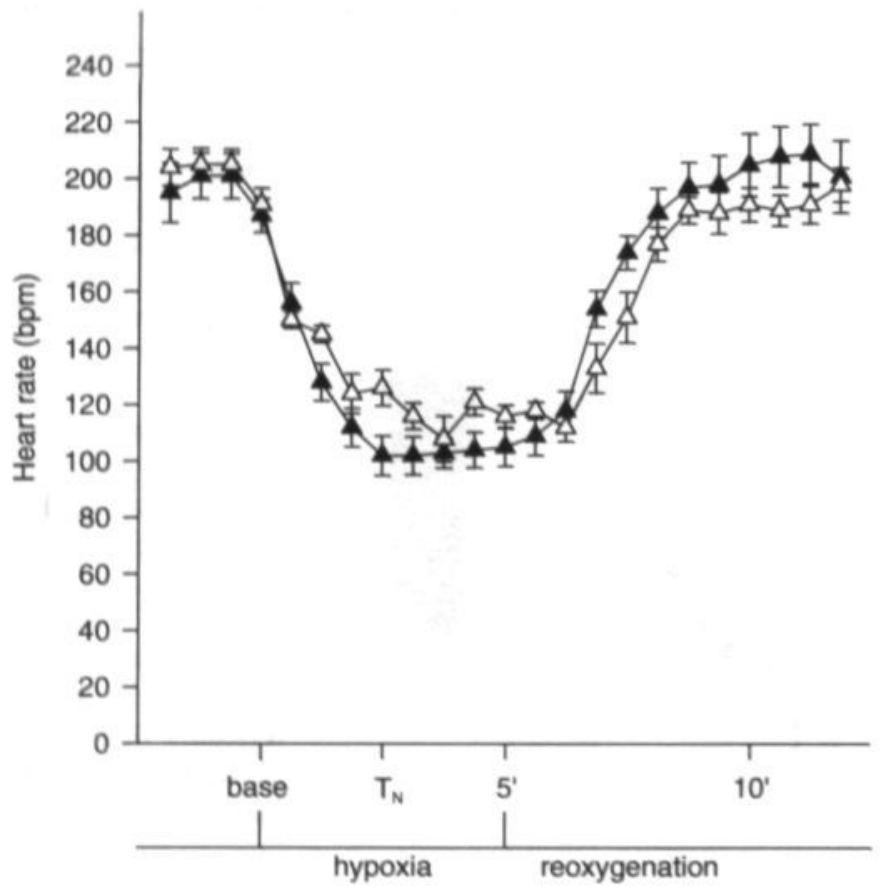

Figure 5B

\section{Figure 5}

Effect of 5 minutes of severe hypoxia on mean arterial pressure (A) and heart rate (B) at 0.9 foetal incubation time. Baseline blood pressure and heart rate was similar in $\mathrm{CH}$ and $\mathrm{N}$ foetuses. Acute hypoxia induced a fall in pressure and heart rate in $\mathrm{CH}$ foetuses. Although heart rate decreased to a similar degree, blood pressure was maintained in $\mathrm{N}$ foetuses. The increase in arterial pressure during reoxygenation was lower in $\mathrm{CH}$ as compared with $\mathrm{N}$ foetuses. 


\section{DISCUSSION}

This in vivo study conducted in intact, unanaesthetised near-term foetuses was initiated by the concept that reduced oxygen availability during foetal development may alter the functional behaviour of foetal arteries, and may influence blood pressure regulation. We demonstrated that chronic hypoxia results in enhanced contractile responsiveness to direct stimulation of perivascular sympathetic nerves in the mesenteric vascular bed of near-term foetuses. Since maximal responsiveness and sensitivity to norepinephrine were not altered, these data indicate that chronic hypoxia induces sympathetic hyperinnervation, which may lead to an increase in arterial tone. Chronic hypoxia was, however, not accompanied by an elevated systemic blood pressure level. These findings support the idea that hypoxia influences central autonomic nervous control over the individual vascular beds in order to maintain cardiovascular homeostasis.

Chronic hypoxia induced a twofold increase in the contractile response to tyramine at 0.9 foetal incubation time. In vivo measurement of arterial diameter reduction using tyramine is a sophisticated method enabling direct sympathetic nerve stimulation. The contractile response to tyramine has been demonstrated to reflect the total norepinephrine content of the perivascular sympathetic nerve endings (11). Since the $\alpha$-adrenergic sensitivity of the mesenteric arteries in our experiments was unaffected by chronic hypoxia, the greater constrictor response to tyramine of mesenteric arteries in chronic hypoxic as compared with normoxic foetuses is indicative of a higher norepinephrine content in the perivascular sympathetic nerves of chronic hypoxic foetuses. These data suggest that chronic hypoxia induces functional sympathetic hyperinnervation of peripheral resistance arteries.

Increased arterial tone after chronic hypoxia was suggested by the response to acetylcholine. Compared to baseline levels, the maximal vasodilator response to acetylcholine was larger in chronic hypoxic as compared with normoxic foetuses. However, in absolute values, arterial diameters during maximal dilation were similar in both groups. Since we have previously shown that acetylcholine at a concentration of $10^{-2} \mathrm{M}$ increases arterial diameter to a maximal extent (9), these data indicate that chronic hypoxia increases mesenteric arterial tone under resting conditions.

The increase in arterial tone at 0.9 foetal incubation time was not reflected in central haemodynamics, since mean arterial pressure and heart rate were similar in chronic hypoxic and normoxic foetuses. One explanation for this discrepancy may be that the total vascular resistance had not changed in chronic hypoxic foetuses. Two important determinants of vascular resistance are vascular tone and arterial branching pattern. Chronic hypoxia has previously been shown to stimulate angiogenesis in chick foetuses. Perfusion experiments showed that chick foetuses incubated at an oxygen level of $15 \%$ had a reduced whole body peripheral vascular resistance during maximal vasodilation, indicating that a hypoxia associated change in architecture of the vascular network was accompanied by a decrease in structural vascular resistance $(12,13)$. It may thus be suggested that the observed increase in arterial tone is compensated by a change in vascular network structure. 
Acute hypoxia is a stimulus for increasing efferent sympathetic nervous activity, and release of catecholamines from the adrenal gland (14). Our data show that in response to acute hypoxia, blood pressure in chronic hypoxic foetuses dropped, whereas blood pressure in normoxic foetuses remained at a constant level. However, the level of mesenteric arterial constriction was similar in both groups. Assuming that constriction in the splanchnic circulation reflects a generalised rise in peripheral vascular resistance (15), the blood pressure decrease in chronic hypoxic foetuses may have been caused by a reduction in cardiac output. Although it is known that acute hypoxia exerts a negative inotropic effect on the heart, studies demonstrating that chronic hypoxia potentiates this effect are lacking. To address this question further, detailed cardiac output measurements are necessary, but are currently beyond the possibilities of our foetal model.

At the cellular level, hypoxia increases the activity of the transcription factors hypoxia-inducible factor-1 (HIF-1) and HIF-2 (or EPAS-1). These transcription factors trigger the expression of genes which are held responsible for hypoxia associated-angiogenesis, including VEGF (16). Furthermore, HIF-1 and VEGF induce the expression of nitric oxide synthases in endothelial cells, promoting the production of nitric oxide (17-19). This may explain the enhanced sensitivity of the preconstricted mesenteric arteries for the muscarinic agonist acetylcholine, a known stimulator of endothelial nitric oxide production, which we observed in chronic hypoxic foetuses. VEGF also stimulates axonal outgrowth and cell survival in the peripheral nervous system (7), and via its coreceptor neuropilin-1, is implicated in sympathetic axon guidance (6). Knock-out mice lacking the hypoxiaresponse element in the VEGF promotor showed motor-neuron defects (8). However, it is yet not known whether sympathetic neurons are also affected.

Based on colocalisation of the expression of HIF-2 with the expression of tyrosine-hydroxylase, the rate limiting enzyme in the synthesis of norepinephrine, HIF-2 has been postulated to upregulate norepinephrine production (20). Mice lacking HIF-2 die at midgestation due to profoundly reduced catecholamine levels (21). The current study shows that chronic hypoxia increases the arterial responsiveness to tyramine. This is the first direct evidence obtained in vivo of enhanced norepinephrine production and secretion by perivascular sympathetic nerves after prolonged exposure to hypoxia. The increase in arterial tone after chronic hypoxia is the functional effect of this increase in sympathetic innervation.

Although hypoxia has been reported to alter the expression of $\alpha$-adrenergic receptor subtypes (22), the current study did not show any change in the sensitivity or maximal contractile responsiveness of the mesenteric arteries to norepinephrine after chronic hypoxia.

In conclusion, the present study indicates that reduced oxygen availability to the developing foetus results in sympathetic hyperinnervation, an increase in mesenteric arterial tone, but no change in blood pressure and heart rate in nearterm foetuses. We postulate that oxygen plays a critical role in the long-term orchestration of the haemodynamic regulation in pace with the structural design of the cardiovascular system. 


\section{References}

1. Carmeliet P. Mechanisms of angiogenesis and arteriogenesis. Nature Med 2000; 6:389-395.

2. Barker D.J.P., Osmond C., Golding J., Kuh D., and Wadsworth M.E.J. Growth in utero, blood pressure in childhood and adult life, and mortality from cardiovascular disease. BMJ 1989; 298: 564-567.

3. Grant M.B., Tarnuzzer R.W., Caballero S., Ozeck M.J., Davis M.L., Spoerri P.E., Feoktistov L., Biaggioni L., Shryock J.C., and Belardinelli L. Adenosine receptor activation induces vascular endothelial growth factor in human retinal endothelial cells. Cinc Res 1999, 85: 699-706.

4. Adair T.H., MontaniJ.P., Strick D.M., and Guyton A.C. Vascular development in chick embryos: a possible role for adenosine. Am J Physiol 1989, 256: H240-H246.

5. Zachary I. and Gliki G. Signal transduction mechanisms mediating biological actions of the vascular endothelial growth factor family. Candiovasc Res 2001; 49: 568-581.

6. Chen $\mathrm{H}$., He Z., Bagri A., and Tessier-Lavigne M. Semaphorin-neuropilin interactions underlying sympathetic axon responses to class III semaphorins. Neuron 1998; 21: 1283-1290.

7. Sondell M., Lundborg G., and Kanje M. Vascular endothelial growth factor has neurotrophic activity and stimulates axonal outgrowth, enhancing cell survival and Schwann cell proliferation in the peripheral nervous system. J Neurasci 1999, 19:5731-5740.

8. Oosthuyse B., Moons L., Storkebaum E., Beck H., Nuyens D., Brusselmans K., Van Dorpe J., Hellings P., Gorselink M., Heymans S., Theilmeier G., Dewerchin M., Laudenbach V., Vermylen P., Raat H., Acker T., Vleminckx V., Van den Bosch L., Cashman N., Fujisawa H., Drost M.R., Sciot R., Bruyninckx F., Hicklin D.J., Ince C., et al. Deletion of the hypoxia-response element in the vascular endothelial growth factor promotor causes motor neuron degeneration. Nat Genet 2001; 28: 131-138.

9. Rouwet E.V., De Mey J.G.R., Slaaf D.W., Heineman E., Ramsay G., and Le Noble F.A.C. Development of vasomotor responses in fetal mesenteric arteries. Am J Physiol Heart Circ Physiol 2000; 279: H1097-H1105.

10. Le Noble F.A.C., Ruijtenbeek K., Gommers S., De Mey J.G., and Blanco C.E. Contractile and relaxing reactivity in carotid and femoral arteries of chicken embryos. Am J Physiol Heart Circ Physiol 2000; 278: H1261-H1268.

11. Takauchi Y., Yamazaki T., and Akiyama T. Tyramine-induced endogenous noradrenaline efflux from in situ cardiac sympathetic nerve endings in cats. Acta Physiol Scand 2000; 168: 287-293.

12. Strick D.M., Waycaster R.L., Montani J.P., Gay W.J., and Adair T.H. Morphometric measurements of chorioallantoic membrane vascularity: effects of hypoxia and hyperoxia. Am J Physiol 1991; 260: H1385-H1389.

13. Adair T.H., Guyton A.C., Montani J.P., Lindsay H.L., and Stanek K.A. Whole body structural vascular adaptation to prolonged hypoxia in chick embryos. Am J Physiol 1987; 252: H1228-H1234.

14. Inoue M., Fujishiro N., and Imanaga I. Hypoxia and cyanide induce depolarization and catecholamine release in dispersed guinea-pig chromaffin cells. J Physiol 1998; 507: 807-818.

15. Jensen A. and Berger R. Regional distribution of cardiac output. In: Hanson M.A., Spencer J.A.D., Rodeck C.H., editors. Fetus and Neonate: Physiology and Clinical Applications. Volume 1: The Circulation. Cambridge: Cambridge University Press, 1993: 23-74.

16. Semenza G.L. Hypoxia-inducible factor 1: master regulator of $\mathrm{O}_{2}$ homeostasis. Curr Opin Genet Dev $1998 ; 8: 588-594$.

17. Kroll J. and Waltenberger J. VEGF-A induces expression of eNOS and iNOS in endothelial cells via VEGF receptor-2 (KDR). Biochem Biophys Res Commun 1998; 252: 743-746. 
18. Kroll J. and Waltenberger J. A novel function of VEGF receptor-2 (KDR): rapid release of nitric oxide in response to VEGF-A stimulation in endothelial cells. Biochem Biophys Res Commun 1999, 265: $636-639$.

19. Palmer L.A., Semenza G.L., Stoler M.H., and Johns R.A. Hypoxia induces type II NOS gene expression in pulmonary artery endothelial cells via HIF-1.Am J Physiol 1998; 274: L212-L219.

20. Favier J., Kempf H., Corvol P., and Gasc J.M. Cloning and expression pattern of EPAS1 in the chicken embryo. FEBS Lett 1999; 462: 1924.

21. Tian H., Hammer R.E., Matsumoto A.M., Russell D.W., and McKnight S.L. The hypoxia-responsive transcription factor EPAS1 is essential for catecholamine homeostasis and protection against heart failure during embryonic development. Genes Dev 1998; 12: 3320-3324.

22. Eckhart A.D., Zhu Z., Arendshorst W.J., and Faber J.E. Oxygen modulates alphalB-adrenergic receptor gene expression by arterial but not venous vascular smooth muscle. Am J Physiol 1996; 271: H1599H1608. 


\section{CHAPTER 8}

General Discussion 
122 
During foetal life an orderly sequence of developmental changes in intestinal structure and function prepares the gut for the demands of extrauterine life. Although the morphological differentiation of the intestinal epithelium is already completed halfway through foetal gestation, the functional development of the gut occurs at a much slower pace (Chapter 2). When an infant is born before term the transition from the intrauterine to the extrauterine environment is forced to occur ahead of time, which requires a precocious activation of gut functions. Within the framework of this thesis the intrinsic development of the gut as well as the interaction of the immature gut with its environment were investigated from both a gastroenterologic and a cardiovascular point of view. The obtained insights into developmental biology may enhance our understanding of the complex multifactorial origin of intestinal complications in preterm neonates (Figure 1).

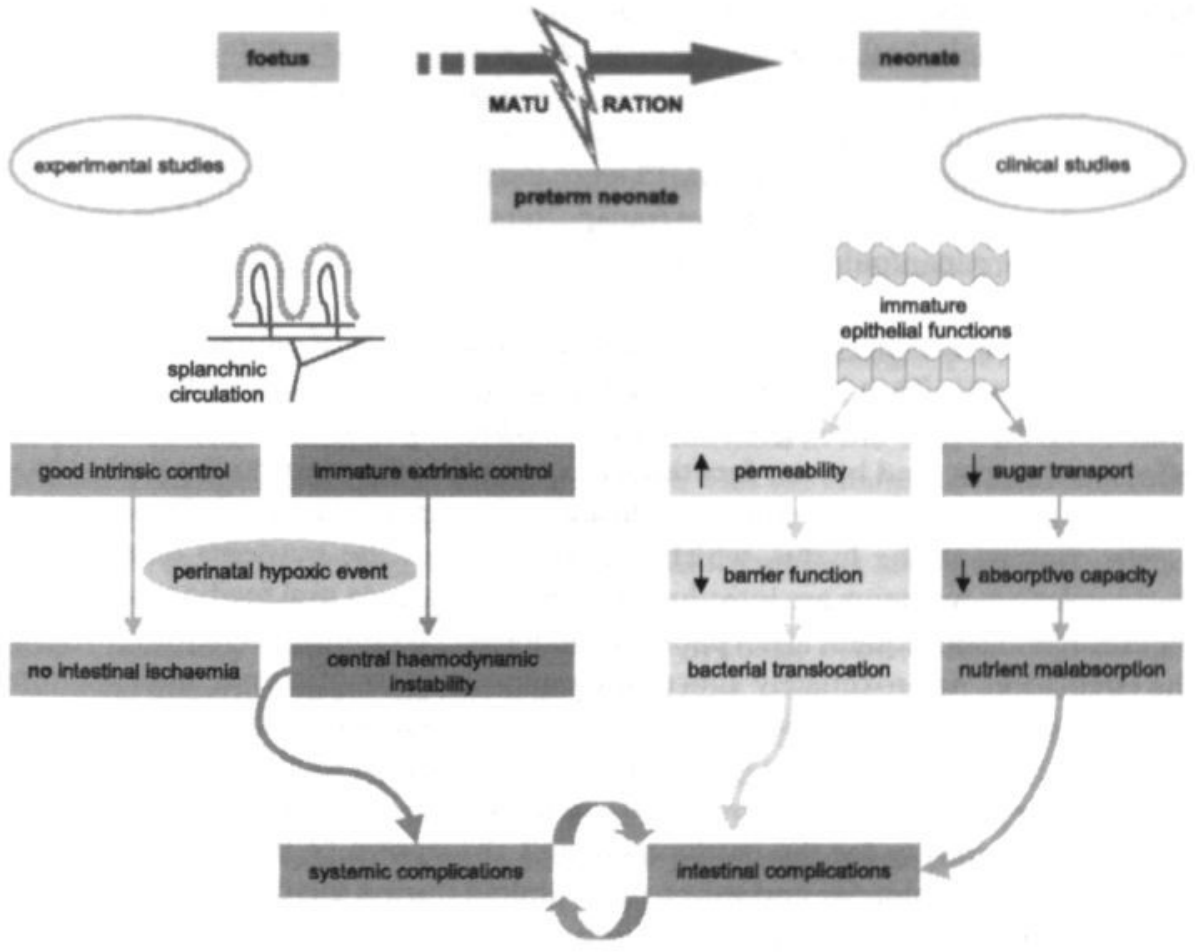

Figure 1

Novel insights into the multifactorial origin of intestinal complications in preterm neonates. 


\section{INTESTINAL MATURATION}

Our observations in preterm neonates provide evidence for the concept that the functional development of the human intestinal tract is partly governed by an internal biologic clock and partly by external triggers (1-5). The appearance of various components of the barrier and nutritive functions of the gut was controlled by an ontogenetic timing mechanism, modulated by dietary factors. Whereas the epithelial barrier function had progressed close to a mature level already by 25 weeks gestation, the integrity of the epithelium deteriorated in the absence of luminal nutrients. Passive and active carrier-mediated monosaccharide absorption started to increase by 30 weeks postconceptional age, independently of the presence of luminal nutrients (Chapter 3). However, the development of the intestinal absorptive capacity for glucose was enhanced by the administration of enteral nutrition shortly after birth (Chapter 4).

This is in line with previous findings in transplantation models. When human small and large intestine were xenografted to mice, the foetal intestine underwent normal morphological differentiation in the absence of exogenous control by luminal factors and systemic hormones (6). Similarly, in rodent models the appearance of sucrase, as well as the induction of fructose absorption and the expression of its apical transporter protein GLUT5 were independent of external signals $(7,8)$. A precocious expression of nutrient transporters such as GLUT5 could be induced by early dietary manipulation (7,9-11).

The time of appearance of a wide range of gut functions is of eminent importance when an infant is born before its intrauterine development has been completed. Premature exposure of the gut to the extrauterine environment in the absence of effective nutritive and barrier functions is a central component to the pathophysiology of intestinal and systemic complications in preterm neonates (Figure 1).

In contrast to the foetus, which receives most of the nutrients directly into the circulation, nutrients are introduced to the neonate via oral feeding that needs digestion and subsequent absorption by the intestinal epithelium before reaching the circulation. Approximately $40 \%$ of the energy content of human milk and preterm infant formula is provided by carbohydrates $(8 \mathrm{~g} / \mathrm{dl})$, mainly in the form of lactose $(12,13)$. The digestion of lactose has been shown to be incomplete prior to 37 weeks gestation due to low lactase activity (14). In addition, we demonstrated that the capacity for the carrier-mediated absorption of the products of the digestive process, glucose and galactose, is limited during the first few weeks after birth in preterm neonates, particularly in neonates born at less than 28 weeks gestation (Chapter 3). Consequently, introduction of oral feeding to a preterm neonate in excess of the digestive and absorptive capacity of the gut may lead to the sequelae of carbohydrate malabsorption.

Fermentation of ingested carbohydrates by bacteria in the lumen of the distal small intestine and colon generates organic acids (e.g., lactate, acetate, butyrate, propionate) and gases (e.g., $\mathrm{H}_{2}, \mathrm{CO}_{2}$, methane) (12,15-17). Although short-chain fatty acids are a major fuel source for colonocytes, excessively high levels of organic acids, especially butyrate, decrease the luminal $\mathrm{pH}$ and cause mucosal damage 
(18). Furthermore, butyrate has been shown to stimulate cytokine release from intestinal epithelial cells (19). In addition, gaseous distension of the intestinal lumen may reduce blood flow in the intestinal wall, resulting in compromised tissue oxygenation, and finally hypoxic injury (20). A role for carbohydrate malabsorption and fermentation in the pathophysiology of NEC is supported by the finding that removal of dietary lactose in a gnotobiotic lactase-deficient animal model in quails reduced the production of organic acids and hydrogen and suppressed NEC-like caecal lesions (21). Furthermore, hydrogen gas has been found in intestinal specimens, and breath hydrogen excretion is increased in patients with NEC $(22,23)$. Low sugar absorptive capacity may thus, as a consequence of carbohydrate malabsorption, predispose the preterm neonate to mucosal injury.

In addition to nutrients, the neonatal gut is exposed to microorganisms. In contrast to the foetus, which develops within the sterile amniotic fluid, the newborn infant must be prepared to deal with bacterial colonisation of the gut and with the ingestion of dietary antigens. To prevent the penetration of potentially harmful substances, the intestinal tract is equipped with an elaborate system of defence mechanisms (24). An important line of defence is constituted by the epithelial monolayer, composed of cells and the intercellular tight junctions (25). We showed that the premature transition from intrauterine to extrauterine life is associated with a temporary reduction in the integrity of the epithelial barrier. This appears to be caused by a disruption of the cellular elements and by modulation of the tight junctions, resulting in an increase in intestinal permeability (Chapter 3 ).

In our study, the intestinal permeability increased during a period of enteral starvation in preterm neonates, and was restored or prevented by enteral nutrition (Chapter 4). This points to a role for enteral nutrition in the origin of these early postnatal changes. Our observations are in line with the changes in intestinal morphology and function that occur in adult patients for whom parenteral nutrition is the sole mode of feeding, including mucosal hypoplasia and increased permeability $(26,27)$. It may be speculated that other factors, including exposure to bacteria, circulatory disturbances and intestinal hypoxia, contribute to the loss of epithelial integrity during the neonatal period (28).

In addition to the physical properties of the epithelial barrier, several key components of the intestinal mucosal immune system are immature in the preterm neonate. These include the germinal centers in Peyer's patches, IgA producing plasma cells in the lamina propria, and intraepithelial lymphocytes, which do not appear until the end of foetal gestation (29). Furthermore, recent studies showed that the level of enteric defensin expression, an antibacterial peptide produced by Paneth cells, is low in the developing gut $(30,31)$. Consequently, the gut of the very low birth weight infant is precociously colonised by an atypical bacterial flora and exposed to dietary antigens in the presence of a vulnerable mucosal barrier.

What may be the consequence of a disrupted intestinal barrier for the preterm neonate? According to the gut origin hypothesis, failure of intestinal barrier function may permit bacterial and endotoxin translocation. This may subsequently trigger splanchnic and systemic cytokine generation and elicit a systemic inflammatory response, leading to sepsis and multiple organ failure (32). Likewise, 
translocation of bacteria and proinflammatory dietary proteins, such as casein, has been suggested in the initiation of intestinal complications and sepsis in preterm neonates $(20,33,34)$. In adult patients increased intestinal permeability, endotoxaemia and/or bacterial translocation have been observed following burn injury, major trauma, sepsis, acute pancreatitis, and major surgery (35-42). Furthermore, bacterial translocation has been associated with an increased incidence of infective complications, at least in immunocompromised patients $(40,43)$. Although these descriptive studies do not provide absolute proof of a causality between intestinal permeability, bacterial translocation, and septic morbidity $(39,44)$, they do support the hypothesis that translocation of bacteria and their products is an important early step in the promotion of sepsis and multiple organ failure in the adult. Our finding of enhanced systemic recovery of bacterial D-lactate in the presence of increased intestinal permeability (Chapter 3 ) is the first indication that disrupted barrier function is accompanied by intrusion of bacterial products from the gut lumen, thereby supporting the gut origin hypothesis in preterm neonates.

It is becoming clear that the intestinal epithelium does not only constitute a physical barrier, but also participates in the mucosal immune response by actively interacting with luminal microorganims through microbial-epithelial crosstalk (45-47). It has been shown that enterocytes are able to mount an innate immune response to bacterial components, such as lipopolysaccharide and peptidoglycan, resulting in the release of inflammatory cytokines. This immune response is mediated by Toll-like receptors present on intestinal epithelial cells (48). In recent investigations these receptors have also been identified on the basolateral surface of human foetal small intestinal crypt enterocytes (49). Moreover, exposure of foetal enterocytes to lipopolysaccharide and interleukin-1 increased the transcription of Toll-like receptors, and generated a greater release of inflammatory cytokines as compared with mature enterocytes (50). Epithelial cytokine production, in turn, can diminish intercellular tight junction integrity and alter intestinal permeability, which further compromises the intestinal barrier (51). It is speculated that exposure of immature intestinal epithelial cells to bacteria leads to an imbalance between release of pro- and anti-inflammatory cytokines from the intestinal mucosa, resulting in an excessive local and systemic inflammatory response $(50,52-54)$. Attenuation of the physical properties and immaturity of the innate immune function of the intestinal epithelium, in conjunction with microbial colonisation of the intestinal tract, may underlie the development of local intestinal and systemic complications in preterm neonates.

From the previous section it is clear that due to low absorptive capacity and vulnerable epithelial barrier function during the early postnatal period (Chapter 3 ), preterm neonates may fail to adapt to the extrauterine environment. Fortunately, the luminal surroundings of the intestinal epithelium are directly amenable to dietary manipulation. This provides the opportunity for the design of clinically applicable interventions to improve the barrier and nutritive functions of the immature gut. Indeed, our studies suggest that enteral nutrients aid in the maintenance of the epithelial integrity, and even induce a precocious enhancement of nutrient transporters (Chapter 4 ). 
These insights into postnatal events that affect gut development contribute to the growing body of data available on gastrointestinal ontogeny, and allow an increasingly rational approach to the nutritional management of very low birth weight infants. Nowadays, protocols for nutritional care vary considerably between neonatal intensive care units due to a scarcity of evidence. It is the fear of feeding related complications by care givers, particularly necrotising enterocolitis, which governs when feedings are started, how rapidly they are advanced, what type of feeding is used, and when they are interrupted. As a consequence, it is still common practice in many neonatal units to provide only total parenteral nutrition initially after birth. The limited absorptive capacity of the intestinal epithelium indeed precludes the administration of large volumes of and rapid advances in enteral feeding. However, complete enteral starvation is accompanied by a deleterious effect on the integrity of the mucosal barrier (Chapter 3). Based on current clinical evidence in preterm neonates, the early introduction of small volumes of human milk or preterm formula shortly after birth supplemented by parenteral feeding to fulfil nutritional and energy requirements is a safe method to support the maturation of intestinal functions which started in utero with the ingestion of amniotic fluid.

In addition to nourishment, enteral feeding may thus also be considered as a form of nutritional therapy to enhance the adaptation of the immature gut to the transition from the intrauterine to the extrauterine environment. Early provision of unmodified human milk or preterm formula is a first step in this process. In recent years, attention in the management of critically ill adult patients has focussed on the use of nutritional support. The optimal route of nutrient delivery in critically ill adults remains controversial $(44,55-60)$. Potentially beneficial effects of supplemented enteral feeding on gut barrier and immune function and reduction in infectious complications have been demonstrated for a wide range of nutrients, including L-glutamine, L-arginine, omega-3 essential fatty acids, and nucleotides (61-63). In preterm neonates enteral glutamine supplementation is safe, and is associated with a reduction in septic events and feeding intolerance (64-66).

Another approach to promote the adaptation of the gut is to manipulate the development and composition of the intestinal microflora in the preterm neonate. One way of influencing the composition of the intestinal microbiota is the oral administration of viable preparations of harmless bacteria, referred to as probiotics. It is purported that the probiotic species occupies the same site as the potential pathogen, and thereby outcompetes the pathogenic strain by colonisation resistance $(67,68)$. Protective effects of probiotic supplementation with bifidobacteria have been demonstrated in animal models of NEC (69-72). Enteral probiotic supplementation with lactobacilli and bifidobacteria may be beneficial in preterm neonates by modulating their colonisation patterns (73-76).

Together, a nutritional program consisting of the early administration of low volumes of enteral nutritional regimens enriched with specific nutrients, such as glutamine and arginine, prebiotics (77), such as fructo-oligosaccharides, and probiotics, such as lactobacilli and bifidobacteria (68,74-76), could be a promising approach to promote the adaptation of the gut, thereby preventing intestinal complications and improving the health status of the neonate during early postnatal life. 
In addition to short-term benefits of nutritional management, nutrition during critical periods of neonatal development may profoundly influence long-term outcomes, a concept known as nutritional programming (78-81). Prospective randomised trials in preterm neonates showed that even a brief period of breast milk consumption is associated with better neurodevelopment, lower blood pressure, and enhanced bone mineralisation during childhood and adolescence (82-85). On the other hand, prolonged breast feeding for more than four months is associated with a reduction in arterial distensibility, as an early marker of cardiovascular disease (86). Likewise, based on our data it may be speculated that dietary manipulation in early life affects the development of gut functioning. This is an additional reason to pay close attention to the nutritional management of preterm neonates. 


\section{VASCULAR MATURATION}

The experimental studies in the chick foetus provide unique insights into the development of the regulation of the perfusion in the immature gut. The balance between intrinsic and extrinsic regulation of intestinal arterial smooth muscle tone shifts from predominant intrinsic control towards extrinsic control. Or, stated otherwise, the level of vasomotor regulation shifts from predominantly local control in young foetuses to central control at the end of foetal development.

Extrapolation of these conclusions to the human situation provides a new concept regarding the role of circulatory disturbances and hypoxia in intestinal complications in preterm neonates. We hypothesise that consequent to the switch from local to central vasomotor control, the origin of intestinal tissue damage in the course of intestinal development shifts from a central to a local problem in oxygen distribution.

Regulation of arterial smooth muscle tone and thereby arterial diameter is a dynamic process, which is determined by the balance between dilator and constrictor mechanisms. We obtained insight into the dynamics of vasomotor control by investigating the mesenteric arterial response to acute hypoxia under physiologic conditions in the intact foetus (Figure 2). Mesenteric arteries between 0.6 and 0.9 of foetal development exhibited a constrictor response during acute hypoxia. The vasoconstriction was rapidly taken over by a dilator response (Chapter 6), and was only maintained in near-term foetuses (Chapter 7). This indicates that the delicate balance of vasomotor regulation shifts from local dilator control to central constrictor control during foetal development.

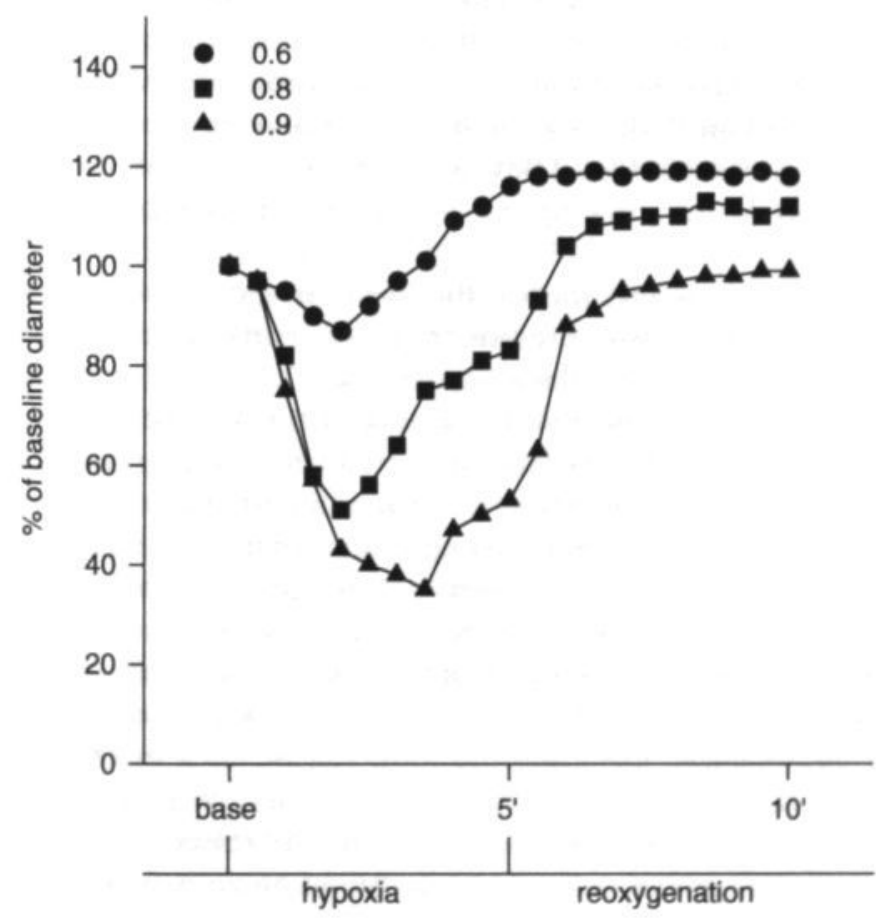

Figure 2

Change in peripheral arterial diameter during acute hypoxia at $0.6-0.9$ of foetal development. 


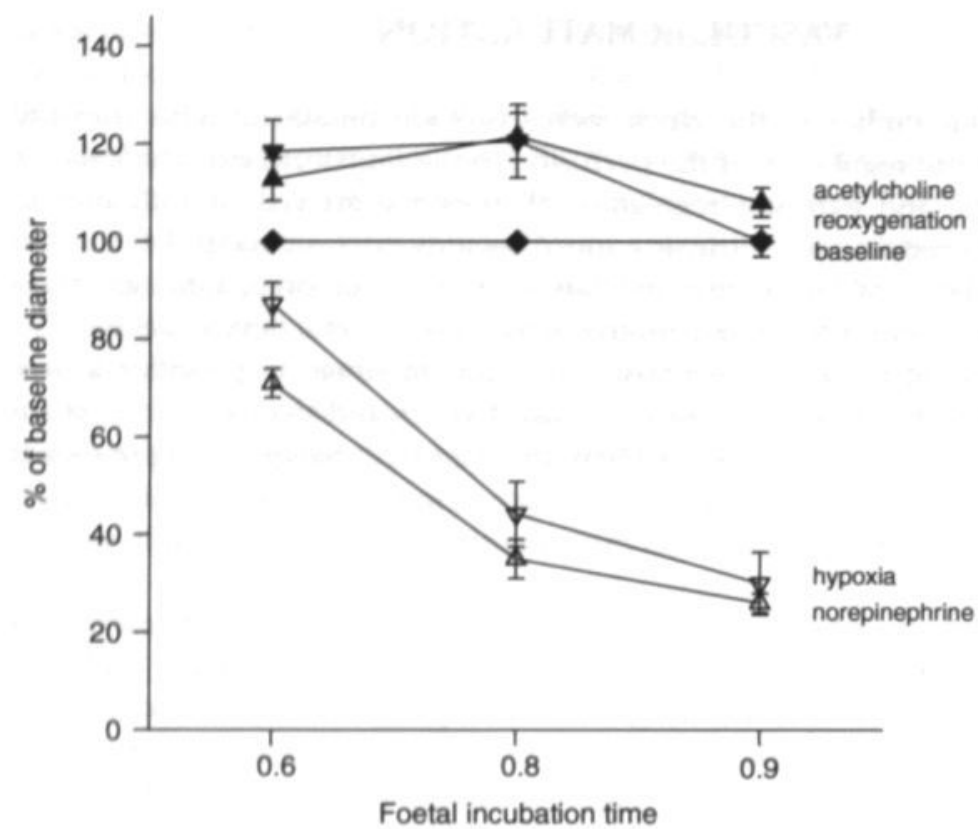

Figure 3

Basal vascular tone in the mesenteric arteries was established by 0.6 of foetal development (Chapter 6). Potential candidates for the establishment of vascular tone identified in the neonatal intestinal circulation include a myogenic response, catecholamines, and endothelin-1 (87). Towards the end of foetal development mesenteric arterial diameter was already maximal under resting conditions (Chapter 7). Thus, in spite of an increase in extrinsic vasoconstrictor control, mesenteric arterial tone under resting conditions decreased towards the end of foetal development (Figure 3). Our findings are in line with previous observations in neonatal piglets, which showed that blood flow is near maximal to meet the relatively high oxygen demand of the growing gut under normal physiologic circumstances (88-90).

Due to the establishment of vascular tone in the foetal mesenteric arteries at 0.6 gestation, the capacity to dilate was present from this early stage onward (Chapter 6). Moreover, the maximal vasodilator capacity of $20 \%$ is similar to that previously reported for the same vessels in adult animals (91). At least some of the vasodilator control in the foetal arteries was conducted via receptor mediated mechanisms, including the adenosine receptor and the muscarinergic receptornitric oxide system (Chapter 6). This confirms the importance of nitric oxide in the local regulation of blood flow in the foetal gut, as it is in the neonatal gut (92-95).

Consequent to the early presence of vasodilator control, the perfusion of the gut was preserved during acute hypoxia despite a serious reduction in blood pressure in the young foetus (Chapter 6). Several potential mechanisms may be responsible for the hypoxia-associated vasodilation, including the local release of a vasodilator metabolite, a direct effect of hypoxia on the vascular wall, and/or a myogenic response. Evidence in support of the latter is provided by the relation between changes in mesenteric arterial diameter and in systemic blood pressure in foetuses 
of different gestational ages (Chapters 6 and 7). During the hypoxic period vasodilation occurred when acute hypoxia was accompanied by a fall in blood pressure, but did not occur when systemic blood pressure remained constant. Furthermore, the increase in arterial diameter generally started during the second minute of hypoxia when arterial pressure reached its lowest level. This indicates that a myogenic response is already established at 0.6 of foetal development, and is an important participant in the intrinsic regulation of the circulation in the developing gut.

Intrinsic vasodilator control of the splanchnic circulation serves to maintain the local perfusion in order to optimise tissue oxygenation necessary for the structural and functional maturation of the developing gut. By the end of foetal development a hierarchical regulation of the circulation is installed, which serves to maintain the oxygen supply to those organs which are essential to the survival of the whole organism, i.e., the heart and brain. To this end, extrinsic neurohumoral constrictor control becomes established during the second half of foetal development in less vital vascular beds, including the splanchnic circulation.

Vasoconstriction mediated by $\alpha$-adrenoceptors was already inducible in the mesenteric resistance arteries by 0.6 gestation, but was not yet involved in the regulation of intestinal arterial tone during hypoxia at this stage (Chapter 6). The adrenergic constrictor capacity of the mesenteric resistance arteries increased considerably between 0.6 and 0.9 of foetal development (Chapter 7). The most important mediators of the humoral control of the foetal mesenteric resistance arteries are $\alpha$-adrenoceptors, as indicated by the finding that the hypoxia-associated constriction was prevented for $95 \%$ by the $\alpha$-adrenoceptor antagonist phentolamine (Chapter 6). This implicates that the proposed contribution of other constrictor agents, such as endothelin, vasopressin, and angiotensin II (96-100), as well as an effect of hypoxia itself on vascular smooth muscle $(101,102)$, is negligible in vivo. As a consequence of enhanced adrenergic constrictor capacity, the magnitude of the constrictor response to acute hypoxia increased during the second half of foetal gestation (Chapter 6).

Our findings also shed light on the role of the sympathetic nervous system in the vascular response to hypoxia in the foetus. Functional neural control of the intestinal circulation by perivascular sympathetic nerves was only attained at 0.9 of foetal gestation (Chapter 7). Considering this fact, sympathetic neural control is not involved in the $\alpha$-adrenoceptor mediated constrictor response to hypoxia prior to this stage. It is therefore highly likely that after the appearance of functional $\alpha$-adrenergic receptors on the vascular smooth muscle cells, adrenergic control is initially exerted by circulating catecholamines released from the adrenal gland as a direct consequence of a reduction in blood oxygen concentration (103-105).

This sequence of the maturation process has obvious consequences for the regulation of the perfusion of the developing gut. Due to the relatively limited $\alpha$-adrenergic pharmacomechanical coupling in the presence of already well established intrinsic vasodilator properties, intestinal blood flow during acute hypoxia was preserved prior to 0.9 of foetal development (Chapter 6). Vasoconstriction started to predominate following the establishment of neural control of the intestinal circulation, leading to a fall in gut perfusion during acute hypoxia (Chapter 7). 


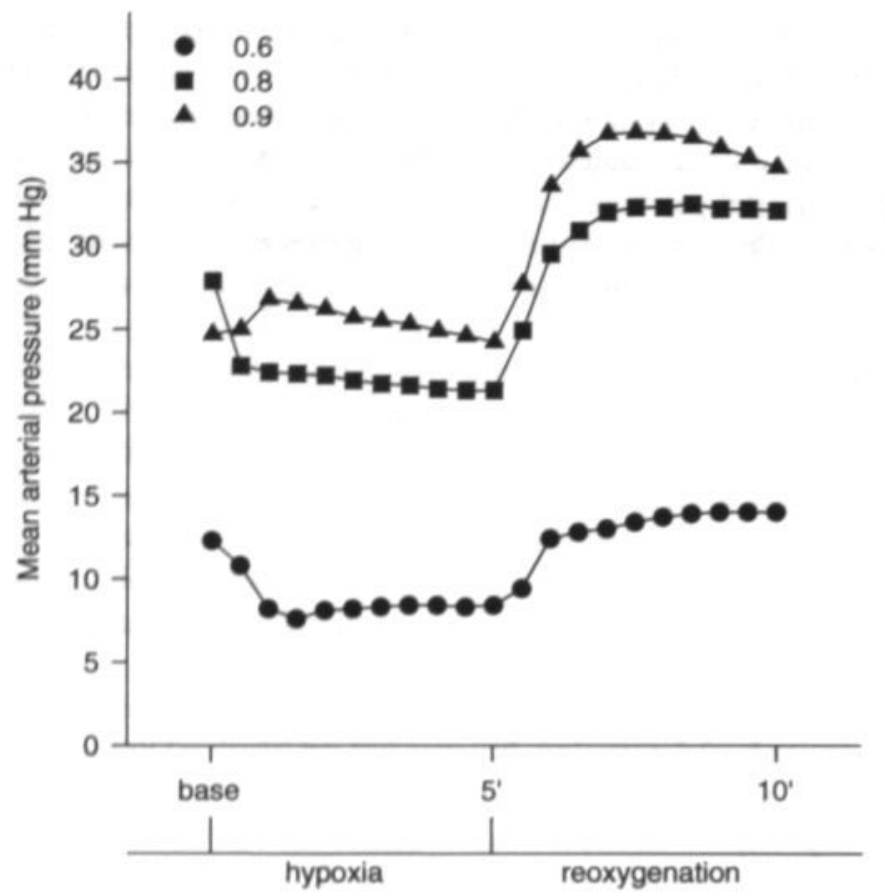

Figure 4

Change in arterial pressure during acute hypoxia at $0.6-0.9$ of foetal development.

Even more important are the implications of local vasomotor control in individual vascular beds for the integrated regulation of systemic haemodynamics (Figure 4). Acute hypoxia was accompanied by a strong decrease in heart rate in all foetuses between 0.6 and 0.9 of development (Chapter 7). Since heart rate is a major determinant of the cardiac output, bradycardia is accompanied by a drop in cardiac output. The latter, in turn, leads to a fall in blood pressure, unless it is compensated by a simultaneous rise in peripheral vascular resistance. Assuming that the splanchnic circulation is representative of other major peripheral vascular beds, including the renal tract and musculoskeletal system (106), predominance of local vasodilator control prior to 0.9 of foetal development precluded a compensatory increase in peripheral vascular resistance, resulting in a decrease in arterial pressure during acute hypoxia (Chapter 6). Dominance of vasoconstriction in the peripheral vasculature in foetuses near term resulted in maintenance of the systemic blood pressure during acute hypoxia (Chapter 7), which is vital to preserve the perfusion and oxygenation of the heart and brain in order to protect the survival of the whole organism (107). In conclusion, the maturation process of vasomotor control has important consequences for the distribution of oxygen when oxygen supply to the developing organism becomes compromised. Consequent to the shift from local to central control of vasomotor tone, the level of oxygen distribution shifts at the expense of the individual organs in favour of the survival of the organism. 
Extrapolation of these conclusions provides a new concept regarding the role of circulatory disturbances and hypoxia in intestinal complications in preterm neonates (Figure 1). In previous reports it was postulated that the intestine becomes ischaemic during a perinatal hypoxic event, due to exaggerated vasoconstriction and/or insufficient autoregulation of the intestinal circulation. Subsequent restoration of the oxygen supply to the gut was supposed to induce an inflammatory response, causing further damage to the gut tissue, ultimately resulting in necrotising enterocolitis (Chapter 2). However, these reports did not take into account the aspect of development, i.e., that the preterm neonate is born in the course of the second half of foetal gestation. Our experimental data suggest that the immature gut is actually well protected from ischaemia-reperfusion injury. First, perfusion of the developing gut was preserved during hypoxic periods, due to the early establishment of local vasodilator control (Chapter 6). Second, the developing gut was protected from reoxygenation damage following episodes of hypoxia, due to immaturity of leukocyte-vessel wall interactions, as part of a local inflammatory response (Chapter 5). In view of the switch from local to central vasomotor control with increasing gestational age, a role for local gut ischaemia in the initiation of tissue damage, if any, is only likely in (near) term neonates.

These findings are consistent with the epidemiologic evidence. Perinatal hypoxic events are more common in near- and full-term neonates afflicted with NEC as compared with healthy gestational age-matched controls. Moreover, in these neonates NEC usually develops within several days after such an event (108111 ). In preterm neonates, however, such a consistent causal or temporal association between perinatal hypoxic events and NEC has not been demonstrated (112115).

Temporary reductions in arterial oxygen content due to respiratory and cardiac distress are common in preterm neonates (116). As we showed, the ability to maintain cardiovascular homeostasis under conditions of compromised oxygen supply depends on the level vasomotor control. We hypothesise that premature exposure to the extrauterine environment, especially at less than 32 weeks (less than 0.8 gestation), vasomotor control may still be inadequate to anticipate episodes of hypoxia. A generalised failure of systemic haemodynamic control may contribute to intestinal complications in preterm neonates (Figure 1). 


\section{CONCLUDING REMARKS}

The number of preterm births, especially very low birth weight infants, has steadily risen during the last two decades. This appears largely attributable to the increased use of earlier obstetric interventions for pathologic pregnancies, such as those complicated by severe pre-eclampsia, foetal growth retardation, and prolonged rupture of membranes, the increase in the rate of multiple births (caused largely by treatment for infertility), and the increase in the proportion of deliveries to women aged 35 years or older (117-119). In addition, specific perinatal and neonatal therapeutic interventions, such as antenatal steroids and exogenous surfactants, have contributed to continued improvement in the survival of very low birth weight neonates (120-122).

Giving premature birth to an infant is traumatic for parents, and the provision of intensive care to these patients is expensive. Preterm birth is the most important child health problem in developed societies, as it is the leading cause of infant mortality and is associated with major short-term as well as long-term respiratory, intestinal, ophthalmologic, and neurodevelopmental morbidity $(123,124)$. There is a growing appreciation that external triggers, including nutrition and oxygenation status, during critical windows of development may profoundly influence long. term outcomes. This adds a new dimension to the care of the preterm neonate and provides a potential opportunity to prevent such long-term sequelae. The growing number of very low birth weight neonates and their specific pathologies underlines the need for further basic experimental and clinical research in advancing our understanding of the maturation processes involved in the adaptation to extrauterine life. 


\section{References}

1. Sanderson I.R. The physicochemical environment of the neonatal intestine. Am J Clin Nutr 1999; 69 (suppl): 1028S-1034S.

2. Sanderson L.R. Dietary regulation of genes expressed in the developing intestinal epithelium. Am J Clin Nutr 1998; 68: 9991005.

3. Montgomery R.K., Mulberg A.E, and Grand R.J. Development of the human gastrointestinal tract: twenty years of progress. Gastroenterology 1999, 116: 702-731.

4. Thiesen A., Wild G., Keelan M., Clandinin M.T., McBurney M., Van Aerde J., and Thomson A.B.R. Ontogeny of intestinal nutrient transport. Can J Physiol Pharmacol 2000; 78: 513-527.

5. Pacha J. Development of intestinal transport function in mammals. Physiol Rev 2000; 80: 1633-1667.

6. Savidge T.C., Morey A.L., Ferguson D.J., Fleming K.A., Shmakov A.N., and Phillips A.D. Human intestinal development in a severe-combined immunodeficient xenograft model. Differentiation 1995; 58 : 361-371.

7. Shu R., David E.S., and Ferraris R.P. Dietary fructose enhances intestinal fructose transport and GLTS expression in weaning rats. Am J Physiol 1997; 272: G446-G453.

8. Redel C.A., Shulman R.J., and Tivey D.R. Determinants of lactose digestion in the miniature pig. Dig Dis Sci 1997; 42: 137-144.

9. Shu R., David E.S., and Ferraris R.P. Luminal fructose modulates fructose transport and GLUT-5 expression in small intestine of weaning rats. Am J Physiol 1998; 274: G232-G239.

10. Monteiro I.M., Jiang L., and Ferraris R.P. Dietary modulation of intestinal fructose transport and GLTS mRNA expression in hypothyroid rat pups. J Pediatr Gastroenterol Nutr 1999, 29: 563-570.

11. Trahair J.F. and Sangild P.T. Systemic and luminal influences on the perinatal development of the gut. Equine Vet J 1997; 24: 40-50.

12. Kien C.L. Digestion, absorption, and fermentation of carbohydrates in the newborn. Clin Perinatol 1996; 23: 211-228.

13. Kunz C., Rodriguez-Palmero M., Koletzko B., and Jensen R. Nutritional and biochemical properties of human milk (part I): General aspects, proteins, and carbohydrates. Clin Perinatol 1999; 26: 307-333.

14. Shulman R.J., Feste A., and Ou C. Absorption of lactose, glucose polymers, or combination in premature infants. J Pediatr 1995; 127: 626-631.

15. Kien C.L. Colonic fermentation of carbohydrate in the premature infant: possible relevance to necrotizing enterocolitis. J Pediatr 1990; 117: S52-S57.

16. Olesen M., Gudmand-Hoyer E., Holst J.J., and Jorgensen S. Importance of colonic bacterial fermentation in short bowel patients; small intestinal malabsorption of easily digestible carbohydrate. Dig Dis Sci 1999; 44: 1914-1923.

17. Macfarlane G.T. and Macfarlane S. Human colonic microbiota: ecology, physiology and metabolic potential of intestinal bacteria. Scand J Gastroenterol Suppl 1997; 222: 3-9.

18. Murray R.D. Effects of bacterial fermentation end products on intestinal function: implications for intestinal dysfunction.J Pediatr 1990; 117: S59-S63.

19. Fusunyan R.D., Quinn J.J., and Ohno Y. Butyrate enhances IL-8 secretion from intestinal epithelial cells in response to IL-1 beta and lipopolysaccharide. Pediatr Res 1998; 43: 8490.

20. Clark D.A. and Miller M.J.S. Intraluminal pathogenesis of necrotizing enterocolitis. J Pediatr 1990; 117 : S64.S67.

21. Szylit 0., Butel M.J., and Rimbault A. An experimental model of necrotising enterocolitis. Lancet 1997; 350: 33-34. 
22. Cheu H.W., Brown D.R., and Rowe M.I. Breath hydrogen excretion as a screening test for the early diagnosis of necrotizing enterocolitis. Am J Dis Child 1989, 143: 156-159.

23. Engel R.R., Virning N.L., Hunt C.E., and Levitt M.D. Origin of mural gas in necrotizing enterocolitis. Pediatr Res 1973; 7: 292.

24. Sanderson L.R. and Walker W.A. Mucosal Barrier: an Overview. In: Ogra P.L., Mestecky J., Lamm M.E., Strober W., Bienenstock J., and MoGhee J.R., editors. Mucosal Immunology. 2nd ed. San Diego:Academic Press, 1999: 5-17.

25. Nusrat A., Turner J.R., and Madara J.L. Molecular physiology and pathophysiology of tight junctions. IV. Regulation of tight junctions by extracellular stimuli: nutrients, cytokines, and immune cells. $\mathrm{Am} \mathrm{J}$ Physiol 2000; 279: G851-6857.

26. Maxton D.G., Menzies L.S., Slavin B., and Thompson R.P.H. Small-intestinal function during enteral feeding and starvation in man. Clin Sci 1989, 77: 401-406.

27. Buchman A.L., Moukarzel A.A., Bhuta S., Belle M., Ament M.E., Eckhert C.D., Hollander D., Gornbein J., Kopple J.D., and Vijayaroghavan S.R. Parenteral nutrition is associated with intestinal morphologic and functional changes in humans.J Parenter Enter Nutr 1995; 19: 453-460.

28. Xu D.-Z., Lu Q., Kubicka R., and Deitch E.A. The effect of hypoxia/reoxygenation on the cellular function of intestinal epithelial cells.J Trauma 1999; 46: 280-285.

29. Insoft R.M., Sanderson I.R., and Walker W.A. Development of immune function in the intestine and its role in neonatal diseases. Pediatr Clin North Am 1996; 43: 551-571.

30. Mallow E.B., Harris A., Salzman N., Russell J.P., DeBerardinis R.J., Ruchelli E., and Bevins C.L. Human enteric defensins; gene structure and developmental expression.J Biol Chem 1996; 271: 4038-4045.

31. Salzman N., Polin R.A., Harris M.C., Ruchelli E., Hebra A., Zirin-Butler S., Jawad A., Porter E.A., and Bevins C.L. Enteric defensin expression in necrotizing enterocolitis. Pediatr Res 1998; 44: 20-26.

32. Swank G.M. and Deitch E.A. Role of the gut in multiple organ failure: bacterial translocation and permeability changes. World J Sung 1996; 20: 411-417.

33. Deitch E.A. Role of bacterial translocation in necrotizing enterocolitis. Acta Paediatr Suppl 1994; 396: 33-36.

34. Clark D.A., Thompson J.E., Weiner L.B., McMillan J.A., Schneider A.J., and Rokahr J.E. Necrotizing enterocolitis: intraluminal biochemistry in human neonates and a rabbit model. Pediatr Res 1985; 19 : 919921.

35. LeVoyer T., Cioffi W.G.J., and Pratt L. Alterations in intestinal permeability after thermal injury. Arch Sung 1992; 127: 26-29.

36. Pape H.C., Dwenger A., and Regel G. Increased gut permeability after multiple trauma. Br J Surg 1994; 81: $850-852$.

37. Johnston J.D., Harvey C.J., Menzies I.S., and Treacher D.F. Gastrointestinal permeability and absorptive capacity in sepsis. Crit Care Med 1996; 24: 1144-1149.

38. Juvonen P.O., Alhava E.M., and Takala J.A. Gut permeability in patients with acute pancreatitis. Scand J Gastroenterol 2000; 12: 1314-1318.

39. Kanwar S., Windsor A.C., Welsh F., Barclay G.R., Guillou P.J., and Reynolds J.V. Lack of correlation between failure of gut barrier function and septic complications after major upper gastrointestinal surgery. Ann Sung 2000; 231: 88-95.

40. O'Boyle C.J., MacFie J., Mitchell C.J., Johnston J.D., Sagar P.M., and Sedman P.C. Microbiology of bacterial translocation in humans. Gut 1998; 42: 2935.

41. Riddington D.W., Venkatesh B., Boivin C.M., Bonser R.S., Elliott T.S.J., Marshall T., Mountford P.J., and Bion J.F. Intestinal permeability, gastric intramucosal $\mathrm{pH}$, and systemic endotoxemia in patients undergoing cardiopulmonary bypass. JAMA 1996; 275: 1007-1012. 
42. Winchurch RA., Thupari J.N., and Munster A.M. Endotoxemia in burn patients: levels of circulating endotoxins are related to burn size. Surgery 1987; 102:808-812.

43. MacFie J., O'Boyle C., Mitchell C.J., Buckley P.M., Johnstone D., and Sudworth P. Gut origin of sepsis: a prospective study investigating associations between bacterial translocation, gastric microflora, and septic morbidity. Gut 1999; 45: 223-228.

44. MacFie J. Enteral versus parenteral nutrition: the significance of bacterial translocation and gut-barrier function. Nutrition 2000, 16:606-611.

45. Bloom P.D. and Boedecker E.C. Mucosal immune responses to intestinal bacterial pathogens. Semin Gastrointest Dis 1996; 7: 151-166.

46. Medzhitov R. and Janeway C. Innate immunity. N Engl J Med 2000; 343:338-344.

47. MoCormick B.A., Gerwirtz A.T., and Madara J.L. Epithelial cross-talk with bacteria and immune cells. Curr Opin Gastmenterol 1998; 14: 492-497.

48. Aderen A. and Ulevitch R.J. Toll-like receptors in the induction of the innate immune response. Natum 2000; 406: 782-787.

49. Fusunyan R.D., Nanthakumar N.N., Baldeon M.E., and Walker W.A. Evidence for an innate immune response in the immature human intestine: toll-like receptors on fetal enterocytes. Pediatr Res 2001; 49 : 589593.

50. Nanthakumar N.N., Fusunyan R.D., Sanderson L., and Walker W.A. Inflammation in the developing human intestine: a possible pathophysiologic contribution to necrotizing enterocolitis. Proc Natl Acad Sci USA 2000; 97: 6043-6048.

51. McKay D.M. and Baird A.W. Cytokine regulation of epithelial permeability and ion transport. Gut 1999; 44: 283-289.

52. Shah U. and Walker W.A. Adverse host responses to bacterial toxins in human infants. J Nutr 2000; 130: 420S-425S.

53. Nadler E.P., Dickinson E., Knisely A., Zhang X.R., Boyle P., Beer-Stolz D., Watkins S.C., and Ford H.R. Expression of inducible nitric oxide synthase and interleukin-12 in experimental necrotizing enterocolitis. J Sung Res 2000; 92: 71-77.

54. Edelson M.B., Bagwell C.E., and Rozycki H.J. Circulating pro- and counterinflammatory cytokine levels and severity in necrotizing enterocolitis. Pediatrics 1999; 103: 766-771.

55. Reynolds J.V., Kanwar S., Welsh F.K.S., Windsor A.C.J., Murchan P., Barclay G.R., and Guillou P.J. Does the route of feeding modify gut barrier function and clinical outcome in patients after major upper gastrointestinal surgery? J Parenter Enter Nutr 1997; 21: 196-201.

56. Braga M., Gianotti L., Gentilini O., Parisi V., Salis C., and Di Carlo V. Early postoperative enteral nutrition improves gut oxygenation and reduces costs compared with total parenteral nutrition. Crit Care Med 2001; 29: 242-248.

57. Woodcock N.P., Zeigler D., Palmer M.D., Buckley P., Mitchell C.J., and MacFie J. Enteral versus parenteral nutrition: a pragmatic study. Nutrition 2001; 17: 1-2.

58. Moore F.A., Feliciano D.V., Andrassy R.J., McArdle A.H., Booth F.V., Morganstein-Wagner T.B., Kellum J.M., Welling R.E., and Moore E.E. Early enteral feeding, compared with parenteral reduces postoperative septic complications - the results of a meta-analysis. Ann Sung 1992; 216: 172-183.

59. Minard G. and Kudsk K.A. Nutritional support and infection: does the route matter? World J Surg 1998; 22: 213-219.

60. Moore F.A. Common mucosal immunity: a novel hypothesis. Ann Sung 2000; 231:9-10.

61. Heys S.D., Walker L.G., Smith I., and Eremin 0. Enteral nutritional supplementation with key nutrients in patients with critical illness and cancer. Ann Surg 1999, 229: 467-477. 
62. Rowlands B.J. and Gardiner K.R. Nutritional modulation of gut inflammation. Proc Nutr Soc 1998; 57: 395-401.

63. Bower R.H., Cerra F.B., Bershadsky B., Licari J.J., Hoyt D.B., Jensen G.L., Van Buren C.T., Rothkopf M.M., Daly J.M., and Adelsberg B.R. Early enteral administration of a formula (Impact) supplemented with arginine, nucleotides, and fish oil in intensive care unit patients: results of a multicenter, prospective, randomized, clinical trial. Crit Care Med 1995; 23: 436-449.

64. Dallas M.J., Bowling D., Roig J.C., Auestad N., and Neu J. Enteral glutamine supplementation for very. low-birth-weight infants decreases hospital costs. J Parenter Enter Nutr 1998; 22: 352-356.

65. Neu J., DeMarco V., and Weiss M. Glutamine supplementation in low-birth-weight infants: mechanisms of action. J Parenter Enter Nutr 1999; 23: S49S51.

66. Neu J., Roig J.C., and Meetze W.H. Enteral glutamine supplementation for very low birth weight infants decreases morbidity. J Pediatr 1997; 131: 691-699.

67. Mackie R.I., Sghir A., and Gaskins H.R. Developmental microbial ecology of the neonatal gastrointestinal tract. Am J Clin Nutr 1999; 69 (suppl): 1035S-1045S.

68. Duffy L.C. Interactions mediating bacterial translocation in the immature intestine. J Nutr 2000; 130: 432S-436S.

69. Caplan M.S., Miller-Catchpole R., Kaup S., Russell T., Lickerman M., Amer M., Xiao Y., and Thomson R. Bifidobacterial supplementation reduces the incidence of necrotizing enterocolitis in a neonatal rat model. Gastroenterology 1999; 117: 577-583.

70. Catala L., Butel M.J., Bensaada M., Popot F., Tessedre A.C., Rimbault A., and Szylit 0. Oligofructose contributes to the protective role of bifidobacteria in experimental necrotising enterocolitis in quails.

JMed Microbiol 1999; 48: 8994.

71. Butel M.J., Roland N., Hibert A., Popot F., Favre A., Tessedre A.C., Bensaada M., Rimbault A., and Szylit 0. Clostridial pathogenicity in experimental necrotising enterocolitis in gnotobiotic quails and protective role of bifidobacteria. JMed Microbiol 1998; 47: 391-399.

72. Butel M.J. and Szylit 0. Bifidobacteria in necrotizing enterocolitis. Gastroenterology 2000; 118: 1280-1281.

73. Caplan M.S. and Jilling T. Neonatal necrotizing enterocolitis: possible role of probiotic supplementation. J Pediatr Gastmoenterol Nutr 2000; 30: S18-S22.

74. Millar M.R., Bacon C., Smith S.L., Walker V., and Hall M.A. Enteral feeding of premature infants with Lactobacillus GG. Anch Dis Child 1993; 69: 483-487.

75. Kitajima H., Sumida Y., Tanaka R., Yuki N., Takayama H., and Fujimura M. Early administration of Bifidobacterium breve to preterm infants: randomised controlled trial. Anch Dis Child 1997; 76: F101-F107.

76. Gronlund M.M., Lehtonen O.P., Kero P., Saxelin M., and Salminen S. Lactobacillus GG supplementation does not reduce faecal colonization of Klebsiella axytoca in preterm infants. Acta Paediatr 1997; 86: 785-786.

77. Gibson G.R. and Roberfroid M.B. Dietary modulation of the human colonic microflora: introducing the concept of prebiotics. J Nutr 1995; 125: 1401-1412.

78. Lucas A., Morley R., and Cole T.J. Randomised trial of early diet in preterm babies and later intelligence quotient. BMJ 1998; 317: 1481-1487.

79. Morley R. and Lucas A. Influence of early diet on outcome in preterm infants. Acta Paediatr Suppl 1994; 405: $123-126$.

80. Lucas A. Programming by early nutrition: an experimental approach. J Nutr 1998; 128: 401S-406S.

81. Lucas A. Role of nutritional programming in determining adult morbidity. Arch Dis Child 1994; 71: 288-290. 
82. Lucas A, Morley R., Cole T.., Lister G., and Lesson-Payne C. Breast milk and subsequent intelligence quotient in children born preterm. Lancet 1992; 339. 261-264.

83. Singhal A., Cole T.J., and Lucas A. Early nutrition in preterm infants and later blood pressure: two cohorts after randomised trials. Lancet 2001; 357: 413-419.

84. Bishop N.J., Dahlenburg S.L., Fewtrell M.S., Morley R, and Lucas A. Early diet of preterm infants and bone mineralisation at age 5 years. Acta Paediatr Scand 1996; 85: 230-236.

85. Lucas A., Morley R., Cole T.J, and Gore S.M. A randomised multicenter study of human milk versus formula and later development in preterm infants. Anch Dis Child 1994; 70: F141-F146.

86. Leeson C.P., Kattenhorn M., Deanfield J.E., and Lucas A. Duration of breast feeding and arterial distensibility in early adult life: population based study. BMJ 2001; 322: 643-647.

87. Nankervis CA., Dunaway D.J., and Nowicki P.T. Determinants of terminal mesenteric artery resistance during the first postnatal month. Am J Physiol 2001; 280: G678-G686.

88. Nowicki P.T. Neonatal intestinal circulation. In: Handbook of Physiology. Bethesda: American Physiological Society, 1989: 1597-1603.

89. Nowicki P.T., Miller C.E., and Edwards R.C. Effects of hypoxia and ischemia on autoregulation in postnatal intestine. Am J Physiol 1991; 261: G152-G157.

90. Nowicki P.T. and Miller C.E. Effect of increased tissue oxygen uptake on autoregulation in postnatal intestine. Am J Physiol 1992; 263: G690-G694.

91. Mian R. and Marshall J.M. The role of adenosine in mediating vasodilatation in mesenteric circulation of the rat in acute and chronic hypoxia. J Physiol 1995; 489: 225-234.

92. Nowicki P.T. and Nankervis C.A. The role of the circulation in the pathogenesis of necrotizing enterocolitis. Clin Perinatol 1994; 21: 219233.

93. Nowicki P.T. The effects of ischemia-reperfusion on endothelial cell function in postnatal intestine. Pediatr Res 1996; 393: 267-274.

94. Nowicki P.T. Postnatal changes in gut hemodynamics: a possible role for substance P.Am J Physiol 1998; 274: G1142-G1150.

95. Reber K.M., Mager G.M., Miller C.E., and Nowicki P.T. Relationship between flow rate and NO production in postnatal mesenteric arteries. Am J Physiol 2001; 280: G43-G50.

96. Green L.R., McGarrigle H.H.G., Bennet L., and Hanson M.A. Angiotensin II and cardiovascular chemoreflex responses to acute hypoxia in late gestation fetal sheep.J Physiol 1998; 507: 857-867.

97. Wadsworth R.M. Vasoconstrictor and vasodilator effects of hypoxia. TPSS 1994; 15: 47-53.

98. Raff H. and Wood C.E. Effect of age and blood pressure on the heart rate, vasopressin, and renin response to hypoxia in fetal sheep. Am J Physiol 1992; 263: R880-R884.

99. Perez R., Espinoza M., Riquelme R., Parer J.T., and Llanos A.J. Arginine vasopressin mediates cardiovascular responses to hypoxemia in fetal sheep. Am J Physiol 1989; 256: R1011-R1018.

100. Rose C.E., Godine R.L., Rose K.Y., Anderson R.J., and Carey R.M. Role of arginine vasopressin and angiotensin II in cardiovascular responses to combined acute hypoxemia and hypercapnic acidosis in conscious dogs. J Clin Invest 1984; 74: 321-331.

101. Aalkjaer C. and Lombard J.H. Effect of hypoxia on force, intracellular $\mathrm{pH}$ and $\mathrm{Ca}^{2+}$ concentration in rat cerebral and mesenteric small arteries. J Physiol 1995; 482: 409419.

102. Nankervis C.A. and Miller C.E. Developmental differences in response of mesenteric artery to acute hypoxia in vitro. Am J Physiol 1998; 274: G694-G699.

103. Thompson R.J., Jackson A., and Nurse C.A. Developmental loss of hypoxic chemosensitivity in rat adrenomedullary chromaffin cells. J Physiol 1997; 489: 503-510.

104. Rychkov G.Y., Adams M.B., McMillen I.C., and Roberts M.L. Oxygen-sensing mechanisms are present in the chromaffin cells of the sheep adrenal medulla before birth. J Physiol 1998; 509: 887-893. 
105. Inoue M., Fujishiro N., and Imanaga L. Hypoxia and cyanide induce depolarization and catecholamine release in dispersed guinea-pig chromaffin cells. J Physiol 1998; 507: 807-818.

106. Mulder A.L.M., Van Golde J.C., Prinzen F.W., and Blanco C.E. Cardiac output distribution in response to hypoxia in the chick embryo in the second half of the incubation time. J Physiol 1998; 508: 281-287.

107. Jensen A., Hohmann M., and Kuenzel W. Dynamic changes in organ blood flow and oxygen consumption during acute asphyxia in fetal sheep. J Dev Physiol 1987; 9: 543-559.

108. Andrews D.A., Sawin R.S., Ledbetter D.J., Schaller R.T., and Hatch E.I. Necrotizing enterocolitis in term neonates. Am J Surg 1990; 159: 507-509.

109. Wilson R., Kanto W.P., McCarthy B.J., Burton A., Lewin P., and Feldman R.A. Age at onset of necrotizing enterocolitis: an epidemiologic analysis. Pediatr Res 1982; 16: 82-84.

110. Wiswell T.E., Robertson C.F., Jones T.A., and Tuttle D.J. Necrotizing enterocolitis in full-term neonates, a case-control study. Am J Dis Child 1988; 142: 532-535.

111. De Gamarra E., Helardot P., Moriette G., Murat I., and Relier J.P. Necrotizing enterocolitis in full-term newborns. Biol Neonate 1983; 44: 185-192.

112. Beeby P.J. and Jeffery H. Risk factors for necrotizing enterocolitis: the influence of gestational age. Anch Dis Child 1992; 67: 432-434.

113. Kanto W.P., Wilson R., Breart G.L., Zierler S., Purohit D.M., Peckham G.J., and Ellison C. Perinatal events and necrotizing enterocolitis in premature infants. Am J Dis Child 1987; 141: 167-169.

114. Stoll B.J. Epidemiology of necrotizing enterocolitis. Clin Perinatol 1994; 21: 205-218.

115. Kliegman R.M., Hack M., Jones P., and Fanaroff A.A. Epidemiologic study of necrotizing enterocolitis among low-birth-weight infants; absence of identifiable risk factors. J Pediatr 1982; 100: 440-444.

116. Adams J.A., Zabaleta I.A., and Sackner M.A. Hypoxemic events in spontaneously breathing premature infants: etiologic basis. Pediatr Res 1997; 42: 463-471.

117. Kramer M.S., Platt R., Yang H., Joseph K.S., Wen L.W., Morin L., and Usher R.H. Secular trends in preterm birth; a hospital-based cohort study. JAMA 1998; 280: 1849-1854.

118. Joseph K.S., Kramer M.S., and Marcoux S. Determinants of secular trends in preterm birth in Canada. NEngl J Med 1998; 339: 14341439.

119. Den Ouden A.L. Toename van het aantal vroeggeboorten in Nederland: vergelijking van 1983 en 1993. Ned Tijdschr Geneeskd 1998; 142: 127-131.

120. Kliegman R.M. Neonatal technology, perinatal survival, social consequences, and the perinatal paradox. Am J Public Health 1995; 85: 909913.

121. Hack M., Horbar J.D., and Malloy M.H. Very low birth weight outcomes of the NICHD neonatal network. Pediatrics 1991; 87: 587-597.

122. Hack M., Friedman H., and Fanaroff A.A. Outcomes of extremely low-birth weight infants. Pediatrics 1996; 98: 931-937.

123. Vohr B.R., Wright L.L., Dusick A.M., Mele L., Verter J., Steichen J.J., Simon N.P., Wilson D.C., Broyles S., Bauer C.R., Delaney-Black V., Yolton K.A., Fleisher B.E., Papile L.A., and Kaplan M.D. Neurodevelopmental and functional outcomes of extremely low birth weight infants in the National Institute of Child Health and Human Development Neonatal Research Network, 1993-1994. Pediatrics 2000; 105: 1216-1226.

124. Dollfus C., Paletta M., Siegel E., and Cross A.W. Infant mortality. Pediatrics 1990; 86: 176-183. 
Intestinal complications such as necrotising enterocolitis are at present main causes of morbidity and mortality among preterm neonates, in particular very low birth weight neonates. This topic is introduced in Chapter 1. Several factors are implied in the pathogenesis of intestinal complications, including enteral feeding, intestinal bacterial flora, and disturbances in intestinal perfusion and oxygenation. However, the lack of a comprehensive understanding of the underlying pathophysiologic mechanisms has precluded the development of effective preventive measures. Since the risk of intestinal complications is related to the degree of prematurity, it appears that intestinal complications are the final outcome of the interaction between an immature gastrointestinal tract and potentially injurious exogenous influences. The preterm neonate is exposed to the stresses of extrauterine life ahead of time, requiring precocious adaptation of its intestinal tract and cardiovascular system. It is hypothesised that a failure to adapt to the premature transition from the intrauterine to the extrauterine environment predisposes the preterm neonate to intestinal complications.

In the first part of Chapter $\mathbf{2}$ an overview is given of the current knowledge regarding the development of the human gut. Attention is addressed to the intestinal nutritive and barrier functions, i.e., the ability to absorb nutrients from ingested food, and to prevent invasion of potentially harmful luminal factors. In addition, the specific nutritional management of these patients is summarised. The maintenance of the structural and functional differentiation of the developing gut critically depends on adequate blood supply to meet the metabolic demand of the intestinal tissue. The second part of Chapter 2 presents a review of current literature concerning the regulation of intestinal perfusion.

To obtain insight into the functional maturation of the human intestinal epithelium and the influence of nutrition during the early postnatal period on this process, we conducted a randomised controlled trial in preterm neonates. In Chapter 3 the nutritive and barrier functions of the intestinal epithelium were investigated in preterm neonates of 25-32 weeks gestation. By means of a sugar absorptionpermeability test we determined passive (D-xylose) and active (3-O-methyl-Dglucose) carrier-mediated absorption of monosaccharides as well as epithelial permeability (lactulose/L-rhamnose ratio) at 1, 4, 7, and 14 days after birth. Neonates received total parenteral nutrition during the first postnatal week, and enteral nutrition was initiated thereafter.

With respect to the development of intestinal nutritive function our data showed that the ability of the intestinal epithelium to absorb monosaccharides by passive and active carrier-mediated transport is established by 25 weeks gestation and increases around the 30th week of gestation, independent of the presence of enteral nutrients. Regarding the development of intestinal barrier function we demonstrated that epithelial barrier function is attained by 25 weeks gestation. However, the intestinal permeability increased in the course of the first postnatal week, and restored upon the initiation of enteral feeding. This suggests that the 
preservation of the integrity of the epithelial barrier is dependent on the presence of luminal nutrients.

In Chapter 4 we evaluated the effects of early administration of small volumes of enteral nutrition on the maturation of the intestinal sugar absorptive capacity and barrier function in preterm neonates. To this end, neonates were randomly assigned to one of two feeding protocols. Neonates in the intervention group received $12 \mathrm{ml} /$ day of human milk or preterm formula in addition to parenteral nutrition during the first postnatal week. The control group only received parenteral nutrition during the first week after birth, and enteral feeding was initiated thereafter.

Neonates in the intervention group had higher rates of active carrier-mediated mono-saccharide absorption at the end of the second week as compared with the control group. Furthermore, intestinal permeability during the first postnatal week was lower in neonates receiving early enteral feeding. These data indicate that the administration of small amounts of enteral feeding shortly after birth is an effective nutritional intervention to accelerate the maturation of the sugar absorptive capacity of the intestine as well as to preserve the epithelial barrier function in very low birth weight neonates during the early postnatal period.

In the first part of Chapter 5 a novel foetal animal model is described, which was designed to study the microcirculation of the immature gut, as well as to investigate the maturation of vasomotor responses of the mesenteric resistance arteries and systemic haemodynamics in vivo during the second half of foetal development.

In the second part of Chapter 5 the response of the intestinal wall microcirculation to repetitive periods of asphyxia and reoxygenation was investigated at consecutive stages of foetal development. A temporary impairment of the oxygen supply followed by reoxygenation was not accompanied by an increase in leukocyte-vessel wall interactions, did not lead to leukocyte accumulation in the intestinal tissue, and did not induce histologic damage of the intestinal tissue. These findings contradict common theories regarding the role of ischaemia-reperfusion injury in the origin of necrotising enterocolitis. In contrast to the adult gut, leukocyte sequestration into the intestinal tissue after episodes of hypoxia-reoxygenation is not a major contributor to the initiation of injury in the immature gut.

In Chapter 6 the establishment of vasomotor regulation in the intestine during the second half of foetal development was investigated. To this end, we studied in vivo diameter changes in mesenteric resistance arteries, as well as changes in heart rate and blood pressure in response to pharmacologic stimuli and to acute hypoxia in chick foetuses between 0.6 and 0.8 of development.

With respect to the development of vasodilator control we demonstrated that a maximal vasodilator response to a variety of agents was already present at 0.6 gestation. This indicates that the vasodilator capacity of the mesenteric resistance arteries is already established at an early stage of foetal development. Regarding the maturation of vasoconstrictor control we showed that the adrenergic regulation of the intestinal circulation is initiated at approximately 0.6 gestation, is predo- 
minantly mediated by $\alpha$-adrenoceptors, and strongly increases towards the end of foetal gestation. Acute hypoxia was associated with a fall in heart rate and mean arterial pressure, and induced a transient constriction of the mesenteric arteries which was principally mediated by $\alpha$-adrenoceptors. The magnitude of the hypoxiaassociated constriction in the intestinal circulation considerably increased during the second half of foetal development, due to the enhancement of the $\alpha$-adrenergic constrictor capacity. The predominance of local vasodilator properties preserved gut blood flow despite a reduction in perfusion pressure.

In Chapter 7 we investigated the role of oxygen on the long-term establishment of local vasomotor and central haemodynamic control. To this end, we studied the effects of prolonged exposure to hypoxia on dilator and constrictor properties of mesenteric resistance arteries, as well as the structural and functional maturation of perivascular sympathetic nerves, in combination with measurements of blood pressure and heart rate in near-term chick foetuses. Chronic hypoxia was associated with functional sympathetic hyperinnervation, an increase in mesenteric arterial tone, but no change in blood pressure and heart rate at 0.9 of foetal development.

Together, the experimental data indicate that in the young foetus vasomotor control is established at the level of the individual organs, in order to preserve tissue oxygenation necessary for the structural and functional maturation of the developing organs. At the end of foetal development, central vasomotor control takes over, resulting in a hierarchical regulation of the circulation, which serves to maintain oxygen supply to those organs which are essential to the survival of the whole organism. Oxygen availability to the developing foetus appears to play a critical role in the long-term orchestration of the haemodynamic regulation in pace with the structural design of the cardiovascular system.

In Chapter 8 the outcomes of the clinical and experimental studies are discussed in light of the role of intestinal and cardiovascular maturation in the precocious adaptation to extrauterine life, as well as the implications for the pathogenesis and potential prevention of intestinal complications in preterm neonates. 


\section{SAMENVATTING}

Darmcomplicaties vormen in toenemende mate een belangrijk probleem bij de zorg voor te vroeg geboren baby's (premature neonaten). Een deel van deze kinderen heeft milde darmproblemen, zoals moeite met het verdragen van borst- of flesvoeding in de eerste weken na de geboorte. De meest ernstige darmaandoening bij premature neonaten is necrotiserende enterocolitis (NEC). Hierbij is er sprake van een ontsteking van een of meerdere gedeeltes van de dunne en/of dikke darm, die uiteindelijk leidt tot onherstelbare beschadiging van het darmweefsel. NEC komt bij 1-3 per 1000 pasgeborenen voor, en zelfs bij 6-10\% van de baby's geboren voor 32 weken zwangerschapsduur. Een op de drie getroffen neonaten overlijdt aan dit ziektebeeld. De behandeling bestaat in het merendeel van de gevallen uit chirurgische verwijdering van het afgestorven darmsegment. Ondanks een sterke verbetering van de klinische zorg voor te vroeg geborenen, en met name voor zeer vroeg geborenen, is NEC momenteel een van de belangrijkste doodsoorzaken bij deze patiênten. Ook bij de neonaten die de eerste moeilijke periode overleven, heeft dit ziektebeeld vaak ingrijpende complicaties op de langere termijn. De uitgebreide operatieve ingrepen op jonge leeftijd leiden niet alleen tot levenslange beperkingen van de darmfunctie, maar kunnen tevens ernstige ontwikkelingsstoornissen tot gevolg hebben.

Ondanks uitgebreid onderzoek is de oorzaak van darmcomplicaties bij te vroeg geborenen nog onbekend. Er wordt verondersteld dat drie factoren betrokken zijn bij het ontstaan van NEC: voeding, bacteriēn, en stoornissen in de bloeden zuurstofvoorziening van de darm. Echter, geen van deze factoren afzonderlijk kan het ontstaan van dit ziektebeeld volledig verklaren. Het is gebleken dat de kans op het ontwikkelen van darmcomplicaties nauw samenhangt met de mate van vroeggeboorte. Dit wijst erop dat immaturiteit of "onrijpheid" van de darm een centrale rol speelt in deze problematiek.

Voor de geboorte is de darm een relatief inactief orgaan, gevuld met steriel vruchtwater. Immers, de foetus krijgt zijn voedingsstoffen via de navelstreng direct in het bloed toegediend. Na de geboorte verandert deze situatie op twee fronten. Ten eerste is de baby afhankelijk van de darm voor de opname van voedingsstoffen uit melk. Bovendien komt de darm in contact met bacteriën, via de voeding, de moeder en de omgeving. Hierdoor worden twee belangrijke darmfuncties aangesproken, waarbij de darmcellen (het darmepitheel) een essentiële taak vervullen. Enerzijds is het darmepitheel verantwoordelijk voor de opname van voedingsstoffen (de nutritieve functie). Anderzijds biedt de nauwe interactie tussen intacte darmcellen bescherming tegen de invasie van schadelijke componenten uit de darm, zoals bacteriën en hun producten (de barrière functie).

Tijdens de foetale ontwikkeling wordt de darm op deze functies voorbereid. De structurele aanleg van het maagdarmkanaal vindt plaats tijdens de eerste helft van de zwangerschap en is reeds compleet rond de 20e week. De functionele ontwikkeling van de darm blijkt daarentegen een stuk langzamer te verlopen en vindt grotendeels plaats in de laatste paar maanden van de zwangerschap. Dit heeft belangrijke consequenties in geval van vroeggeboorte. Immers, in tegenstelling tot de normale zwangerschapsduur van 40 weken komt de te vroeg geborene in een 
eerder stadium van de ontwikkeling ter wereld. Als gevolg hiervan wordt een functioneel immature darm aan de omgeving blootgesteld en worden de nutritieve en barrière functies van de darm voortijdig vereist. Het is de vraag in hoeverre de darm hierop is voorbereid. Een onvoldoende adaptatie van de premature neonaat aan de overgang van het intrauteriene naar het extrauteriene milieu kan predisponeren tot intestinale complicaties.

In het eerste gedeelte van dit proefschrift werd bestudeerd in hoeverre de darm van te vroeg geboren baby's in staat is om suikers zoals glucose op te nemen (de absorptiecapaciteit) en tegelijkertijd de passage van mogelijk schadelijke componenten te voorkomen (de barrière functie). Immers, een van de belangrijkste energiebronnen voor de baby in borst- en flesvoeding is (melk)suiker. Bovendien werd onderzocht of het mogelijk is om de ontwikkeling van deze darmfuncties te stimuleren door toediening van kleine hoeveelheden borst- of flesvoeding kort na de geboorte, zogenaamde stimulatie-voeding.

Het onderzoek werd uitgevoerd bij 120 premature neonaten geboren tussen 25 en 32 weken zwangerschapsduur die waren opgenomen in de neonatale intensive care unit. Door middel van loting werd bepaald of de neonaten gedurende de eerste week na de geboorte uitsluitend voeding via een infuus kregen (late voeding groep) of infuusvoeding aangevuld met $12 \mathrm{ml} /$ dag borst- of flesvoeding toegediend via een maagsonde (vroege voeding groep). De absorptiecapaciteit en de doorlaatbaarheid, als maat voor de barrière functie, van de darm werden gemeten met behulp van een suikerabsorptietest op dag 1, 4, 7 en 14 na de geboorte.

De capaciteit van de darm om glucose op te nemen was laag bij neonaten jonger dan 28 weken, en steeg rond de $30 \mathrm{e}$ week. Vroege borst- of flesvoeding verhoogde de absorptiecapaciteit. De barrière functie van de darm was reeds ontwikkeld rond de 25 e week. Echter, bij de kinderen die alleen infuusvoeding kregen, nam de barrière functie van de darm tijdens de eerste week na de geboorte af. Deze afname werd voorkomen door vroege borst- of flesvoeding.

Deze resultaten tonen aan dat vroegtijdige toediening van kleine hoeveelheden borst- of flesvoeding een eenvoudige methode is om de maturatie van de nutritieve functie van de darm te stimuleren en de barrière functie van de darm te bevorderen bij premature neonaten.

In het tweede gedeelte van dit proefschrift werd de bloed- en zuurstofvoorziening van de zich ontwikkelende darm onderzocht. Immers, de ontwikkeling en instandhouding van de darm zijn afhankelijk van een adequate toevoer van bloed en zuurstof aan het darmweefsel. Stoornissen in de bloedvoorziening van de immature darm zijn mogelijk betrokken bij het ontstaan van darmcomplicaties bij premature neonaten. Er wordt verondersteld dat tijdens een kortdurend zuurstofgebrek van de baby, bijvoorbeeld gedurende de geboorte, de doorbloeding van de immature darm tijdelijk vermindert. Het tekort aan zuurstoftoevoer naar de darm en het optreden van een ontstekingsreactie na het herstel van de doorbloeding zouden kunnen leiden tot een onherstelbare beschadiging van het darmweefsel.

De doorbloeding van het darmvaatbed wordt hoofdzakelijk door twee factoren bepaald: de mate waarin de aanvoerende bloedvaten openstaan (de arteriële 
diameter) en de bloeddruk. Uit onderzoek bij volwassen dieren blijkt dat de arteriēle diameter door een groot aantal factoren wordt beinvloed. Deze factoren veroorzaken ofwel een toename in de diameter (dilatatie) ofwel een afname in de diameter (constrictie), en leiden daardoor respectievelijk tot een toe- of afname van de darmdoorbloeding. Deze factoren kunnen grofweg in twee groepen worden verdeeld. Factoren die lokaal in de darm worden geproduceerd, bijvoorbeeld in de vaatwand en in het darmweefsel, reguleren de arteriële diameter zodanig dat de bloedvoorziening wordt aangepast aan de behoefte van de darm zelf. Centrale factoren daarentegen coördineren de verdeling van het bloedvolume tussen de verschillende organen en zijn belangrijk bij de handhaving van de bloeddruk. Hiertoe behoren adrenaline, dat in het bloed vrijkomt vanuit de bijnier, en (sympathische) zenuwen rond de bloedvaten.

In tegenstelling tot de volwassen darm is het niet bekend hoe de doorbloeding van de immature darm wordt gereguleerd. De mogelijkheden om deze onderwerpen te bestuderen in de menselijke foetus en de te vroeg geborene zijn vanwege ethische en technische redenen zeer beperkt. Derhalve hebben wij een nieuw experimenteel diermodel opgezet, waarbij het vaatbed van de darmwand en de aanvoerende bloedvaten van de darm werden onderzocht bij kippenembryo's tijdens de tweede helft van de foetale ontwikkeling.

Ten eerste werd in dit model onderzocht of een kortdurende onderbreking van de zuurstoftoevoer aan het embryo aanleiding geeft tot een ontsteking en beschadiging van de darm. Als maat hiervoor werd de interactie tussen de witte bloedcellen (leukocyten) in het bloed en de vaatwand bepaald in de kleine bloedvaten (microcirculatie) van de darmwand. Deze experimenten tonen aan dat herhaalde periodes van kortdurend zuurstofgebrek geen toename in leukocyt-vaatwandinteracties en geen beschadiging van de darm veroorzaken. Echter, behalve als onderdeel van een ontstekingsproces, vormen leukocyt-vaatwandinteracties ook een belangrijke component van de aspecifieke afweer tegen bacteriën. De beperkte aanwezigheid van dergelijke interacties in de microcirculatie draagt derhalve bij tot een verminderde barrière functie van de immature darm.

Ten tweede werd in het kippenembryo model de regulatie van de doorbloeding van de darm bestudeerd op verschillende tijdstippen van de foetale ontwikkeling. Hiertoe werd met behulp van een microscoop de reactie van de aanvoerende bloedvaten van de darm gemeten op lokaal toegediende stoffen en op een kortdurende onderbreking van de zuurstoftoevoer aan het embryo. Deze experimenten tonen aan dat in het jonge embryo het vermogen tot constrictie van de bloedvaten in de darm slechts beperkt is, terwijl het vermogen tot dilatatie in dit stadium wel al volledig is ontwikkeld. Naarmate de ontwikkeling van het embryo vordert, neemt het vermogen tot constrictie toe. Bovendien blijkt dat de doorbloeding van de darm in eerste instantie wordt gereguleerd door lokale factoren en dat centrale regulatie pas tegen het einde van de ontwikkeling gaat overheersen. Deze balansverschuiving heeft belangrijke consequenties wanneer de zuurstoftoevoer aan het embryo tijdelijk wordt verminderd. Het jonge embryo kan onder dergelijke omstandigheden wel de bloedvoorziening van de darm, maar niet de bloeddruk handhaven. Dit kan leiden tot verminderde doorbloeding van de organen die vitaal zijn voor de overleving van het individu, zoals het hart en de hersenen, en derhalve 
tot de dood. Daarentegen is het oude embryo goed in staat om zijn bloeddruk in stand te houden, ofschoon dit ten koste gaat van de darmdoorbloeding.

Deze experimentele gegevens bieden nieuw inzicht in de rol van stoornissen in de bloed- en zuurstofvoorziening in het ontstaan van darmcomplicaties bij te vroeg geborenen. $W_{i j}$ denken nu dat darmschade bij premature neonaten (vergelijk het jonge embryo) niet primair wordt veroorzaakt door een lokale stoornis in de bloed- en zuurstofvoorziening van de darm, maar dat darmcomplicaties bij deze kinderen een lokaal gevolg zijn van een algeheel onvermogen tot adequate regulatie van de bloeddruk.

Met het oog op het groeiende aantal zeer vroeg geborenen en de toenemende overlevingskansen van deze kinderen zullen darmcomplicaties in de toekomst naar verwachting een steeds groter probleem worden op de neonatale intensive care unit. De ernst van deze aandoeningen onderstreept de noodzaak tot fundamenteel wetenschappelijk en klinisch onderzoek naar de verdere ontwikkeling van preventieve maatregelen en nieuwe behandelingsmethoden. 


\section{Dankwoord}

Graag maak ik van deze gelegenheid gebruik om iedereen te bedanken die aan de totstandkoming van dit proefschrift heeft bijgedragen.

Een bijzonder woord van dank gaat uit naar:

De begeleiders

Prof. dr. G. Ramsay

Prof. dr. D.W. Slaaf

Dr. F.A.C. le Noble

Prof. dr. E. Heineman

Prof. dr. W.A. Buurman

Prof. dr. C.E. Blanco

Dr. G. ter Riet

De vakgroepen

Algemene Chirurgie

Kindergeneeskunde/Neonatologie

m.n. alle verpleegkundigen van de NICU, drs. B. Panis en Mw. A. Gerver

Fysiologie

m.n. het laboratorium voor Microcirculatie

Anatomie/Embryologie

Farmacologie

Klinische Chemie

en alle ondersteunende diensten

De compagnons

Martijn Broeders

Xander Wehrens

Esther Lutgens

Tim Leiner

Roland Beuk

Marc Daemen

Bart Stubenitsky

François van Dielen

Misha Luyer

Jolanda van Golde

Ruben Dammers

Tryfon Vainas

En natuurlijk mama, papa en Wim. 


\section{CURRICULUM VITAE}

Name:

Date of birth:

Place of birth:
Ellen Valérie Rouwet

January 30,1973

Margraten, the Netherlands

\section{Education}

1985-1991 Gymnasium-B, Jeanne d'Arc College Maastricht, the Netherlands

1991-1992 Propaedeuse Medicine, University of Maastricht, the Netherlands

1992-1995 Doctoral phase Medicine, University of Maastricht, the Netherlands (cum laude)

1995-2001 Internships Medicine, University of Maastricht, the Netherlands

January 2002 Start residency in General Surgery, University Hospital Maastricht, the Netherlands

Research

1993-1995 Student researcher, department of General Surgery, University Hospital Maastricht, the Netherlands

1996 Research fellow, department of Physiology, University of Maastricht, the Netherlands

1997-2002 PhD student, department of General Surgery, University Hospital Maastricht, and department of Physiology, Cardiovascular Research Institute Maastricht, the Netherlands under the guidance of Prof. dr. G. Ramsay, Prof. dr. D.W. Slaaf, Dr. F.A.C. le Noble

\section{Awards}

Student Research Prize 1995, Faculty of Medicine, University of Maastricht, the Netherlands

Dutch Hippokrates Award 1995, University of Leiden, the Netherlands

Young Investigator Award, World Congress of Pediatric Gastroenterology, Hepatology and Nutrition, August 2000, Boston, USA

Prize for Best Presentation, 13 th SEOHS symposium for experimental surgical research, November 2000 , Utrecht, the Netherlands 
Kurvers H., Stassen F., Jacobs M., Daemen M., Rouwet E., Van den Wildenberg F., Kitslaar P., Slaaf D., Reneman R., and De Mey J. Microcirculatory abnormalities in reflex sympathetic dystrophy and an animal model of neuropathic pain; hypersensitivity to catecholamines consequent to autonomic denervation? NVBP-pijnbulletin 1994; 2: 2-4.

Spincemaille G., Barendse G., Rouwet E., Kurvers H., Adang E., Van Kleef M., Dingemans W., and Van den Wildenberg F. Spinal cord stimulation and reflex sympathetic dystrophy; review of the literature and proposal for a protocol. The Pain Clinic 1995; 8: 155-160.

Daemen M., Kurvers H., Kitslaar P., Van den Wildenberg F., Beuk R., Rouwet E., Jacobs M., and Reneman R. Microcirculatoire aspecten van een neuropathisch pijnsyndroom: de rol van het sympathische zenuwstelsel. Nederlands Tijdschrift voor Vaatdiagnostiek 1995; 8: 9-13.

Rouwet E., Spincemaille G., Barendse G., Van Kleef M., Adang E., Kurvers H., Daemen M., and Van den Wildenberg F. Spinal cord stimulation in de behandeling van reflex sympathische dystrofie. NVBP-pijnbulletin 1996; 16: 8-11.

Tromp S., Slaaf D., Van Breda E., Tangelder G.J., Reneman R., Rouwet E., and Oude Egbrink M. Are mast cells involved in regulation of leukocyte rolling in mesenteric venules of anesthetized rabbits? In: Proceedings of the Sixth World Congress for Microcirculation, edited by K. Messmer and W.M. Kübler. Monduzzi Editore, Bologna, Italia. 1996: p. 649-653.

Kurvers H., Tangelder G.J., De Mey J., Slaaf D., Van den Wildenberg F., Kitslaar P., Reneman R., Rouwet E., and Jacobs M. Skin blood flow disturbances in the contralateral limb in a peripheral mononeuropathy in the rat. Neuroscience 1996; 74 : 935-943.

Kurvers H., Tangelder G.J., De Mey J., Reneman R., Slaaf D., Rouwet E., Van den Wildenberg F., Kitslaar P., and Jacobs M. The influence of partial nerve injury in the rat on efferent function of sympathetic and antidromically acting sensory nerve fibers. J Trauma 1996; 41: 981-988.

Rouwet E., Beuk R., Heineman E., Slaaf D., and Oude Egbrink M. Effect of repetitive asphyxia on leukocyte-vessel wall interactions in the developing intestine. J Pediatr Sung 2000; 35: 49-55.

(Chapter 5, this thesis) 
Rouwet E., De Mey J., Slaaf D., Heineman E., Ramsay G., and Le Noble F. Development of vasomotor responses in fetal mesenteric arteries. Am J Physiol Heart Cinc Physiol 2000; 279: H1097-H1105.

(Chapter 6, this thesis)

Wehrens X., Rouwet E., Oude Egbrink M., Slaaf D., and Ramsay G. Effects of experimental lower limb ischaemia-reperfusion injury on the mesenteric micro circulation. Br J Surg 2002 ; 89 : 185-191.

Rouwet E., Heineman E., Ter Riet G., Ramsay G., Buurman W., and Blanco C. Intestinal permeability and carrier-mediated monosaccharide absorption in preterm neonates during the early postnatal period. Pediatr Res 2002; 51: 64-70.

(Chapter 3, this thesis)

Rouwet E., Blanco C., Ter Riet G., Ramsay G., Buurman W., and Heineman E. Early minimal enteral feeding in very low birth weight neonates: an effective strategy to improve mucosal functions of the immature gut. Submitted.

(Chapter 4 , this thesis)

Rouwet E., Tintu A., Schellings M., Van Bilsen M., Lutgens E., Hofstra L., Slaaf D., Ramsay G., and Le Noble F. Embryonic hypoxia induces aortic hypertrophic growth, left ventricular dysfunction, and sympathetic hyperinnervation of peripheral arteries in the avian fetus. Submitted.

(Chapter 7, this thesis) 


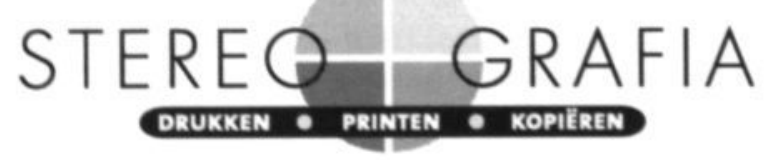





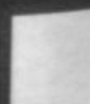

